\title{
A screening strategy to identify novel immunomodulators
}

\author{
By \\ Vimal Patel
}

A thesis submitted to the Victoria University of Wellington in fulfilment of the requirements for the degree of Doctor of Philosophy

Victoria University of Wellington 


\section{Abstract}

Understanding the immunomodulatory activities of compounds is important to identify the unintended adverse immunomodulatory effects of therapeutic compounds in development and to select novel compounds that may provide benefit for those diagnosed with immunemediated disorders. In both these cases, it is desirable to identify compounds with immunomodulatory activity early in the drug discovery process in a medium-throughput format. A screening strategy has been designed to fulfil these needs.

The first step in designing the strategy was to select informative assays and optimise individual assays to suit medium-throughput drug discovery. These individual assays investigated effects on a broad range of functions associated with innate and adaptive immune cells including macrophages (activation, cytokine production, phagocytosis and motility), helper $\mathrm{T}$ cells (activation and cytokine production), cytotoxic $\mathrm{T}$ cells (degranulation and cytokine production), and B cells (antibody production and cytokine production). Cost effectiveness and ease-of-use were important considerations during assay design and optimisation.

Using a compound set comprised of positive controls (i.e. compounds known to alter specific immune functions), a data set was generated to guide the strategy design. Assays were ordered to efficiently use resources and reduce the generation of less informative data. Additionally, using data collected from this compound set, strategies to assess and identify immunomodulatory activity were built and analysed. A second set of compounds was used to validate the screening strategy, and this screen highlighted new and novel activities for these known compounds that suggests they possess additional immunomodulatory effects.

Once validated, several novel compounds were run through the screen, including a traditional Samoan medicine, a heparan sulfate mimetic, and a novel anti-cancer agent; unique immunomodulatory activities were discovered. Finally, a hierarchical cluster analysis was used to cluster compounds sharing similar activity profiles and suggested the potential to develop further statistical methods to provide insight into compound characterisation. Together, this research has developed and validated a novel, medium throughput drug discovery system that can facilitate the identification of the immunomodulatory activities of compounds in the drug discovery environment. 


\section{Acknowledgements}

All that I've achieved is due to the people around me, and for that I owe many thanks.

First, to my supervisor Professor Anne La Flamme, thank you for guiding and supporting me over the past few years. You've not only taught me much about the complexities of immunology but also about how to encourage people to do their best. To my secondary supervisor, A/Professor Paul Teesdale-Spittle, thank you for sharing what you know which is impressively expansive. Thank you for your compassion and your delightful sense of humour.

To Ethan, you're a great friend. Thank you for the laughs, the beers, and the creativity you shared. Sven, your cheeky sense of humour made these last few years a lot of fun. You've taught me so much, not only about the mysterious world of MALDI but also how to be a better teacher. For your patience and humour and friendship (and coffee dates), I am ever grateful. Matt Rowe, thank you for long yarns late into the night during long experiments and for bouncing around ideas about new experiments and techniques to try out. I enjoy seeing you achieve great things. Dini, where would I be without you? You've helped me with so much and I hope one day I can repay the favour. Alvey, thanks for being your delightful self, for the uplifting puppy pictures, and for making me lunch on my long days in the lab. To my chemists, Kristen, Chriselle and Thomas, thank you for your friendship and kindness, and the chance to learn new things from you.

To Seeseei, Tim, Jono, and Callum: I enjoyed collaborating with you throughout my project. To the academics: John, Joanna, Melanie, Lisa, and Andrew, I appreciate your sharing of knowledge and advice, it was a pleasure working with you.

To my current lab group: Katharina, Carl, Nikki, Lisa D, Ali and Daniela, you've been such a great bunch to work with. To lab members of the past, especially Pirooz, Lisa J, and Yanyan, thanks for sharing this journey with me. To the AM family (you too, ChemGen), I can't think of a better group of people to have spent this time with.

Thank you all. 


\section{Table of Contents}

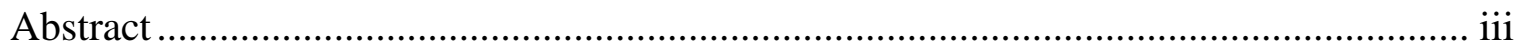

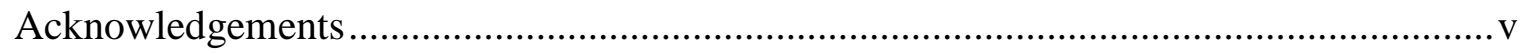

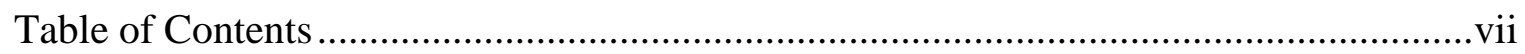

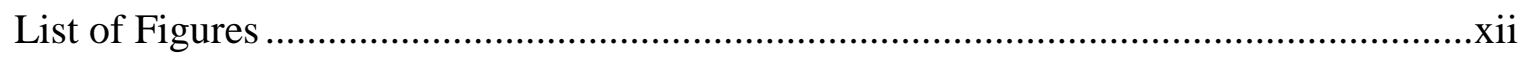

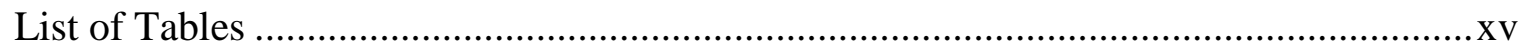

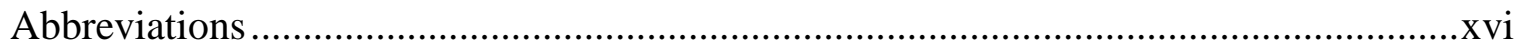

Chapter 1: Introduction ..................................................................................................................... 19

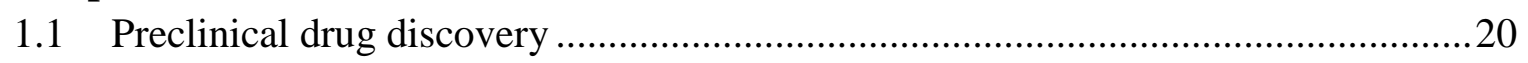

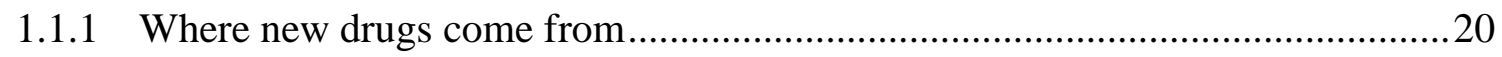

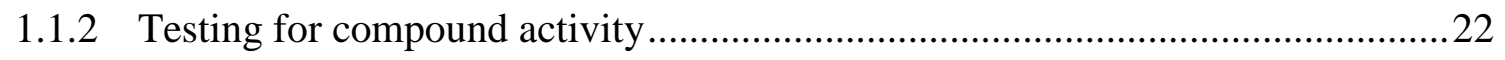

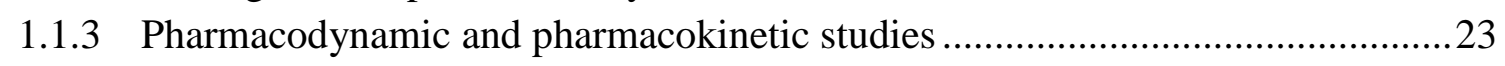

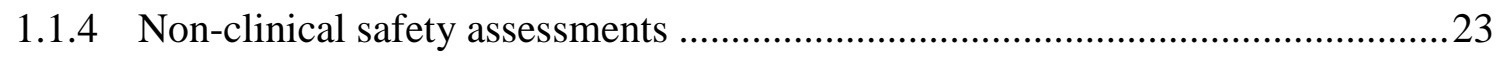

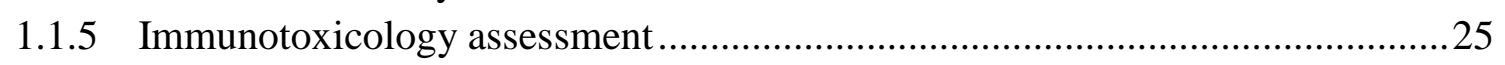

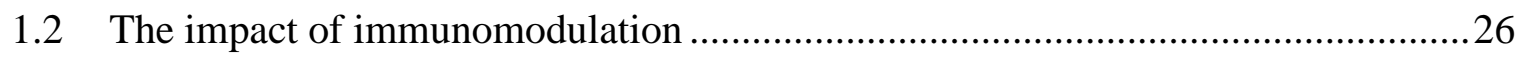

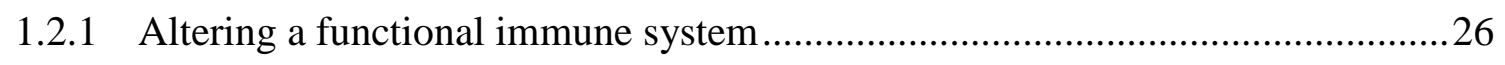

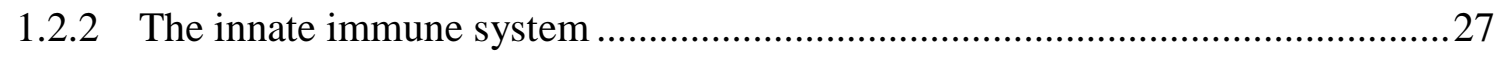

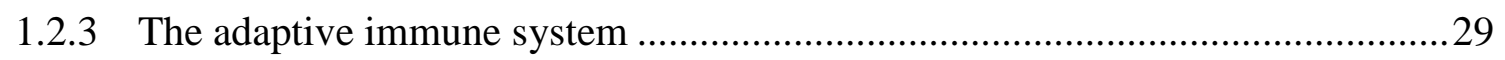

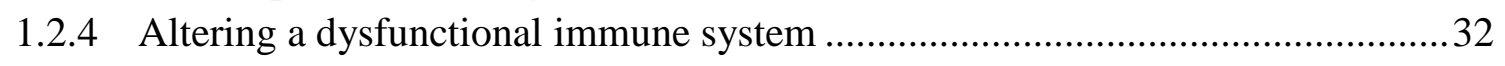

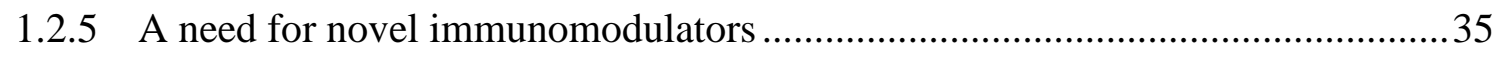

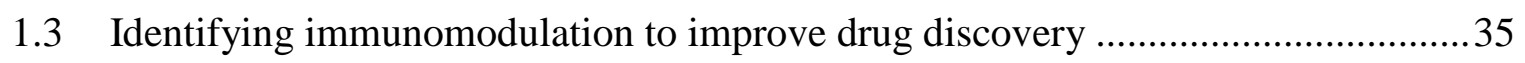

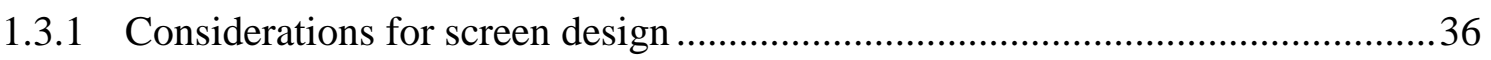

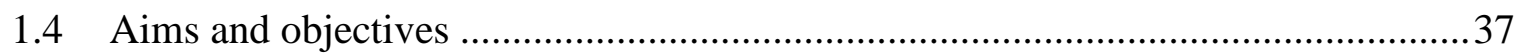

Chapter 2: Methods ........................................................................................................................ 39

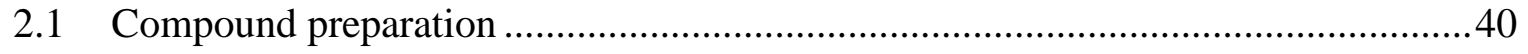

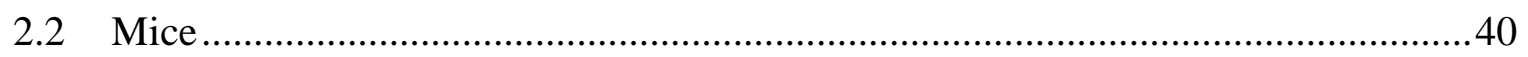

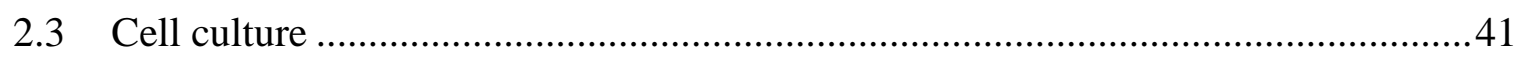

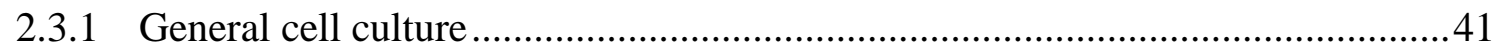

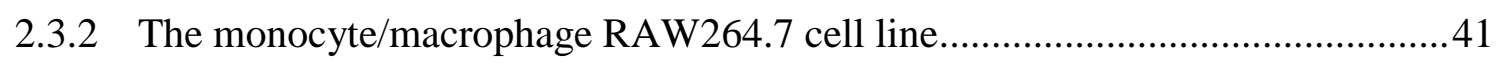

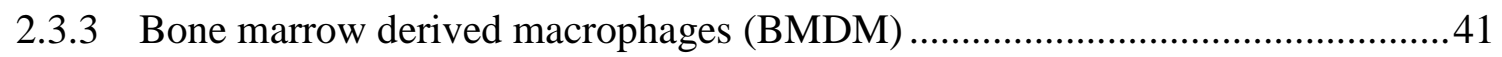

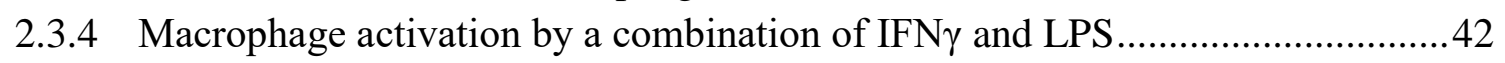

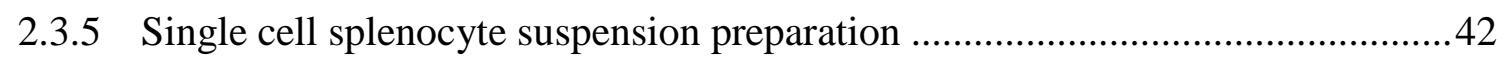

2.3.6 Helper T cell activation: ConA-stimulated splenocytes ......................................43

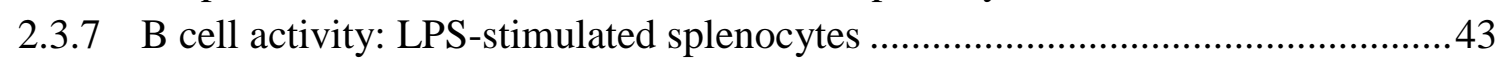

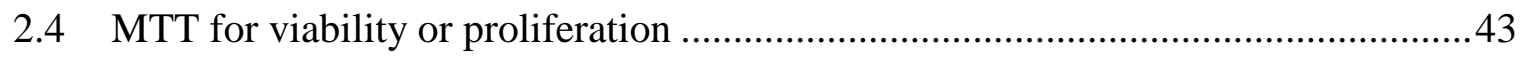

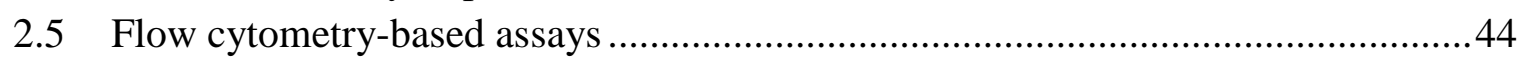

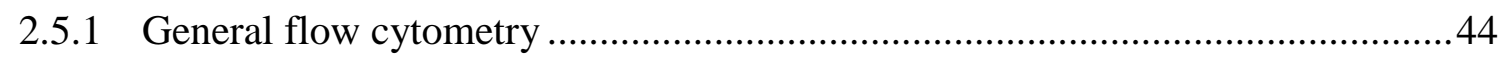

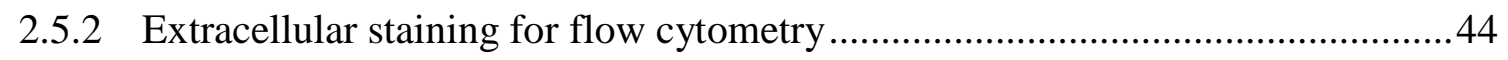

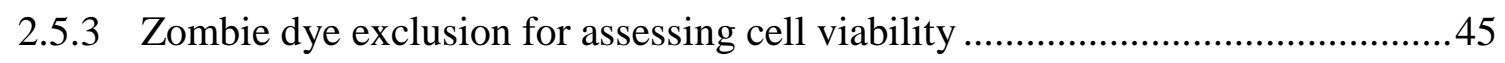

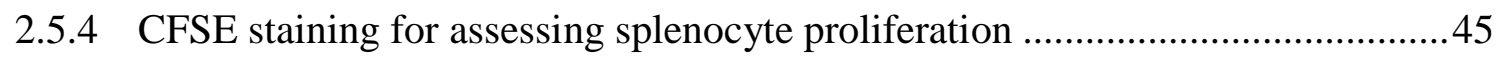

2.5.5 Assessing macrophage phagocytosis using pHrodo bioparticles .........................45

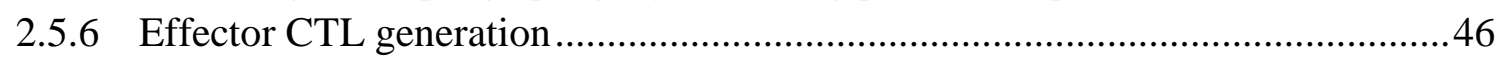




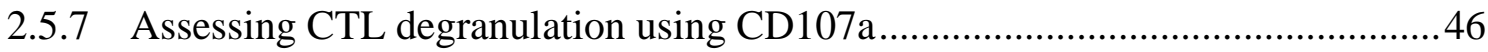

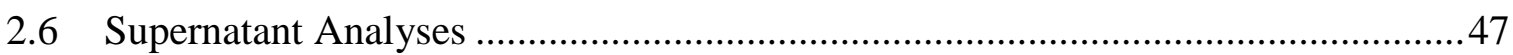

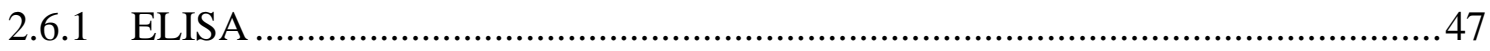

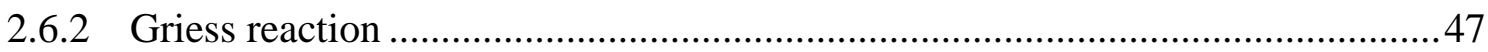

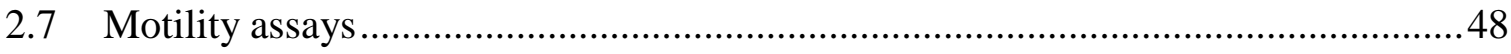

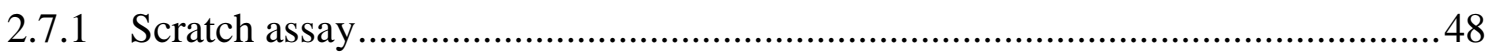

2.7.2 ORIS wound closure assay for motility ......................................................... 48

Chapter 3: Optimising assays to detect immunomodulation ..................................... 49

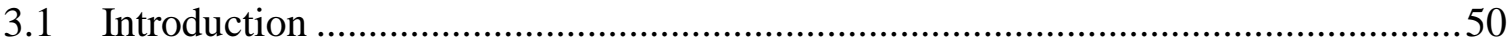

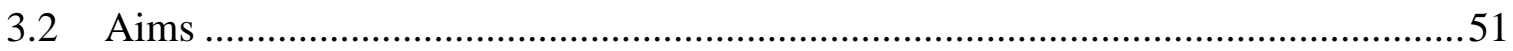

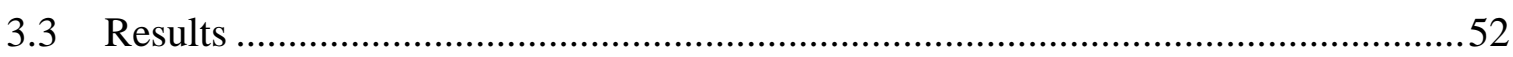

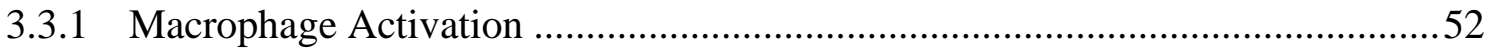

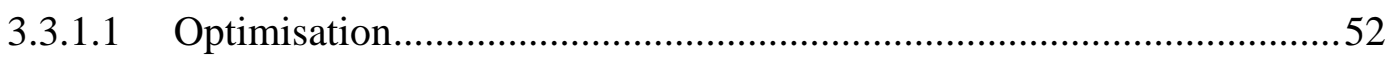

3.3.1.2 Altering macrophage activation with Risperidone ..............................56

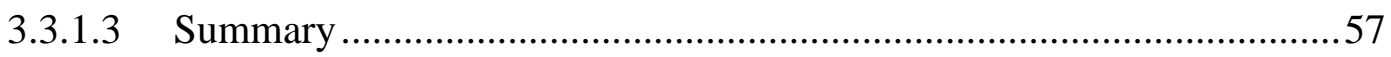

3.3.2 Assessing phagocytosis with pHrodo bioparticles ........................................... 58

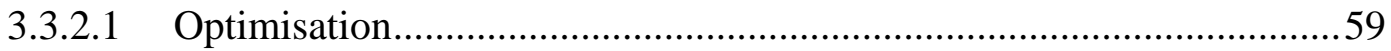

3.3.2.2 Inhibiting phagocytosis with Latrunculin A ........................................60

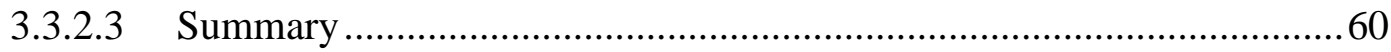

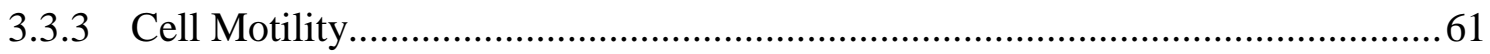

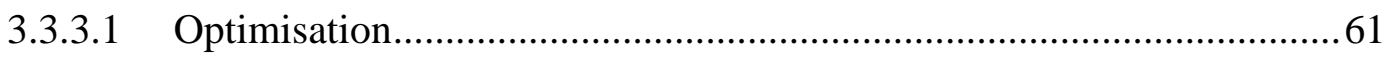

3.3.3.2 Latrunculin A inhibits macrophage motility........................................64

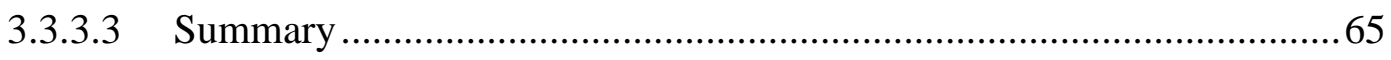

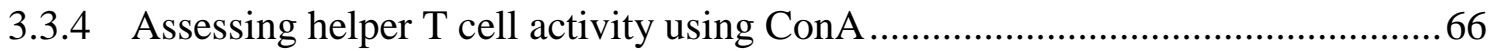

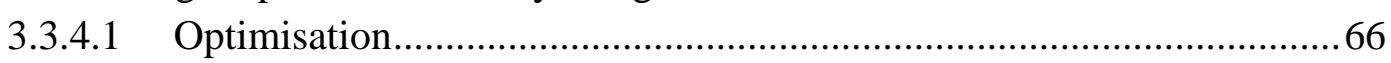

3.3.4.2 Glatiramer acetate alters Th cytokine production .................................. 70

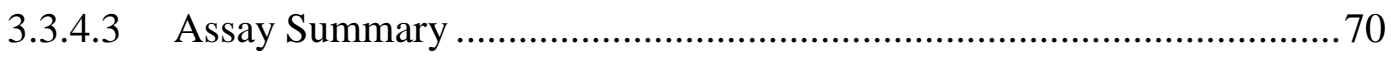

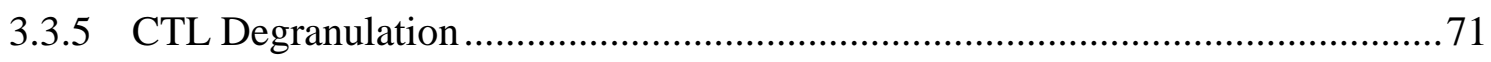

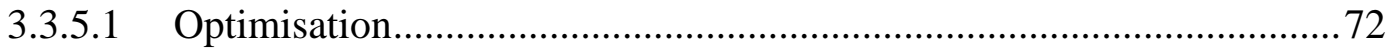

3.3.5.2 Colchicine inhibits CTL degranulation and IFN $\gamma$ production ............... 78

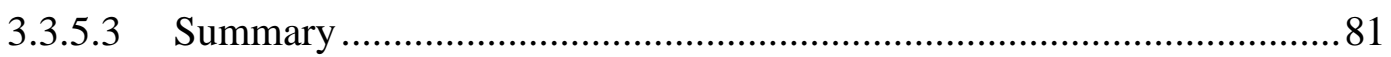

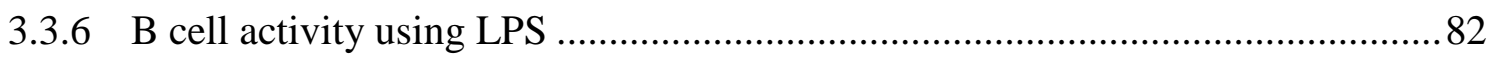

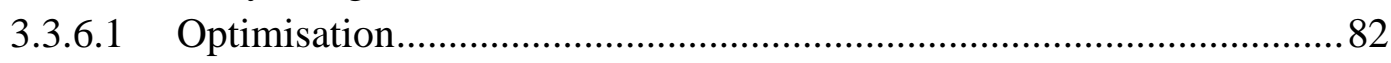

3.3.6.2 Inhibiting LPS-stimulated B cell activity with cycloheximide..............85

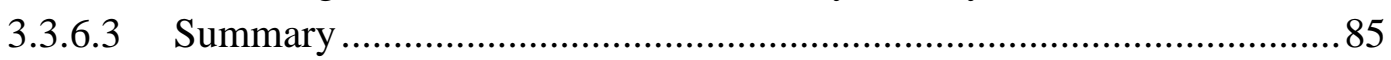

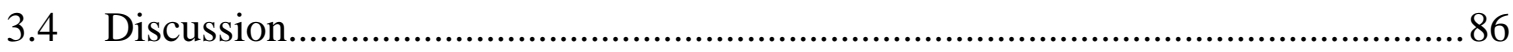

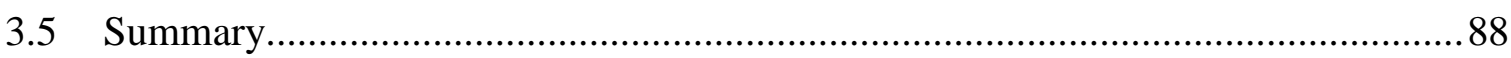


Chapter 4: Building the screening strategy .......................................................................89

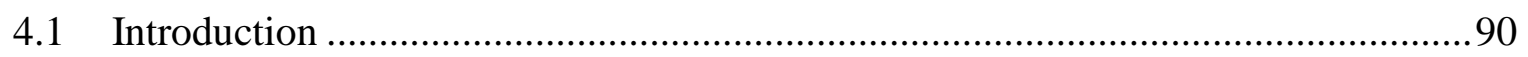

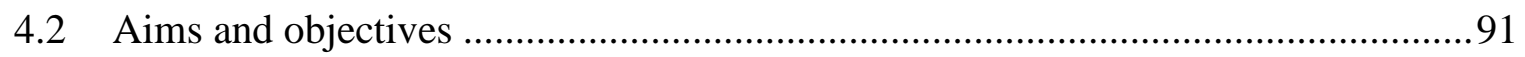

4.3 Results ..................................................................................................92

4.3.1 The Screen-building compound set ..................................................................92

4.3.2 Screen-building set compounds: effects on macrophage activation.....................99

4.3.3 Screen-building set compounds: effects on macrophage viability ........................97

4.3.4 Screen-building set compounds: effects on macrophage phagocytosis..............100

4.3.5 Screen-building set compounds: effects on macrophage motility ……...............103

4.3.6 Screen-building set compounds: effects on helper T cell activation ...................105

4.3.7 Screen-building set compounds: effects on splenocyte viability..........................107

4.3.8 Screen-building set compounds: effects on CTL Degranulation.........................110

4.3.9 Screen-building set compounds: effects on B cell activity..................................112

4.3.10 Activity summary of the compounds in the screen-building set ........................114

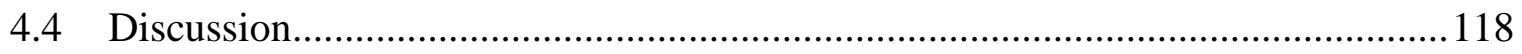

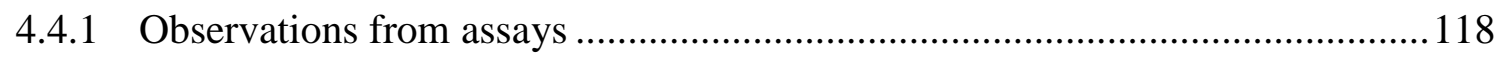

4.4.2 Considerations for the screening strategy build.................................................120

4.4.3 Designing the screening strategy …………………........................................123

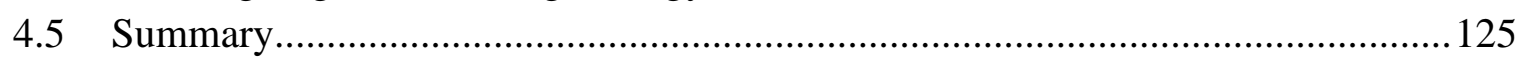

Chapter 5: Evaluating the performance of the screening strategy ..............................127

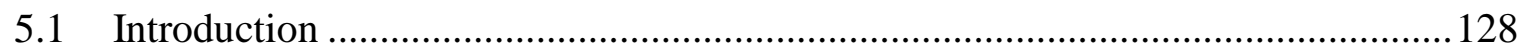

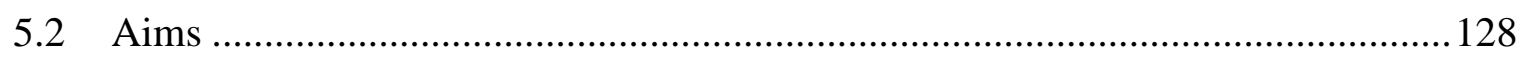

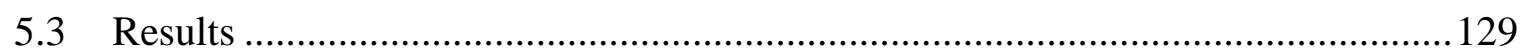

5.3.1 A modification to the concentration selection stage of screening ......................130

5.3.2 The activity profiles of compounds in the validation set.....................................137

5.3.3 Screening data can adequately identify immunomodulatory activity.................146

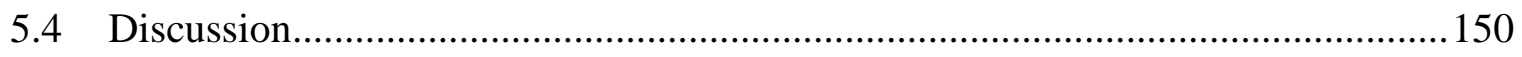

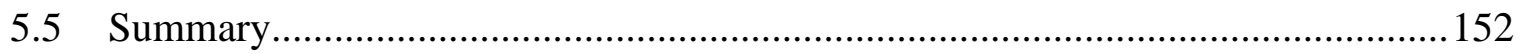

Chapter 6: Novel immunomodulators .................................................................153

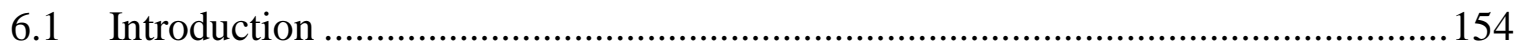

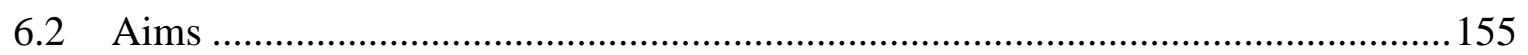

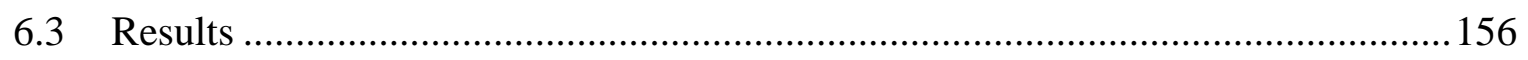

6.3.1 Immunomodulation by Psychotria insularum, a medicinal plant .......................156

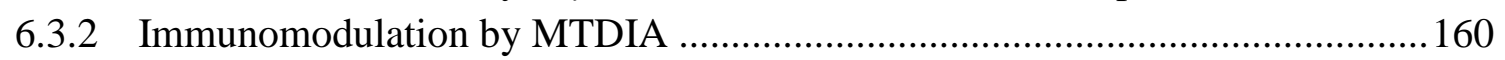

6.3.3 Immunomodulation by HS 16 - 38, a Heparan sulfate mimetic ..........................162

6.3.4 Immunomodulation by neurotransmitter receptor-targeting compounds ...........163

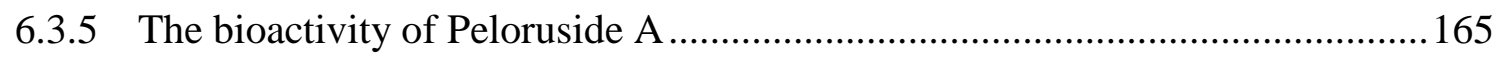

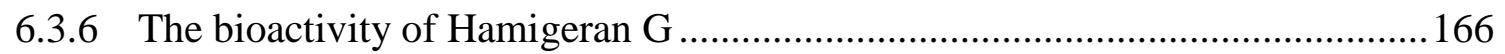

6.3.7 A cluster analysis for novel compound activity overview...................................167

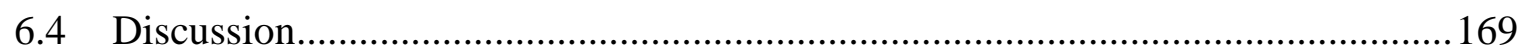

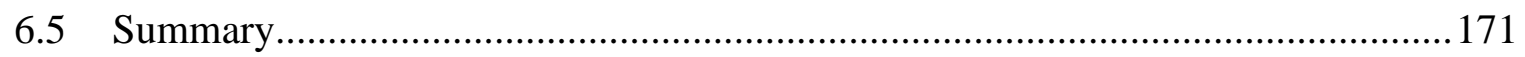


Chapter 7: General Discussion ..........................................................................................173

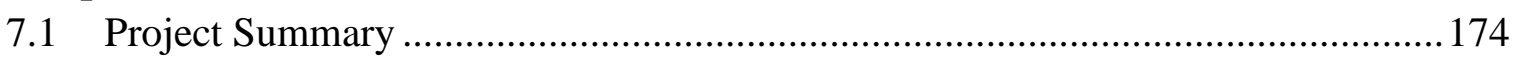

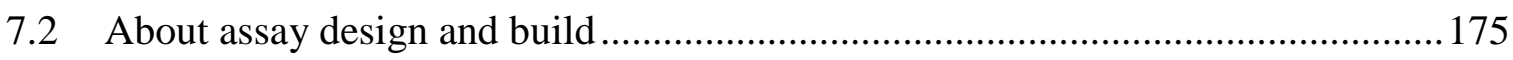

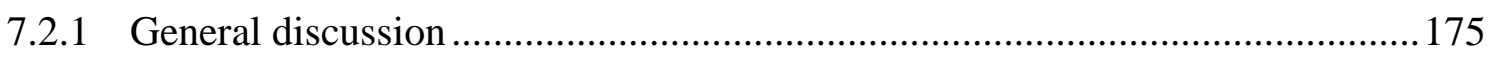

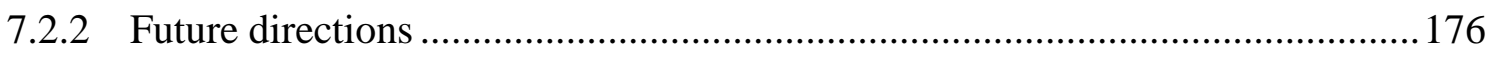

7.3 About assessing immunomodulatory activities .....................................................180

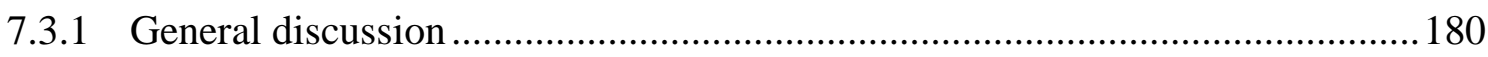

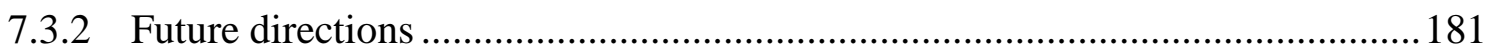

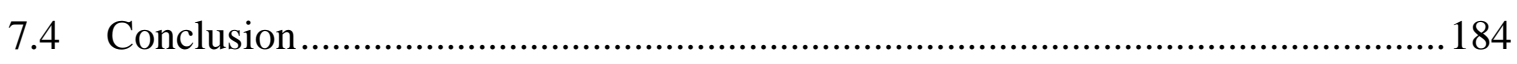

References ........................................................................................................................... 185

Appendices...............................................................................................................195

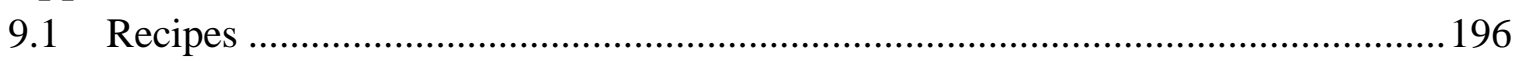

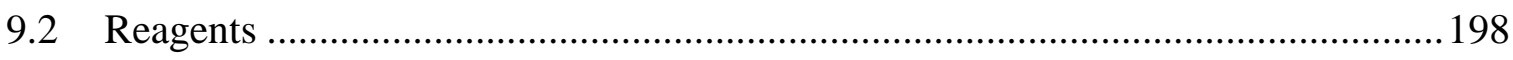

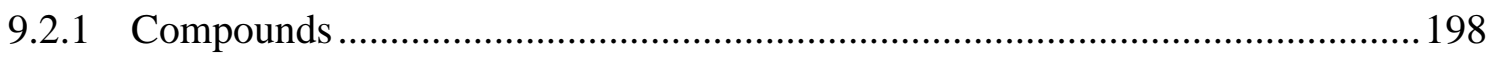

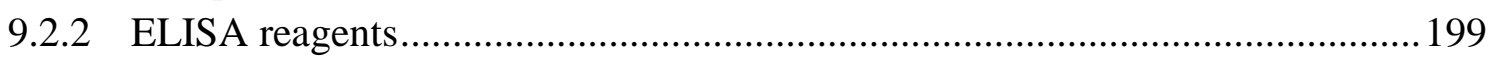

9.2.3 Flow cytometry antibodies and dyes ..............................................................20

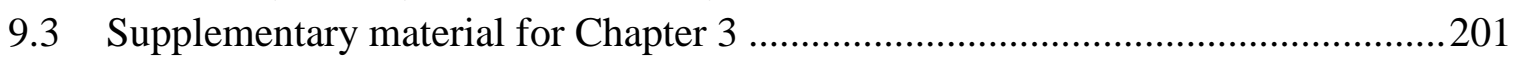

9.3.1 Gating strategy: Phagocytosis by RAW 264.7 cells ...........................................201

9.3.2 Gating strategy: B and T cell proliferation with CFSE ………..........................202

9.3.3 Gating strategy: to identify subpopulations during eCTL generation ................203

9.3.4 Gating strategy: Identifying eCTL by FCS and SSC.........................................204

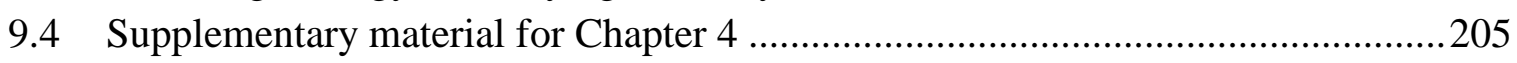

9.4.1 Immunomodulation by screen-building set compounds in BMDM ..................205

9.4.2 Assessing macrophage viability with MTT and Zombie dyes ...........................207

9.4.3 The effects of compounds on B cell viability ………………….........................208

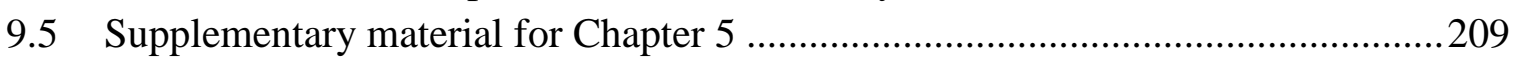

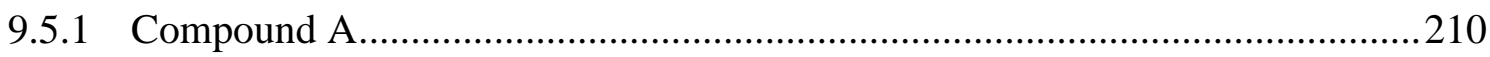

9.5.1.1 Concentration selection for compound A ...........................................210

9.5.1.2 Screening data for Compound A.......................................................211

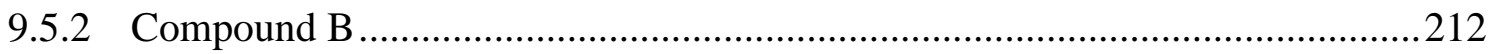

9.5.2.1 Concentration selection for compound B ..........................................212

9.5.2.2 Screening data for Compound B..........................................................213

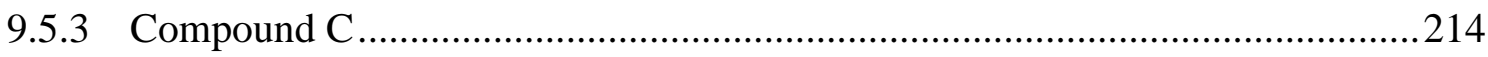

9.5.3.1 Concentration selection for compound C ...........................................214

9.5.3.2 Screening data for Compound C..........................................................2.

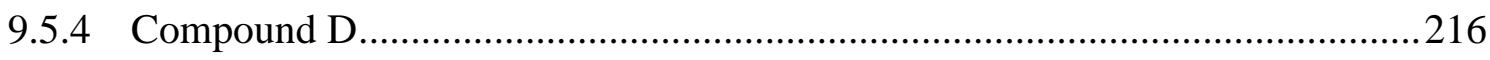

9.5.4.1 Concentration selection for compound D ............................................216

9.5.4.2 Screening data for Compound D..........................................................217

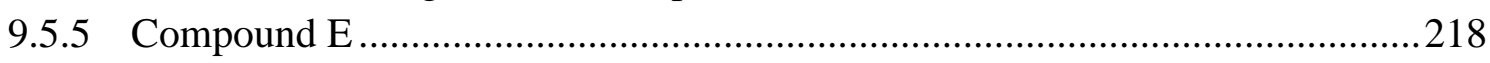

9.5.5.1 Concentration selection for compound E...........................................2218

9.5.5.2 Screening data for Compound E ...........................................................219

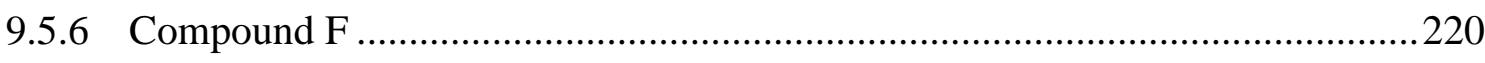

9.5.6.1 Concentration selection for compound F ............................................220 
9.5.6.2 Screening data for Compound F ....................................................221

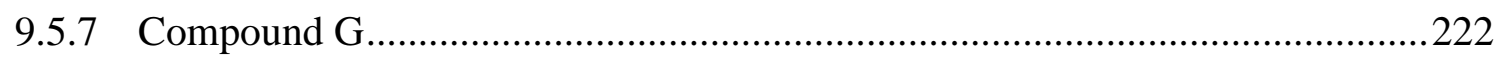

9.5.7.1 Concentration selection for compound G ......................................222

9.5.7.2 Screening data for Compound G....................................................223

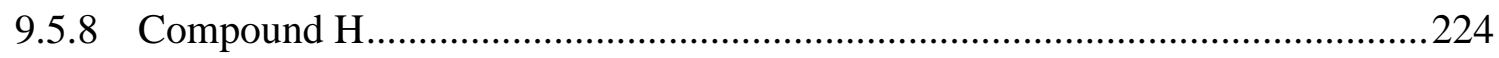

9.5.8.1 Concentration selection for compound $\mathrm{H}$.........................................224

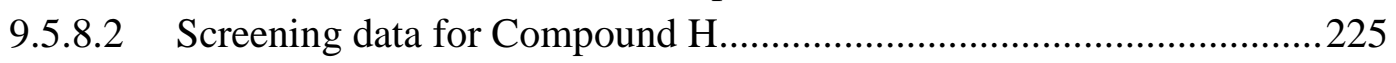

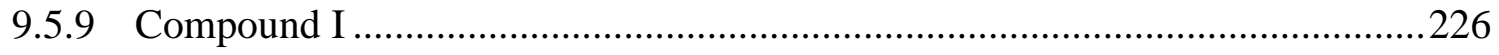

9.5.9.1 Concentration selection for compound I........................................226

9.5.9.2 Screening data for Compound I ...................................................22

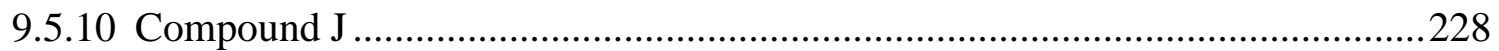

9.5.10.1 Concentration selection for compound J....................................228

9.5.10.2 Screening data for Compound J ..................................................229

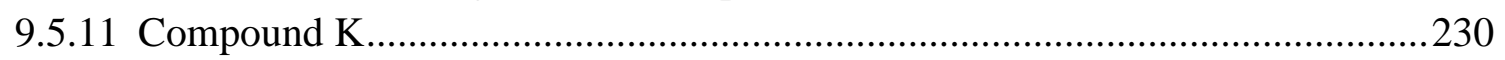

9.5.11.1 Concentration selection for compound K ....................................230

9.5.11.2 Screening data for Compound K.................................................2231

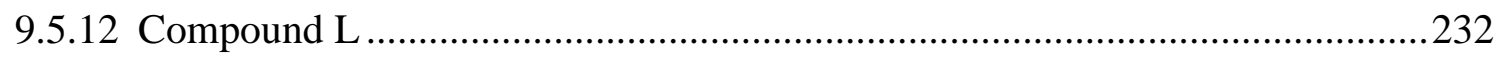

9.5.12.1 Concentration selection for compound L ......................................232

9.5.12.2 Screening data for Compound L ...................................................233

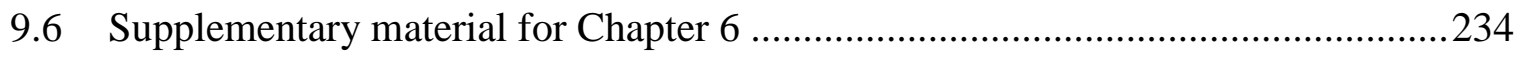

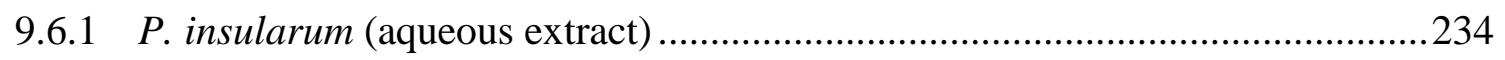

9.6.1.1 Screening data for P. insularum (aqueous extract) ............................2234

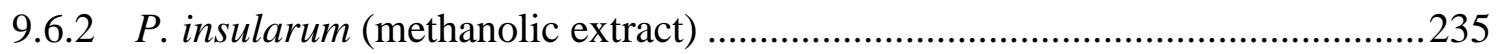

9.6.2.1 Concentration selection for P. insularum (methanolic extract) ............235

9.6.2.2 Screening data for P. insularum (methanolic extract).........................236

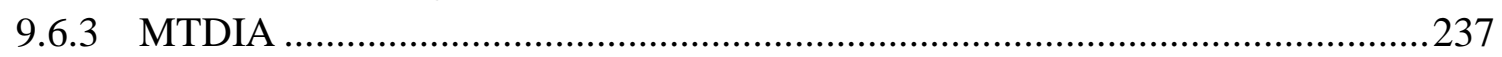

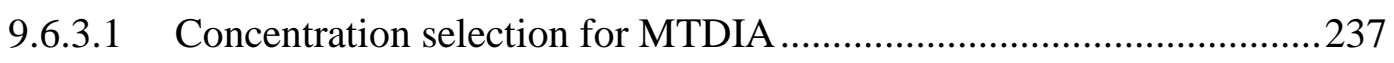

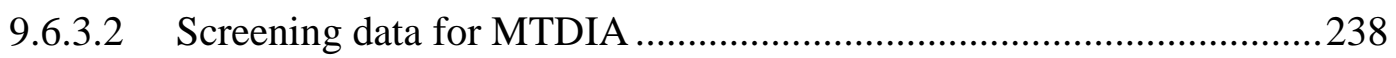

9.6.3.3 RAW cell viability with MTA and MTDIA treatment ........................2239

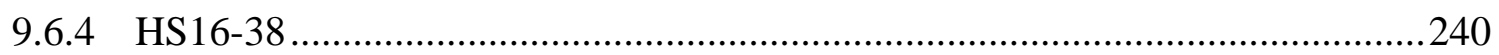

9.6.4.1 Concentration selection for compound HS16-38................................240

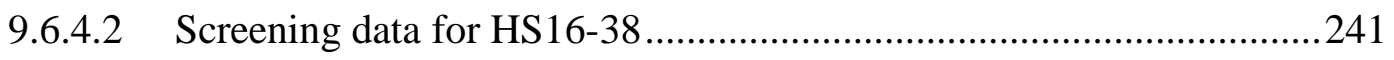

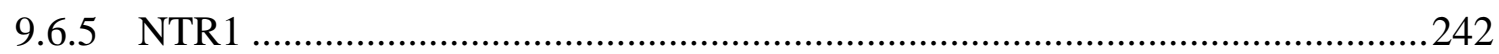

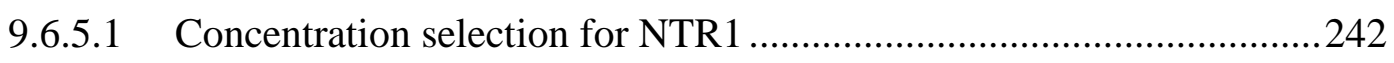

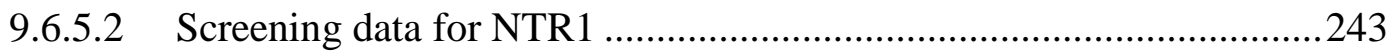

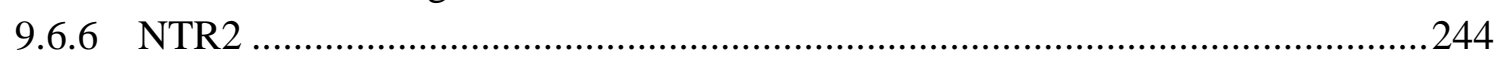

9.6.6.1 Concentration selection for NTR2 …...........................................244

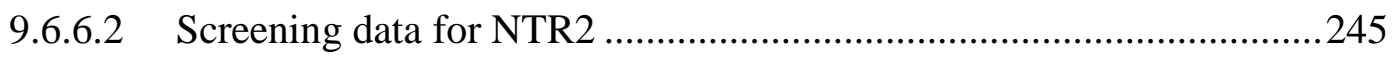

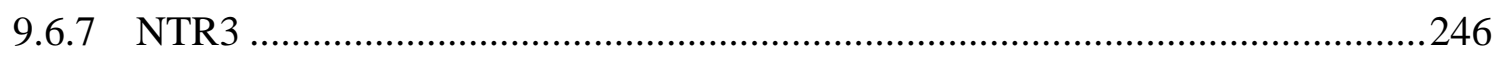

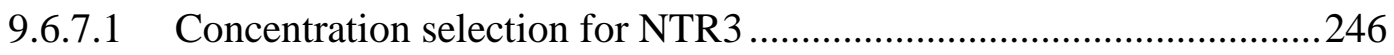

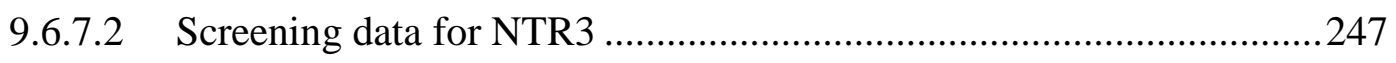




\section{List of Figures}

Figure 3.1. Priming macrophages with $20 \mathrm{U} / \mathrm{mL} \mathrm{IFN} \gamma$, and stimulating with $200 \mathrm{ng} / \mathrm{mL}$ LPS, induces

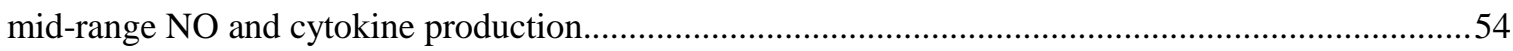

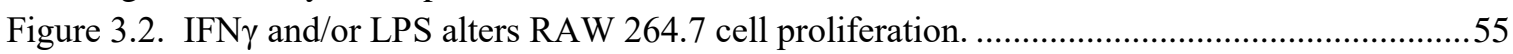

Figure 3.3. Risperidone alters cytokine production in activated RAW 264.7 cells. ..............................56

Figure 3.4. Risperidone shifts activated BMDM cytokine production to resemble an M2 phenotype. 57

Figure 3.5. $4 \mathrm{~h}$ pHrodo incubation allows for TNF $\alpha$ measurement as well as phagocytosis assessment.

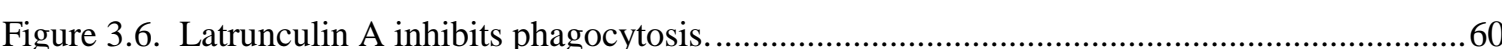

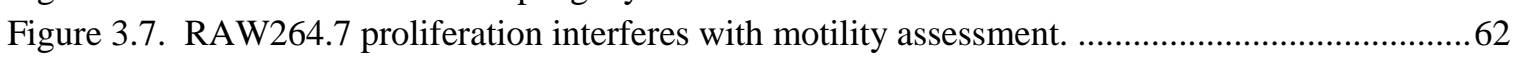

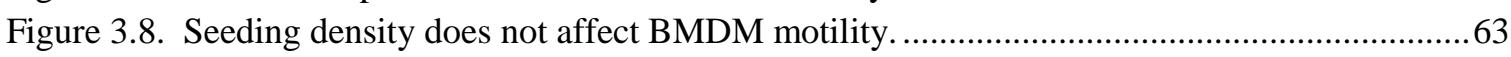

Figure 3.9. Inhibition of motility using LatA is measurable using the ORIS assay................................65

Figure 3.10. ConA stimulation of splenocytes induces helper $\mathrm{T}$ cell differentiation into four Th subsets.

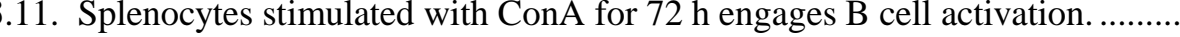

Figure 3.12. T cells proliferate in response to ConA stimulation. .......................................................69

Figure 3.13. $1 \mu \mathrm{g} / \mathrm{mL}$ ConA activation of $\mathrm{T}$ cells is modulated by Glatiramer acetate. ......................... 70

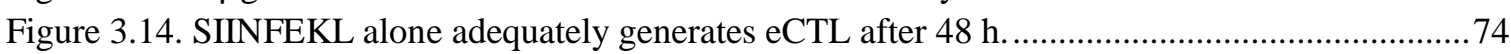

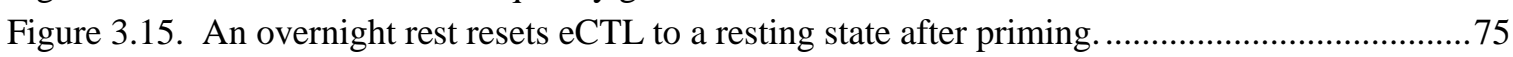

Figure 3.16. Priming SIINFEKL concentration may affect CTL functionality. ......................................

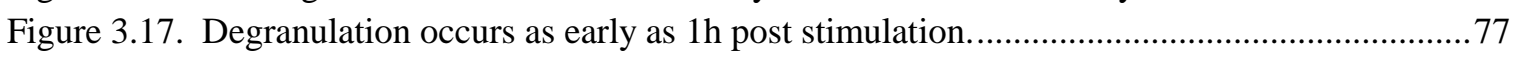

Figure 3.18. IFN $\gamma$ produced during eCTL activation is measurable after $2 \mathrm{~h}$ stimulation......................78

Figure 3.19. Microtubule depolymerisation due to Colchicine inhibits degranulation..........................79

Figure 3.20. The degranulation assay is not informative with a 20-hour drug pre-treatment................80

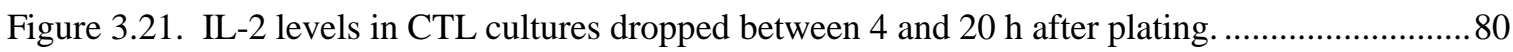

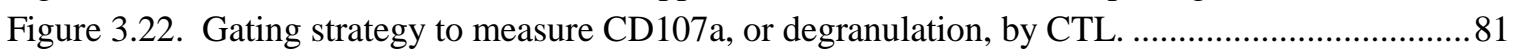

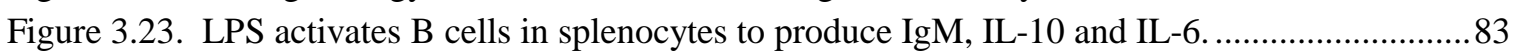

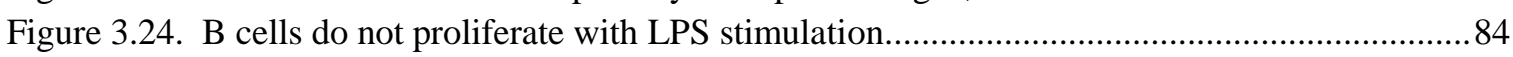

Figure 3.25. The effects of the protein synthesis inhibitor on B cells is detectable in this assay. .........85

Figure 4.1. The effects of screen-building set compounds on macrophage activation. ........................95

Figure 4.2. RAW 264.7 viability differences due to compound treatment and proliferative states......99

Figure 4.3. The effects of screen-building set compounds on macrophage phagocytosis..................101

Figure 4.4. The effects of screen-building set compounds on macrophage motility ..........................104

Figure 4.5. The effects of screen-building set compounds on helper $\mathrm{T}$ cell activity ...........................106

Figure 4.6. The effects of screen-building set compounds on $\mathrm{T}$ cell activation...................................109

Figure 4.7. The effects of screen-building set compounds on degranulation by CTL ..........................111

Figure 4.8. The effects of screen-building set compounds on B cell activity .......................................113

Figure 4.9. Activity profiles of the compounds in the screen-building compound set (opposite) .......114

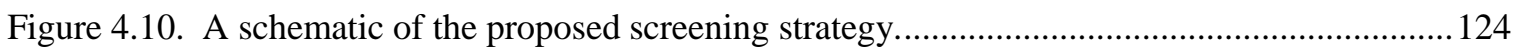

Figure 5.1. Testing concentrations for Compound E were determined by MTT data. ........................132

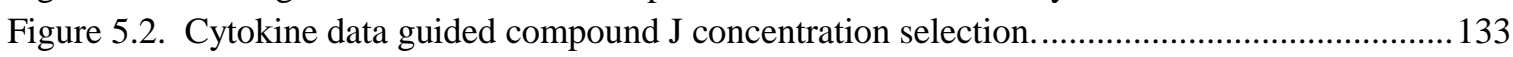

Figure 5.3. Compound F concentrations were selected by a combination of methods .........................134

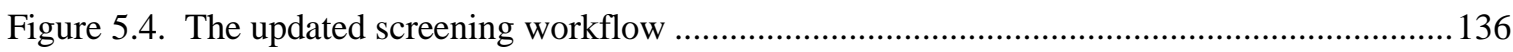

Figure 5.5. Screen outputs for Compound $\mathrm{L}$ as an example of screening data....................................137

Figure 5.6. Positive controls demonstrate that each assay was conducted successfully......................138

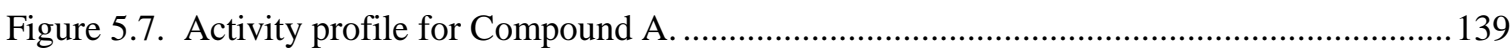

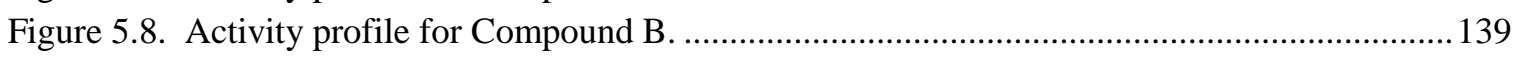

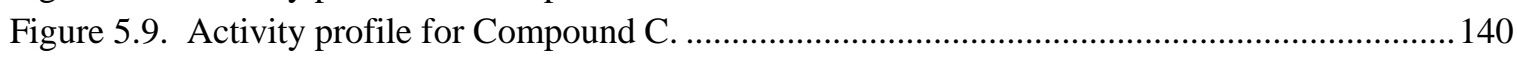




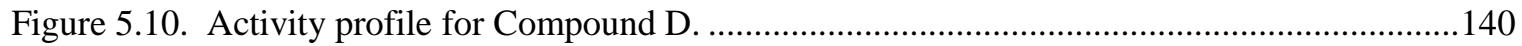

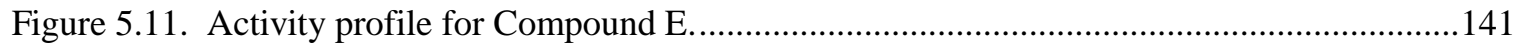

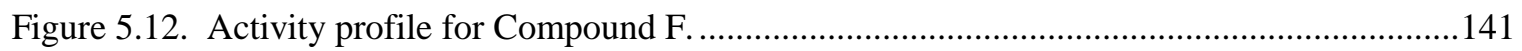

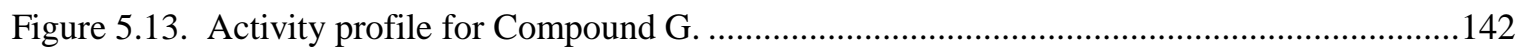

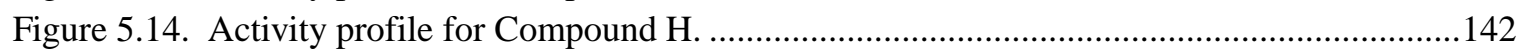

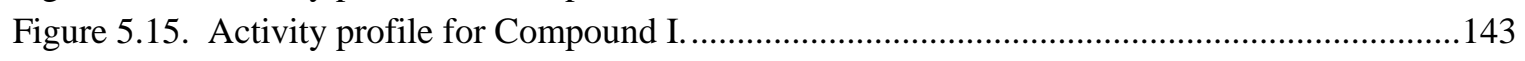

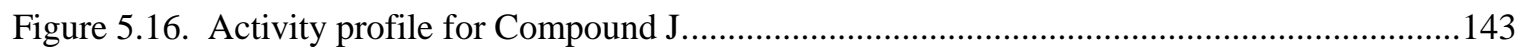

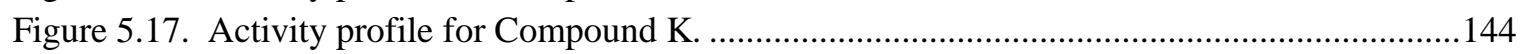

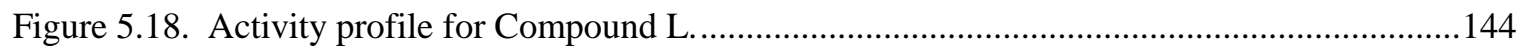

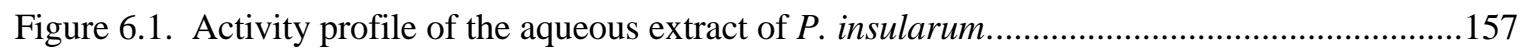

Figure 6.2. P. insularum alters IL-10 and IL-12 production independently of each other...................158

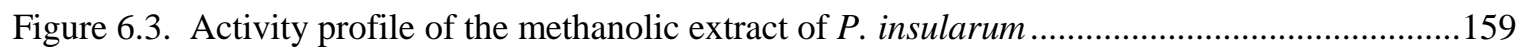

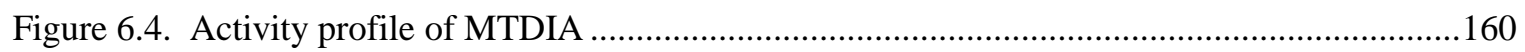

Figure 6.5. Additional MTA during macrophage activation exaggerates TNF $\alpha$ suppression by MTDIA

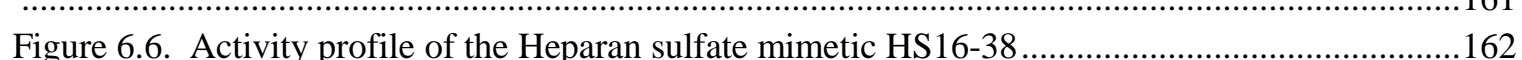

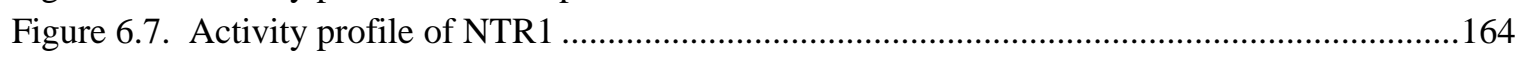

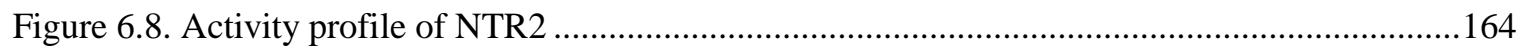

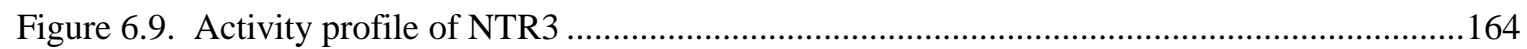

Figure 6.10. PelA alters macrophage cytokine production, and reduces viability of active T cells....165

Figure 6.11. Hamigeran G demonstrates activity against macrophages. ..........................................166

Figure 6.12. A hierarchical cluster shows compounds with shared activities .....................................168

Figure 9.1. Gating strategy to measure phagocytosis by RAW 264.7 by flow cytometry ...................201

Figure 9.2. Gating strategy to assess B and T cell proliferation during activation .............................202

Figure 9.3. Gating strategy to assess priming efficacy when generating effector CTL …...................203

Figure 9.4. eCTLs can be determined by their forward- and side-scatter properties ............................204

Figure 9.5. BMDM activation in the presence of screen-building set compounds ..............................206

Figure 9.6. Assessment of cell viability by Zombie dyes and MTT ...............................................207

Figure 9.7. Compounds were similarly toxic to stimulated and unstimulated B cells .........................208

Figure 9.8. Concentration identification for compound A using stimulated macrophages and Th cells

210

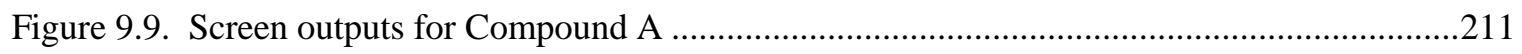

Figure 9.10. Concentration identification for compound B using stimulated macrophages and Th cells

212

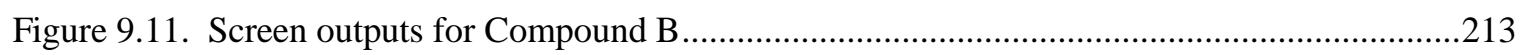

Figure 9.12. Concentration identification for compound C using stimulated macrophages and Th cells 214

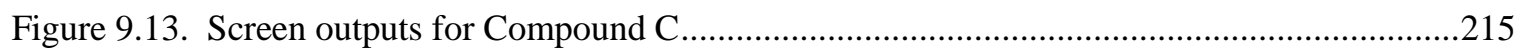

Figure 9.14. Concentration identification for compound D using stimulated macrophages and Th cells

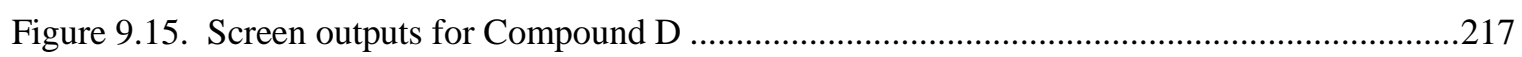

Figure 9.16. Concentration identification for compound $\mathrm{E}$ using stimulated macrophages and Th cells 218

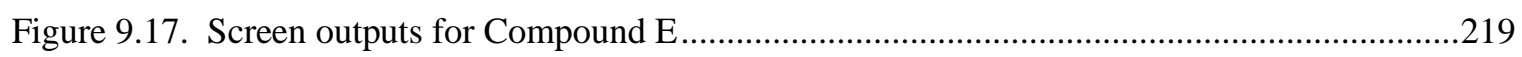

Figure 9.18. Concentration identification for compound $\mathrm{F}$ using stimulated macrophages and Th cells

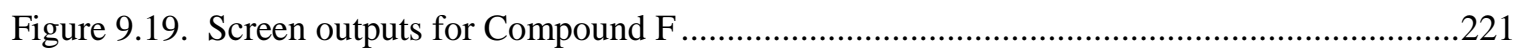

Figure 9.20. Concentration identification for compound $G$ using stimulated macrophages and Th cells

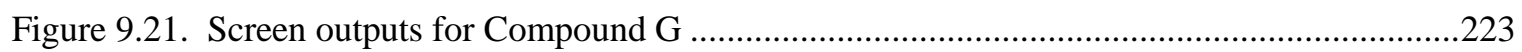


Figure 9.22. Concentration identification for compound $\mathrm{H}$ using stimulated macrophages and Th cells

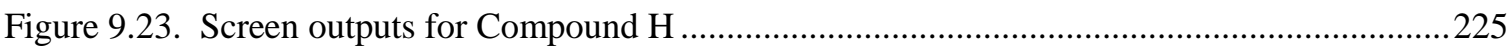

Figure 9.24. Concentration identification for compound I using stimulated macrophages and Th cells

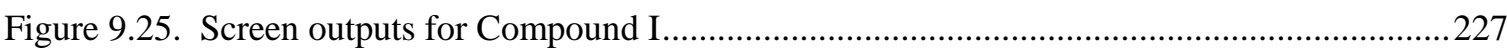

Figure 9.26. Concentration identification for compound J using stimulated macrophages and Th cells

Figure 9.27. Screen outputs for Compound J 229

Figure 9.28. Concentration identification for compound $\mathrm{K}$ using stimulated macrophages and Th cells 230

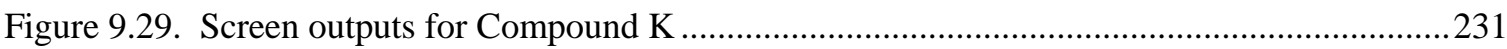

Figure 9.30. Concentration identification for compound L using stimulated macrophages and Th cells

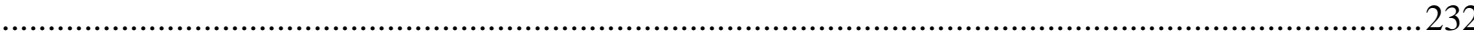

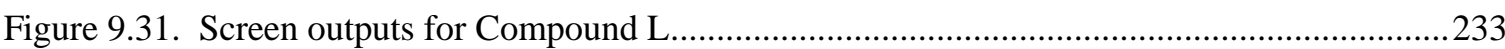

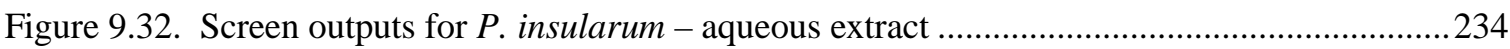

Figure 9.33. Concentration identification for the methanolic extract of $P$. insularum using stimulated

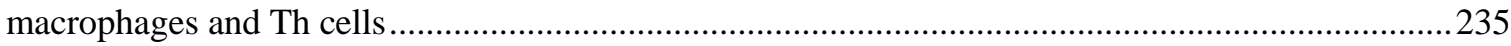

Figure 9.34. Screen outputs for $P$. insularum (methanolic extract) ……............................................236

Figure 9.35. Concentration identification for MTDIA using stimulated macrophages and Th cells..237

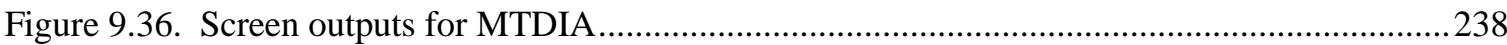

Figure 9.37. MTT of RAW264.7 cells is unaffected by MTA and MTDIA......................................2239

Figure 9.38. Concentration identification for HS16-38 using stimulated macrophages and Th cells. 240

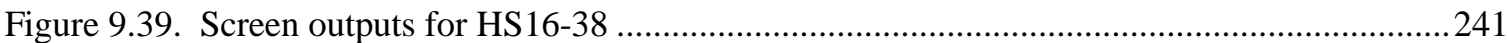

Figure 9.40. Concentration identification for NTR1 using stimulated macrophages and Th cells .....242

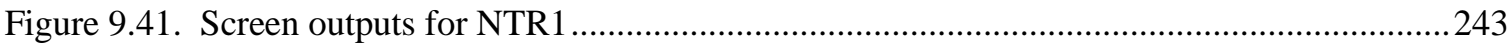

Figure 9.42. Concentration identification for NTR2 using stimulated macrophages and Th cells .....244

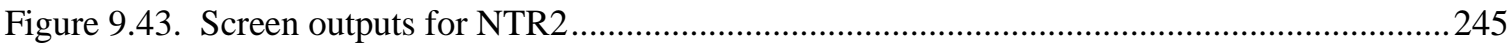

Figure 9.44. Concentration identification for NTR3 using stimulated macrophages and Th cells .....246

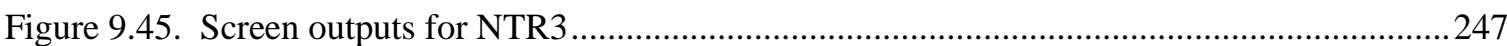




\section{List of Tables}

Table 3.1. eCTL generation with different priming and stimulation peptide concentrations.................76

Table 4.1. Screen building compound set: expected activities and concentrations used. .......................93

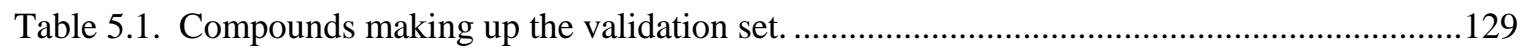

Table 5.2. Positive controls used in this chapter's experiments...........................................................138

Table 5.3. Summary of effects seen from screening compounds in the validation set.........................145

Table 5.4. Expected activities of validation set compounds ..............................................................146

Table 5.5. Observed and published immunomodulatory effects of test compounds.............................149 


\section{Abbreviations}

ADME absorption, distribution, metabolism and excretion

AMG aminoguanidine

APC antigen-presenting cell

BMDM bone marrow-derived macrophage

BPS bathophenanthroline disulfonate

Breg regulatory B cell

BTK Bruton's tyrosine kinase

CD cluster of differentiation

CFSE carboxyfluorescein succinimidyl ester

ConA concanavalin A

conc. concentration

COX cyclooxygenase

CTCM complete $\mathrm{T}$ cell media

CTL cytotoxic T lymphocyte

DC dendritic cell

DMSO Dimethyl Sulfoxide

dPBS Dulbecco's phosphate-buffered saline

eCTL effector cytotoxic T lymphocyte

ELISA enzyme-linked immunosorbent assay

Ex/Em excitation/emission

FCS foetal Calf Serum

FSC forward scatter

g gravity

GM-CSF granulocyte-macrophage colony-stimulating factor

gMFI geometric mean fluorescence intensity

ICH International Council for Harmonisation

IFN $\gamma \quad$ interferon gamma

IgG immunoglobulin $\mathrm{G}$

IgM immunoglobulin $\mathrm{M}$

IL interleukin

iNOS inducible nitric oxide synthase 


\begin{tabular}{|c|c|}
\hline LatA & Latrunculin A \\
\hline LPS & lipopolysaccharide \\
\hline MHC & major histocompatibility complex \\
\hline MT & microtubule \\
\hline MTD & maximum tolerated dose \\
\hline mTOR & mammalian target of rapamycin \\
\hline MTT & 3-(4,5-Dimethylthiazol-2-yl)-2,5-Diphenyltetrazolium \\
\hline NK & natural killer \\
\hline NO & nitric oxide \\
\hline NSAID & non-steroidal anti-inflammatory drug \\
\hline PAMPA & Parallel Artificial Membrane Permeability Assay \\
\hline PBS & phosphate-buffered saline \\
\hline PDD & phenotypic drug discovery \\
\hline PRR & pattern recognition receptor \\
\hline SA-HRP & streptavidin-horseradish peroxidase \\
\hline SLE & systemic lupus erythematosus \\
\hline SSC & side scatter \\
\hline STS & Standard Toxicity Studies \\
\hline TDAR & T cell-dependent antibody response \\
\hline TDD & target-based drug discovery \\
\hline Th & helper T cell \\
\hline Th1 & type 1 helper T cell \\
\hline Th17 & type 17 helper T cell \\
\hline Th2 & type 2 helper T cell \\
\hline TLR & toll-like receptor \\
\hline TMB & 3,3',5,5'-Tetramethylbenzidine \\
\hline $\mathrm{TNF} \alpha$ & tumour necrosis factor alpha \\
\hline Treg & regulatory helper T cell \\
\hline VUW & Victoria University of Wellington \\
\hline
\end{tabular}


Chapter 1:

Introduction 


\subsection{Preclinical drug discovery}

The 'standard model' of drug discovery and development can be divided into two major phases. The initial preclinical phase includes the discovery of novel compounds which show activity against a protein or cell process implicated in causing a diseased state. This phase also includes further work to assess the potency and selectivity of the compound against its target, and the mechanism of action if unknown. Pharmacokinetic studies investigate how the compound moves within the body, and the toxicology of the compound is also assessed. Compounds that pass these efficacy and safety assessments enter the clinical phase of drug discovery and development. In this second phase, the compound is tested in humans to further assess the safety and efficacy of the novel drug. If successful, the culmination of this work is the approval of a safe and effective new treatment for one of many diseases that affects human health and wellbeing.

\subsubsection{Where new drugs come from}

Natural products have provided relief from discomfort and disease: effective chemotherapies, analgesics and antibiotics have come from the natural world[1,2]. The search for novel therapeutic compounds from plants, marine sources, and microbes continues following the success of paclitaxel (Taxol), ziconotide (Prialt), penicillin and streptomycin. Given the proven utility of these natural products, traditional medicines are a useful source for identifying compounds with possible bioactivity. Extracts prepared from natural sources are tested for activity against living cells or organisms and, if the extract causes a change in bioactivity, individual compounds within the extract can be isolated and retested for activity. Alternatively, searching nature for novel molecules can also lead to the discovery of novel therapeutics after assessing bioactivity.

The chemical synthesis of compounds builds upon the efforts of sourcing compounds from nature. Once the structure of bioactive natural products have been elucidated, producing it in large quantities for further use relies upon the ability to synthesise the compound in the lab if the source material is not in plentiful supply[3]. During this process the structure of the original product can be altered, and this analogue can be tested for greater potency or higher specificity against its target. This can lead to a large number of synthetic analogues which differ from the parent molecule and produce distinct changes to cell activity. 
The chemical synthesis of bioactive compounds progressed as molecular biology technology advanced; the molecular causes of various diseases were discovered, which enabled structure-based drug design. This approach relies on the disease-causing proteins being identified, isolated, and modelled using crystallography, and compounds are then engineered to interact with these targets. Biologics, or biopharmaceuticals, are a relatively recent addition to the disease treatment arsenal. Utilising target-based drug design methodology, these molecules are generated after the disease-causing protein has been identified using biotechnological methods. Biologics are products made by cells which are manufactured on a large scale in the lab. For example monoclonal antibodies are a biologic, and anti-TNF $\alpha$ antibodies, which bind and neutralise TNF $\alpha$, have been used in the treatment of many inflammatory conditions, including rheumatoid arthritis[4].

Investigating existing drugs for activity against new targets is also a source of new disease treatments[5,6]. This drug repurposing, also known as drug repositioning, is appealing as the compounds are already approved for use in humans, and thus, this process may bypass safety assessments and other hurdles in drug development[7]. An example of a repurposed drug is duloxetine. This anti-depressant is approved for use for pain management and some cases of incontinence after demonstrating positive effects against these conditions[7-9]. Sometimes a compound's new application is discovered serendipitously during clinical trials such as the side effects observed during the testing of Sildenafil, which is a treatment for hypertension but is now known as Viagra. Generally, however, compounds are first retested for new applications in a laboratory[7].

Compound libraries are large collections of compounds which, for example, may be a collection of natural products or synthetic analogues. This library can be tested en masse using assays designed to identify potential drugs, and screening platforms have been developed for this purpose. These screening platforms are bioassays designed in mediumor high-throughput formats that allow for testing of hundreds to thousands of compounds simultaneously for the desired activity. Overall, there are two approaches to drug screening: target-based (reverse) and phenotypic (or classical) drug discovery. 


\subsubsection{Testing for compound activity}

Recently, the target-based drug discovery (TDD) approach has been the popular method for drug discovery as the advancement of molecular techniques in the past three decades has enabled the elucidation of disease-causing proteins[10]. With this approach, the protein that drives a disease state is identified and isolated, and binding assays identify compounds that interact with this target. This assay design is scalable as the process can be automated and run in low-volume 384, 1536, or 3456 well plates. These high-throughput formats can screen thousands of compounds within a short period of time. Compounds that demonstrate binding activity are hypothesised to modify the course of disease and are considered 'hit' compounds. Due to this narrow-scope approach to identifying hit compounds, off-target effects may be overlooked if compounds are not adequately tested in a range of assays that look for activity against other targets. These effects may induce undesirable changes to cells, and therefore the body, so understanding these effects is an important part of the remaining stages of preclinical drug discovery. Limitations of this approach include the need to have previously identified the cell mechanism that drives the disease state, and that compounds found to interact with the target may not be similarly active within the environment of an intact cell.

Phenotypic drug discovery (PDD) looks for compounds that induce desirable changes to cell activities. Different parameters can be measured from these cell- or organism-based assays, such as viability, morphology, gene expression or cell function. A hit compound is one that alters the measured biological activity(s) to resemble a desired state. For example, potential anti-cancer drugs can be identified by their ability to inhibit cellular proliferation. Because this method looks at changes to the activity of intact cells, hit compounds can be acting through a variety of targets. This broad-scope approach is one advantage of using PDD as treatments can be found for diseases where the cell activity is understood but the mechanism that causes this activity is unknown. Further work is sometimes necessary to understand the compound's mechanism of action, and the off-target effects of the compound need to be explored as its target may play an important role in other biological processes[11]. The PDD approach is slower than TDD as time is needed to observe biological changes, and the level of throughput is often lower in PDD as it commonly uses 96- and 384-well plate formats or whole organisms. However, it has the benefit of 
providing a greater depth of information if multiple cell activities are measured during screening.

\subsubsection{Pharmacodynamic and pharmacokinetic studies}

Hit compounds enter further rounds of testing. Pharmacodynamic studies investigate the mechanism of action, potency and selectivity of hit compounds. This work develops an understanding of the compound's effects on biological systems. The efficacy of compound activity is confirmed using in vivo disease models, looking for a reduced or altered disease state when the compound is administered. This in vivo work can overlap with pharmacokinetic investigations where the effects of the body on the compound are investigated. These experiments assess the absorption, distribution, metabolism and excretion (ADME) of compounds by looking for the compound in different tissues and organ systems. Several in vitro systems have also been established to produce preliminary pharmacokinetic data. For example, the Parallel Artificial Membrane Permeability Assay (PAMPA). This assay makes use of an artificial membrane that resembles the gastrointestinal tract. This is used to assess the absorption of compound via the gut and can give an indication on how the compound should be administered[12].

Compounds may be altered to improve their pharmacodynamic and pharmacokinetic properties, and those that do not perform well may be dropped from further investigation. This process is considered 'hit to lead' stage, and 'lead optimisation', where compounds can be refined to deliver improved activity across the tests run in this stage of drug discovery. This results in compounds with higher potency and specificity for their target, therefore an improved efficacy to deliver therapeutic effects. Before lead compounds can enter clinical trials to assess efficacy and safety in humans, preclinical safety and toxicity assessments need to be completed.

\subsubsection{Non-clinical safety assessments}

The International Council for Harmonisation of Technical Requirements for Pharmaceuticals for Human Use (ICH), is an association made of regulatory authorities and pharmaceutical industry members that promote best practices within the field of drug discovery. The ICH sets out guidelines for assessing the toxicity of compounds under investigation[13]; and additional toxicology assessments have been identified and incorporated into drug discovery programmes[14]. These toxicity studies ensure that off- 
target, potentially harmful activities of compounds are assessed before they are tested in human populations to ensure the safety of clinical trial participants.

Toxicity studies occur alongside pharmacokinetic and pharmacodynamic studies. These experiments look at the effects of a compound on different systems of the body. Preliminary toxicity studies can be done in vitro using cell lines that determine the concentrations of compounds that induce general cytotoxicity. This concentration is compared to the concentration required to produce desired changes to cell activities and it is preferable that desired activities are induced at much lower concentrations than cytotoxic effects.

The initial required toxicity study looks for compound-induced changes to DNA, or genotoxicity, such as DNA strand breaks and chromosomal aberrations using in vitro Ames or micronucleus assays. Any compounds found to be genotoxic would cause widespread damage to cells and tissues and would be unsafe for use. If no genotoxicity is seen, further tests look for changes to organs of the body. Cell lines from different organs can give preliminary insight into organ- and tissue-specific cytotoxicity, however in vivo animal experiments are used to assess compound toxicity.

First, a dose-escalation study is completed. This animal-based in vivo study is designed to identify the maximum tolerated dose (MTD) of compound where desired effects are observed without causing widespread morbidity or death. After treating animals with the MTD, tissues are analysed to evaluate the side effects a compound may cause. Cytological and histological analyses of vital organs is completed, including the heart, lungs, liver, kidney, brain, lymphatic organs, endocrine organs and blood. The site of administration is also assessed to look for signs of local damage. ICH Guidelines have also been established to assess the carcinogenic effects of compounds, as well as effects on reproduction. The data from these analyses are used to investigate toxicology: the compound-induced, detrimental changes to these tissues[14].

Other toxicity assays measure phototoxicity, or compound-induced light sensitivity, and phospholipidosis, to assess any intracellular accumulation of phospholipids caused by compound. Investigating mitochondrial toxicity is also recommended as this can result in tissue and organ damage. The Type II diabetes medicine, troglitazone, and the statin, 
cerivastatin were withdrawn from the market due to their toxic effects on mitochondria[1518].

\subsubsection{Immunotoxicology assessment}

Immunotoxicity describes compound-induced detrimental effects on the immune system and the ICH also has established guidelines for assessing the immunotoxicity of potential drugs[19]. This assessment, along with other toxicity assessments, is required before the compound can be tested in humans. The assessment of immunotoxicity is important as altering the immune system by drugs can introduce additional health concerns, such as immunosuppression, to people receiving the treatment.

Investigating immunotoxicity begins with a review of Standard Toxicology Studies (STS) data to determine the likelihood that compounds are affecting the immune system. These data come from the in vivo experiments used to assess toxicity in other tissues, as described in the previous section. STS data showing changes to leucocyte counts and populations from blood samples, changes to lymphatic organs (spleen, lymph nodes, bone marrow, thymus), changes to globulin levels, and/or an increased incidence of infection or tumour development indicates a compound is altering the immune system.

In these cases, further testing of the compound against the immune system is required. These compounds are often tested through the T cell-dependent antibody response (TDAR) assay to further evaluate the immunotoxicity of compounds. For this assay, Keyhole limpet hemocyanin (KLH) or sheep red blood cell (SRBC) preparations are injected into an animal. This is taken up by antigen presenting cells (macrophages, dendritic cells) that elicit T cell help to activate B cells. These B cells produce antibodies, the endpoint of the assay. If antibodies against KLH or SRBC are not detected, the compound suppresses the immune system in some way. The US Environmental Protection Agency recommends running this assay for 28 days, using at least eight animals[20]. This assay involves many cells of the immune system, and while a blunt tool, it has been shown to be a sensitive assessment of immune function[21].

The TDAR assay is used for most immunotoxicity studies, unless the STS data suggest that compounds alter immune cell populations which are not involved in generating an antibody 
response. For example, if natural killer (NK) cells or Cytotoxic T lymphocytes (CTLs) populations appear to be altered by compound treatment, as determined by phenotyping blood samples from STS studies, the recommendation is to complete more appropriate experiments that assess changes to these cells.

\subsection{The impact of immunomodulation}

\subsubsection{Altering a functional immune system}

The immune system is a complex network of cells and their activities which, together, maintain health by identifying and eliminating pathogens and by destroying abnormal cells of the body. Drugs can alter the activities of immune cells to improve their functions in maintaining health, or, if these changes are unwanted, they can have detrimental repercussions for human health. This potential for adverse immune-altering effects explains the necessity for immunotoxicology assessments of potential drugs.

Though the structure of the compound under investigation may be well characterised and the specificity of the compound for its target is understood, the target may also be involved in immune cell functions. Thus, the compound may unintentionally alter the immune system. Other immune altering activities occur because many of the cellular pathways that immune cells use are common to all cells. For example, anti-mitotic compounds used for treating proliferating cancers result in immunosuppression due to the inhibition of lymphocyte proliferation required for clonal expansion during an adaptive immune response. This immunosuppression can increase susceptibility to infection, as seen with Paclitaxel[22]. It is these off-target effects of compounds that are a concern during drug development because these effects are undesirable and can introduce new risks to drug recipients.

Immunosuppression is not the only concern, some drugs have been involved with inducing autoimmunity, where the immune system begins to mount an attack against normal cells of the body. Hydralazine, prescribed to treat hypertension, can induce lupus erythematosuslike conditions where the body produces antibodies against nuclear proteins[23,24]. Additionally, other therapeutics have been observed to stimulate the immune system; this was seen with TGN1412, a monoclonal antibody that was intended for treatment of arthritis 
and leukaemia. During the phase 1 clinical trial with healthy volunteers, TGN1412 activated the immune system causing a cytokine storm, and the resulting inflammation caused significant organ damage to the six men who received TGN1412[25-28].

The standard process for assessing immunotoxicity is adequate for many drugs in development, though several reviews acknowledge the difficulty in establishing protocols and standards for immunotoxicology assessment when the TDAR assay is not suitable[14,29-31]. The TDAR assay has a limited scope for observing changes to the immune system as the endpoint of the assay is solely IgM and IgG antibody production. If an effect is seen, the assay does not identify which cell type is altered by compounds, nor does it identify other immune processes that might be affected, e.g. CTL activity. To appreciate the wide range of the immune system's activities, a concise overview, which covers the main cell types and their functions, follows.

\subsubsection{The innate immune system}

The innate immune system consists of phagocytes (dendritic cells, neutrophils, monocytes and macrophages), granulocytes (neutrophils, eosinophils, basophils and mast cells), and innate lymphocytes (e.g. natural killer cells, innate lymphoid cells). These cells are found throughout the body and, due to their quick activation, respond rapidly to infection. As first responders these innate immune cells detect infection or cellular damage, induce inflammation, recruit other immune cells to sites of inflammation, or kill pathogens directly. Some innate immune cells (i.e. dendritic cells, monocytes and macrophages) are also antigen presenting cells (APCs), which are essential in the activation of the adaptive immune system.

Macrophages are important in inducing inflammation but also in directly controlling infection. These cells reside in tissues and are often the first immune cell to encounter pathogens or detect tissue injury. Macrophages, like other innate immune cells, are activated via pattern recognition receptors (PRR), which bind to molecular motifs that are commonly associated with pathogens. One example is Toll-like receptor 4 (TLR-4), which recognises lipopolysaccharide (LPS), a cell wall component of gram-negative bacteria. Macrophages produce cytokines upon TLR-4 activation, including TNF $\alpha$, IL-12 and IL10. TNF $\alpha$ is a key cytokine involved in inflammation and causes local and systemic effects including vasodilation, increased vascular permeability, and fever all of which allow 
leucocytes to enter tissues and creates conditions less favourable for pathogen survival; these conditions also cause pain. IL-12 is a pro-inflammatory cytokine that drives a Th1 bias when $\mathrm{T}$ cells are activated whereas $\mathrm{IL}-10$ is a regulatory or immunosuppressive cytokine that limits the propagation of inflammation.

The cytokine environment dictates the type of immune response that is generated and its subsequent effector functions. A good example of how the cytokine profile of innate immune cells correlates to their effector functions is macrophage polarisation. The production of TNF $\alpha$ and IL-12 is associated with M1 polarisation, which is a proinflammatory macrophage phenotype. With different stimuli, or as infections resolve, macrophages can adopt other phenotypes such as an M2, anti-inflammatory phenotype. The M2 state is broadly characterised by higher IL-10 and reduced IL-12 production, which is believed to promote the resolution of inflammation and wound healing. However, M2 can be further divided into M2 subtypes depending upon the initiating stimulus and each subtype retains its own unique phenotype. Macrophages are very plastic and can shift between these phenotypes depending on signals from their environment and other immune cells[32,33].

Activated macrophages also produce reactive oxygen and nitrogen species, which destroy pathogens by damaging microbial DNA[34,35]. Alongside this, macrophages can directly kill pathogens by phagocytosis, which limits the spread of infection. Once endocytosed, pathogens are contained within a phagosome, which then fuses with lysosomes; lysosomes contain enzymes that destroy phagocytosed pathogens. Phagocytosis is also important for wound repair allowing macrophages to clear cell debris and promote healing[35].

Phagocytosis is an important function for dendritic cells (DCs), monocytes, and macrophages to fulfil their role as professional antigen presenting cells (APCs). When engulfed pathogens are broken down inside APCs, peptide fragments (i.e. antigen) from these pathogens are presented on MHC class II molecules on the cell surface. Dendritic cells presenting antigens will migrate to lymph nodes or the spleen and interact with helper $\mathrm{T}$ cells. If the helper $\mathrm{T}$ cell recognises the specific antigen presented by the dendritic cell, the helper T cell becomes activated. Although monocytes, macrophages and B cells can also act as professional APCs, dendritic cells are proficient at antigen cross-presentation, where peptides from phagocytosed material are presented on MHC class I to activate 
cytotoxic T lymphocytes (CTL)[36]. The activation and function of T cells will be covered in more detail in the next section (1.2.3)

Neutrophils are very proficient at phagocytosis and respond to infection in large numbers, but they lack APC activity. Instead, these innate cells act to control infection or inflammation quickly. Once a pathogen has been phagocytosed by a neutrophil, these cells generate reactive oxygen and nitrogen species to destroy the pathogen. These cells can also quickly release preformed anti-microbial products stored in granules through a process called degranulation, and these anti-microbials can destroy extracellular pathogens[37]. Degranulation is an activity shared amongst granulocytes. Eosinophils release products that combat helminth infections, whilst basophils and mast cells have been associated with releasing histamine and heparan to promote inflammation at local sites[38]. Natural killer (NK) cells are innate immune cells but share a role with CTLs by responding to cells of the body that are in an abnormal state. These cells recognize MHC Class I molecules (i.e. molecules that identify 'self'), and this recognition suppresses NK cell killing activity. In contrast, if an NK cell encounters a cell in a stressed state, where MHC is downregulated, the NK cell is activated and kills the cell through degranulation, resulting in the release of cytolytic enzymes[39]. The NK cell has receptors for other stress signals, enhancing its ability to kill abnormal cells in a broad but non-specific manner in contrast to the CTL, which kills in an antigen-specific manner[40].

Together, these innate immune cells can mount a quick but relatively unspecific response to infection with their activities geared towards broad types of microbe (e.g. bacteria, fungi). If the innate immune system cannot resolve the infection alone, the adaptive immune system will be induced and respond to infection in a more targeted, specific manner.

\subsubsection{The adaptive immune system}

Naïve T cells circulate through secondary lymphoid organs, such as the spleen and lymph nodes. During an infection, APCs (especially DCs) migrate to these organs and seek out the cognate $\mathrm{T}$ cell which has a $\mathrm{T}$ cell receptor (TCR) that specifically recognises the antigen presented on an MHC molecule on the APC. There are two main type of T cells: helper T (Th) cells and cytotoxic T cells (CTL), and these cells differ not only in how they are activated but also in their effector functions. 
For Th cells, antigens must be presented on MHC class II molecules for recognition by their TCR. Upon recognition and activation, Th cells undergo rounds of proliferation and differentiate into one of many Th subtypes (e.g. Th1, Th2, Th17, and Treg) based on the cytokine milieu[41,42]. Each Th subtype produces a distinct set of cytokines to promote the specific immune cell activities best-suited to the type of infection present. For example, Th1 cells produce IFN $\gamma$ which acts on immune and non-immune cells to encourage an antiviral (or anti-intracellular pathogen) response. IFN $\gamma$ encourages macrophages to produce nitric oxide (NO) and promotes phagolysosomal fusion. IFN $\gamma$ also supports CTL activity and can have direct antiviral properties[43]. In contrast, Th2 cells promote humoural immunity and wound healing via IL-4 production. This cytokine promotes B cell proliferation and differentiation into antibody-producing cells, and promotes eosinophil, basophil and mast cell activities[38,44]. Pro-inflammatory Th17 cells produce IL-17A, which drives neutrophil responses, and is associated with the maintenance of mucosal immunity and inflammation[45-48]. The last main subset of Th cells is Tregs which produce IL-10. This cytokine is immunoregulatory and dampens down many activities to regulate the immune response. The different subsets of Th cells regulate each other, creating a bias towards a particular Th subset during each specific immune response.

Whereas the TCR on Th cells interacts with MHC Class II on professional APCs, the TCR on CTLs interacts with MHC Class I. MHC class I is a surface-bound complex found on all cells which binds peptides generated from the degradation of cytosolic proteins by the proteasome. In other words, MHC class I molecules display the endogenous proteins of all cells[49]. During intracellular infection, peptides from pathogens are presented on MHC class I indicating the cell is infected[50]. Naïve CTLs need to be activated by APCs before they can respond to these infected cells. For this, DCs phagocytose intracellular pathogens and cross-present pathogen antigen on MHC class I. These APCs then migrate to secondary lymphoid organs to activate naïve CTLs that specifically recognise this pathogen.

Upon activation, CTLs proliferate and exit secondary lymphoid organs to search for cells presenting the antigen on MHC class I, i.e. infected cells. The IFN $\gamma$ produced during infection promotes the upregulation of MHC class I and facilitates CTL recognition[51]. When a CTL encounters an infected cell, it releases cytolytic granules towards the target via degranulation. These granules contain perforin, granzyme and granulysin, which together induce apoptosis in the target cell, limiting the spread of intracellular pathogens. 
The CTL can move on to another infected cell and repeat the killing process, and because a CTL only targets the infected cell, this activity is precise and efficient[52]. In addition to controlling viral or intracellular pathogen infections, CTLs recognise and destroy tumour cells if they present abnormal, tumour-associated peptides on their surface. CTLs also destroy cells that have foreign MHC class I molecules, and this recognition of foreign MHC molecules is the underlying mechanism of transplant rejection[53,54].

Active B cells are best known for producing antibodies although more recent work suggests that these cells have other effector functions as well, including immunoregulation[55,56]. Antibodies bind to pathogens directly and can neutralise the invader, mark them for phagocytosis in a process called opsonisation, or lyse pathogens by activating the complement system[57]. B cells are activated via the B cell receptor, and undergo rounds of proliferation before becoming antibody-producing plasma cells[58]. B cells also express TLRs, which provide an alternate route of activation and antibody production[59]. The first antibody isotype B cells produce is IgM, and B cells can produce this antibody in a T cell-dependent or independent manner. When a B cell presents antigen via MHC class II to a Th cell the signals from the Th cell induce class switching, resulting in the production of other antibody isotypes (e.g. IgG). These isotypes have different activities and distribution throughout the body, and these activities are best suited for different types of infection. B cells also produce a variety of cytokines, though the types of cytokines they make depends on their subtype[60]; some B cell subtypes enhance immune responses, whilst others play a regulatory role[55,56,60].

Though slower to respond, adaptive immune cells act in a specific manner against a pathogen and can form 'memory' cells. After activation during an immune response, memory cells enter a quiescent state in secondary lymphoid organs or peripheral tissues (e.g. gut or lung). Upon reactivation, these immune cells can react quickly in a highly specific and efficient manner as they have been fine-tuned during their initial activation. Adaptive immune cells support the activity of innate immune cells when pathogen control requires a precise response. There is interplay between the two branches of the immune system with innate immune cells inducing the adaptive immune system, which responds in turn by boosting and fine-tuning the activities of all immune cells.

If any of these immune cell activities are altered or suppressed, there may be a significant impact on the ability of immune cells to respond to infection, injury, or abnormal cells in 
the body. Alternatively, if activities are elicited or enhanced, inflammation and other damaging pathways may be triggered. Therefore, as previously discussed, it is important that newly developed drugs do not alter the immune system to avoid introducing new health concerns to patients. Whilst immunotoxicity assessment with the TDAR assay covers many of these cell activities with one final readout, it does not assess the contributions of each immune component nor does it evaluate bactericidal activity, cytokine release, or CTL and NK activity. These latter activities are only investigated if STS data show immunotoxicity which is not reflected in the TDAR assay. Thus, there is scope to facilitate immunotoxicology assessment by identifying the immunomodulatory properties of compounds with more specificity to guide further investigations.

\subsubsection{Altering a dysfunctional immune system}

Not all immunomodulation is undesirable. While the immune system is normally responsible for maintaining health, it can go awry and itself become the cause of disease. This is the case with chronic inflammatory and autoimmune conditions, which afflict a significant portion of the population[61]. These conditions are caused by aberrant immune activity but remain difficult to treat due to the complex nature of the immune system. Compounds that correct the immune dysfunction driving these diseases are desirable, and changes to specific disease-causing pathways have been shown to be more effective than generalized immune suppression for sustained treatment of immune-mediated disorders. As with other compounds with therapeutic potential, the aim is to leave normallyfunctioning immune cells intact. Some current treatment options for various immunemediated diseases including Crohn's disease, gout, multiple sclerosis, vitiligo, systemic lupus erythematosus, leukaemia, and transplant rejection are discussed below to demonstrate the range of compound activities that are currently used to control a dysfunctional immune system.

Though the cause of Crohn's disease is poorly understood, symptoms of disease are caused in part by inflammatory macrophage activity, but also by the dysregulated activities of $\mathrm{T}$ cells, NK cells and innate lymphoid cells[62-65]. To alleviate this chronic inflammation patients are often given Mesalamine, a non-steroidal anti-inflammatory drug (NSAID) COX inhibitor that is active in the gut[66]. COX inhibitors block the synthesis of prostaglandins which inhibits macrophages and other immune cells from producing 
mediators of inflammation. NSAID are used in many other inflammatory diseases, such as rheumatoid arthritis and gout.

Gout is another chronic inflammatory disease characterised by swollen joints caused by elevated uric acid levels in blood. Colchicine is prescribed when NSAIDs are not well tolerated by patients. Colchicine destabilises microtubules which results in reduced inflammation. When the cytoskeleton is disrupted, innate immune cells have reduced capacity to establish inflammation due to their inability to secrete cytokines, histamine and heparin. These cells also have a limited capacity to receive pro-inflammatory signals as the receptors cannot be mobilised to the surface. Microtubule disruption by colchicine also inhibits the ability of immune cells to migrate to sites of inflammation where they would otherwise propagate this unwanted activity. Colchicine remains a prescribed treatment for this inflammatory disease for these reasons[67].

Multiple sclerosis is an autoimmune disease where the immune system attacks the central nervous system. It is caused by Th cells that recognise specific myelin proteins in the myelin sheath that surrounds nerve cells. These self-reactive Th cells recognise myelin as foreign and coordinate the destruction of oligodendrocytes, the cells that provide the myelin sheaths. Different treatment strategies have been developed to control this disease including the modulation of inflammation, inhibition of migration, and lymphocyte depletion. Glatiramer acetate has been found to alter macrophage activation from a proinflammatory IL-12-producing state to a more anti-inflammatory state characterized by IL10 production. Additionally, glatiramer acetate has been shown to enhance Treg numbers and function, reducing disease progression[68-70]. Natalizumab inhibits the migration of $\mathrm{T}$ cells from the bloodstream into tissues, thus blocking them from entering the central nervous system. Because the adaptive immune system has a clear role in driving disease relapses in MS, alemtuzumab, which specifically depletes circulating lymphocytes, is very effective but leaves recipients susceptible to infection.

Autoreactive CTLs are implicated in type 1 diabetes, where the immune system attacks the pancreatic beta cells which produce insulin[71-73]. The disease is managed with insulin replacement therapy. Vitiligo is another disease mediated by aberrant CTLs, where these immune cells attack melanocytes causing a loss in skin pigmentation[74,75]. Steroids and 
tacrolimus are prescribed to patients with vitiligo to generally suppress the immune system, or $\mathrm{T}$ cells, respectively.

B cells are implicated in various diseases such as systemic lupus erythematosus where autoreactive B cells produce autoantibodies[76]. These antibodies target and bind DNA or nuclear proteins which results in cell death and tissue injury causing pain and fatigue[77]. Innate immune cells also play a role in disease progression: the impaired phagocytic activity of macrophages leads to accumulations of apoptotic cells[78]. It has been suggested that this promotes disease by continually exposing nuclear proteins to the immune system. No cure exists for systemic lupus erythematosus; symptoms are usually managed with anti-inflammatory NSAIDs.

B cell cancers result in abnormal immune function, fever, weight loss and fatigue. The anti-CD20 therapy, rituximab, is a monoclonal antibody that targets and neutralises B cells, and it is currently used to treat these cancers[75]. Ibrutinib is also used to treat this disease. This drug is a Bruton's tyrosine kinase (BTK) inhibitor, which reduces B cell activation leading to their eventual apoptosis[80].

Finally, there is currently a major need for immunomodulation and/or immunosuppressants to inhibit transplant rejection. CTLs will recognise transplanted organs as foreign due to the presence of foreign MHC Class I proteins and other potential protein polymorphisms. To inhibit rejection, the immune system (especially T cells) needs to be suppressed, and this suppression is achieved through several mechanisms. Some agents block the T cell growth factor IL-2 (e.g. glucocorticoids), which suppresses $\mathrm{T}$ cell activation and proliferation. Others inhibit proliferation by blocking DNA synthesis (e.g. azathioprine) or block signalling pathways involved in T cell (and general lymphocyte) activation (e.g. rapamycin, an mTOR inhibitor).

These are a few examples of conditions where the dysfunctional activities of immune cells cause disease. Different immune cells play key roles in the progression of immunemediated disorders calling for different forms of treatment. While some treatments target problematic cells, other conditions are treated by broadly suppressing the immune system. Moreover, for some conditions, such as type 1 diabetes, no immune-targeting treatment is available. 


\subsubsection{A need for novel immunomodulators}

There is need for new immunomodulatory drugs. Several autoimmune conditions can currently only be treated with broad range immunosuppression, whilst others have no treatment options. In other cases, treatment options can be improved by targeting problematic cells whilst leaving other immune cell processes intact, improving the wellbeing of the patient. For example, vitiligo treatments may be more effective if CTLs were specifically targeted. At the same time, it is important to consider that many immune disorders are caused by multiple aberrant immune cells, so a multipronged attack may be efficacious for those conditions. These themes have been a topic of conversation over the past decade, and are important considerations whilst searching for novel immunomodulatory therapies[81-83].

\subsection{Identifying immunomodulation to improve drug discovery}

Immunomodulation can both be a negative and positive outcome of a compound's activity, and the outcome is dependent on the application of the drug in development. Detecting immunomodulation for investigating the immunotoxicity of compounds, and for finding new treatments for immune-mediated disorders, is similar. Therefore, in this project, I propose a new screening platform by which the immunomodulatory properties of novel compounds can be identified in a systematic, broad-scope manner that identifies compounds that alter the activities of a variety of immune cells and cell functions.

The standard method to assess immunotoxicity comes with a level of uncertainty and high costs. These assessments are done in animals after compounds have been optimised for intended activity. Whilst these assessments serve the purpose of identifying compounds that affect the immune system, it is completed late into the drug development process with a significant loss of time and money if the compound does not pass immunotoxicology assessments. Assessing the immunomodulatory activity of compounds earlier in the drug discovery process could mitigate some risks in drug discovery. 
For some research institutions it may be more desirable to assess immunomodulatory activity of compounds at earlier stages of drug discovery as a method to simply identify bioactivity. For example, institutions exploring novel compounds may want to narrow down the number of compounds to investigate and could do this based on the effects observed against the immune system. Compounds could be ruled out due to an early indication that they induce inflammation or are cytotoxic, whilst other compounds may be identified as a potential treatment for an immune-mediated disorders and this could guide further compound investigations. Or, this screen can simply be used to identify compounds that exhibit bioactivity as many common cell processes are utilised by the immune system.

Another concern in drug discovery is the use of animals in research. To minimise the associated costs and ethical concerns of running in vivo experiments, several workshops involving industry members have discussed assessing immunomodulation with in vitro assays, especially for immune cell cytotoxicity assessments[84,85]. In vitro assays for immunomodulation can be assembled into a screening strategy to serve these identified needs. It could be performed either as the first screen to identify immunomodulators, or as an immunotoxicity counter-screen for compounds intended for other applications.

To summarise, discovering novel immunomodulators shares similarities with assessing the immunotoxicological effects of compounds under investigation. A screening platform that assesses immunomodulation could facilitate the investigation of a compound's ability to alter the immune system in these two scenarios. A search of the literature suggests no broad-scope screening platform exists to assess the immunomodulatory activities of compounds.

\subsubsection{Considerations for screen design}

For a screening approach to work, assays need to adequately represent cell activities whilst being sensitive to alteration by test compounds. The phenotypic approach suits identifying immunomodulators as cell-based assays, where immune cells have been stimulated to elicit activity. This involves many cell mechanisms against which a compound could act, simultaneously, to induce immunomodulatory effects. Additionally, data from these assays are direct measurements of changes to immune cell activity caused by compounds. 
Changes to several immune cell types should be assessed to offer valuable, broad-scope insight into immunomodulation by compounds. By doing this, a more detailed overview of immunomodulatory activity can be generated to guide the subsequent investigations into compound activity, and application or safety.

Key considerations when building a screening strategy/platform include the need for assays to be relevant to disease states. This consideration is addressed by including assays that measure immune cell functions (e.g. viability, cytokine production) as the assessment of cell function relates best to a well-functioning, or dysfunctional, immune system. The assays also need to be cost-effective so that many iterations of the experiments can be run to routinely screen many compounds. This, as well as using simple assay design requiring common lab equipment improves the accessibility of the screening platform.

Assessing compound activity against a broad range of cell activities with a screening strategy can serve many needs. It can provide early insight into a compound's effects against the immune system. This can identify potentially harmful compounds, and compounds that have potential to treat immune-mediated disorders.

\subsection{Aims and objectives}

This thesis describes the process behind developing a screening strategy to identify the immunomodulatory activities of compounds. Towards this aim, the objectives of this thesis are to:

1. Select, and optimise or develop assays that assess immunomodulation.

2. Devise a screening strategy which brings these assays together into an efficient workflow.

3. Evaluate the screening strategy's performance through testing its ability to adequately identify immunomodulators.

4. Use this screen to characterise the immunomodulatory activity of novel compounds. 
Chapter 2:

Methods 
This chapter contains the optimised experimental methods developed in this thesis. The optimization process that led to these methods is fully detailed in Chapter 3: Optimising assays to detect immunomodulation.

\subsection{Compound preparation}

Compounds were prepared to stock solutions of, or close to, $10 \mathrm{mM}$ in vehicles (solvents) recommended by the supplier (see appendix 9.2, pg 198). As some compounds were not yet optimised for potency, high treatment concentrations were sometimes tested throughout this thesis. To account for the effects that the vehicle itself can have in biological systems, cells were treated with compounds and the equivalent volume of vehicle served as a control. Compound activities were then represented as a percentage of the vehicle control where the amount of solvent was equivalent in both conditions. That is, vehicles were titrated alongside test compounds.

To identify the concentrations of compounds to run through the screening strategy, a concentration selection process has been designed. After an initial round of testing using a wide range of concentrations of compounds, MTT and ELISA data were used to narrow down the concentration range for subsequent assays. Section 5.3.1 (pg 130) details the precise guidelines on selecting the concentrations to use in the assays.

\subsection{Mice}

C57BL/6J mice were maintained at Victoria University of Wellington's (VUW) animal facility; transgenic OT-1 (B6.SJL-Ptprc ${ }^{\mathrm{a}}$ background) mice were purchased from the Malaghan Institute of Medical Research (Wellington, New Zealand). All mice were housed in a specific pathogen-free (SPF) facility at VUW and handled in a laminar flow hood to maintain SPF-like conditions. Ethical approval was obtained prior to experiments under VUW Animal Ethics Committee's protocol 0000025295. The mice used in this project were between 10 - 14 weeks old. 


\subsection{Cell culture}

\subsubsection{General cell culture}

To simplify the programme of work, all cell culture work shared the same medium, culture conditions, and handling. For assays requiring cell culture, cells were handled in a Class II biosafety cabinet (Labconco, MO, USA), and all treatment and stimulation steps were also completed in sterile conditions. Complete T Cell Media (CTCM, see appendix 9.1) was used for all cultures, and cells were cultured at $37^{\circ} \mathrm{C}$ with $5 \% \mathrm{CO}_{2}$. To pellet cells, they were centrifuged at $300 \times \mathrm{g}$ for 4 minutes. When required, cells were counted using $0.4 \%$ trypan blue dye (Gibco via Thermo Fisher, MA, USA) and bright cells that excluded the blue dye were considered live. Live cell concentration and \% viability was determined with a haemocytometer (Neubauer) and microscope (Olympus CX41, Tokyo, Japan) using the formulas given below.

$$
\begin{aligned}
& \text { cell concentration }(\text { cells } / m L)=\text { average cells per square } \times \text { dilution factor } \times 10^{4} \\
& \text { viability }(\%)=\left(\frac{\text { number of live cells }}{\text { total number of cells }}\right) \times 100
\end{aligned}
$$

\subsubsection{The monocyte/macrophage RAW264.7 cell line}

The adherent monocyte/macrophage RAW264.7 cell line is virally-induced immortalised cell line of BALB/c mouse origin. Stocks used in this project were supplied by VUW. Cells were grown in T75 flasks (Falcon by Corning, NY, USA) seeded with $1 \times 10^{6}$ cells in $15 \mathrm{~mL}$ CTCM. Cultures were passaged every three days when cells reached $80 \%$ confluency. For this, flasks were rinsed with calcium- and magnesium-free PBS before detaching cells with a rubber scraper (Falcon) into $5 \mathrm{~mL}$ fresh PBS. After transferring into a $15 \mathrm{~mL}$ conical tube, cells were centrifuged, supernatants were discarded, and the resulting pellet was resuspended in $1 \mathrm{~mL}$ PBS for counting. For long term storage, $2 \times 10^{6}$ cells in 10\% DMSO/FCS were maintained in liquid-phase nitrogen. For all experiments, cells were used between passage numbers 10 - 25.

\subsubsection{Bone marrow derived macrophages (BMDM)}

Femurs and tibias were removed from mice following euthanasia by $\mathrm{CO}_{2}$ asphyxiation. After removing any attached tissue from bones, epiphyses were cut off and bones were flushed with cold calcium- and magnesium-free dPBS (via 23G \& 25G syringes), collecting 
the marrow in $50 \mathrm{~mL}$ conical tubes. After centrifugation and discarding supernatants, the cell pellet was loosened and resuspended in $2 \mathrm{~mL} \mathrm{RBC} \mathrm{lysis} \mathrm{buffer} \mathrm{(Sigma-Aldrich)} \mathrm{for}$ two minutes at room temperature to remove red blood cells. This lysis step was quenched with the addition of $8 \mathrm{~mL}$ CTCM before washing cells by centrifugation. Cells were counted, and cell concentration was adjusted to $1.2 \times 10^{6}$ cells $/ \mathrm{mL}$ in CTCM. Twenty - thirty $\mathrm{mL}$ aliquots of this cell suspension were distributed amongst T75 flasks and incubated. The following day, rmIL-3 (Prospec, Rehovot, Israel) and rmGM-CSF (Prospec) were added to flasks to a final concentration of $5 \mathrm{ng} / \mathrm{mL}$ of each cytokine. Five $\mathrm{mL}$ aliquots of the cell suspension were transferred to $90 \mathrm{~mm}$ diameter sterile petri dishes, leaving behind adherent cells which were discarded. After four days of incubation, culture media was refreshed by adding $5 \mathrm{~mL}$ of CTCM with $5 \mathrm{ng} / \mathrm{mL}$ rmIL-3 and $5 \mathrm{ng} / \mathrm{mL}$ rmGM-CSF. After a further four days incubation, media and any non-adherent cells were discarded, and plates were rinsed with room temperature calcium- and magnesium-free dPBS. The remaining adherent cells, BMDM, were then harvested from plates by rinsing with ice-cold dPBS. BMDM were collected in conical tubes, washed via centrifugation, and counted before diluting in CTCM for use.

\subsubsection{Macrophage activation by a combination of IFNY and LPS}

RAW 264.7 (50,000 cells) or BMDM (100,000 cells) were seeded into wells of a flat-bottomed 96-well plate (Falcon) and cells were primed with $20 \mathrm{U} / \mathrm{mL}$ rmIFN $\gamma$, working to a volume of $140-150 \mu \mathrm{L} /$ well. After an $18 \mathrm{~h}$ incubation, cells were treated with compounds and were stimulated with $200 \mathrm{ng} / \mathrm{mL}$ LPS (E. coli, Sigma-Aldrich), working to a final well volume of $200 \mu \mathrm{L}$. For unstimulated conditions, rmIFN $\gamma$ and LPS were omitted. After a further $24 \mathrm{~h}$ incubation, plates were centrifuged, supernatants were harvested, and cells were subjected to the MTT assay (section 2.4). Cytokines were measured from supernatants by ELISA (section 2.6.1), and nitric oxide (NO) was measured from supernatants by Griess reaction (section 2.6.2).

\subsubsection{Single cell splenocyte suspension preparation}

Following euthanasia by $\mathrm{CO}_{2}$ asphyxiation, animals and surgical tools were rinsed in $70 \%$ ethanol before organ removal. Removed spleens were placed in CTCM and kept on ice until processing into single cell splenocyte suspensions. For this, organs were mashed through a $70 \mu \mathrm{M}$ strainer (BD Biosciences) placed on top of a $50 \mathrm{~mL}$ conical tube (Falcon) 
using the plunger from a $1 \mathrm{~mL}$ syringe (BD Bioscience). Strainers were rinsed with CTCM into the same tube, and tubes were then centrifuged to pellet cells. After discarding supernatants and loosening pellets, $2 \mathrm{~mL}$ RBC lysis buffer (Sigma-Aldrich) was added to lyse red blood cells. After two minutes incubation at room temperature, $8 \mathrm{~mL}$ CTCM was added to quench lysis. These splenocytes were washed via centrifugation, resuspended in 8mL CTCM and counted before use.

\subsubsection{Helper T cell activation: ConA-stimulated splenocytes}

$1 \times 10^{6}$ splenocytes (as prepared in 2.3.5) were seeded into wells of a flat-bottomed 96-well plate (Falcon) along with test compounds and $1 \mu \mathrm{g} / \mathrm{mL}$ ConA (Sigma-Aldrich) to stimulate cells, working to a final volume of $200 \mu \mathrm{L}$ per well. ConA was omitted for an unstimulated condition. After $48 \mathrm{~h}$ incubation (unless otherwise stated), plates were centrifuged, supernatants were carefully harvested, and cells were subjected to MTT assay (section 2.4).

\subsubsection{B cell activity: LPS-stimulated splenocytes}

$1 \times 10^{6}$ splenocytes (as prepared in 2.3.5) were seeded into wells of a flat-bottomed 96-well plate (Falcon) along with test compounds and $100 \mathrm{ng} / \mathrm{mL}$ LPS (E. coli, Sigma-Aldrich) to stimulate cells, working to a final volume of $200 \mu \mathrm{L}$ per well. LPS was omitted for an unstimulated condition. After $48 \mathrm{~h}$ incubation (unless otherwise stated), plates were centrifuged, supernatants were carefully harvested, and cells were subjected to MTT assay (section 2.4).

\subsection{MTT for viability or proliferation}

At the end of various assays, after supernatants were harvested, MTT Solution (see appendix 9.1) was added to cells for a final concentration of $1 \mathrm{mg} / \mathrm{mL}$ in $100 \mu \mathrm{L}$ CTCM. After a 45-minute incubation for RAW 264.7 cells, or 90 minutes for other cell types, cells were solubilised overnight using $100 \mu \mathrm{L}$ MTT Solubiliser (see appendix 9.1). The purple formazan that live, active cells produced from reducing MTT was measured on an EnSpire plate reader (Perkin Elmer, MA, USA) at $560 \mathrm{~nm}$. Data from this assay were used to glean compound toxicity data by comparing treated cells to vehicle controls, or proliferation by comparing stimulated and unstimulated cells. 


\subsection{Flow cytometry-based assays}

\subsubsection{General flow cytometry}

The BD FACS Canto II was used for all FACS-based analyses. The machine was calibrated using CS\&T beads that assess machine performance prior to collecting data. FACS Diva software collected the data, and FlowJo software (FlowJo LLC, OR, USA) was used for data analysis. Most data were collected using a High Throughput Sampler (HTS), which uses BD Sheath Solution with Surfactant to collect samples from round-bottom 96-well plates. Some samples were run in $5 \mathrm{~mL}$ polystyrene tubes and were collected using BD FACSFlow. Equipment, software and reagents mentioned in this paragraph are manufactured by BD Biosciences (CA, USA) unless stated otherwise.

Experiments followed a similar workflow. Unstained cells, single-stained cells, and stained CompBeads were used to adjust PMT voltages for optimal signal from samples. As samples were run, gates were drawn around populations of interest which were used to select 'stopping gates', defining the minimum recorded events per sample. Each time a new antibody clone was used, isotype controls were used to evaluate the non-specific binding of the marker to cells. Compensation was performed using single-stained cells, where possible, or was done with single-stained CompBeads. Compensation was calculated in FACS Diva prior to sample acquisition and was re-calculated in FlowJo before data analysis.

\subsubsection{Extracellular staining for flow cytometry}

Upon the conclusion of experiments, cells were washed and resuspended in FACS buffer (see appendix 9.1) for staining. In general, Fc receptors were blocked for 20 minutes on ice with 2.4G2 (anti-CD16, anti-CD32) antibodies. Fluorescently-labelled antibodies were added, and samples were incubated for 20 - 30 minutes on ice in the dark to label surface markers. This was followed with two washes in FACS Buffer via centrifugation at $300 \times \mathrm{g}$ for 4 minutes. Cells were resuspended in FACS buffer and passed through a mesh filter to remove clumped cells. Further FACS buffer was added to adjust cell concentration allowing for $<1000$ events/sec during sample acquisition. 
Note that the degranulation assay (section 2.5.7) involved cell staining during mid-assay cell stimulation. Similarly, proliferation assays required staining cells with CFSE before plating cells to start the assay (section 2.5.4).

\subsubsection{Zombie dye exclusion for assessing cell viability}

At the end of experiments, cells were washed in PBS and resuspended in PBS with 0.1\% (v/v) Zombie dye, NIR variant (BioLegend). Cells were incubated for 15 minutes in the dark, at room temperature, before further use. This fluorescent dye is membrane impermeable and only enters dead or dying cells.

\subsubsection{CFSE staining for assessing splenocyte proliferation}

$1 \times 10^{6}-1 \times 10^{7}$ cells were washed and resuspended in dPBS before incubating with $2.5 \mu \mathrm{M}$ CFSE for 15 minutes at $37^{\circ} \mathrm{C}, 5 \% \mathrm{CO}_{2}$. This staining was quenched with excess foetal calf serum, and cells were washed twice in dPBS before use. Cells were then stimulated with ConA or LPS, as in sections 2.3.6 and 2.3.7 respectively, though were subjected to flow cytometry analysis rather than MTT assays.

\subsubsection{Assessing macrophage phagocytosis using pHrodo bioparticles}

The PHrodo bioparticles used in this thesis are E. coli particles conjugated to a rhodopsinbased dye (Thermo Fisher). The particles become fluorescent within the low $\mathrm{pH}$ environment of a macrophage phagolysosome, with an Ex/Em of 560/585 nm. For use, lyophilised pHrodo bioparticles were suspended in the saline-based Live Cell Imaging Solution (see appendix 9.1) to a concentration of $1 \mathrm{mg} / \mathrm{mL}$ and placed in a sonicator bath for two minutes to break up aggregated particles. Aliquots were kept at $-20^{\circ} \mathrm{C}$ for long term storage.

For assays, RAW264.7 cells were seeded into 96-well plates at 100,000 cells/well and pre-treated with compounds, working to a volume of $140 \mu \mathrm{L}$. Cells were incubated for four hours at $37^{\circ} \mathrm{C}, 5 \% \mathrm{CO}_{2}$. PHrodo bioparticles were then added to wells for a final concentration of $25 \mu \mathrm{g} / \mathrm{mL}$ (unless otherwise stated) with further compound to maintain treatment concentration, working to a final volume of $200 \mu \mathrm{L} /$ well. After another fourhour incubation to allow for phagocytosis to occur (unless otherwise stated), plates were 
centrifuged before harvesting supernatants. Cells were washed twice with PBS to remove free pHrodo particles before resuspending in FACS Buffer for analysis by flow cytometry.

\subsubsection{Effector CTL generation}

OT-1 transgenic mice have a $\mathrm{T}$ cell repertoire that recognises SIINFEKL peptide (OVA 257-264) loaded in MHC Class I. Spleens from OT-1 mice were processed as in section 2.3.5, and splenocytes were incubated in T75 flasks or $150 \mathrm{~mm}$ sterile petri dishes at a concentration of $4 \times 10^{5}$ cells $/ \mathrm{mL}$ with $10 \mathrm{nM}$ SIINFEKL in $30 \mathrm{~mL}$ CTCM. This splenocyte preparation consists largely of naïve cytotoxic T lymphocytes (CTL), and B cells which are used here to present SIINFEKL and induce CTL activation.

After two days incubation, the now-primed effector cytotoxic T lymphocytes (eCTL) and B cells were harvested, pelleted via centrifugation, and resuspended in fresh CTCM to rest cells overnight. This rest period sets the eCTLs into a poised but inactive state, ready to use in the degranulation assay (section 2.5.7). B cells persist during this process to later be loaded with additional SIINFEKL to act as target cells during the degranulation assay.

\subsubsection{Assessing CTL degranulation using CD107a}

Following eCTL generation (section 2.5.6), the mixture of eCTLs and B cells were seeded into 96-well plates at $1.4 \times 10^{5}$ cells/well and cells were treated with compounds, working to final concentration of $140 \mu \mathrm{L}$. Cells were then incubated for four hours. To initiate degranulation, SIINFEKL peptide was added at a final concentration of $25 \mathrm{nM}$ to load target cells, along with $1 \mu \mathrm{g} / \mathrm{mL}$ CD107a:APC antibody (BD Biosciences) to label degranulatory activity on eCTLs. Further compound was also added at this time to maintain treatment concentration (final volume $200 \mu \mathrm{L}$ ). After a two-hour incubation, plates were centrifuged, supernatants were harvested, and cells were resuspended in FACS Buffer for analysis by flow cytometry. 


\subsection{Supernatant Analyses}

\subsubsection{ELISA}

A sandwich ELISA format was used to measure cytokines from assay supernatants, and a list of reagents and standard curve ranges can be found in appendix section 9.2.2. To perform ELISAs, wells in a 96-well MaxiSorp plate (Nunc, via Thermo Fisher) were coated with $50 \mu \mathrm{L}$ capture antibody solution overnight at $4^{\circ} \mathrm{C}$. Liquid was discarded, followed by the addition of $100 \mu \mathrm{L}$ 5\% FCS/PBS (blocking solution) to occupy any uncoated binding sites in wells. After two hours, the blocking solution was removed, and the plates were washed three times in ELISA wash solution (0.05\% Tween20 in PBS). Sample supernatants $(50 \mu \mathrm{L})$ were loaded into the plate alongside serially diluted recombinant target protein (target cytokine or IgM) to generate a standard curve. After a two-hour incubation at room temperature, or overnight at $4^{\circ} \mathrm{C}$, plates were washed four times with ELISA wash solution and $50 \mu \mathrm{L}$ of biotinylated detection antibody was added to wells for one hour at room temperature. Plates were washed six times in ELISA wash solution before adding $50 \mu \mathrm{L}$ streptavidin-conjugated horseradish peroxidase (SA-HRP) to wells. After a one-hour incubation at room temperature in dark conditions, plates were washed eight times with ELISA wash solution. TMB (100 $\mu \mathrm{L}$; see appendix 9.1) was added to wells and incubated for 5 - 15 minutes for a blue colour to develop. This reaction was 'stopped' with $100 \mu \mathrm{L} 0.18 \mathrm{M} \mathrm{H}_{2} \mathrm{SO}_{4}$ which turns the blue product to yellow, with colour intensity being proportional to the amount of target protein present. This yellow colour was measured using an EnSpire plate reader (PerkinElmer) at $460 \mathrm{~nm}$. After blank correction, the standard curve was used to interpolate analyte concentrations from sample absorbance values.

\subsubsection{Griess reaction}

Nitrates were measured from supernatants to assess NO production by macrophages. For this, $50 \mu \mathrm{L}$ of supernatants were transferred to wells of a flat-bottomed 96-well plate. On the same plate a standard curve was generated by making two-fold dilutions of $\mathrm{NaNO}_{3}$ from 500 - $0.488 \mu \mathrm{M}$ in CTCM; a blank was also included. To all wells, $50 \mu \mathrm{L}$ of Griess reagent (see appendix 9.1) was added. This reagent reacts with nitrite in the supernatant to form a purple colour. Absorbance at $570 \mathrm{~nm}$ was measured using an EnSpire plate reader (PerkinElmer). Sample NO concentrations were interpolated from standard curve data after blank correction. 


\subsection{Motility assays}

\subsubsection{Scratch assay}

A 12-well plate was seeded with 500,000 RAW 264.7 cells/well. After allowing cells to settle and adhere for four hours, $100 \mu \mathrm{L}$ micropipette tips were used to create a scratch through the cell monolayer. The scratches were inspected via microscopy, and images were taken to assess original scratch size; dots were drawn on the plate lid to identify the location along the scratch where images were taken. Treatments were added at this time, and cells were incubated. Further images were captured at 4, 8, and $24 \mathrm{~h}$ to measure the scratch and assess cell movement into the vacant area.

\subsubsection{ORIS wound closure assay for motility}

ORIS plates (Platypus Technologies, WI, USA) are 96-well plates with a silicon insert set in each well. After seeding with cells, the plug is removed and a $2 \mathrm{~mm}$ diameter cell-free exclusion zone (wound) is created in the centre of each well. To assess cell motility, 120,000 BMDM were seeded into wells of the ORIS plate in $100 \mu \mathrm{L}$ CTCM. Cells were incubated overnight to settle before plugs were removed with the supplied tool. Media was carefully aspirated and replaced with fresh media. Wells were then inspected via microscope, noting wells with disturbed exclusion zones: these wells were not used. Images of wells were also captured at this time to measure original wound size. Treatments were added to wells, and plates were incubated for three days (unless otherwise stated) before further images of each well were taken to measure final wound size. Upon the conclusion of experiment, plates were centrifuged, media was aspirated, and cell viability was assessed via MTT assay (section 2.4). ImageJ running the MRI Wound Healing Tool macro (Montpellier RIO Imaging, Montpellier, France) was used to measure wound size from images using the following script parameters: Method: variance; variance filter radius: 5; threshold: 50; radius open: 1; min. size: 10,000. Compound-induced changes to wound closure, representing motility, was assessed using the following equations:

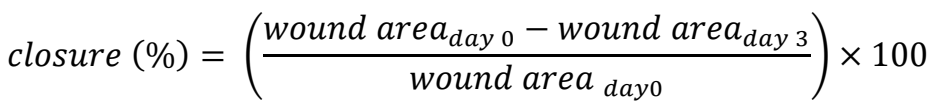

$$
\begin{aligned}
& \text { motility }(\text { as } \% \text { vehicle })=\left(\frac{\text { closure }_{\text {test compound }}}{\text { closure }_{\text {vehicle }}}\right) \times 100
\end{aligned}
$$




\section{Chapter 3: \\ Optimising assays to detect immunomodulation}




\subsection{Introduction}

A phenotypic drug screen is built with cell-based assays that collect data which assesses changes to cells and/or their activities in the presence of test compound. This cell-based approach suits the search for novel immunomodulators (including immunotoxic compounds) as the immune system is complex and there are many potential targets a drug could interact with to induce changes in the immune system. A high-content phenotypic drug screen should offer the opportunity to observe a test compound's effects, minimising falsely identifying a compound as having no immunomodulatory activity.

Six key immune cell processes were chosen to be represented in the screen to ensure compounds that alter the immune system would be identified. Phagocytes (macrophages/monocytes and neutrophils), granulocytes (neutrophils, basophils and eosinophils), helper T cells, cytotoxic T cells (CTL), and B cells were identified as cells of interest. These cells represent different components of the immune system that each play a significant role in the immune response, and their dysregulated activities can result in disease. The cellular processes these cells use to exert their function include antibody and/or cytokine production, phagocytosis, cell motility and degranulation.

In vitro assays are a convenient method to initially investigate test compounds due to their higher throughput format in comparison to in vivo assays, and this format uses smaller amounts of compound, however it has limitations. Not all cells are easily cultured or are easily sourced as primary cells, and granulocytes are a good example of this. Neutrophils have a central role in establishing inflammation and controlling pathogens through phagocytosis, but die quickly outside of the body[86]. With this constraint in mind, the cellular processes of the immune system were represented using cells more amenable to the in vitro environment. Macrophages share activities with neutrophils, and have well-established culturing methods, so were chosen for use to test compounds against these activities. Similarly, the other granulocytes can be difficult to maintain outside the body, however their degranulatory activities are shared with CTL which can be used to assess the modulation of degranulation by test compounds[87-89].

The practicality of the work should also be considered when choosing the assaying methods for the screening strategy. Assays need to be medium-throughput and costs-effective to 
facilitate the testing of a large number of compounds. For these reasons, assays that can be performed in a 96-well plate format and use cheaper, and/or fewer reagents are preferred. During the assay optimisation process, assays should be fine-tuned to ensure immunomodulation can be detected, that is, both increases and decreases to assay outputs (i.e. cell activities) can be observed. Exploring the range of outputs that each assay can provide may result in the ability to capture more information about compound activity during testing.

With these considerations in mind, assays that measure macrophage activation, phagocytosis and motility were optimised for drug discovery. Assays for helper T cell activation, cytotoxic T lymphocyte degranulation and B cell activity were also optimised.

\subsection{Aims}

This chapter describes the selection and optimisation of assays that detect immunomodulatory activity. The objectives of this chapter are to:

1. Develop and/or explore assay designs that measure immune cell activities

2. Optimise assays for drug discovery, including simplifying assay design, enhancing throughput, and increasing outputs from assays, where possible

3. Ensure assays are appropriately sensitive to detect changes to cell activity that are induced by compounds 


\subsection{Results}

\subsubsection{Macrophage Activation}

Because macrophages are quick-responding innate immune cells that produce cytokines upon activation, they have central roles in shaping the immune response making them a promising target for immunomodulatory treatments. The cytokines and reactive oxygen and nitrogen species they produce upon activation contribute to pathogen control, the establishment of inflammation, and shaping the adaptive immune response. Compounds can alter macrophage activation which, in turn, can alter the general immune response.

A common protocol was used to assess the effects of test compounds on macrophage activation. Here, plated cells are incubated with IFN $\gamma$ to induce a poised, pro-inflammatory M1 macrophage. This priming step is followed by activation with lipopolysaccharide (LPS), a cell wall component of gram-negative bacteria and ligand of TLR-4, which results in the macrophage expressing bactericidal reactive nitrogen species, the pro-inflammatory cytokines IL-12, and TNFa, and regulatory IL-10, among others[90-92]. The macrophage perceives an infection and these activities attempt to limit the pathogen's persistence by destroying the threat, inducing inflammation, and eliciting and shaping the adaptive immune response. Decreases in these activities, by test compounds, suggests immunosuppressive effects, whilst specific decreases to TNF $\alpha$ and IL-12, and increases to IL-10 production suggests a compound has induced an anti-inflammatory M2 macrophage phenotype[33]. Changes to nitric oxide (NO) production by compounds indicates altered bactericidal activity.

\subsubsection{Optimisation}

Previously our group has primed cells with $20 \mathrm{U} / \mathrm{mL}$ IFN $\gamma$ for $18 \mathrm{~h}$, followed by a 24-hour stimulation with $200 \mathrm{ng} / \mathrm{mL}$ LPS to activate macrophages[93,94], and this resembles commonly used macrophage activation methods[92]. This design allows for the analysis of NO, IL-10, IL-12 and TNF $\alpha$ production by macrophages. To ensure the established IFN $\gamma$ and LPS concentrations were still appropriate to activate macrophages in vitro, and to ensure this activation was sensitive to modulation by drug, these two reagents were titrated against each other to find conditions where cytokines were at, or near, half-maximum 
production. This would allow for the detection of both increases and decreases to NO and cytokine production when macrophages are activated in the presence of test compound.

In the absence of LPS, NO production by macrophages increased with increasing concentrations of IFN $\gamma$. With LPS in the system, this effect was present but is less remarkable. Mid-range NO production was seen after priming cells with $20 \mathrm{U} / \mathrm{mL}$ IFN $\gamma$ across all LPS concentrations (Figure 3.1, A). TNF $\alpha$ production increased with higher LPS concentrations across all priming conditions, and priming with $20 \mathrm{U} / \mathrm{mL}$ IFN $\gamma$ produced mid-level TNF $\alpha$ production across all LPS concentrations (Figure 3.1, B). IL-12 production was unaffected by priming condition and between 20-200 ng/mL LPS generated mid-range levels of observed IL-12 production (Figure 3.1, C). IL-10 increased with increasing LPS concentrations and priming with $20 \mathrm{U} / \mathrm{mL}$ IFN $\gamma$ induced mid-range IL-10 production after a minimal stimulation with $2 \mathrm{ng} / \mathrm{mL}$ LPS (Figure 3.1, D). Across the four assay outputs, activating macrophages with $20 \mathrm{U} / \mathrm{mL}$ IFN $\gamma$ followed by $200 \mathrm{ng} / \mathrm{mL}$ LPS induced mid-range cytokine production that was detectable by ELISA. The established macrophage activation protocol used previously in our group suits the assessment of compound-mediated macrophage immunomodulation. 

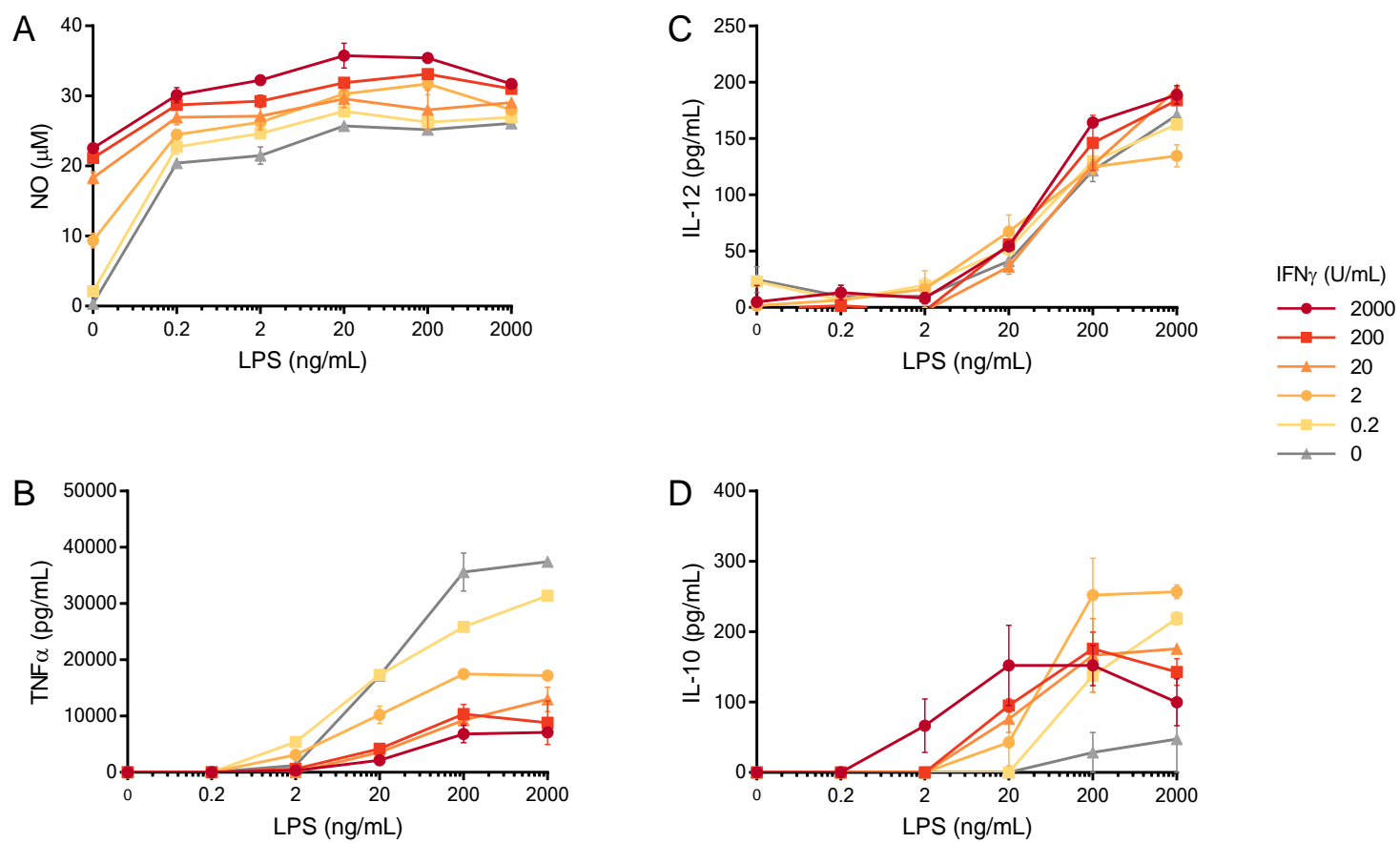

Figure 3.1. Priming macrophages with $20 \mathrm{U} / \mathrm{mL}$ IFN $\gamma$, and stimulating with $200 \mathrm{ng} / \mathrm{mL}$ LPS, induces mid-range NO and cytokine production.

RAW 264.7 cells were seeded at 50,000 cells/well and primed for $18 \mathrm{~h}$ with indicated concentrations of IFN $\gamma$. After a $24 \mathrm{~h}$ stimulation with LPS at indicated concentrations, supernatants were harvested. NO was measured by Griess reaction (A) and TNF $\alpha$ (B), IL-12 (C) and IL-10 (D) measured by sandwich ELISA. Data are means and SEM from one experiment conducted in duplicate.

An MTT assay was used to observe any cytotoxic effects of IFN $\gamma$ or LPS on RAW 264.7 cells. With the addition of LPS, the viability of activated RAW 264.7 dropped to 50\% when compared to unactivated cells (Figure 3.2, A). Because this did not appear to act in a concentration-dependent manner, factors other than cytotoxicity were considered. Perhaps the decrease in MTT was not due to cytotoxicity: MTT measures metabolic activity from which cytotoxicity is assumed when this activity decreases. A decrease can also be observed if cell numbers differ between conditions. RAW 264.7 cells have a doubling time of 18 hours, so it was hypothesised that activation with IFN $\gamma$ and LPS stops RAW 264.7 cell proliferation. With relatively fewer cells per well in activated conditions the MTTreducing capacity, i.e. metabolic activity, of these wells would be lower. 
Paclitaxel, an anti-proliferative, was used to test this hypothesis. Here, paclitaxel-treated cells were compared to vehicle-treated equivalents in both unactivated and activated states (Figure 3.2, B). With $1 \mu \mathrm{M}$ Paclitaxel, proliferating, unactivated RAW 264.7 cells have reduced metabolic activity compared to vehicle-treated cells. Activated cells retained the same metabolism as vehicle-treated cells with this same treatment. The lack of effect of paclitaxel on activated RAW 264.7 cells suggested they were no longer proliferating. The recovery of unactivated cell metabolism with $10 \mu \mathrm{M}$ Paclitaxel can be explained by the LPS-mimicry seen with this compound, where their activation caused an increase in metabolic activity[95]. Paclitaxel $(100 \mu \mathrm{M})$ is assumed to be cytotoxic due to the decreased cell metabolism in both cell states. These data supported the idea that activated macrophages stop proliferating. By including unactivated RAW 264.7 cells in this assay, compound effects on proliferation can also be assessed by comparing MTT data between the two cell states.
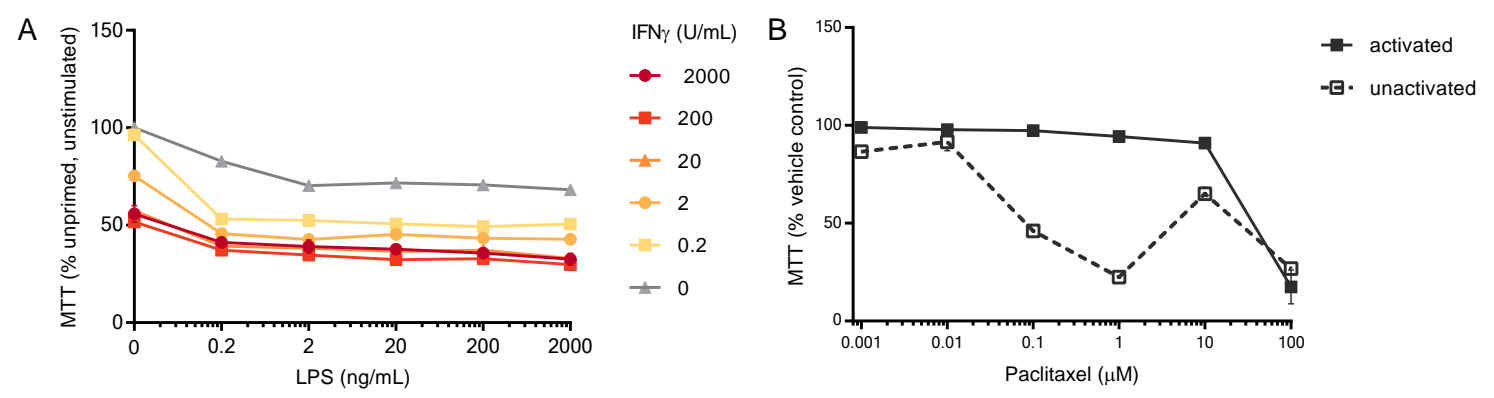

Figure 3.2. IFN $\gamma$ and/or LPS alters RAW 264.7 cell proliferation.

RAW264.7 cells were plated at 50,000 cells/well and primed with indicated concentrations of IFN $\gamma$ for $18 \mathrm{~h}$. LPS, at indicated concentrations, was then added to wells for $24 \mathrm{~h}$ before MTT was added to wells to assess cell activity compared to unactivated cells (A). 50, 000 RAW 264.7 cells were seeded in wells and incubated for $18 \mathrm{~h}$ with $20 \mathrm{U} / \mathrm{mL}$ IFN $\gamma$. Paclitaxel was added at indicated concentrations with $200 \mathrm{ng} / \mathrm{mL}$ LPS. Cells were then assessed for viability by MTT assay and compared to vehicle controls (B). Data are means and SEM from one experiment each, conducted in duplicate. 


\subsubsection{Altering macrophage activation with Risperidone}

Risperidone, an atypical antipsychotic, demonstrates anti-inflammatory activity[94,96]. Increased IL-10 and decreased IL-12 production has been observed in risperidone-treated, activated bone-marrow derived macrophages[94,97]. It was a suitable positive control to assess if the chosen assay design could detect immunomodulation. For this experiment, the concentrations of risperidone were chosen based upon the previous experiments[94]. Treatment with $50-200 \mu \mathrm{M}$ Risperidone, where viability is largely unaffected, slightly reduced pro-inflammatory TNF $\alpha$ levels whilst anti-inflammatory IL-10 levels increased. However pro-inflammatory IL-12 increased in these activated RAW264.7 cells (Figure 3.3).

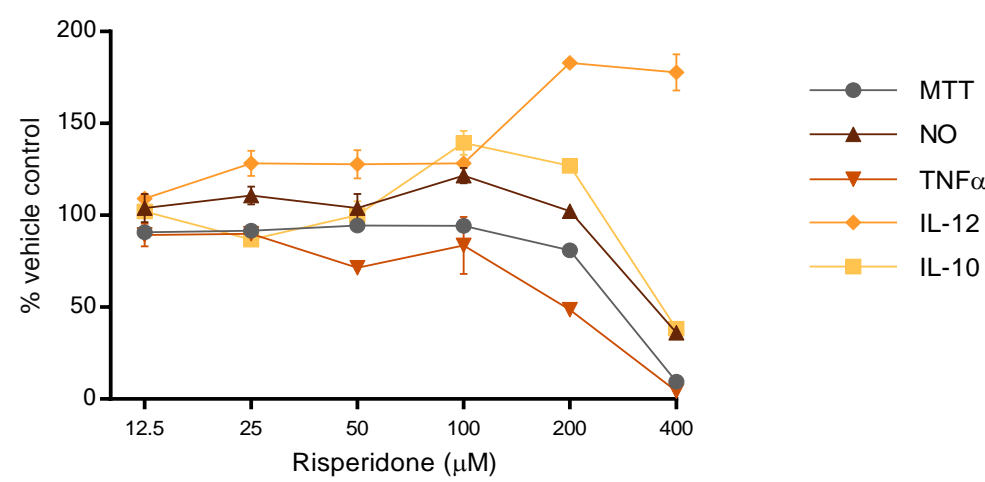

Figure 3.3. Risperidone alters cytokine production in activated RAW 264.7 cells.

50,000 RAW 264.7 cells were seeded into wells. After priming with $20 \mathrm{U} / \mathrm{mL}$ IFN $\gamma$ for $18 \mathrm{~h}$, indicated concentrations of Risperidone, or vehicle control, was added alongside $200 \mathrm{ng} / \mathrm{mL}$ LPS. After $24 \mathrm{~h}$, supernatants were analysed by ELISA and Griess reaction, and cell viability was assessed by MTT. Data are means and SEM from one experiment conducted in duplicate.

Because the activity of Risperidone-treated macrophages differed from independent observations, the experiment was repeated with a different RAW 264.7 cell stock. Data from this repeat (not shown) were similar, indicating an issue either with Risperidone, or the cells. This assay was then performed with BMDM in place of RAW264.7 cells to see if previous findings could be reproduced (Figure 3.4). Risperidone increased IL-10 production in BMDM, as seen with RAW264.7 cells, however BMDM production of TNF $\alpha$ was unaffected by Risperidone treatment, and IL-12 production decreased. These antiinflammatory effects agreed with previous findings and differed to the RAW 264.7 data. This suggested that RAW264.7 cells may not represent macrophage activity appropriately. 


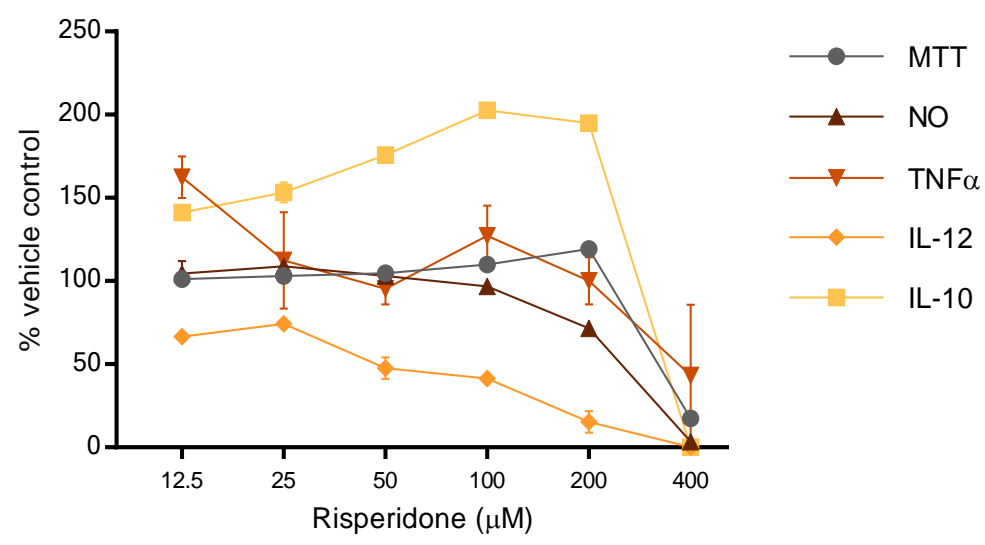

Figure 3.4. Risperidone shifts activated BMDM cytokine production to resemble an M2 phenotype. 100,000 BMDM were seeded into wells and primed with $20 \mathrm{U} / \mathrm{mL}$ IFN $\gamma$. Risperidone, or vehicle control, was added to wells at indicated concentrations and stimulated with $200 \mathrm{ng} / \mathrm{mL}$ LPS. After $24 \mathrm{~h}$, supernatants were analysed by ELISA for cytokines, and Griess for NO production. Cell viability was assessed by MTT. Data are means and SEM from one experiment conducted in duplicate.

\subsubsection{Summary}

An M1 macrophage phenotype can be elicited from RAW 264.7 cells after priming cells with $20 \mathrm{U} / \mathrm{mL}$ IFN $\gamma$ for 18 hours, followed by LPS stimulation for 24h. At the end of this activation, NO, TNF $\alpha$, IL-12 and IL-10 can be measured from supernatants. By treating macrophages with compound during LPS stimulation, changes to cytokine production can be assessed to identify immunomodulatory compound activity. Further work needs to be done to investigate how appropriate RAW 264.7 cells are for investigating a compound's activity on macrophage activation, though it is hypothesised that these cells can still detect the ability of test compounds to alter macrophage activity, but that the specific changes may not be representative of other macrophage populations.

By treating unactivated and activated RAW 264.7 cells, differences in MTT data can indicate a compound has anti-proliferative activity. The additional testing of compounds on unactivated RAW 264.7 cells during the macrophage activation assay provides more data about compound activity. 


\subsubsection{Assessing phagocytosis with pHrodo bioparticles}

Phagocytosis is the process through which macrophages, and other innate immune cells, take up large particles from their environment. During infection, macrophages phagocytose bacteria and other pathogens, which limits pathogen spread and facilitates antigen presentation to engage $\mathrm{T}$ cell help. Cell debris, generated after cells have undergone necrosis and apoptosis, is also cleared up by macrophages through phagocytosis. Impaired phagocytic activity can result in a susceptibility to infection and impaired healing and is implicated in the progression of SLE[78,98]. For these reasons there is interest in understanding a compound's ability to alter phagocytosis.

The uptake of polystyrene beads, latex beads, zyomsan, and bacteria have been used to determine the phagocytic activity of macrophages[99]. Known quantities of these particles are incubated with macrophages and, after allowing for phagocytosis to occur, either remaining extracellular particles or ingested particles are quantified. With the ability to fluorescently label these materials, internalised particles can be measured using microscopy or flow cytometry. PHrodo bioparticles are E. coli fragments that are conjugated to a proprietary dye that only produces a signal when exposed to the low $\mathrm{pH}$ conditions inside a phagolysosome. Only phagocytosed particles emit a fluorescent signal, and measuring this fluorescence determines phagocytic activity[100]. This eliminates the interfering false signal from fluorescent particles that have adhered to the outside of the cell. For these reasons, assessing phagocytosis with pHrodo bioparticles was chosen. Because these bioparticles come from $E$. coli, this should elicit cytokine production by macrophages. This was also explored during assay optimisation to provide more data on macrophage activity during this process. 


\subsubsection{Optimisation}

To optimize this assay, RAW264.7 cells were incubated with different concentrations of pHrodo for two or four hours before measuring fluorescence by flow cytometry (see appendix 9.3.1 for gating strategy). PHrodo bioparticles appeared to always be in excess, based on FSC vs SSC FACS data observed during these experiments. To conserve reagent and optimise assay sensitivity to immunomodulation, a concentration of $25 \mu \mathrm{g} / \mathrm{mL}$ pHrodo was selected. This concentration elicited a measurable level of fluorescence from phagocytosing cells (Figure 3.5, A) and half-maximum TNF $\alpha$ production after a 4-hour incubation; TNF $\alpha$ was not detectable at 2 hours (Figure 3.5, B). This pHrodo concentration is lower than the manufacturer's recommended concentration of $1000 \mu \mathrm{g} / \mathrm{mL}$ but is adequate for assessing phagocytic activity.
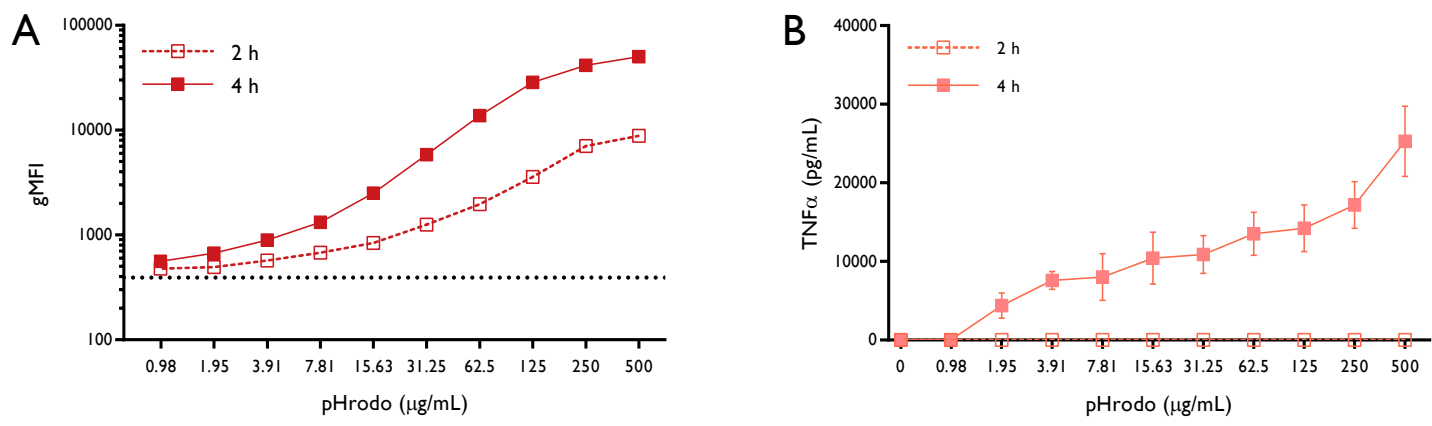

Figure 3.5. $4 \mathrm{~h}$ pHrodo incubation allows for TNFa measurement as well as phagocytosis assessment. 100,000 RAW 264.7 cells were seeded into a 96-well plate and incubated with indicated concentrations of pHrodo bioparticles. After $2 \mathrm{~h}$ (dashed) or $4 \mathrm{~h}$ (solid), cell fluorescence resulting from pHrodo phagocytosis was assessed by flow cytometry; dotted black line is background fluorescence. TNF $\alpha$ was measured from supernatants; TNF $\alpha$ was undetectable with a $2 \mathrm{~h}$ pHrodo incubation (B). $25 \mu \mathrm{g} / \mathrm{mL}$ pHrodo sees mid-range TNF $\alpha$ production, and detectable phagocytosis. Data are means and SEM from one experiment conducted in duplicate. 


\subsubsection{Inhibiting phagocytosis with Latrunculin A}

As phagocytosis involves cytoskeletal integrity to form a phagocytic cup around the target, the actin depolymeriser, Latrunculin A (LatA), is a suitable positive control for phagocytosis inhibition[101]; a wide concentration range of LatA was used to ensure this activity was captured. At concentrations of drug where cell viability was unaffected, $0.001-10 \mu \mathrm{M}$, phagocytosis was reduced to $50 \%$ or below that of vehicle-treated cells (Figure 3.6). TNF $\alpha$ production was reduced with $0.01-10 \mu \mathrm{M}$ Lat A. IL-6 production was also measured during this experiment and was altered by LatA treatment. IL-6 production increased with LatA concentrations below $1 \mu \mathrm{M}$ and decreased with higher concentrations. These data suggest the alteration of phagocytosis and concurrent cytokine production can be detected with this assay design.

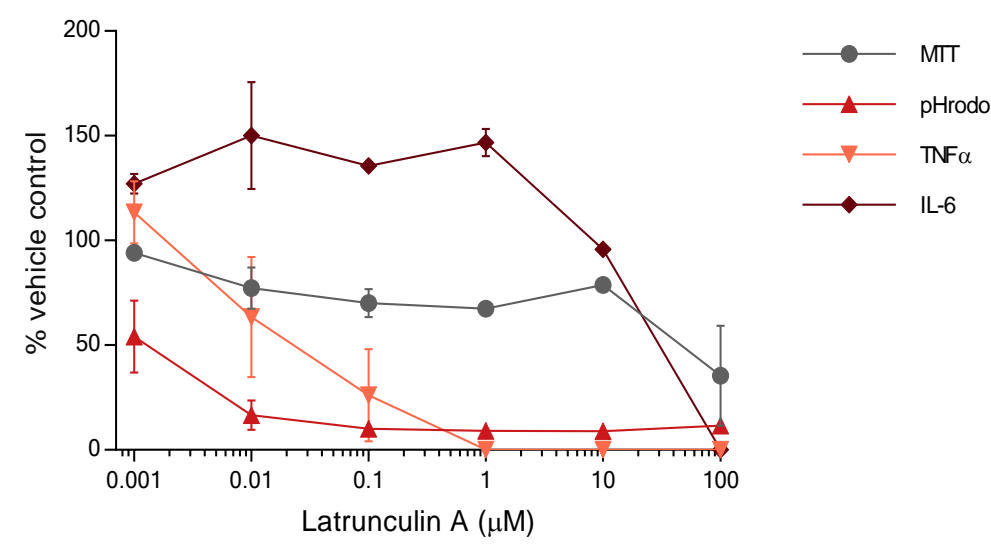

Figure 3.6. Latrunculin A inhibits phagocytosis.

100,000 RAW 264.7 cells were seeded into each well of two 96-well plates. LatA, or vehicle control, was added to wells at indicated concentrations for $4 \mathrm{~h}$. At this point, one plate was subjected to MTT assay for viability; pHrodo bioparticles were added to the second plate with further drug to maintain concentration. After $4 \mathrm{~h}$, cells from the latter plate were subjected to flow cytometry to measure fluorescence of phagocytosed pHrodo. Supernatants were analysed by ELISA for IL-6 and TNF $\alpha$. Data are means and SEM from one experiment conducted in duplicate.

\subsubsection{Summary}

After a four-hour pre-treatment with test compound, RAW 264.7 cells incubated with $25 \mu \mathrm{g} / \mathrm{mL}$ pHrodo bioparticles for four hours allows for the assessment of compoundinduced changes to phagocytosis and concurrent cytokine production. These cell processes can be inhibited by LatA, a suitable positive control for this assay. 


\subsubsection{Cell Motility}

All immune cells require a level of motility to move into various sites of the body. For example, innate immune cells, such as macrophages and dendritic cells, move through tissues to sites of infection, and between tissues and secondary lymphoid organs to interact with the adaptive immune system. Upon activation lymphocytes need to mobilise from secondary lymphoid organs and enter the blood stream to circulate to sites of infection. Altering this process can impair the immune response. For inflammatory diseases, impairing motility can result in reduced inflammation as cells cannot move throughout the body and carry out their aberrant functions. This is, in part, how colchicine exerts its antiinflammatory effects in the treatment of gout[67]. An assay to measure changes to cell motility by compound treatment was optimised to identify compounds that can alter cell motility.

\subsubsection{Optimisation}

The popular scratch assay was investigated as a method to assess cell motility. Here, a 'scratch' is made through a monolayer of cells, creating a clear zone flanked by cells. Over time, motile cells will enter this zone, filling in the scratch. Changes to the scratch is assessed under the microscope and indicates the cells' capacity to move[102]. Comparing the ability of compound-treated cells to vehicle-treated cells can identify the ability of the compound to alter this process. Macrophage motility requires heavy involvement of cytoskeletal remodelling, especially of microfilaments. These microfilaments extend filopodia against which the cell moves forward[103]. By disrupting the polymerisation of actin, which forms these microfilaments, the motility of macrophages is inhibited[104,105]. For this reason, LatA was used as a positive control for altering cell motility.

After identifying an appropriate seeding density of 500,000 cells per well to achieve a monolayer of RAW 264.7 cells in a 12-well plate, a scratch was made through the monolayer to create an area for cells to move into (Figure 3.7). Cells were then treated with a range of LatA concentrations, and a vehicle control. Scratches were inspected after two, four, and 24-hours to determine a suitable timeframe to allow for cell movement to occur. Over time, it was observed that the proliferation of RAW 264.7 cells interfered with the motility assessment. These semi-adherent cells were not inclined to remain within a 
monolayer and appeared to drift into the cell free zone, and increased cell numbers also depleted media before any motility was observed. Additionally, this scratch assay was not medium throughput, and generating reproducible scratches was difficult. There also was concern that creating the scratch would damage cells, activating the remaining macrophages via damage signals which could interfere with cell motility[103,106,107]. This assay format was abandoned and other formats to assess motility were investigated.
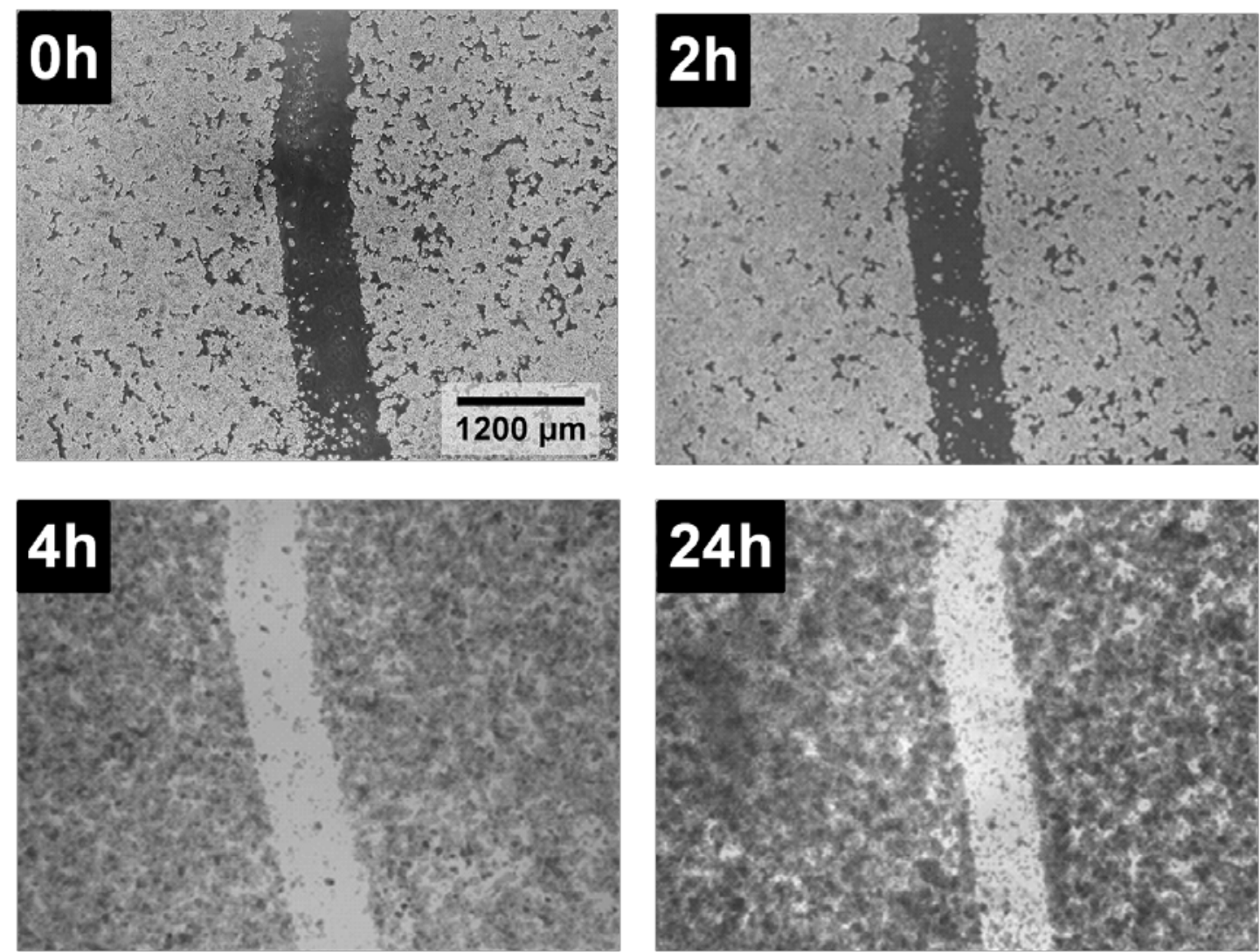

Figure 3.7. RAW264.7 proliferation interferes with motility assessment.

RAW264.7 cells were seeded at 500,000 cells per well in a 12-well plate. Scratches were made using a 100 $\mu \mathrm{L}$ pipette tip. Plates were incubated, with photographs taken at times indicated on images.

With an appreciation from the scratch assay that macrophages were slow to move (requiring more than a day), and that proliferation (where cell cycle takes less than a day) interfered with motility assessment, non-proliferating (BMDM) were used instead. With these cells, any changes to wound size would be due to cells moving into the space without wound size changing due to increasing cell density. Also, a medium-throughput format of a wound closure assay was selected for use: the ORIS wound closure assay consists of 96-well plates 
with pre-inserted silicon plugs in each well. After seeding wells and allowing cells to adhere to the plate, plugs are removed leaving a $2 \mathrm{~mm}$ diameter cell-free zone, or 'wound', in the centre of each well. Over time, cells move into the free space, decreasing wound size. A reduction of wound size over time indicates cells are motile. These changes solved many of the identified problems, including the reproducibility of cell-free zones, limiting cell damage, improving throughput and removing the interference of motility assessment due to cell proliferation.

The first step in optimising the ORIS-based motility assay protocol was to determine a seeding concentration that resulted in a confluent layer of cells, and to determine how long cells needed to fill in the empty space. At least 120,000 cells/well were required to produce a monolayer of cells, which also made for easy image analysis. The wound never fully closed during this experiment, and there was no effect of seeding density on motility rate (Figure 3.8). By four days the media required changing so it was decided that a three-day incubation was sufficient: cell motility can be observed within this timeframe. To reduce the number of cells required to complete the assay, a seeding density of 120,000 cells per well was selected.

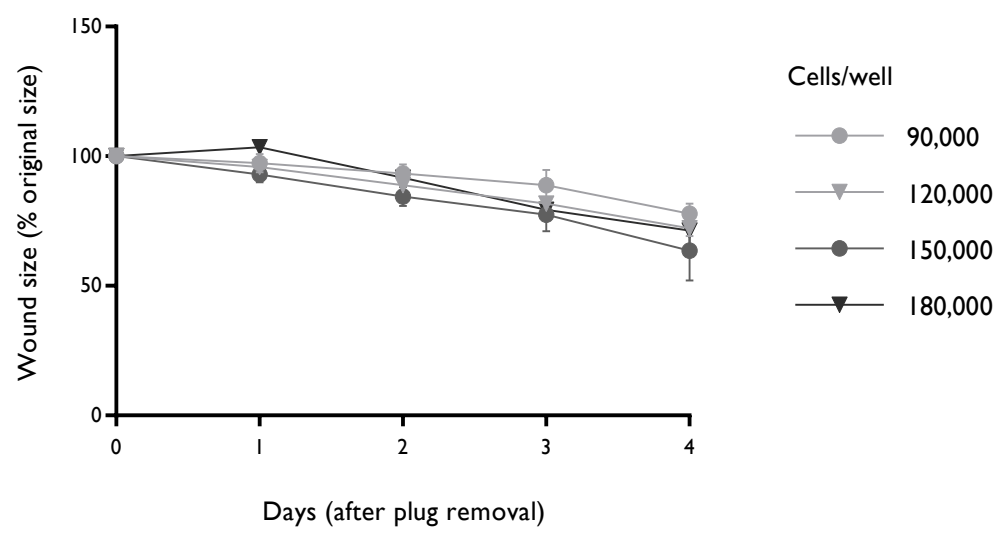

Figure 3.8. Seeding density does not affect BMDM motility.

Wells in an ORIS plate were seeded with indicated numbers of BMDM. After allowing cells to settle overnight, plugs were removed and wound size at the time points indicated were measured using ImageJ. Data are means and SEM from one experiment conducted in duplicate. 


\subsubsection{Latrunculin A inhibits macrophage motility}

LatA was used as a positive control for altering cell motility, and a wide concentration range was used to ensure this inhibitory activity was observed. Wound closure was assessed by comparing the change in wound size from day 0 to day 3 , and this change was expressed as a percentage of original wound size. This was done for individual wells to account for any differences between original wound size. Changes in wound size were then compared between compound and vehicle treatments. This was done using the following equations.

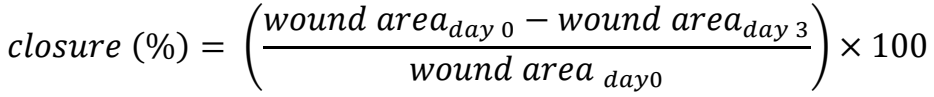

$$
\begin{aligned}
& \text { motility }(\text { as } \% \text { vehicle })=\left(\frac{\text { closure }_{\text {test compound }}}{\text { closure }_{\text {vehicle }}}\right) \times 100
\end{aligned}
$$

LatA successfully inhibited cell motility at all concentrations tested, although cell viability was altered with Lat A treatment (Figure 3.9). However, with $0.01 \mu \mathrm{M}$ Lat A, where viability is $93 \%$ of vehicle control, motility is only $12 \%$ compared to vehicle. That is, cells treated with $0.01 \mu \mathrm{M}$ LatA only closed the wound by roughly one-tenth of what vehicletreated cells achieved. Detecting this expected decrease indicates that changes to cell motility can be assessed using this assay design. 


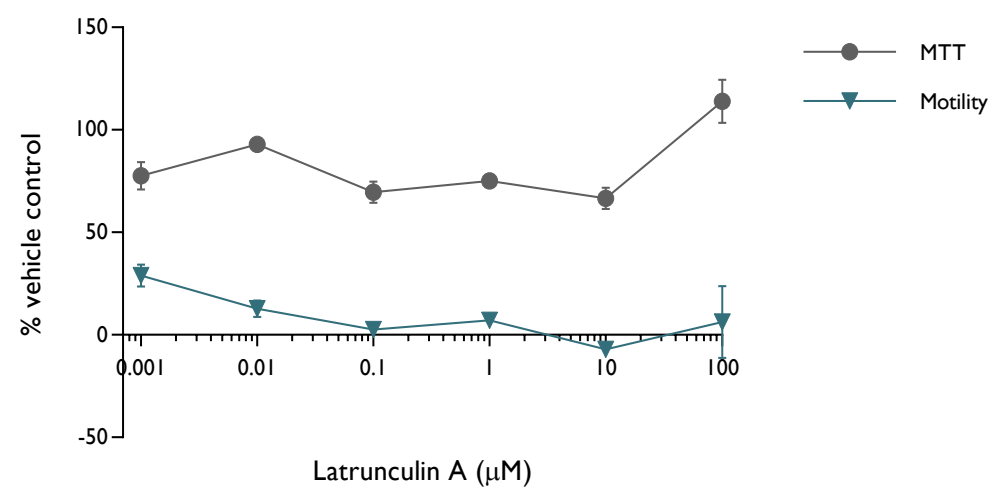

Figure 3.9. Inhibition of motility using LatA is measurable using the ORIS assay.

120,000 BMDM were seeded into wells of an ORIS plate. The following day after cells had settled, plugs were removed and LatA, or vehicle control, was added at indicated concentrations. After observing cell migration for three days, cells were subjected to MTT assay to assess viability. Closure in vehicle-treated wells averaged 20.39\% (SD = 3.46\%). Data are means and SEM from one experiment conducted in duplicate.

\subsubsection{Summary}

Wound closure is used as a measure of cell motility. 120,000 BMDM were seeded into wells of an ORIS wound closure plate. After allowing cells to adhere overnight, plugs are carefully removed, and media aspirated. After adding fresh media, photos were taken for original wound size measurement. Treatments were added, and cells incubated for three days before taking photographs to measure endpoint wound size. Cells were then subjected to MTT for viability, and photographs analysed by ImageJ running MRI wound closure script to assess wound closure. LatA is a suitable positive control for alteration of cell motility. 


\subsubsection{Assessing helper $\mathrm{T}$ cell activity using ConA}

Helper T cells (Th cells) can be considered coordinators of immune responses. When active, these cells produce cytokines which modify the greater immune response to promote activities that best combat the pathogen at hand. However, dysregulated Th cell activity is implicated in several autoimmune and inflammatory diseases because of their support of inappropriate immune responses. Influencing the differentiation of T cells into favourable subsets during immune dysfunction is an avenue for disease treatment because of their co-ordinating role in the immune system. In other words, altering Th cell activity can provide control over many immune processes. Glatiramer acetate alters Th cell differentiation and promotes anti-inflammatory Treg activity in vivo, helping sufferers of Multiple sclerosis by indirectly suppressing the pro-inflammatory activities of innate immune cells and CTL[108].

Different stimuli have been used to activate T cells in vitro. Anti-CD3/CD28 antibodies target the TCR to activate cells, but the cheaper ConA (Concanavalin A) achieves the same goal. ConA is a plant cell lectin that binds the T cell receptor (TCR) which results in T cell activation and the production of cytokines[109]. Upon activation, Th cells differentiate into one of four main subsets with each subset producing a distinct repertoire of cytokines. By measuring four key cytokines, the subset bias of active Th cells can be inferred. Th1 cells produce IFN $\gamma$, a pro-inflammatory cytokine that promotes activity against intracellular pathogens. Th2 cells produce anti-inflammatory IL-4, supporting humoral responses that deal with pathogens in the circulation and in airways. IL-17 is produced by Th17 cells, suggested to enhance neutrophil activity, and to promote mucosal immunity and inflammation. The last subset is made of Tregs, which produce IL-10 to regulate the immune response by dampening many immune cell activities.

\subsubsection{Optimisation}

Different concentrations of ConA were titrated against splenocytes to activate Th cells. After $48 \mathrm{~h}$ or $72 \mathrm{~h}$, supernatants were analysed by ELISA for IFN $\gamma$, IL-10, IL-4 and IL-17A. The aim of this experiment was to find an appropriate concentration of ConA that elicits mid-range production of all four key cytokines from $\mathrm{T}$ cells as this would allow the 
detection of reduced or additional cytokine production when $\mathrm{T}$ cells are treated with compounds.

Generally, cytokine production increased with ConA concentrations, though within the shorter timeframe at least $0.25 \mu \mathrm{g} / \mathrm{mL}$ ConA was needed to detect IFN $\gamma$, IL10 and IL-17A (Figure 3.10). At $72 \mathrm{~h}$, greater concentrations of cytokines were detected in supernatants, except for IL-4 (Figure $3.10 \mathrm{~B}$ ). Stimulation with $1 \mu \mathrm{g} / \mathrm{mL}$ ConA elicit half-maximum cytokine production of IFN $\gamma$, IL-10 and IL-17A (Figure 3.10, A, C, D). Although IL-4 production with $1 \mu \mathrm{g} / \mathrm{mL}$ ConA appears to be low, increasing ConA would cause IL-10 production to be near maximum production where increase of this cytokine by test compounds may not be detectable. A 48-hour stimulation with $1 \mu \mathrm{g} / \mathrm{mL}$ ConA results in easily detected, mid-range levels of IFN $\gamma$, IL-4, IL-17A and IL-10, and therefore was selected to activate helper $\mathrm{T}$ cells.
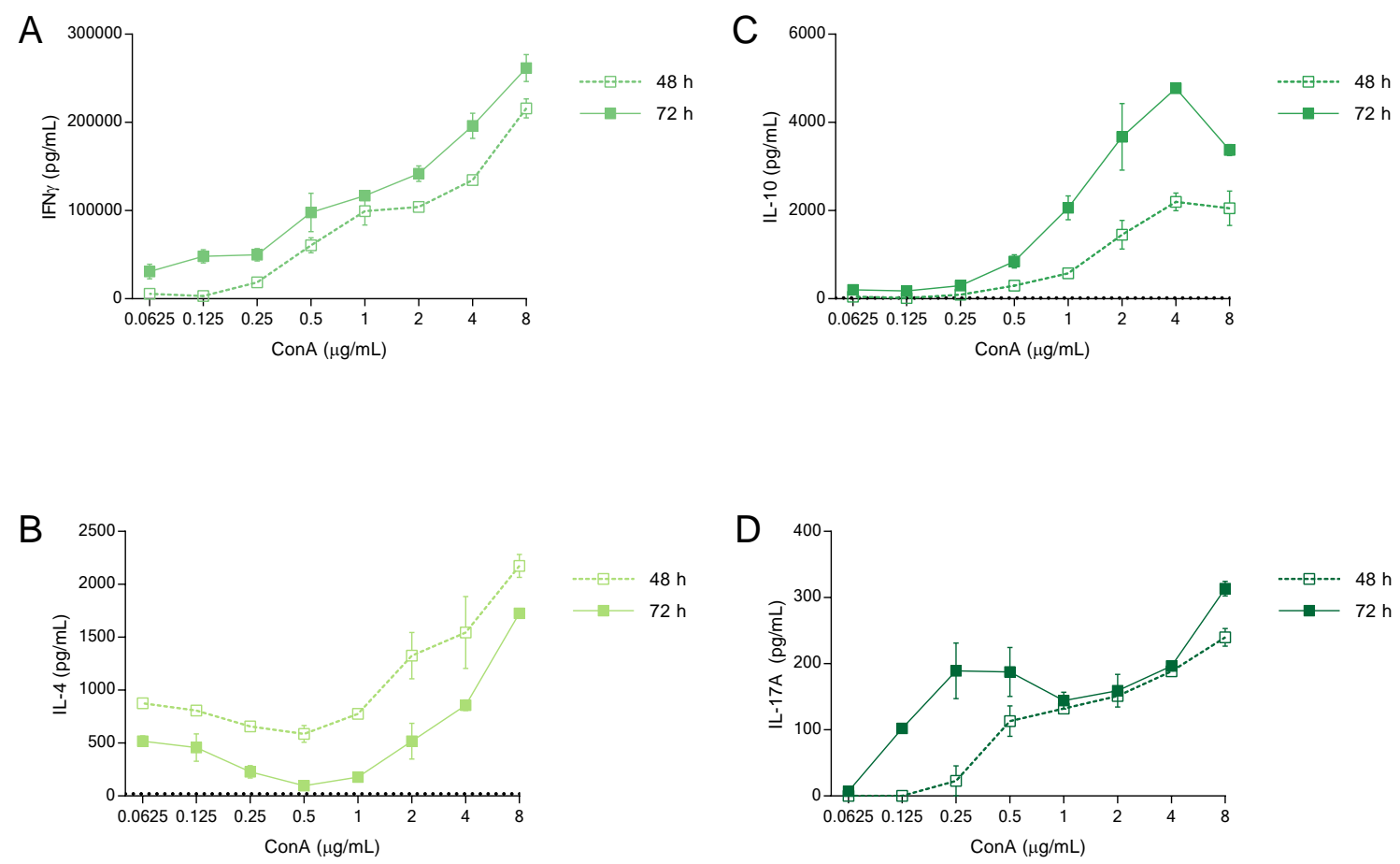

Figure 3.10. ConA stimulation of splenocytes induces helper $T$ cell differentiation into four Th subsets. $1 \times 10^{6}$ splenocytes were seeded into wells with indicated concentrations of ConA. After $48 \mathrm{~h}$ (dashed) or $72 \mathrm{~h}$ (solid), supernatants were harvested, and levels of IFN $\gamma$ (A), IL-4 (B), IL-10 (C) and IL-17(D) were measured by ELISA. $1 \mu \mathrm{g} / \mathrm{mL}$ ConA stimulates $\mathrm{T}$ cells to produce mid-range levels of all four cytokines. Data are means and SEM from one experiment, conducted in duplicate. 
As the cells used in this assay came from a mixed-cell splenocyte culture, made largely of B cells and T cells, and acknowledging that activated Th cells can in turn activate B cells via CD40L, IgM production by B cells during the ConA stimulation was measured to assess indirect B cell activation[110,111]. If activated, B cells could produce the same cytokines as Th cells which would affect data interpretation, and the scope of the assay. IgM levels were measured from cell supernatants from the last experiment as an indicator of B cell engagement in this assay (Figure 3.11). IgM levels did not differ significantly between the 48-hour ConA stimulation and unstimulated conditions, whereas T cells stimulated for $72 \mathrm{~h}$ resulted in B cells also being activated. This explains the decreased IL-4 seen in the previous experiment as IL-4 is taken up by active B cells. This finding confirms that the 48-hour stimulation duration is best as it isolates Th cell activity from B cell activity.

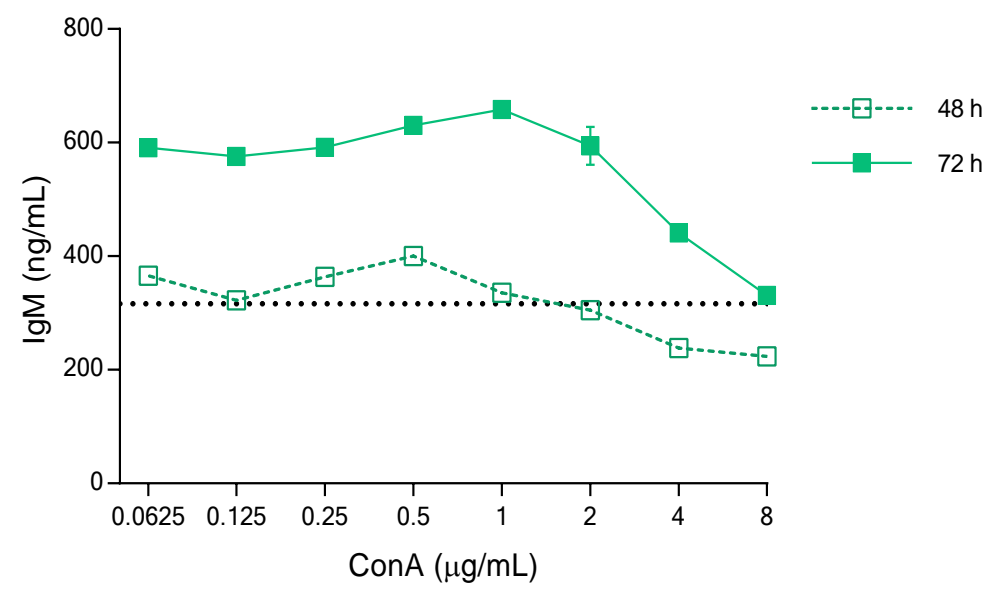

Figure 3.11. Splenocytes stimulated with ConA for $72 \mathrm{~h}$ engages B cell activation.

$1 \times 10^{6}$ splenocytes were seeded into wells with indicated concentrations of ConA. After $48 \mathrm{~h}$ (dashed) or $72 \mathrm{~h}$ (solid), supernatants were harvested, and IgM analysed by ELISA. Dotted line indicates average background signal from unstimulated cell supernatants. Data are means and SEM from one experiment conducted in duplicate. 
An experiment was conducted to identify the amount of cellular proliferation that has occurred by T cells stimulated with ConA. CFSE is a protein-bound fluorescent dye that is diluted amongst daughter cells during proliferation, reducing the fluorescent intensity of the dye with subsequent generations of cells. Splenocytes were stained with $2.5 \mu \mathrm{M} \mathrm{CFSE}$ and stimulated with $1 \mu \mathrm{g} / \mathrm{mL}$ ConA for 48 or $72 \mathrm{~h}$. At $48 \mathrm{~h}$, very little proliferation had occurred as T cells retained the same CFSE intensity as unstimulated cells. By $72 \mathrm{~h}$, six generations of $\mathrm{T}$ cells were present (Figure 3.12). B cells had not proliferated in this timeframe. Although T cells had not begun proliferation after $48 \mathrm{~h}$ with ConA, cytokines were produced. These data suggest that compounds that alter cellular proliferation may not be toxic to Th cells stimulated with the chosen condition.

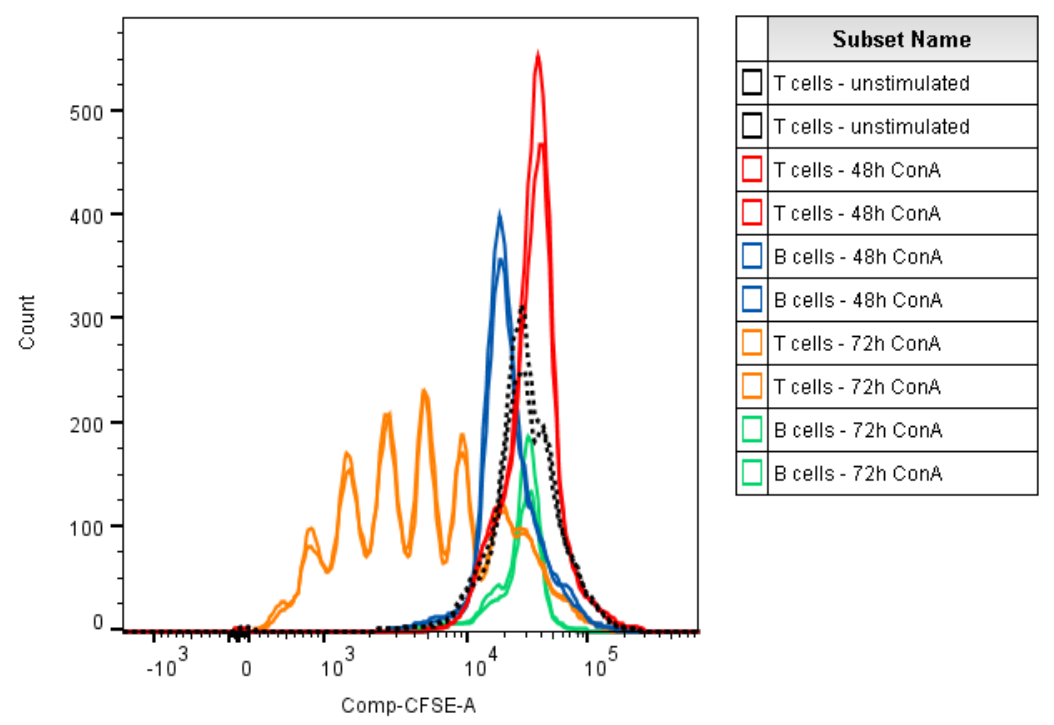

Figure 3.12. $T$ cells proliferate in response to ConA stimulation.

$1 \times 10^{6}$ CFSE labelled splenocytes were seeded into wells of a 96 -well plate and stimulated with $1 \mu \mathrm{g} / \mathrm{mL}$ ConA for indicated times or remained unstimulated. After singlet exclusion, $\mathrm{T}$ cells were identified by staining positively for anti-CD3 antibody, and B220 antibody identified B cells. CFSE intensity was measured from these cell populations (see appendix 9.3.2 for gating strategy). 


\subsubsection{Glatiramer acetate alters Th cytokine production}

Glatiramer acetate is known to alter Th cell cytokine production and was used to test the ability of the assay to detect these changes[68,70,112-115]. Previous research by our lab group used $100-200 \mu \mathrm{g} / \mathrm{mL}$ glatiramer acetate in similar experiments, so a concentration range that included this was used[115]. Alteration of Th cells was seen with $400 \mu \mathrm{g} / \mathrm{mL}$ glatiramer acetate. At this concentration, IL-10 production decreased whilst the other three cytokines all increased (Figure 3.13). These data do not reflect the expected increase in IL-10 from Th cells treated with glatiramer acetate, however, as a change is detected, this assay is a suitable method to determine $\mathrm{T}$ cell alteration by test compounds.

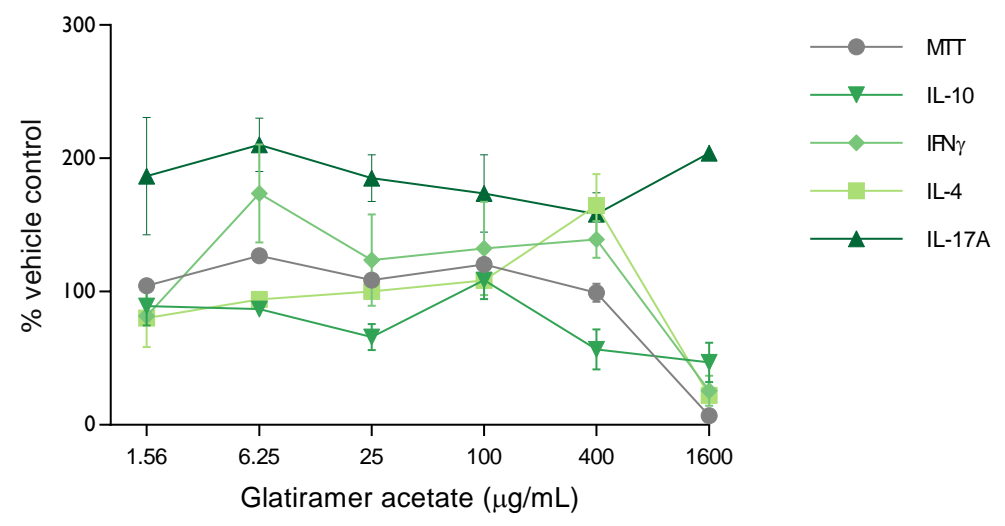

Figure 3.13. $1 \mu \mathrm{g} / \mathrm{mL}$ ConA activation of $\mathrm{T}$ cells is modulated by Glatiramer acetate.

$1 \times 10^{6}$ splenocytes were seeded into wells of a 96-well plate and treated with indicated concentrations Glatiramer acetate or equivalent vehicle control, and stimulated with $1 \mu \mathrm{g} / \mathrm{mL}$ ConA for $48 \mathrm{~h}$. Cell viability was measured by MTT, and cytokines were measured from supernatants by ELISA. Data are means and SEM from one experiment conducted in duplicate.

\subsubsection{Assay Summary}

Splenocyte stimulation with $1 \mu \mathrm{g} / \mathrm{mL}$ ConA for 48 hours elicits T cell activation, including cytokine production, although proliferation does not appear to be underway at this time point. Cytokines representing the four major subtypes of Th are produced with this assay design, and the effects of compounds on $\mathrm{T}$ cell activation can be observed. Within the timeframe of this assay B cell involvement is minimal which suggests changes to assay outputs are solely due to altered Th cell activity. 


\subsubsection{CTL Degranulation}

Cytotoxic T cells (CTL) control intracellular pathogens: infected cells are identified by activated CTLs which degranulate, releasing proteins that induce apoptosis in the infected target cell. These cells also release IFN $\gamma$ upon activation which promotes immune responses against intra-cellular pathogens. CTL also play a role in the immune system's response to cancer where abnormal cells of the self are also destroyed, and CTLs also remove foreign cells.

Controlling these activities can be desirable. Dysfunctional CTLs are responsible for exacerbating autoimmune conditions such as Vitiligo and Type 1 diabetes where CTLs begin recognising structures in the body as threats and begin to destroy them. Suppressing CTL activity has been used in these diseases to alleviate symptoms. CTL suppression is also important for transplant recipients, where CTL recognition and destruction of foreign cells is normal, but not desirable. Enhancing CTL activity may prove desirable for enhancing the immune system's response to tumours; this immunotherapy is under investigation in many laboratories[116]. For the roles CTL play in resolving or causing disease, the ability of compounds to affect CTL activity should be investigated.

Before CTLs can perform their effector roles, they need to be activated by APCs. In the body, DCs carry antigen to secondary lymphoid organs and encounter naïve CTLs that recognise this antigen. This induces CTL activation and these effector CTLs (eCTLs) leave lymphoid organs to seek out infected or abnormal cells. When encountered, CTLs degranulate, releasing cytotoxic granules towards their target; CTLs also produce IFN $\gamma$ upon activation to enhance responses to intracellular pathogens or abnormal cells.

To model CTL activity in vitro, naïve CTLs from mice first need to be activated to generate eCTLs. These eCTLs then need to be treated with compounds and stimulated to assess the effects compounds may have on CTL degranulation and IFN $\gamma$ production. 


\subsubsection{Optimisation}

Studies of degranulation have been extensively reviewed by Betts and Koup[117]. Previously, to study CTL degranulation a culture of APCs would be 'pulsed' with cognate peptide (i.e. one that CTL recognise as pathogenic/abnormal) to load MHC Class I. These APCs would be co-cultured with naïve CTL to generate eCTL. After sufficient activation, these CTLs will be ready to kill target cells. A separate cell culture would be labelled with CFSE or ${ }^{51} \mathrm{Cr}$ (chromium) and loaded with peptide to become target cells (i.e. cells to be destroyed). Effector CTLs and target cells would be cultured together eliciting degranulation in the CTL. Degranulatory activity would be measured indirectly by CFSE loss in target cells populations, or by measuring the chromium released from killed cells[118-120].

After it was observed that surface CD107a expression is associated with degranulation, target cells no longer needed to be labelled[118]. CD107a is found on the inner membrane of cytolytic granules in CTL. During degranulation, this lysosome membrane fuses with the cell membrane and CD107a becomes accessible for extracellular labelling on the active CTL[117]. This allows for direct measurements of CTL degranulation, and with this activity marker there is no need to label target cells to measure degranulation. Only one separate culture of cells is required: peptide-loaded APCs would be co-cultured with naïve CTLs to generate eCTL, and this co-culture would continue until eCTLs killed these APCs. This design was adapted for use in this project.

OT-1 mice are transgenic mice where most, if not all CTLs will recognise and respond to cells that display the SIINFEKL peptide, a component of the OVA protein, on MHC Class I[121,122]. OT-I CTLs remain in a naïve state in these mice as the OVA protein is not normally encountered. These mice were available for use and were investigated to establish a protocol for an in vitro assessment of CTL degranulation. In this section, to minimise confusion, the activation of naïve CTLs is called "priming" and the initiation of degranulation is called "stimulation".

It was hypothesised that OT-1 splenocyte preparations could provide both the naïve CTLs, and a sufficient number of B cells that would act both as APCs and target cells. This takes advantage of SIINFEKL's ability to load externally into MHC Class I, not requiring cross- 
presentation by a dendritic cell[123]. If successful, this would remove the need for culturing APCs, reducing the resources required for assessing CTL activity.

To assess eCTL generation, splenocytes from OT-1 mice were cultured with SIINFEKL peptide, with and without IL-2, as suggested in the literature[124-126]. Peptide-loaded B cells would engage naïve CTL, and IL-2 would promote CTL proliferation. Unstimulated cells were included as a negative control, as well as an IL-2 only control to assess if this could be used alone to boost numbers through cell proliferation prior to priming[127]. The in vitro activation of naïve CTLs was expected to take between 48 - 72 hours based on previous methodologies[122,128,129]. With this information, an experiment was designed to investigate the priming conditions required to generate eCTLs in vitro. By observing the size increase when T cells are activated, active CTLs cells appeared by 24 hours of culture, and persisted through to the next day when SIINFEKL was included, though cell morphology in these cultures had changed by day three (Figure 3.14, A)[130,131]. Samples of each priming condition were stained with the lymph node-homing marker CD62L, and egression/effector marker CD44, to assess eCTL generation by flow cytometry; cells were also stained for CD3 and CD8 to identify CTL. Proportions of CD62L ${ }^{+}$CD44- naïve; $\mathrm{CD}_{2} \mathrm{~L}^{+} \mathrm{CD} 44^{+}$central memory; and CD62L-CD44 ${ }^{+}$effector CTL were assessed[132] (see appendix 9.3.3 for gating strategy). By $48 \mathrm{~h}$ the CTL population shifted to the effector phenotype in the presence of SIINFEKL; IL-2 had minor influence on effector generation at this time point (Figure 3.14, B). Though the proportion of effector CTLs at $72 \mathrm{~h}$ looks promising, the viability of all culture conditions is very low (Figure 3.14, C). Based on cell count data, activating CTLs with SIINFEKL and IL-2 resulted in the highest yield of eCTL at $48 \mathrm{~h}$ (Figure 3.14, D). However, for simplicity, SIINFEKL alone for $48 \mathrm{~h}$ generated sufficient quantities of eCTL without the need for the cytokine reagent.

During flow cytometry analyses of this experiment, it was apparent that staining for surface markers CD3 and CD8 was unnecessary. After identifying the lymphocyte population by forward- and side-scatter properties, the $\mathrm{CD}^{+} \mathrm{CD}^{+}$component averaged $98.3 \%$ (SD 0.29\%) across 50 samples, so staining with these marker was deemed unnecessary: it only added reagent costs and time to the assay (see figure in appendix 9.3.4). 

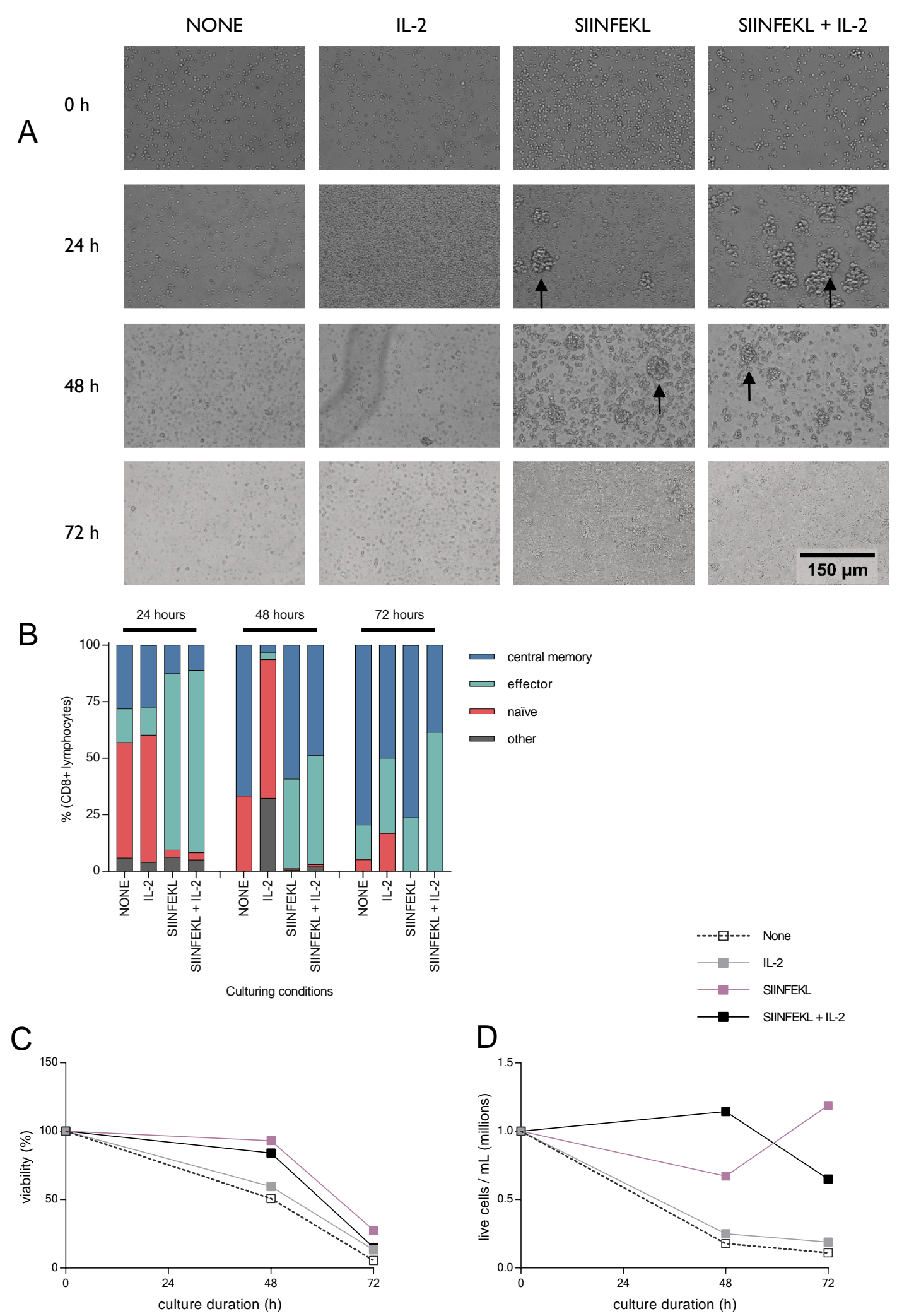

Figure 3.14. SIINFEKL alone adequately generates eCTL after $48 \mathrm{~h}$.

OT-I splenocytes were cultured at $1 \times 10^{5}$ cells/well in a 24 well plate alone, or with $100 \mathrm{U} / \mathrm{mL}$ IL-2, 10nM SIINFEKL, or both. Large, activated CTL can be identified by light microscopy (arrows identify examples; A). Flow cytometry was used to determine naive, central memory, and effector CTL populations as percentages of the lymphocyte gate; data were collected from $100 \mu \mathrm{L}$ of the four culturing conditions (B). Cell viability (C) and cell counts (D) were assessed by trypan blue. Data are from one experiment and presented as averages; cultures were conducted in duplicate, with triplicate measures from each culture. 
In this same experiment, cells were also stained for CD107a to determine if cells were degranulating during the priming process. This was found to be the case. The inclusion of a rest period after eCTL generation was investigated to see if CD107a expression, i.e. degranulatory activity, would decline (Figure 3.15). After an 18-hour incubation in fresh media without SIINFEKL, cells appeared to enter a resting state, identified by low levels of the T cell activation marker, CD25, and CD107a. It was important to reset degranulatory activity before stimulation to increase the difference between 'baseline' and stimulated expression of CD107a to improve assay sensitivity, i.e. the ability to detect changes in degranulation. This rest period is assumed to see depletion of SIINFEKL from the system.
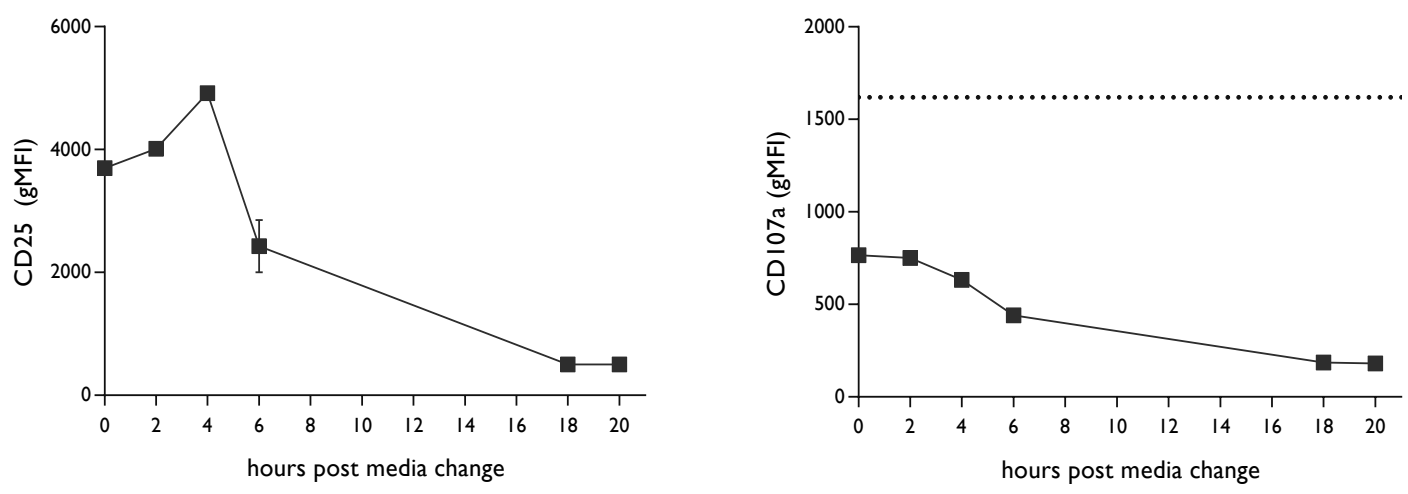

Figure 3.15. An overnight rest resets eCTL to a resting state after priming.

To bring primed eCTL to a resting state - so that degranulation could be assessed with greater resolution - it was determined that cells need $18 \mathrm{~h}$ (overnight) in fresh media to rest and reset. After resuspending primed eCTL in fresh media (without SIINFEKL), CD25 expression (left; T cell activation), and CD107a surface expression (right) declines with time. Dashed line indicates marker expression after $2 \mathrm{~h}$ stimulation to initiate degranulation. Data are means and SEM from one experiment conducted in duplicate

An experiment was performed to identify optimal concentrations of SIINFEKL to use for generating eCTLs (priming) as the concentrations used thus far have come from the literature. In this experiment, 100,000 splenocytes were seeded into wells of a 96-well plate and primed with 1, 10, 50 or $100 \mathrm{nM}$ SIINFEKL for 48 hours. Of the priming concentrations tested, $100 \mathrm{nM}$ SIINFEKL reduced cell viability. Lower amounts of SIINFEKL resulted in a greater proportion of generated eCTL; eCTLs were identified by their larger cell size (Table 3.1). 
Cells from this experiment were then stimulated with different concentrations of SIINFEKL for two hours to induce degranulation; this is indicated in the literature to be a suitable stimulation period[118]. It was assumed that B cells persisted through CTL priming and could be reloaded with SIINFEKL to engage CTLs. Degranulation was successfully initiated, and the extent of degranulation was measured by flow cytometry. Stimulating-peptide concentration had little impact on degranulation, however the priming concentration appeared to affect the degranulatory activity of eCTL as cells primed with higher concentrations of SIINFEKL had enhanced degranulatory ability (Figure 3.16). Whilst it appears that priming cells with 50 - 100 nM SIINFEKL would be ideal - the cells degranulate well - the yield would be relatively low (Table 3.1). Based on high culture viability, eCTL yield and degranulatory activity, $10 \mathrm{nM}$ SIINFEKL remained as the priming concentration. For stimulation, 25 nM SIINFEKL was selected for no other reason than to add it in excess.

Table 3.1. eCTL generation with different priming and stimulation peptide concentrations

\begin{tabular}{|c|c|c|c|c|}
\hline $\begin{array}{c}\text { Priming SIINFEKL } \\
\text { conc. }(\mathrm{nM})\end{array}$ & $\begin{array}{c}\text { Viability } \% \\
\text { (all cells) }\end{array}$ & $\begin{array}{c}\text { Number of live } \\
\text { cells }\left(\times 10^{5}\right)\end{array}$ & $\begin{array}{c}\text { Number of live } \\
\text { eCTL }\left(\times 10^{5}\right)\end{array}$ & \% eCTL \\
\hline 1 & 84.4 & 2.5 & 2.2 & 88 \\
\hline 10 & 82 & 1.4 & 1.3 & 93 \\
\hline 50 & 80 & 1.1 & 0.8 & 76 \\
\hline
\end{tabular}

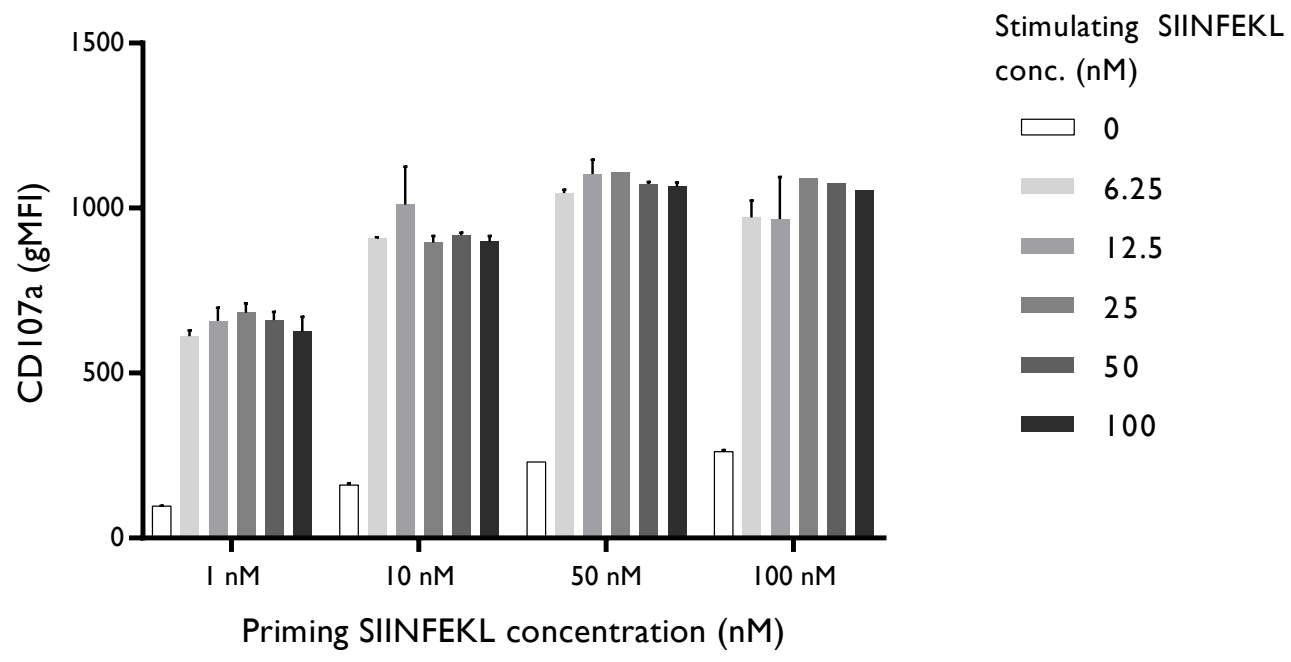

Figure 3.16. Priming SIINFEKL concentration may affect CTL functionality.

100,000 CTLs were seeded into wells of a 96-well plate. The indicated concentrations of SIINFEKL for priming (48 h) and stimulation were used (2 h), and degranulatory function assessed by measuring CD107a expression by flow cytometry (see Figure 3.22 for gating). Data are means and SEM from one experiment conducted in duplicate. 
Lastly, to fine-tune the assay design, stimulation duration was investigated. Primed OT-1 splenocyte cultures enriched for eCTL were stimulated with 25 nM SIINFEKL to initiate degranulation. CTLs were assessed for surface CD107a expression after 1, 2 and 4 hours (Figure 3.17). Degranulation had occurred by the earliest time point assessed and similar levels of CD107a were observed at two and four hours post stimulation. IFN $\gamma$ from stimulated eCTL supernatants was detectable after two- and four-hour stimulation (Figure 3.18). To collect more information on CTL activity, i.e. degranulation and IFN $\gamma$ production, the two-hour stimulation period was selected. To summarise the assay design, eCTLs are generated from OT-1 splenocytes incubated with $10 \mathrm{nM}$ SIINFEKL for $48 \mathrm{~h}$. Cells are then rested overnight. To initiate degranulation eCTL cultures are stimulated with $25 \mathrm{nM}$ SIINFEKL for two hours. Throughout this process, B cells in cultures act as APCs and target cells.

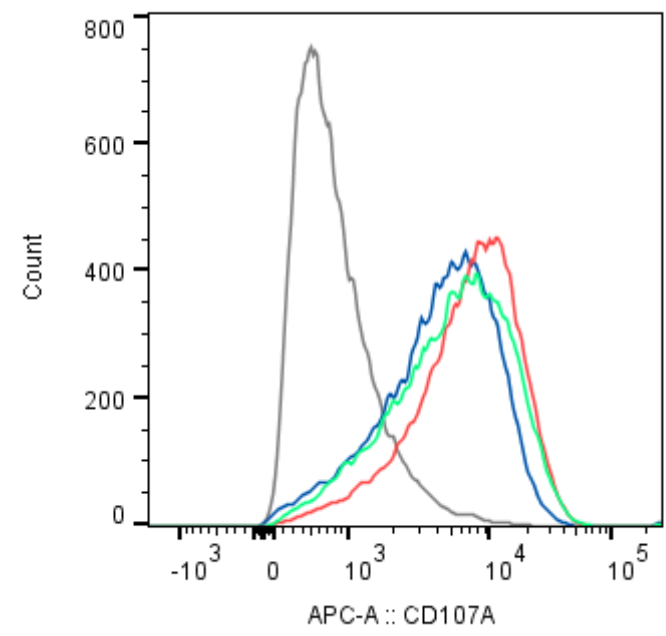

\begin{tabular}{|c|c|c|c|}
\hline & TUBE NAME & Subset Name & Count \\
\hline & 4 h stimulation & Q2: CD3E+, CD8A+ & 28774 \\
\hline & 2 h stimulation & Q2: CD3E+, CD8A+ & 27090 \\
\hline & $1 \mathrm{~h}$ stimulation & Q2: CD3E+, CD8A+ & 28554 \\
\hline & unstimulated & Q2: CD3E+, CD8A+ & 29539 \\
\hline
\end{tabular}

Figure 3.17. Degranulation occurs as early as $1 \mathrm{~h}$ post stimulation.

$1.4 \times 10^{5}$ eCTL were seeded into wells of a 96 well plate and stimulated to degranulate by adding $25 \mathrm{nM}$ SIINFEKL to cultures. CD107a was measured on eCTL by flow cytometry after 1 (blue), 2 (red) or 4 (green) hours. CD107a expression was also measured on unstimulated cells (grey). The intensity of CD107a on stimulated cells did not differ significantly between the different time points but was much higher than unstimulated cell CD107a expression. 


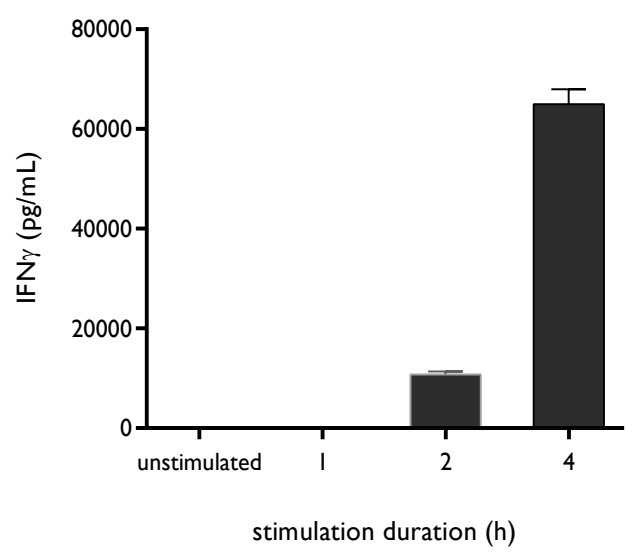

Figure 3.18. IFN $\gamma$ produced during eCTL activation is measurable after 2 h stimulation.

$1.4 \times 10^{5}$ eCTL were seeded into wells of a 96 well plate and stimulated to degranulate by adding $25 \mathrm{nM}$ SIINFEKL to cultures. After 1,2, or 4 hours stimulation, supernatants were collected and analysed for IFN $\gamma$. Data are means and SEM from one experiment conducted in duplicate.

\subsubsection{Colchicine inhibits CTL degranulation and IFN $\gamma$ production}

Colchicine is a microtubule destabiliser which has previously been used to impair degranulation in CTL. It does this by disrupting the mechanisms through which organelles rearrange, and export vesicles out of the cell[133]. Effector CTLs were treated with various concentrations of colchicine for four hours, before stimulating with $25 \mathrm{nM}$ SIINFEKL for two hours (Figure 3.19). Cells were then analysed for CD107a expression by flow cytometry. CTL degranulation activity dropped to $50 \%$ of vehicle-treated CTL with $1 \mu \mathrm{M}$ colchicine, and IFN $\gamma$ was similarly inhibited at this concentration. This experiment indicates the assay's suitability to demonstrate CTL activity, and its ability to detect the inhibition of this activity by compound. 


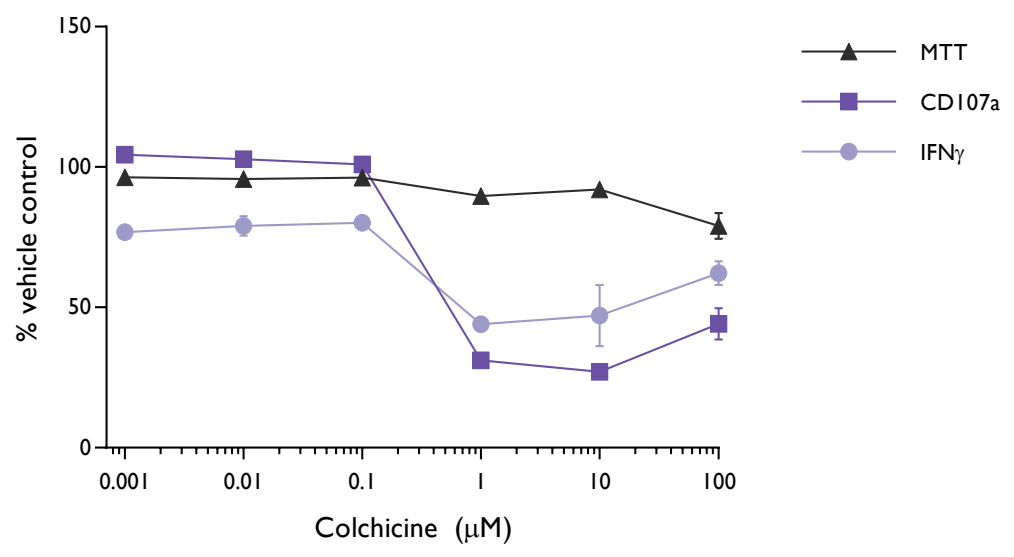

Figure 3.19. Microtubule depolymerisation due to Colchicine inhibits degranulation.

$1.4 \times 10^{5}$ eCTL were seeded into wells and pre-treated with Colchicine or vehicle control for 4 h. $25 \mathrm{nM}$ SIINFEKL was added to wells to initiate degranulation alongside further treatments to maintain concentration for a further $2 \mathrm{~h}$. $1 \mu \mathrm{g} / \mathrm{mLCD} 107 \mathrm{a}$ :APC was added to wells at this time to label degranulating cells. Supernatants were analysed by ELISA and cells subjected to flow cytometry to determine degranulation. Colchicine-treated eCTLs were subjected to MTT for viability assessment after 4 hours in a parallel experiment. Data are means and SEM from one experiment conducted in duplicate.

A 20-hour pre-treatment period was also investigated as this overnight treatment may prove more convenient, practically, when running multiple assays or multiple plates. In this experiment, colchicine's ability to inhibit degranulation is not observed, and only appears to decline alongside diminishing cell viability (Figure 3.20). Although data were collected, very few cells were detected during flow cytometry analyses and, after observing cells via microscope, it appeared that cells were unhealthy. It was hypothesised that the sustained treatment duration was too long for CTLs to survive without supporting signals from other cells, including IL-2[134,135]. IL-2 levels dropped between 4 and 20 hours after cells were plated; IL-2 levels at $20 \mathrm{~h}$ were 15\% the levels measured at $4 \mathrm{~h}$ (Figure 3.21). Due to the difficulty in maintaining live eCTLs during the extended treatment, and because the shorter treatment duration can detect the inhibition of degranulation by a known inhibitor, further optimisation of the 20-hour treatment duration was not undertaken. 


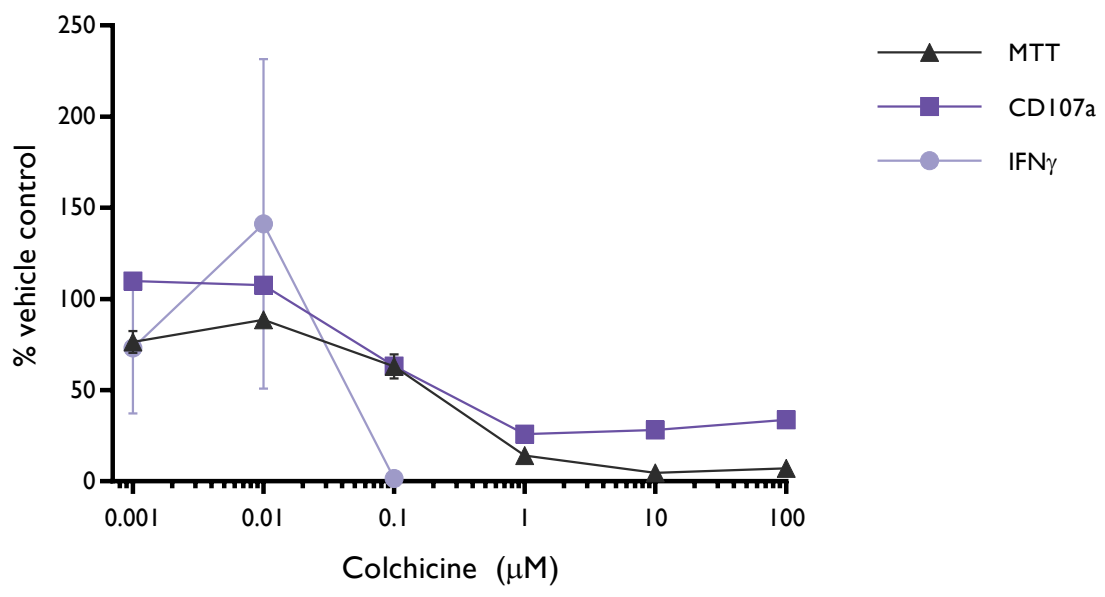

Figure 3.20. The degranulation assay is not informative with a 20-hour drug pre-treatment.

$1.4 \times 10^{5}$ eCTL were seeded into wells and pre-treated with Colchicine or vehicle as a control for $20 \mathrm{~h} .1$ $\mu \mathrm{g} / \mathrm{mL}$ CD107a:APC was added to wells with $25 \mathrm{nM}$ SIINFEKL and further treatment to maintain concentration for a further $4 \mathrm{~h}$. Supernatants were analysed by ELISA and cells subjected to flow cytometry to determine degranulation. Data are means and SEM from one experiment conducted in duplicate.

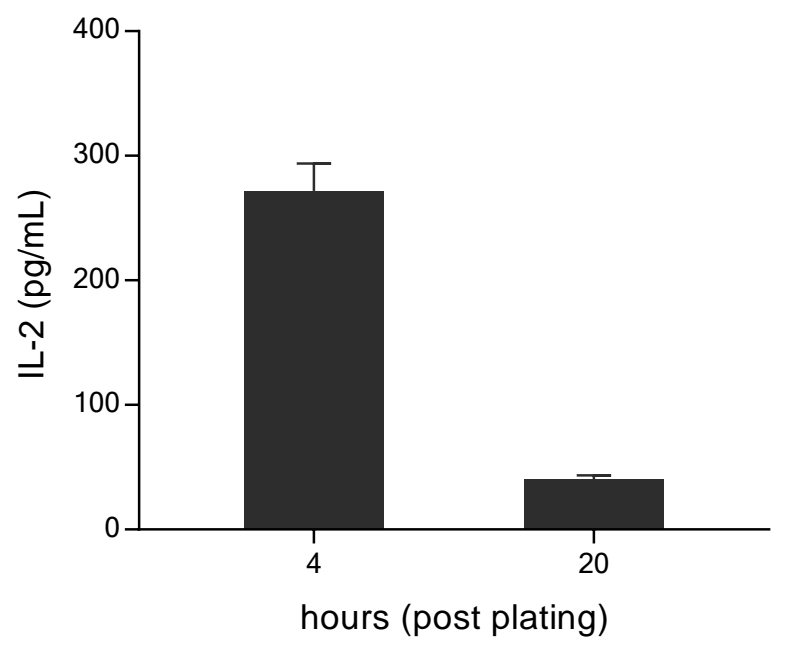

Figure 3.21. IL-2 levels in CTL cultures dropped between 4 and $20 \mathrm{~h}$ after plating.

$1.4 \times 10^{5}$ eCTL were seeded into wells and pre-treated with vehicle (EtOH) for four or 20 hours. Harvested supernatants were analysed for IL-2 by ELISA. Four-hour and 20-hour data are each from separate experiments, and data are means and SEM of six replicates. 


\subsubsection{Summary}

A 48-h incubation with $10 \mathrm{nM}$ SIINFEKL peptide alone effectively generated eCTL from an OT-1 splenocyte preparation. After resuspending cultures in fresh media without SIINFEKL overnight, cultures are plated and pre-treated for four hours with test compound. Degranulation is initiated with the addition of $25 \mathrm{nM}$ SIINFEKL for two hours, and CD107a is added during stimulation to measure degranulation by flow cytometry. A gating strategy for this experiment is included below (Figure 3.22).

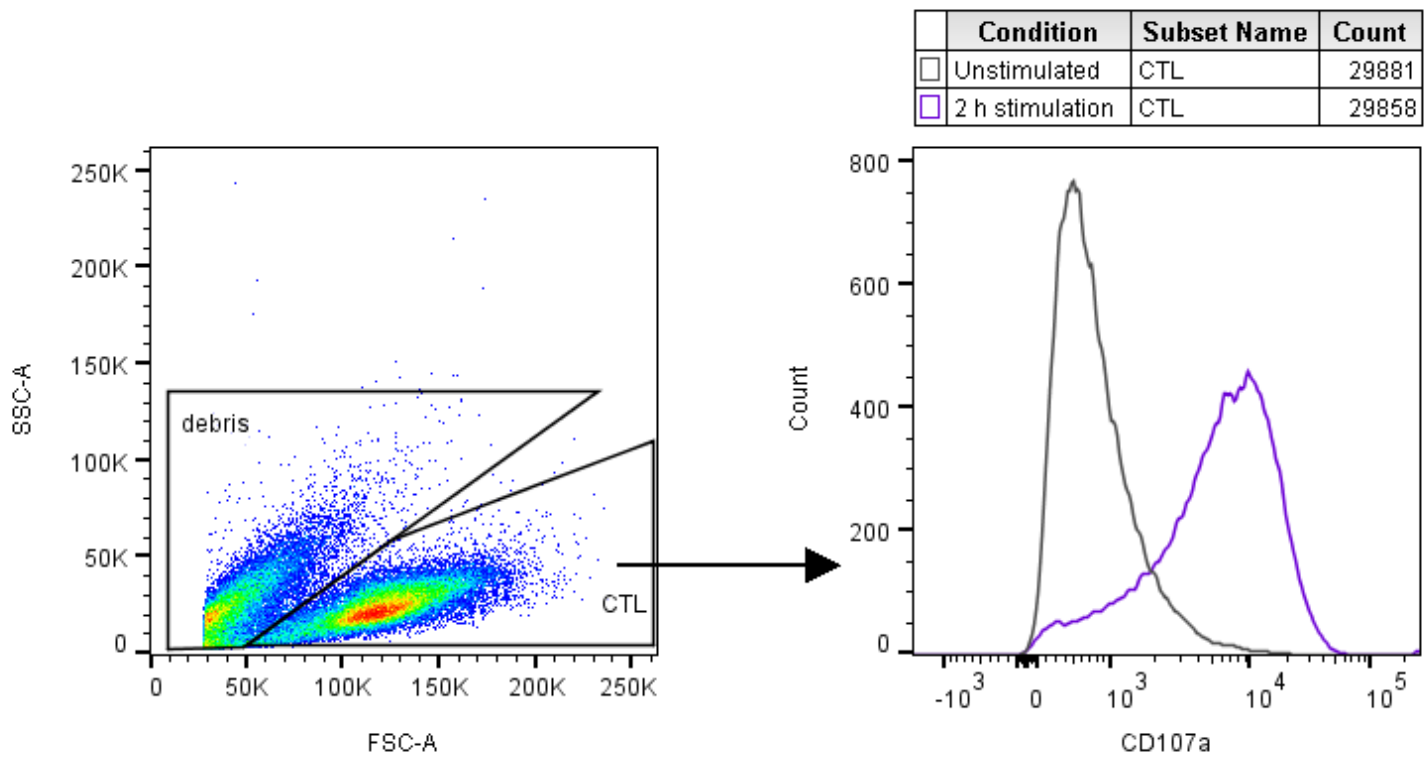

Figure 3.22. Gating strategy to measure CD107a, or degranulation, by CTL.

After doublet exclusion (not shown), CTLs were identified from debris by FCS and SSC. CD107a measures degranulation, demonstrated by the increased CD107a signal when cells are stimulated (purple) compared to unstimulated (grey) cells. 


\subsubsection{B cell activity using LPS}

B cells are known for their production of antibodies upon activation, and these antibodies have important roles in the immune system. These antibodies bind to pathogens and any toxins they produce during infection leading to their neutralisation and destruction. B cells have also been implicated in immune disorders, notably for the production of autoreactive antibodies which erroneously bind to proteins of the body. This contributes to Systemic Lupus Erythematosus, where B cells produce autoantibodies that bind nuclear proteins. This causes cell injury to the point tissues are damaged.

Common treatments to alter dysfunctional B cells do so through suppression; ibrutinib and rituximab both deplete B cells. There is room for novel treatments that alter B cell activities as an alternative to outright B cell depletion. B cells have other important activities, including antigen presentation and cytokine production, which may not be defective or dysfunctional during autoantibody production, and leaving these normal activities intact help maintain the integrity of the immune system[60,136,137]. Finding compounds that can specifically target individual B cell functions may be beneficial in treating certain autoimmune conditions.

Pokeweed mitogen, Anti-B cell receptor (anti-IgM) antibodies and anti-CD40 (or CD40L) have been used to stimulate B cells in vitro[138]. Upon activation, B cells produce antibody and cytokines. Before investigating these options, LPS-stimulation of B cells was investigated. LPS was readily available as it is used for macrophage activation, and has been used previously to stimulate B cells[44,59]. For these reasons, B cell activation with LPS was investigated. As this method ended up being suitable for modelling B cell activation, other stimulation methods were not investigated.

\subsubsection{Optimisation}

Activated B cells can produce a wide range of cytokines and these cytokines delineate the subtypes of B cells which occupy different niches in the immune system. Some have a regulatory role by producing IL-10, whilst others can enhance inflammation by producing TNF $\alpha$ and IL-12[60,139]. B cell production of IL-10 and IL-6 has previously been observed with LPS stimulation so these cytokines were measured alongside IgM during assay optimisation[136,140]. The IgM isotype was selected as it is the first antibody isotype produced upon activation. 
Splenocytes were stimulated with $0.003-10 \mu \mathrm{g} / \mathrm{mL}$ LPS for 48 and $72 \mathrm{~h}$ to investigate which level of LPS elicits half-maximum B cell activities, and confirm which cytokines are produced with this type of activation (Figure 3.23). Peak IgM production was reached with $1 \mu \mathrm{g} / \mathrm{mL}$ LPS, with half-maximum production elicited by $0.1 \mu \mathrm{g} / \mathrm{mL}$ LPS for both time points (Figure 3.23, A). Half-maximum IL-10 production was also elicited by $0.1 \mu \mathrm{g} / \mathrm{mL}$ LPS at $48 \mathrm{~h}$, though this may not be true for the longer duration as peak production was not observed (Figure 3.23, B). Peak production of IL-6 is not observed in this assay at either time-point, though stimulation with $0.1 \mu \mathrm{g} / \mathrm{mL}$ LPS induces IL-6 production at a level where compound-induced changes to this cytokine should be detectable (Figure 3.23, C). The 48-hour assay duration was selected for B cell stimulation with $0.1 \mu \mathrm{g} / \mathrm{mL}$ LPS as this sees the mid-level production of IgM and cytokines, and the protocol only differs from the helper T cell assay by stimuli.
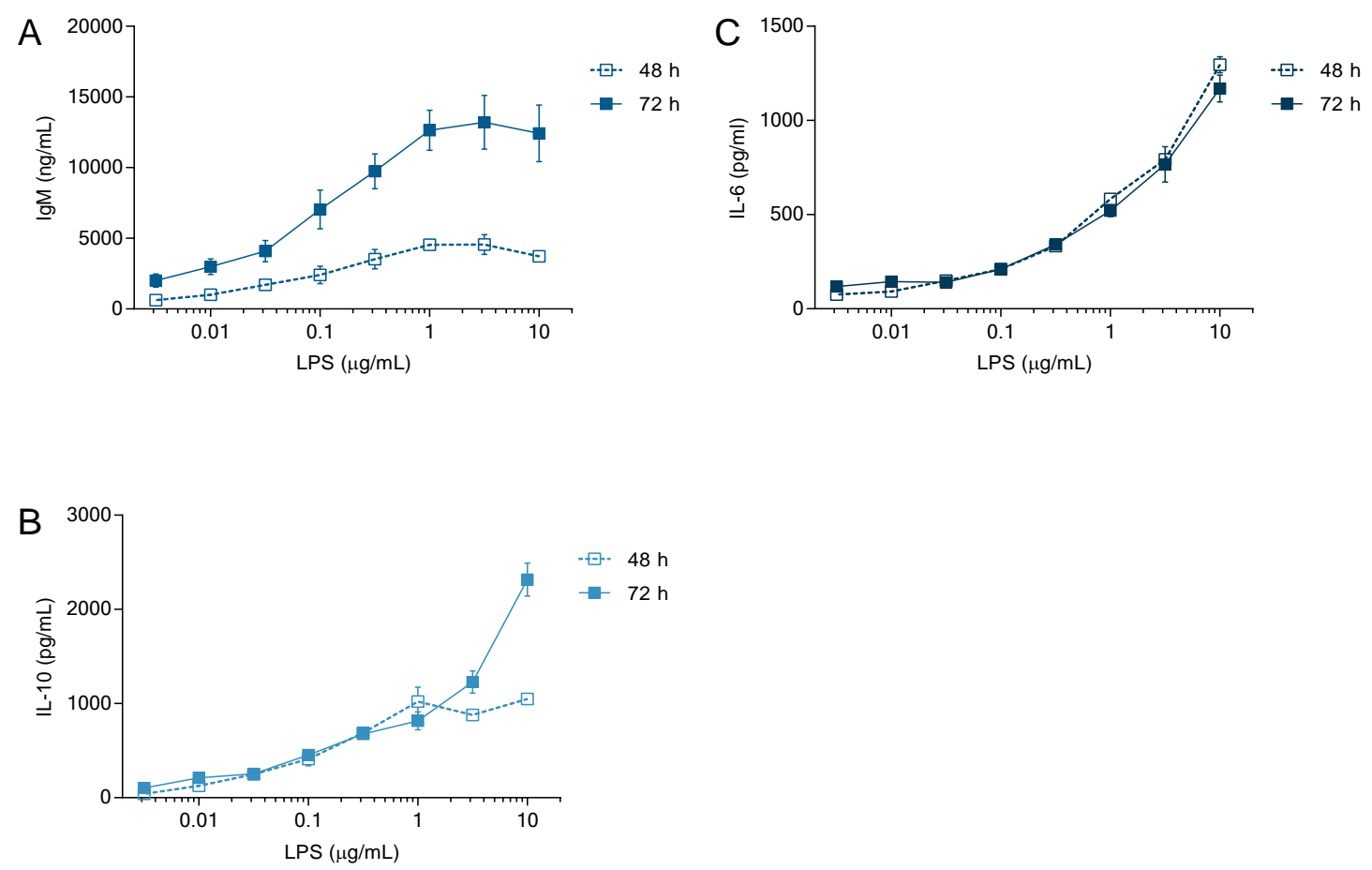

Figure 3.23. LPS activates B cells in splenocytes to produce IgM, IL-10 and IL-6.

$1 \times 10^{6}$ splenocytes were seeded into wells of a 96-well plate and stimulated with indicated concentrations of LPS. After 48 h (dashed) or 72 h (solid), supernatants were harvested and IgM (A), IL-10 (B) and IL-6 (C) were measured by ELISA. Data are means and SEM from one experiment conducted in duplicate. 
It is commonly known that B cells proliferate upon activation before differentiating into antibody-producing plasma cells, and an experiment was run to assess this. CFSE-labelled splenocytes were stimulated with $0.1 \mu \mathrm{g} / \mathrm{mL}$ LPS for $48 \mathrm{~h}$. B cells did not undergo proliferation within the 48-h time period: activated B cells retained the same dye intensity as unstimulated cells (Figure 3.24). $\quad \mathrm{T}$ cell proliferation was also assessed in this experiment for two reasons. First, the T cell population of ConA-stimulated splenocytes serves as a positive control to identify proliferation using CFSE dye (indicated in grey); secondly, to assess if LPS stimulation of B cells causes downstream T cell activation within $48 \mathrm{~h}$. If this was true, the measured IL-10 may be coming from active T cells. However, $\mathrm{T}$ cells during LPS stimulation did not proliferate, suggesting that this stimulation is specific for B cells.

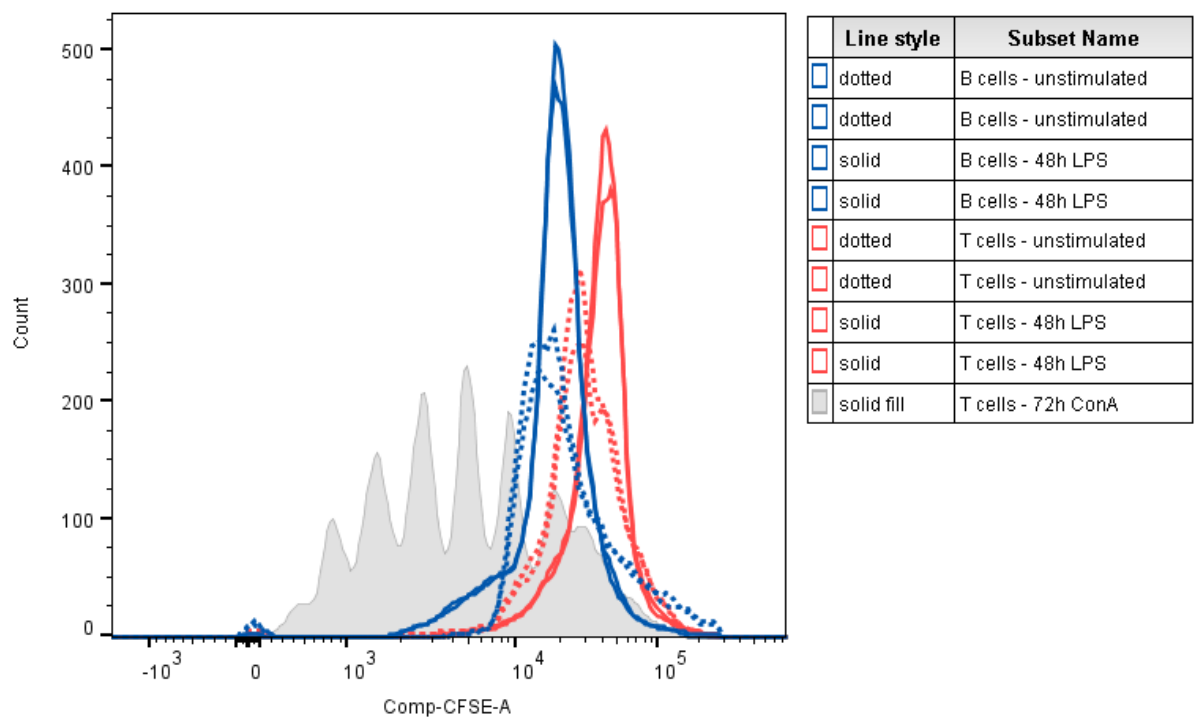

Figure 3.24. B cells do not proliferate with LPS stimulation.

CFSE-stained splenocytes were seeded at $1 \times 10^{6}$ cells per well and stimulated for $48 \mathrm{~h}$ with $0.1 \mu \mathrm{g} / \mathrm{mL} \mathrm{LPS}$ (solid lines) $1 \mathrm{ug} / \mathrm{mL}$ ConA (grey fill) or remained unstimulated (dotted). B cells (blue) were identified by positive staining for B220 and T cells (red) were identified by positively staining for CD3. Fluorescence intensity of CFSE of these populations were assessed by flow cytometry. Data are from one experiment conducted in duplicate. (see appendix 9.3.2 for gating strategy) 


\subsubsection{Inhibiting LPS-stimulated B cell activity with cycloheximide}

Cycloheximide, a protein synthesis inhibitor, was used to ensure an expected decrease in antibody and cytokine production could be detected with this assay. Antibodies and cytokines are small proteins that are synthesised upon activation, and cycloheximide should inhibit this process. Again, a wide range of concentrations were used to ensure this inhibitory activity was observed. The activated B cells were susceptible to the drug, with viability decreasing significantly (75\% control or lower) at concentrations above $0.01 \mu \mathrm{M}$ cycloheximide. Where viability is largely unaffected $(0.001-0.01 \mu \mathrm{M})$, IgM and IL-10 production was reduced by half; IL-6 remained relatively unaffected by treatment (Figure 3.25). This demonstrated the assay’s ability to detect changes to B cell activities.

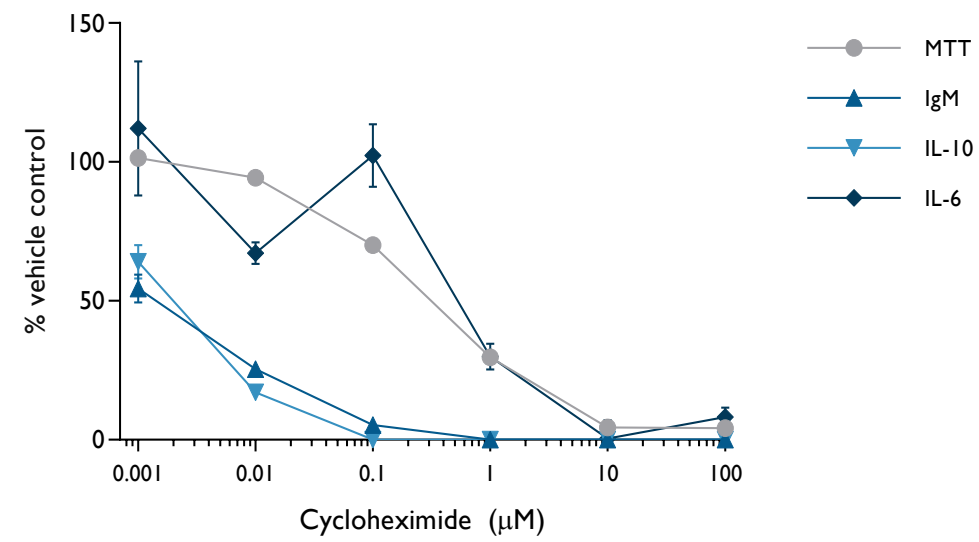

Figure 3.25. The effects of the protein synthesis inhibitor on B cells is detectable in this assay. $1 \times 10^{6}$ splenocytes were seeded into wells of a 96 -well plate and stimulated with $0.1 \mu \mathrm{g} / \mathrm{mL}$ LPS for $48 \mathrm{~h}$ with the addition of indicated concentrations of cycloheximide, or vehicle as a control. Cells were subjected to MTT for viability, and supernatants analysed by ELISA for IgM, IL-10 and IL-6. Cells remain viable at $0.001-0.01 \mu \mathrm{M}$ cycloheximide, where levels of IgM and IL-10 were decreased. IL-6 release by stimulated B cells was closely related to the viability of cells. Data shown are means and SEM from one experiment conducted in duplicate.

\subsubsection{Summary}

$0.1 \mu \mathrm{g} / \mathrm{mL}$ LPS stimulation of splenocytes for $48 \mathrm{~h}$ activates B cells. This results in IgM, IL-10 and IL-6 production. These outputs are measurably altered by cycloheximide, which serves as a good positive control. 


\subsection{Discussion}

Having the right assays to identify drug activity is the key component when building a screening platform or strategy. This chapter saw the optimisation and/or design of assays that detect changes to immune cell activity in a medium-throughput manner. With these assays, compounds can be tested en masse for activity on macrophages, T cells and B cell functions including proliferation, the production of cytokines, antibody and NO, as well as phagocytosis, cell motility and degranulation.

After confirming appropriate priming and stimulation conditions for the macrophage cell line RAW264.7, a change in activity was detected when these cells were activated in the presence of Risperidone. However, the observed changes did not resemble what is reported in the literature. Repeating the experiment with BMDM saw Risperidone-induced changes that concurred with published findings. The observed reduction of IL-12 and increased IL-10 production by activated macrophages treated with risperidone matched previous studies that used the same assay design as used in this experiment. Although it would appear more appropriate to replace RAW 264.7 cells with BMDM, the latter cell type is far more resource-intensive to source and culture than the cell line. Though this assay design is adequately sensitive enough to detect an alteration to macrophage activation, further testing should determine to what depth the data from RAW 264.7 cells can be used to assess macrophage activity changes, i.e. to assess whether the unexpected alteration of IL-12 production being the only unexpected activity of these RAW 264.7 cells.

Using pHrodo bioparticles provides an easy method to investigate phagocytic activity, and high-throughput flow cytometry suits compound screening. This assay was optimised to reduce reagent use, and increasing the incubation time offered the ability to measure changes to concurrent cytokine production. The assay design serves its intended purpose, and LatA inhibited phagocytosis as previously described in the literature. It is worth noting that this assay uses a pH-sensitive dye to indicate that phagocytosis has taken place. A decreased fluorescent signal could also be caused by the loss of lysosome acidification: if the $\mathrm{pH}$ of the lysosome cannot decrease, the fluorescent signal from the pHrodo particle cannot be produced. Therefore, any alteration of phagocytic activity that is detected by this method could be altered cell ability to engulf particles, or altered phagolysosomal fusion and acidification. 
The ORIS wound filling assay offered a medium-throughput, simple to use format with which the alteration of cell motility by test compounds can be investigated. A preliminary trial run to assess motility using the scratch assay identified that macrophages move slowly, and that proliferation by RAW 264.7 cells interfere with motility assessment. After substituting RAW 264.7 cells for BMDM to remove this interference, and moving to the ORIS format, changes to cell motility were observed with LatA. Though the plate is a medium-throughput format, the image acquisition was a slow process because it involved use of a light microscope. High-throughput imaging systems exist and should be explored to accelerate this process. This assay does not measure chemotaxis, an important factor for immune cell motility. Whilst cell motility is a component of chemotaxis, the latter offers another mechanism through which immune cell motility can be altered by compounds. Inhibiting chemotaxis is the mechanism through which Fingolimod delivers therapeutic benefit to sufferers of multiple sclerosis[141]. The ORIS assay was selected for its ease of use, but with the limitation that compounds that alter an immune cell's ability to recognise and respond to chemotactic signals are not assessed.

The helper $\mathrm{T}$ cell assay design adequately models $\mathrm{T}$ cell activation and differentiation. Based on cytokine production, all Th subsets were generated by ConA stimulation, and subset bias was altered in the presence of Glatiramer acetate. This drug may not be the best control to induce cytokine changes for this assay and subsequent experiments may identify a better Th cell modulator. Despite this, the assay is a suitable method by which compoundinduced changes to Th cytokine production, and therefore subset bias, can be assessed. Because this assay uses a splenocyte culture, both $\mathrm{B}$ and $\mathrm{T}$ cells are present during stimulation. Of the two stimulation durations tested during optimisation, the shorter $48 \mathrm{~h}$ stimulation sees the activation of $\mathrm{T}$ cells, whilst hallmarks of B cell activation were not detected. This suggests this assay detects cytokine production from T cells only.

Similar to the helper T cell activation assay, B cell activation was achieved through LPS stimulation. Splenocytes stimulated with LPS activate regulatory B cells which produce IgM, IL-10 and IL-6. This assay does not investigate a compound's ability to alter B cell phenotypes, which would require the assessment of a wide range of other cytokines. Nor does the assay measure other antibody isotypes which is important to note as class switching can occur on the second day after LPS stimulation[142]. These aspects of B cell 
activity are interesting and are worth investigating in follow-up experiments if changes to B cells are observed.

As potent killers of infected and abnormal cells, CTL activity was also represented in this set of assays. Protocols for culturing CTLs were explored, and a single-culture methodology was developed in this project; this reduced the complexity and costs of performing degranulation assays. As the inhibition of degranulation was observed with colchicine as expected, this assay adequately identifies CTL modulation.

Though many of these assays were optimised to be cost-effective, for both the phagocytosis and degranulation assays there is a need to treat two sets of cells in parallel. One set would be subjected to flow cytometry analyses, whilst viability would be analysed by MTT assay using the second plate. The use of fluorescent viability dyes may be able to eliminate the need for the second plate by assessing viability on the same cells that phagocytosis or degranulation is assessed with. If successful, this would reduce the resources required to perform both these assays, and thus warrants further investigation.

\subsection{Summary}

To summarise, together these assays measure the activities of immune cells which play important roles in the immune response and are also implicated in the progression of autoimmune and inflammatory diseases. The assay designs are appropriately sensitive to detect changes induced by compounds: all assay outputs were altered by relevant positive controls with the exception of NO production by activated macrophages. The assays were designed to be cost effective and were simplified where possible. Together, they provide the foundation from which a screening strategy to detect immunomodulation can be built. This is the focus of the following chapter 


\section{Chapter 4: \\ Building the screening strategy}




\subsection{Introduction}

In the previous chapter, individual assays were optimised to detect changes to various immune cell treated compounds. The next step was to arrange these assays into an efficient and logical screening strategy that assesses the immunomodulatory activity of compounds under investigation, or "test compounds". To build the screening strategy, it was important to understand how each individual assay relates to the others. For example, one assay output may reliably predict a change to another immune function, so not every output would need to be assessed. If so, it is essential to determine how uniquely informative each of the selected assays, and assay outputs, are to improve screening efficiency.

In this chapter, the optimised assays from Chapter 3 were used to test a set of known bioactive compounds. Subsequently, the data from screening these compounds was used to reverse-engineer an optimal compound screening strategy. A hierarchy within the assays was hypothesised to exist: certain assays would be more informative than others, and the type of changes observed in these more sensitive assays would provide robust information that can be used to direct further in-depth investigations through the remaining assays, and beyond. That is, the first layer of assays would be able to reproducibly and sensitively detect a change that provides clear mechanistic insight, and this would identify which of the remaining assays should be used. To give an example, phagocytosis and motility both rely on the cytoskeleton. A compound-induced change to one of these functions would prompt an investigation into the effects of the compound on the other cell function.

In addition to considering the knowledge gain from each assay, the experimental hierarchy should consider several practical aspects such as cost, time, and availability of the compound. Therefore, the screening strategy must incorporate a cost-effective, timeeffective, and compound-conserving workflow that would make for an appealing tool in drug discovery. The cost of drug discovery is a hurdle in the field, and creating this workflow aims to facilitate the testing of compounds for immunomodulatory effects. As such, the process needs to be straightforward and enable a quick and clear decision-making process to identify bioactive compounds and highlight the best route of investigation to pursue to determine the mode of action. 


\subsection{Aims and objectives}

To develop a screening strategy

Specifically:

1. Review assay sensitivity, informativeness, and efficiency by testing several compounds.

2. Generate a data set which represents changes to cell activities induced by a variety of compounds.

3. Use this data, and acquired practical experience, to design a screening strategy that suits drug discovery. 


\subsection{Results}

\subsubsection{The Screen-building compound set}

After optimising several in vitro assays to determine a compound's ability to alter immune cell function, the next step in the process was to further evaluate the foundation of the drug screen, i.e. ensure the assays and selected outputs were useful in identifying immunomodulatory potential when compounds, other than the positive control, are tested. To address this, every positive control used across the assays was assembled into a set which was run through each assay. Because the mechanisms of action of each of these compounds was fairly well understood and varied between compounds, these assays would generate a sample data set representative of how a wide range of test compounds might look after being run through the screen.

These data would also be used to build the screening strategy. Each compound would have an effect in at least one of the assays of the screen, and observations of effects seen in other assays would work towards identifying relationships (reciprocal changes) between assay outputs. This would identify assays that should come early in the strategy to inform which subsequent assays should be run.

Given that treatment concentration is an important factor in inducing detectable changes in each assay - as observed during the assay optimisation process - the same concentrations used during assay optimisation were used in this chapter. Briefly, ten-fold dilutions between $1 \mathrm{nM}-100 \mu \mathrm{M}$ were used for most compounds. Glatiramer acetate has previously been used in our lab group ( $100-200 \mu \mathrm{g} / \mathrm{mL})$, so a four-fold dilution concentration range between 1.56 - $1600 \mu \mathrm{g} / \mathrm{mL}$ was chosen[115]. Similarly, Clozapine and Risperidone have been previously tested in our lab group through similar assays; therefore these compounds were tested with a narrower concentration range: two-fold dilutions between $12.5-400 \mu \mathrm{M}[94,97]$. Clozapine, an atypical antipsychotic agent, was included in this compound set as it has documented immunomodulatory properties, including reducing IL-12 production by LPS-stimulated macrophages. Data from testing clozapine would provide information to both build the screen and to re-assess the differences observed between RAW 264.7 and BMDM activation, an issue identified in the previous chapter. A summary of the compounds used in Chapter 3 and this chapter follows (Table 4.1). 
Table 4.1. Screen building compound set: expected activities and concentrations used.

\begin{tabular}{|c|c|c|c|c|}
\hline Drug & Target/activity & $\begin{array}{l}\text { Assays with } \\
\text { expected activity }\end{array}$ & $\begin{array}{l}\text { Conc range } \\
\text { tested }\end{array}$ & Ref. \\
\hline $\begin{array}{l}\text { Latrunculin A } \\
\text { (LatA) }\end{array}$ & Actin depolymeriser & $\begin{array}{l}\text { Phagocytosis and cell } \\
\text { motility inhibition }\end{array}$ & $1 \mathrm{~nm}-100 \mu \mathrm{M}$ & {$[101,104]$} \\
\hline Colchicine & Microtubule destabiliser & $\begin{array}{l}\text { Degranulation and cell } \\
\text { motility inhibition }\end{array}$ & $1 \mathrm{~nm}-100 \mu \mathrm{M}$ & {$[67,88,103]$} \\
\hline Paclitaxel & Microtubule stabiliser & $\begin{array}{l}\text { Cell proliferation } \\
\text { inhibition, inhibits } \\
\text { degranulation }\end{array}$ & $1 \mathrm{~nm}-100 \mu \mathrm{M}$ & $\begin{array}{l}{[94,143-} \\
145]\end{array}$ \\
\hline Risperidone & $\begin{array}{l}\text { Atypical antipsychotic } \\
\text { with anti-inflammatory } \\
\text { activity }\end{array}$ & $\begin{array}{l}\text { Altered macrophage } \\
\text { cytokine production }\end{array}$ & $12.5-400 \mu \mathrm{M}$ & {$[94,96]$} \\
\hline Clozapine & $\begin{array}{l}\text { Atypical antipsychotic } \\
\text { with anti-inflammatory } \\
\text { activity }\end{array}$ & $\begin{array}{l}\text { Altered macrophage } \\
\text { cytokine production }\end{array}$ & $12.5-400 \mu \mathrm{M}$ & {$[94,97,146]$} \\
\hline $\begin{array}{l}\text { Glatiramer } \\
\text { acetate (GA) }\end{array}$ & $\begin{array}{l}\text { Peptide, with anti- } \\
\text { inflammatory activity in } \\
\text { multiple sclerosis }\end{array}$ & $\begin{array}{l}\text { Altered macrophage and } \\
\text { T cell cytokines }\end{array}$ & $\begin{array}{l}1.56- \\
1600 \mu \mathrm{g} / \mathrm{mL}\end{array}$ & $\begin{array}{l}{[68,70,112-} \\
115]\end{array}$ \\
\hline Cycloheximide & $\begin{array}{l}\text { Protein synthesis } \\
\text { inhibitor (translation) }\end{array}$ & $\begin{array}{l}\text { Inhibition of cell } \\
\text { proliferation, cytokine, } \\
\text { and antibody production }\end{array}$ & $1 \mathrm{~nm}-100 \mu \mathrm{M}$ & {$[147,148]$} \\
\hline
\end{tabular}

Because many of these studies used a targeted approach to determine bioactivity, it was not obvious how these compounds would behave in our immunological assays. Thus, in this chapter, novel information about the immunomodulatory activities of these known compounds was collected. For example, at the time of conducting these experiments it was not known how clozapine might alter B cell activity, nor how risperidone might impact phagocytosis. For this reason, data from testing compounds are presented by assay to appreciate these effects, and a summary and interpretation of effects are presented towards the end of the section. 


\subsubsection{Screen-building set compounds: effects on macrophage activation}

Macrophages primed with IFN- $\gamma$ and stimulated with LPS become classically activated, producing NO, TNF $\alpha$, IL-12 and IL-10. In the presence of test compounds, these cells can have altered cytokine production, suggesting changes to inflammatory state. Risperidone, a positive control for altering macrophage activation, causes a change in cytokine production which is most evident at $200 \mu \mathrm{M}$ (Figure 4.1, grey box). Risperidone caused an increase in IL-12, and a moderate increase in IL-10. The pro-inflammatory TNF $\alpha$ is reduced at this concentration, and a small change in NO is also observed. It is important to note that the decrease in the measured outputs at $400 \mu \mathrm{M}$ is likely due to a loss in viability, as indicated by MTT data. Clozapine also demonstrates a modulatory effect on macrophage cytokines with IL-12 concentrations decreasing from the lowest concentration tested of $12.5 \mu \mathrm{M}$; IL-10, NO and TNF $\alpha$ are not remarkably altered. Glatiramer acetate also has effects on macrophage activation state, and this is most apparent at the relatively high concentration of $400 \mu \mathrm{g} / \mathrm{mL}$ which caused an increase in IL-12 production.

Cycloheximide decreases all outputs measured in this assay: both IL-10 and IL-12 decrease, alongside TNF $\alpha$ and NO to a lesser extent. Interestingly, at $0.1 \mu \mathrm{M}$, before cell viability is detectably decreased, IL-12 levels in the supernatant rise dramatically. Latrunculin A (LatA) has negative effects on cell viability which is reflected by the IL-12, TNF $\alpha$ and NO production by the cells. In contrast, IL-10 from LatA-treated macrophages increases dramatically at concentrations above $0.01 \mu \mathrm{M}$. This occurs whilst cell viability is decreasing.

The two microtubule (MT) targeting drugs, paclitaxel and colchicine, had different effects on macrophage activation in this assay. Paclitaxel, the MT stabiliser, had little effect on IL-12 or NO release but led to a modest reduction in IL-10 production. The most evident effect of this compound was on TNF $\alpha$ production, which was reduced by $50 \%$ with $1 \mu \mathrm{M}$ paclitaxel. At $10 \mu \mathrm{M}$ paclitaxel, the TNF $\alpha$ levels increased slightly, which may reflect the LPS-mimicry of paclitaxel[95]. In contrast to paclitaxel, the MT destabiliser colchicine caused a dramatic change in IL-10 and IL-12 production whilst NO and TNF $\alpha$ were only modestly changed. Specifically, IL-10 decreased with $0.1 \mu \mathrm{M}$ Colchicine and above, whereas at the same concentrations, IL-12 levels increased by almost two-fold. 

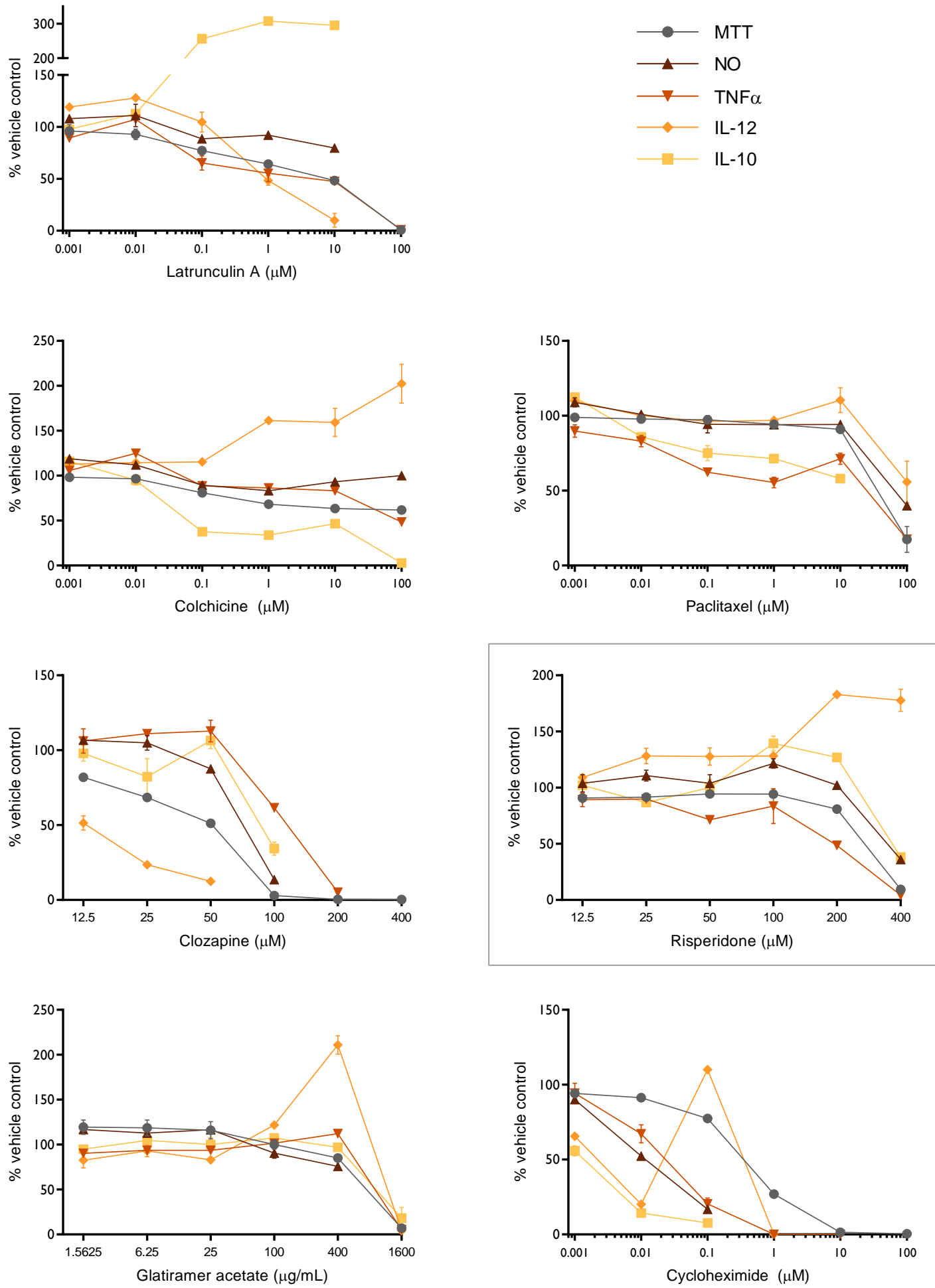

Figure 4.1. The effects of screen-building set compounds on macrophage activation.

50,000 RAW 264.7 cells were seeded into wells of a 96-well plate and primed with $20 \mathrm{U} / \mathrm{mL}$ IFN $\gamma$ for $18 \mathrm{~h}$. Compounds or vehicle treatments were added to wells alongside $200 \mathrm{ng} / \mathrm{mL}$ LPS. After $24 \mathrm{~h}$, supernatants were harvested for ELISA and Griess analyses, and cells subjected to MTT assay to assess viability. Results are means and SEM from one experiment conducted in duplicate; Risperidone data presented here are the same as presented in Figure 3.3. 
The use of the RAW264.7 cell line in this assay assumes that this cell line will accurately predict an immunomodulatory effect on primary macrophages. This was shown not to be the case in the previous chapter after identifying that risperidone induced different changes during RAW 264.7 and BMDM activation. The macrophage activation data from treated RAW 264.7 cells (Figure 4.1) were compared to data from treated and activated BMDM (see appendix section 9.4.1, pg 205) to assess if the discrepancy between the two cell sources was specific to risperidone treatments or was characteristic of the cell line.

In contrast to cytokines measured, there was very little impact on NO production by activated macrophages treated with this compound set, and the same was seen with BMDM cells. TNF $\alpha$ data from BMDM was limited, though where observed the changes were similar between the two cell types. However, changes to IL-10 and IL-12 production differed between RAW 264.7 cells and BMDM with most of the compounds. Compound effects on IL-12 and IL-10 production were similar between the two cell types when treated with LatA and Paclitaxel. Cycloheximide inhibited IL-10 production to a greater extent in RAW 264.7 cells than in BMDM. However, where a decrease in IL-12 was seen with RAW 264.7 cells treated with $0.001 \mu$ M cycloheximide, a slight increase in IL-12 was seen in BMDM. With colchicine treatment, IL-10 and IL-12 production responds in opposite ways between the two cell types. IL-10 and IL-12 production by BMDM treated with glatiramer acetate, clozapine and risperidone were vastly different to the changes seen in RAW 264.7 cells. Treatment with $400 \mu \mathrm{g} / \mathrm{mL}$ Glatiramer acetate sees a spike in IL-12 production in RAW 264.7 cells, but an IL-10 spike instead from BMDM. Clozapine suppresses IL-12 production in the cell line, but enhances IL-10 production in BMDM; the changes induced by Risperidone reflect previous observations.

Whilst changes to NO and TNF $\alpha$ production in RAW 264.7 cells reflect changes in BMDM, the changes to IL-10 and IL-12 production induced by compounds differ between the two cell populations. Though primary BMDM are a better model to assess immunomodulation during macrophage activation, their culture is resource-heavy and time-consuming and require animals as a source of cells. The compromise is to use the more convenient RAW 264.7 cell line and acknowledge that changes to IL-10 and IL-12 only can demonstrate a compound's ability to alter cytokine production; specific changes to these cytokines do not represent immunomodulation seen in primary macrophages which are more representative of in vivo macrophages. Once compounds are screened, and activity 
is seen against activated RAW 264.7 cells, the compound should be tested against activated BMDM to determine specific changes to cytokine production.

To summarise, each compound uniquely altered macrophage activation, causing distinct changes to cytokine production; NO was not altered by any compound tested. Interestingly, MT targeting drugs did not alter macrophages in similar ways. Unsurprisingly, protein synthesis inhibition with cycloheximide suppressed cytokine production. Although the specific changes to IL-10 and IL-12 production by treated RAW 264.7 cells appear not to be representative of the changes that would be seen in vivo, they afford the opportunity to assess macrophage activation modulation in a medium-throughput manner.

\subsubsection{Screen-building set compounds: effects on macrophage viability}

When unactivated, RAW 264.7 cells proliferate in culture during the assay period; however, when activated with IFN $\gamma$ and LPS, RAW 264.7 cells are non-proliferative. By comparing MTT data of cells treated with compounds between these two states, antiproliferative compounds can be identified. MTT measures metabolic activity from which viability is inferred. For this experiment, MTT data from compound treatments are presented as a percentage of vehicle-treated MTT data, with graphs comparing unactivated (proliferating) cells to activated (non-proliferating) cells.

As mentioned in the previous chapter, paclitaxel (Figure 4.2, grey box) is an antiproliferative drug. In this assay system, the unactivated cells (dotted line) have decreased viability compared to activated cells. At concentrations between $0.01-1 \mu \mathrm{M}$ paclitaxel, the MTT response steadily decreased to around $20 \%$ of vehicle-treated cells. Between these concentrations, activated cells are unaffected, retaining the same viability of vehicle-treated cells. At $10 \mu \mathrm{M}$, a sharp increase in unactivated cell viability occurs - indicating that the untreated cells are metabolically active - and this activity may be due to the reported LPS mimicry of paclitaxel overriding the cytostatic or -toxic effects of the drug. Beyond $10 \mu \mathrm{M}$ paclitaxel, both cell states succumb to the drug. Like with paclitaxel, when proliferating cells are treated with cycloheximide, their viability is affected at lower concentrations than that of activated cells, indicated by the left shift of unactivated cell viability (dotted) compared to activated (solid). Similarly, proliferating cells treated with $0.1-100 \mu \mathrm{M}$ 
colchicine also have reduced viability compared to non-proliferating cells. In contrast to the effects of paclitaxel, colchicine, and cycloheximide, increasing concentrations of LatA decreased cell viability regardless of activation state.

Risperidone, clozapine, and glatiramer acetate showed similar patterns of effect on proliferating and non-proliferating RAW 264.7 cells. Risperidone abolished metabolic activity in both cell states at $400 \mu \mathrm{M}$, and a similar effect was seen at $100 \mu \mathrm{M}$ for clozapine. Glatiramer acetate was tolerated by both activated and unactivated RAW 264.7 cells at concentrations up to $400 \mu \mathrm{g} / \mathrm{mL}$ before both proliferating and non-proliferating cells succumbed to drug. Together these results have identified several compounds that preferentially target proliferating cells (especially paclitaxel and colchicine), while also identifying the concentration at which compounds become toxic to RAW 264.7 cells.

This assay identifies compounds that have strong effects on cellular proliferation, as seen with mid-concentrations of paclitaxel and colchicine. Cells treated with these compounds have greatly reduced viability when proliferating, but are relatively viable when nonproliferating. This suggests that these compounds induce cell death through mechanisms strongly associated with proliferation. Indeed, microtubule rearrangements are important for cell division. Although cycloheximide also negatively affects proliferating cells, it also affects non-proliferative yet active cells. This suggests its target is not specifically antiproliferative when inducing cell death, however proliferating cells are more sensitive to protein synthesis inhibition. Similarly, clozapine reduces the viability of activated macrophages by a greater extent than unactivated macrophages. This suggests that the target of clozapine is more active, essential, or present, during macrophage activation. Other compounds alter cell viability similarly between proliferative and non-proliferative cell states suggesting these compounds act through mechanisms that are not specific to proliferative state. This assay offers a wealth of information on compound activity that extend beyond cytokine and NO production with the inclusion of the unstimulated macrophage condition. 

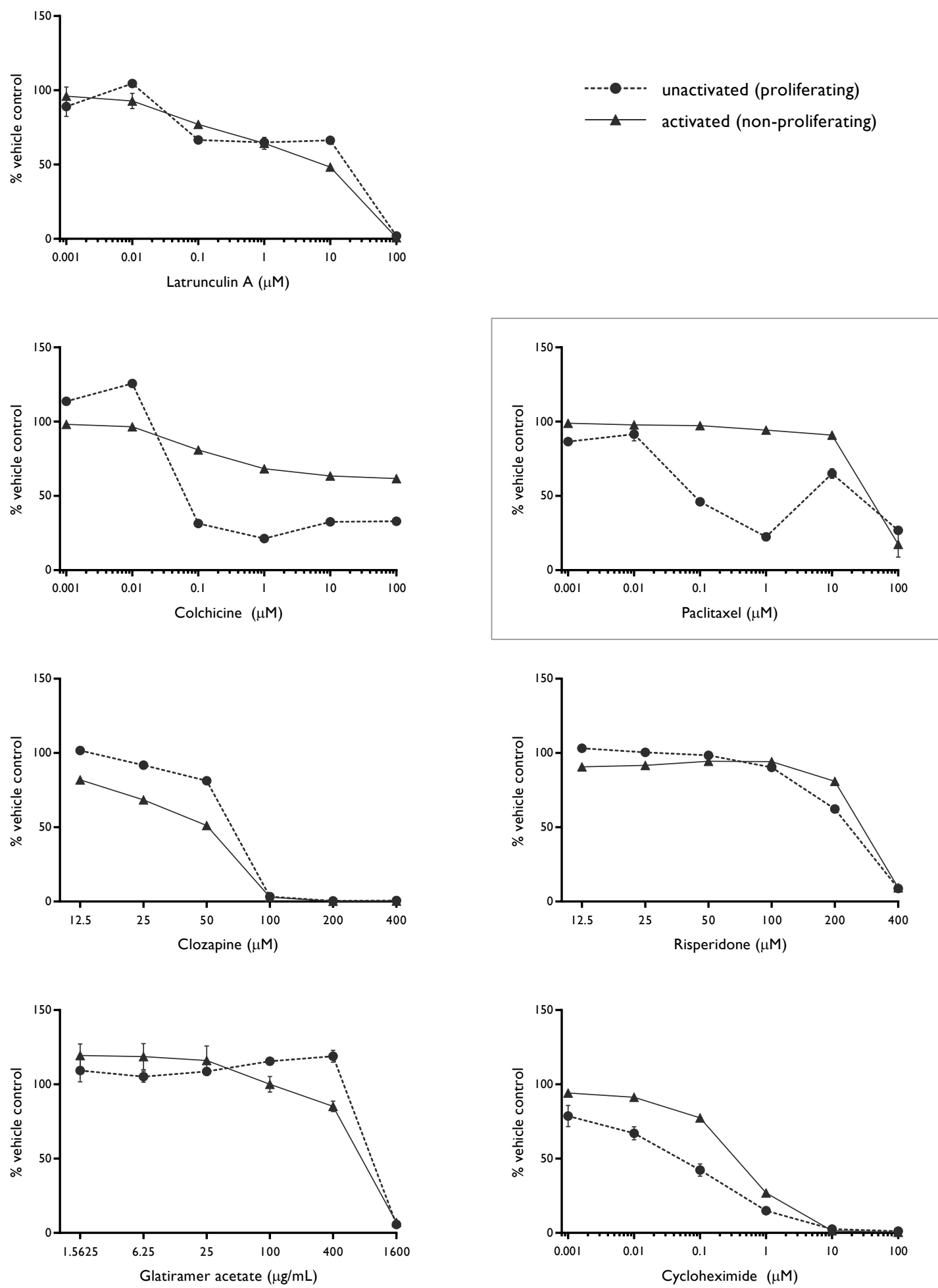

Figure 4.2. RAW 264.7 viability differences due to compound treatment and proliferative states. 50,000 RAW 264.7 cells were seeded into wells of a 96-well plate and incubated for $18 \mathrm{~h}$. Indicated treatments were added alongside vehicle controls, and incubated for a further $24 \mathrm{~h}$. MTT was added to wells to assess viability. Viability data of treated proliferating, unstimulated cells (dotted) were graphed with viability of treated activated, non-proliferating cells (solid). Data are means and SEM from one experiment conducted in duplicate; data for cells treated with Paclitaxel are the same as presented in Figure 3.2, BFigure 3.3. 


\subsubsection{Screen-building set compounds: effects on macrophage phagocytosis}

This assay evaluates the ability of RAW 264.7 cells to phagocytose particles after drug treatment, and their ability to produce cytokines during this process. Given the critical role for actin polymerisation during phagocytosis, LatA serves as the positive control (Figure 4.3, grey box). At concentrations where the positive control does not significantly alter cell viability, $10 \mu \mathrm{M}$ and below, phagocytosis was heavily impaired. TNF $\alpha$ production was also inhibited by latrunculin treatment whereas IL-6 production increased to almost 150\% of vehicle-treated cells, though this dropped with $10 \mu \mathrm{M}$ LatA.

Phagocytic ability was also affected by colchicine at concentrations of $0.1 \mu \mathrm{M}$ and above, though this only decreased to $70 \%$ of control. TNF $\alpha$ production was also inhibited by higher concentrations of colchicine, but IL-6 appeared to fluctuate, with a sharp increase detected at $1 \mu \mathrm{M}$. Paclitaxel had little effect on cytokine production in this assay but led to a striking reduction in phagocytosis at concentrations of 1 and $10 \mu \mathrm{M}$. These data indicate that interference with MT, like actin microfilaments, disrupts phagocytosis in macrophages.

Although both phagocytosis and cytokine production were reduced by clozapine, it appears that phagocytosis was impaired to a greater extent (i.e. at drug lower concentrations) than cytokine production. Risperidone treatment inhibited TNF $\alpha$ production during this assay but only moderately inhibited phagocytosis and IL-6. Major effects of Glatiramer acetate during this assay were seen when cells were treated with $400 \mu \mathrm{g} / \mathrm{mL}$, where TNF $\alpha$ production is abolished, and phagocytosis and IL-6 were reduced to 50\% vehicle control. Cycloheximide had no effect on phagocytosis, but impaired cytokine production even at low concentrations.

To summarise, phagocytosis can be reduced by altering the cytoskeletal dynamics of macrophages, however it is not the only way to alter this process. Atypical antipsychotics acting through dopamine receptors can also inhibit phagocytosis, as can glatiramer acetate. Unsurprisingly, protein synthesis inhibition has little effect on phagocytosis, but it suppresses cytokine production, similarly to what was seen in the macrophage activation assay. TNF $\alpha$ alteration, e.g. by paclitaxel, seen during the macrophage activation assay was not observed in this assay, which may be due to the different durations of the two experiments. 

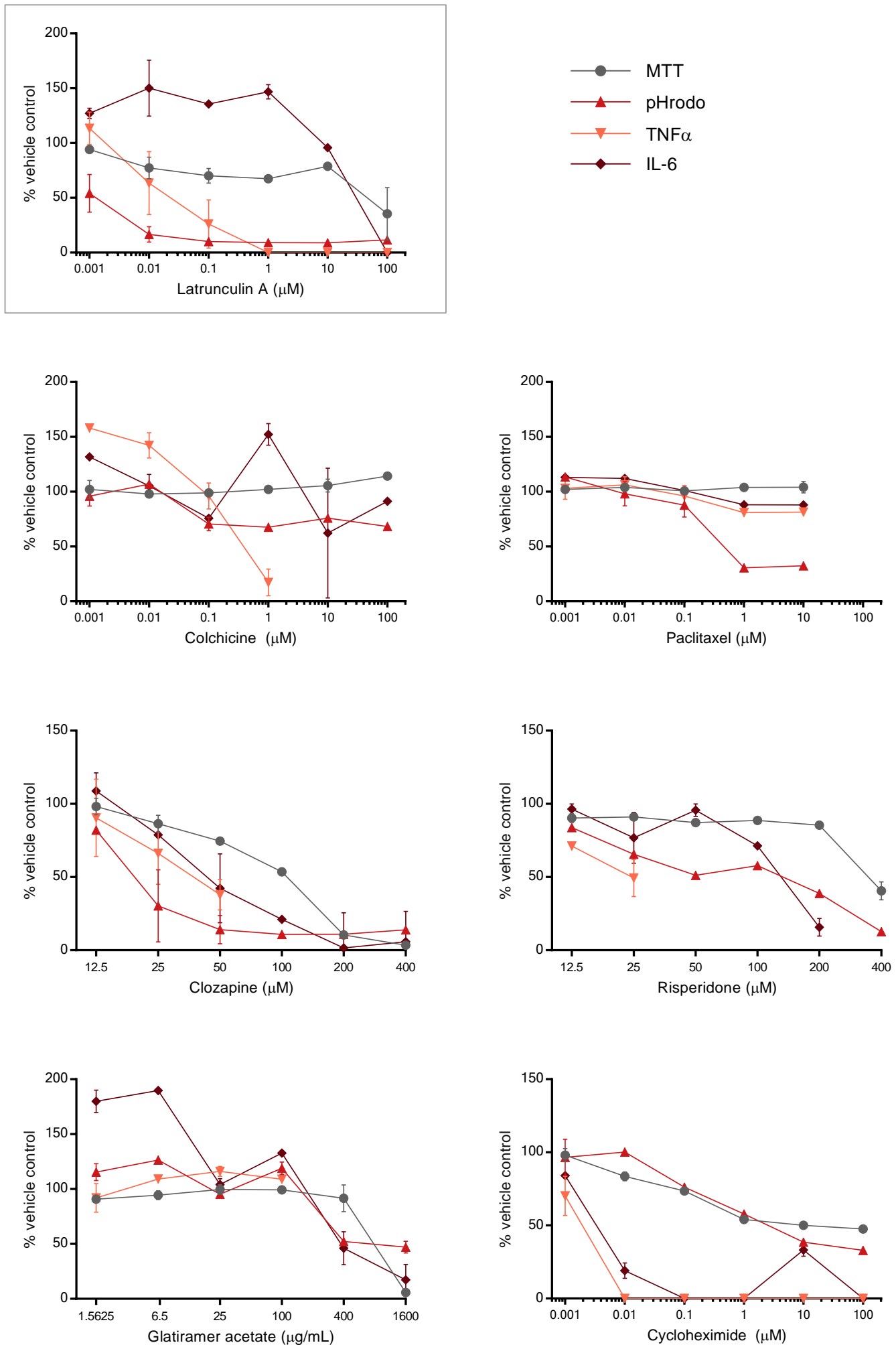

Figure 4.3. The effects of screen-building set compounds on macrophage phagocytosis.

100,000 RAW264.7 cells were seeded into wells of a 96-well plate alongside indicated treatments and vehicle equivalents. After this $4 \mathrm{~h}$ pre-treatment, $25 \mu \mathrm{g} / \mathrm{mL}$ pHrodo bioparticles were added to wells alongside further treatment to maintain concentration. After a further $4 \mathrm{~h}$, supernatants were collected for cytokine analysis by ELISA, and cells were subjected to flow cytometry to assess phagocytosis. MTT data were collected from cells seeded and pre-treated alongside the previously mentioned experiment. Data are means and SEM from one experiment with treatments conducted in duplicate; Latrunculin A data presented here are the same as presented in Figure 3.6. 
During this experiment, the need to run two plates for this assay was deemed inefficient. One set of treated cells were used to assess phagocytosis, and a parallel set was used to assess cell viability by MTT assay. A subsequent experiment investigated the use of fluorescent viability dye as a method to assess cell viability as an alternative to MTT (see appendix 9.4.2, pg 207). The zombie dye assesses viability through membrane exclusion as live cells have intact membranes that exclude the dye from cells; dead cells lose membrane integrity allowing the dye to enter. It is a fluorescent dye that is measured using flow cytometry which could be assessed simultaneously with phagocytosis assessment which would mean only one set of treated cells would be needed to complete the assay.

When comparing viability assessment methods, it appeared zombie dye data were less informative: they rarely showed altered viability by compound treatments. For LatA and cycloheximide treatment, cell viability had decreased when assessed by MTT and this was not reflected in the zombie dye data. The differences can be explained through the ways the two methods assess viability: zombie dyes are membrane impermeable, and only identify dying/dead cells after membrane integrity is lost whereas MTT viability is determined by cell metabolism. Although both techniques have limitations, for this short assay it was deemed more informative to acknowledge a change in cell metabolism rather than membrane integrity. If phagocytosis decreased alongside diminished metabolic activity, the change in phagocytic activity could be an indirect effect of compound treatment or alternatively, the changes to these processes could be through a shared mechanism. For this reason, the MTT assay was deemed a more informative method by which to determine cell viability for this assay. The same was deemed for the degranulation assay which has a similar assay duration. 


\subsubsection{Screen-building set compounds: effects on macrophage motility}

In this assay, the ability of compound-treated BMDM to close a "wound" is used as an indicator of cell motility. Cell viability is also monitored to determine if a lack of wound closure was due to diminished cell viability. Data are presented as percentages of vehicle control: at $100 \%$, the viability or motility of cells is equivalent between compound- and vehicle-treated cells. At $0 \%$, motility of treated cells is not observed (wound has not changed size), and when this measure becomes negative, it indicates the wound has increased from its original size in treated wells.

LatA, the positive control, completely inhibited wound closure: at concentrations of 0.1 $\mu \mathrm{M}$ and above, $0 \%$ motility was detected and was not an effect of cells dying (Figure 4.4, grey box). Colchicine also significantly impaired wound closure. At the lowest concentration tested, $0.001 \mu \mathrm{M}$, motility was only around $15 \%$ of vehicle-treated cells. At the next highest concentration, the wound became larger than its original size. Higher concentrations are not shown as cell morphology changed to a point where cells were no longer adhered to the plate, a known effect of MT destabilisation[149-151]. Paclitaxel also inhibited cell motility, though the effects were not as dramatic as either LatA or colchicine. Interestingly, lower concentrations of paclitaxel inhibited cell motility to a greater extent than higher concentrations of the microtubule stabiliser.

Clozapine, risperidone and glatiramer acetate all inhibited cell motility to various extents. Risperidone showed its greatest effect in this assay at $200 \mu \mathrm{M}$, clozapine at lower concentrations of 12.5 and $50 \mu \mathrm{M}$, and glatiramer acetate at 100 and $400 \mu \mathrm{g} / \mathrm{mL}$. Finally, inhibiting protein synthesis with cycloheximide also inhibited cell motility, and the effect was most evident between $0.001-0.1 \mu \mathrm{M}$ cycloheximide. At concentrations above those mentioned, motility appears to be indirectly inhibited due to reduced cell viability.

Much like in the previous assays, some of these data are not surprising. Altering MTs impaired cell motility, explained by the role of the cytoskeleton in cell motility. However, the suppression of motility with clozapine, risperidone and glatiramer acetate are interesting, as is cycloheximide's suppression of this cell activity. 

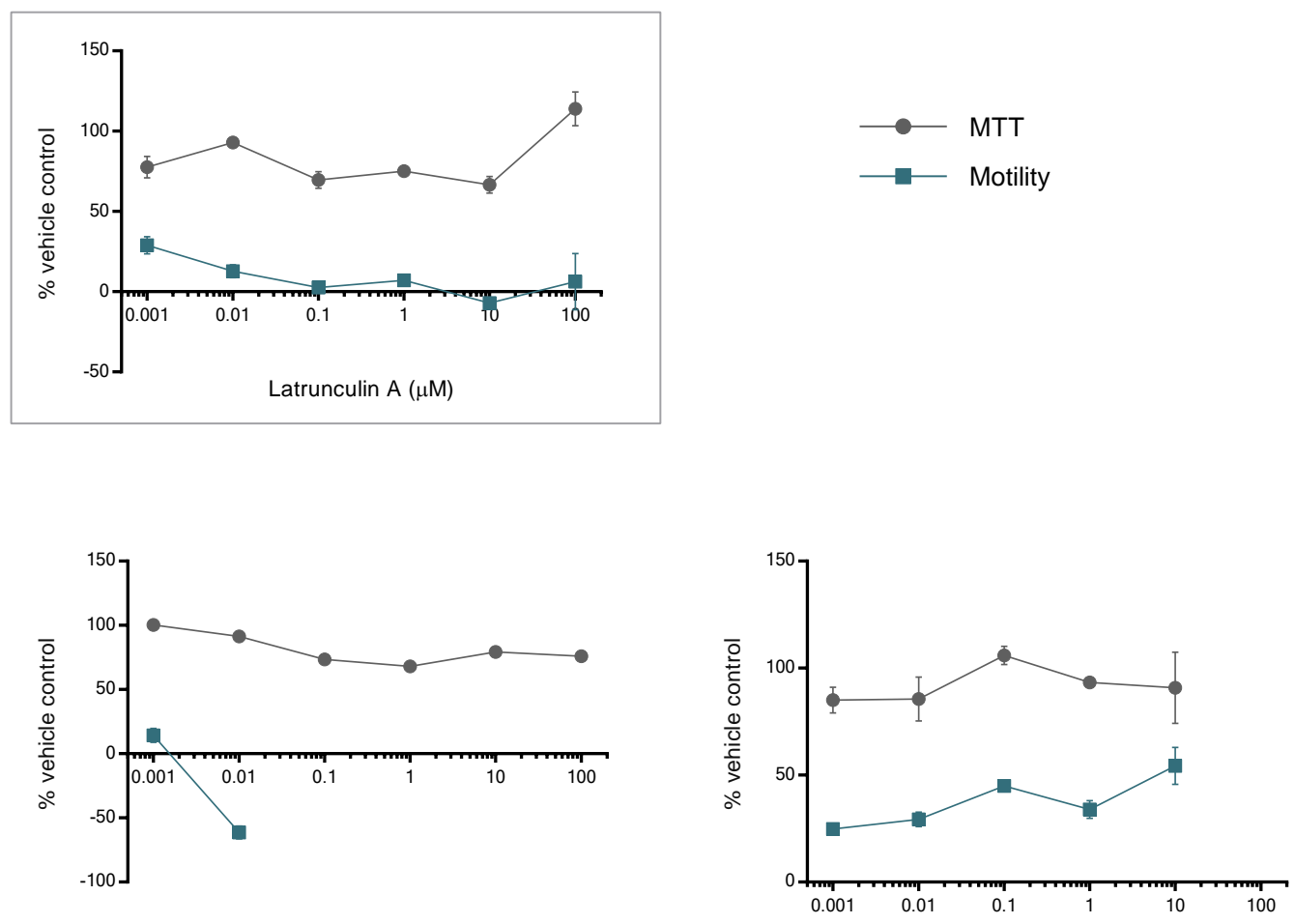

Colchicine $(\mu \mathrm{M})$

Paclitaxel $(\mu \mathrm{M})$
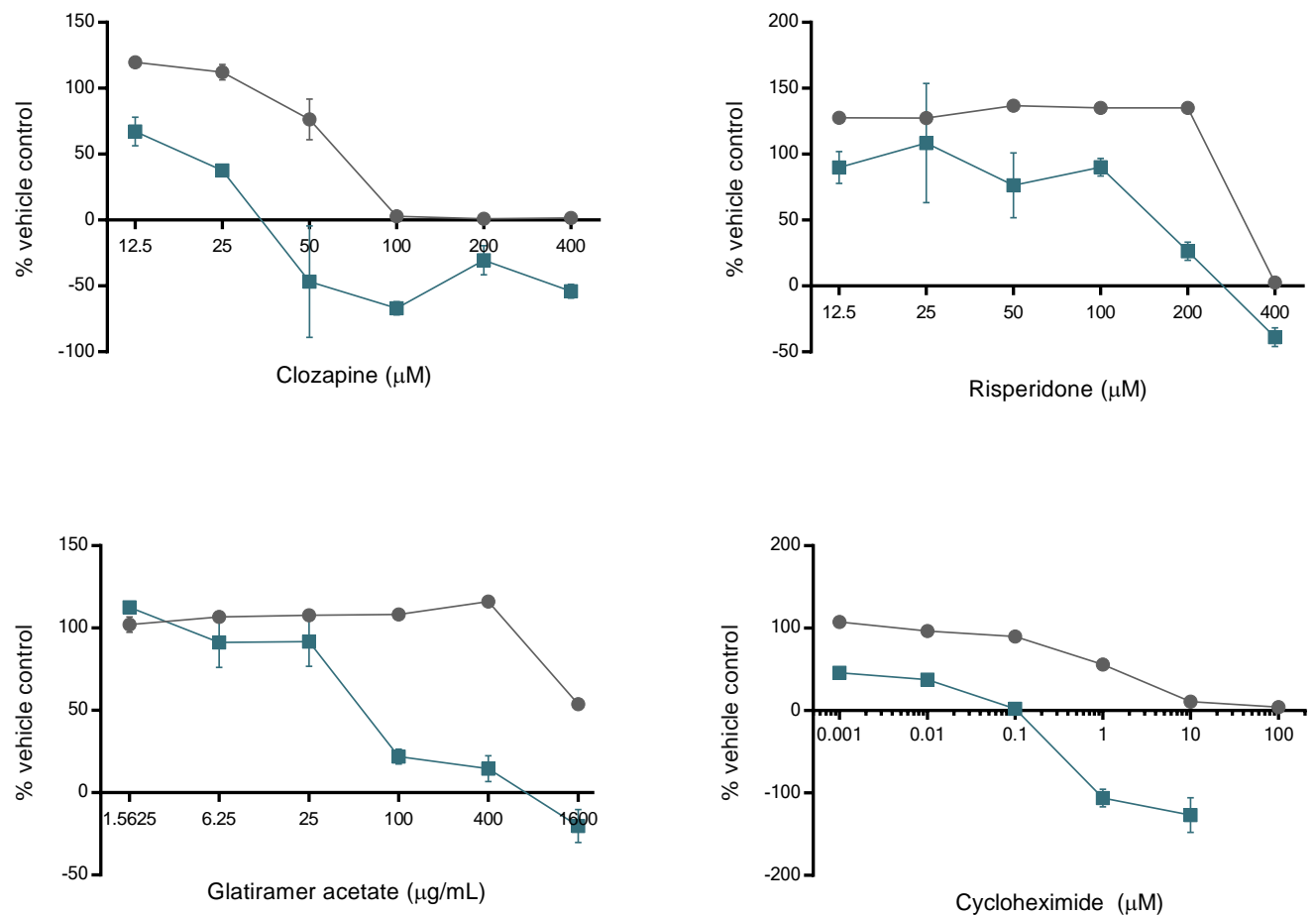

Figure 4.4. The effects of screen-building set compounds on macrophage motility

120,000 BMDM were seeded into ORIS plates and allowed to settle overnight. Plugs were removed and wound sizes were measured. Indicated treatments or equivalent vehicles were added to wells and incubated for three days. Final wound sizes were measured before cells were subjected to MTT assay to assess cell viability (black). Motility was calculated by comparing wound size change by treatments compared to that of vehicles, with negative values indicating final wound size increasing from original size. Wound closure in vehicle-treated wells averaged $19.21 \%(\mathrm{SD}=1.11 \%)$. Data are means and SEM from one experiment conducted in duplicate; LatA data presented here are the same as presented in Figure 3.9. 


\subsubsection{Screen-building set compounds: effects on helper $T$ cell activation}

Upon activation, T cells produce cytokines which can infer which Th subset they have differentiated into. This was observed with glatiramer acetate, the positive control, which caused small decreases in IL-10, and small increases in IFN $\gamma$ (Figure 4.5, grey box). Additionally, IL-17a was elevated across all concentrations tested and IL-4 decreased when lower concentrations of compound were present, only increasing with $400 \mu \mathrm{g} / \mathrm{mL}$ drug. Concentrations of glatiramer acetate above $400 \mu \mathrm{g} / \mathrm{ml}$ abolished cytokine production due to the effect on viability.

Although LatA treatment induced high levels of IL-4 compared to other assay outputs, cell viability was very poor. Even at the lowest tested concentration of $0.001 \mu \mathrm{M}$, cell viability was only $10 \%$, and this negative effect on viability was likely responsible for the decrease in other cytokine levels. The lowest concentration of colchicine (i.e. $0.001 \mu \mathrm{M}$ ), did not impair cell viability but, like LatA, IL-4 was increased as was IFN $\gamma$. In contrast, IL-17A and IL-10 both decreased. Paclitaxel modestly induced IL-4 and IL-17A while IL-10 levels decreased, and IFN $\gamma$ remained unchanged. Altogether, these cytoskeletal modifiers each mediated a distinct pattern of cytokine modulation.

At most concentrations of clozapine, changes in the cytokine levels correlated to cellular viability. At the lowest concentration (i.e. $12.5 \mu \mathrm{M}$ ), where viability was reduced to $\sim 60 \%$, IL-4 andIL-10 were elevated and IFN $\gamma$ levels were reduced whilst IL-17A was unaffected. Similarly, risperidone also affected splenocyte viability. However, at $12.5 \mu \mathrm{M}$ risperidone, viability was not affected and IL-10 and IL-17A were elevated while IL-4 and IFN $\gamma$ levels were unchanged.

Cycloheximide also effected cell viability at the lowest tested concentration: viability was only $70 \%$ of the vehicle control. At this concentration of cycloheximide, IL-10 and IL17A decreased, and this decrease was likely linked to the general reduction in cell viability. IL-4 levels remained similar to vehicle-treated cells, and IFN $\gamma$ levels were slightly increased in the presence of this protein synthesis inhibitor. 

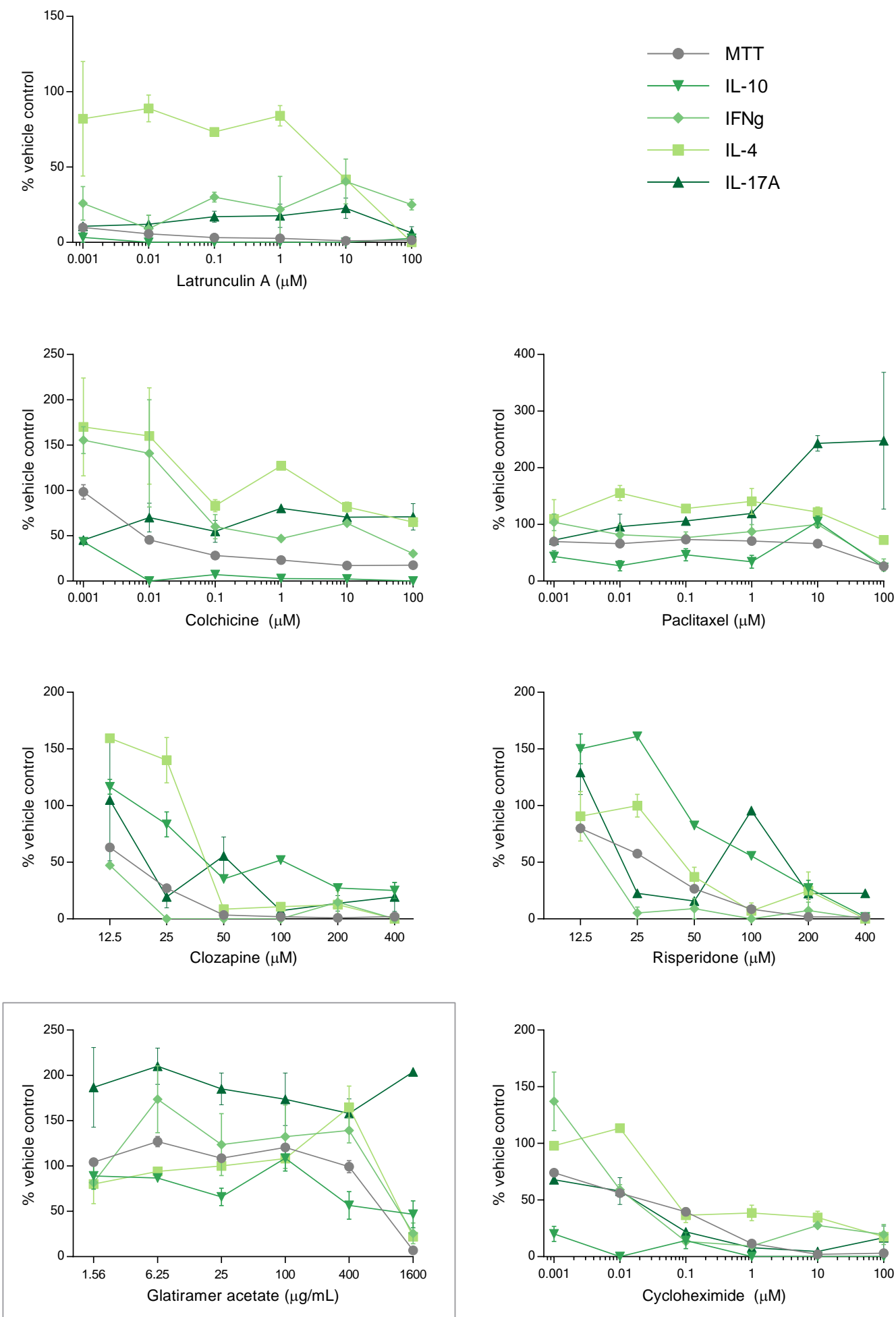

Figure 4.5. The effects of screen-building set compounds on helper $\mathbf{T}$ cell activity $1 \times 10^{6}$ splenocytes were seeded into 96-well plates and stimulated with $1 \mu \mathrm{g} / \mathrm{mL}$ ConA alongside indicated treatments and vehicle equivalents. After $48 \mathrm{~h}$, supernatants were analysed by ELISA for cytokines, and cell viability was assessed by MTT. Data are means and SEM from one experiment conducted in duplicate; Glatiramer acetate data presented here are the same as presented in Figure 3.13. 
A common trend throughout these assays is the observation that a wide variety of compounds can alter immune cell function, and this was also seen in the Helper $\mathrm{T}$ cell assay. All compounds altered the levels of cytokines produced upon activation, though in different ways. The two antipsychotics had different effects on Th cytokine production, with risperidone strongly inducing IL-10 production whilst clozapine appeared to promote IL-4 production. The MT targeting drugs also induced different changes to Th cytokines.

\subsubsection{Screen-building set compounds: effects on splenocyte viability}

Splenocytes undergo a large change in activity when stimulated with ConA as many cellular processes are initiated, including proliferation. Differences between stimulated and unstimulated splenocyte viability may be useful for deepening the understanding of a compound's immunomodulatory properties and can identify compounds that affect $\mathrm{T}$ cells undergoing activation. In this experiment, splenocytes were treated with compounds and were stimulated with ConA or remained unstimulated (Figure 4.6). These data were generated during the Helper T cell activation assay but are presented separately for clarity.

In this assay, activated splenocytes were found to be sensitive to the actin depolymeriser LatA, with viability dropping to $10 \%$ of vehicle control at the lowest tested concentration of $0.001 \mu \mathrm{M}$. Unstimulated splenocytes were also adversely affected by this compound, with the lowest concentration resulting in around $75 \%$ viability. Splenocytes stimulated in the presence of colchicine also had decreased viability compared to their unstimulated counterparts, and while this change is moderate, similar results were observed when cells were treated with paclitaxel, the microtubule stabiliser.

The two atypical antipsychotics also adversely affected cell viability, however, there were differences between the two compounds. Clozapine appeared to have a greater effect on cell viability than risperidone, and stimulation was found to enhance this effect. Glatiramer acetate was toxic to stimulated splenocytes at $1600 \mu \mathrm{g} / \mathrm{mL}$ but only reduced viability to around $50 \%$ in the absence of stimulation. Below this concentration, glatiramer acetate did not affect cell viability differently when the cells were stimulated versus unstimulated.

As expected, interfering with protein synthesis with cycloheximide affected stimulated splenocytes more than unstimulated cells. This is evident even with the lowest treatment of $0.001 \mu \mathrm{M}$ cycloheximide which brought stimulated cell viability down to around $75 \%$. 
Viability decreased quickly with increasing concentrations of cycloheximide until complete cell death was observed at $10 \mu \mathrm{M}$. Unstimulated cells became sensitive to the drug at concentrations greater than $0.1 \mu \mathrm{M}$, but viability was never fully abolished. Interestingly, at concentrations of $0.1 \mu \mathrm{M}$ or below, unstimulated splenocytes had a modest increase in cell metabolism.

By comparing the viability of unstimulated and stimulated splenocytes, compounds that reduce the viability of stimulated $\mathrm{T}$ cells interfere with $\mathrm{T}$ cell activation. This was seen with cycloheximide, LatA, and the MT-targeting compounds. These compounds target protein synthesis and the cytoskeleton which can explain why T cell activation is affected more so than resting splenocytes. This assay is suitable for identifying compounds that affect $\mathrm{T}$ cells undergoing activation. 

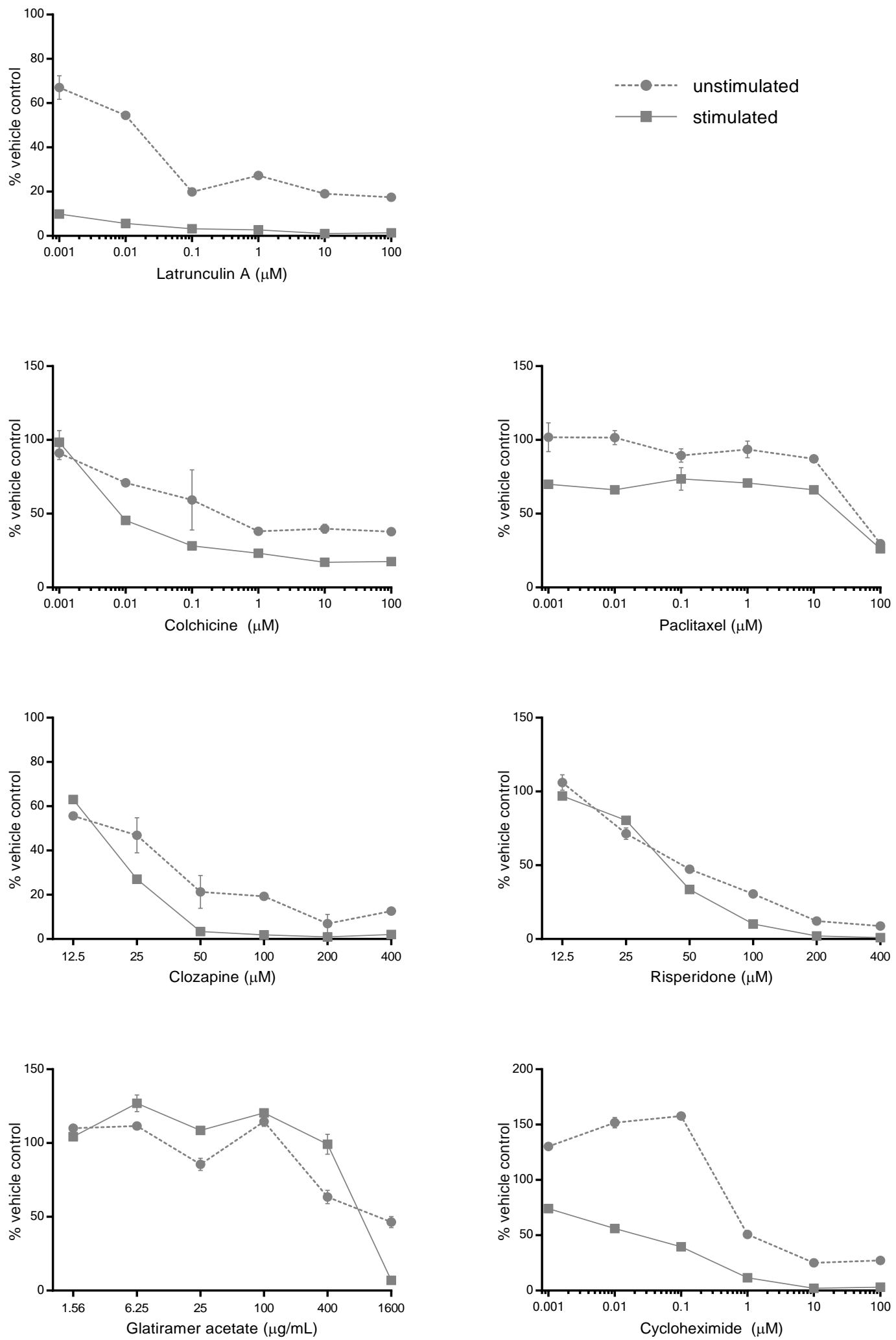

Figure 4.6. The effects of screen-building set compounds on $\mathrm{T}$ cell activation

Alongside previous experiment, $1 \times 10^{6}$ splenocytes were seeded into wells, and indicated treatments (or vehicle equivalents) were added. After $48 \mathrm{~h}$, cells were subjected to MTT assay to assess cell viability. Viability data of treated unstimulated cells (dotted) was plotted against that of stimulated cells (solid). Data are means and SEM from one experiment conducted in duplicate. Data for stimulated cells treated with Glatiramer acetate are the same as presented in Figure 3.13. 


\subsubsection{Screen-building set compounds: effects on CTL Degranulation}

The release of vesicles containing cytolytic granules is a key activity of CTLs, and this activity can be measured by monitoring the cell surface expression of CD107a. The production of IFN $\gamma$ is an additional activity of CTLs during an immune response. Colchicine, the positive control for altering degranulation, demonstrated its ability to decrease both degranulation and IFN $\gamma$ production by CTL at concentrations of $1 \mu \mathrm{M}$ and above (Figure 4.7, grey box). Although paclitaxel also modifies microtubules, it did not generate the same effects. Instead, MT-stabilising paclitaxel had only modest effects on degranulation and IFN $\gamma$ production before cell viability was adversely affected.

Actin disruption with LatA dramatically altered CTL activities while having a moderate effect on viability. With $0.001 \mu \mathrm{M}$ LatA, cell viability was reduced to $74 \%$; in contrast, IFN $\gamma$ production and degranulation dropped to $40 \%$ activity compared to vehicle controls. Cycloheximide induced a distinct pattern as it reduced IFN $\gamma$ production but not degranulation activity: at the lowest concentration of cycloheximide tested, IFN $\gamma$ production was only half that of vehicle-treated controls, and levels of this cytokine decreased in a concentration-dependent manner. Full inhibition of IFN $\gamma$ production was seen with $1 \mu \mathrm{M}$ cycloheximide at which point cell viability began to decline.

CTL activity also changed in the presence of atypical antipsychotic drugs. Clozapine's major effects were seen at $50 \mu \mathrm{M}$ where, although cell viability appeared unaffected, degranulation dropped to around 55\% and IFN $\gamma$ dropped to around 20\%. Risperidone had similar effects on IFN $\gamma$ production by CTLs where, with $50 \mu \mathrm{M}$ drug, IFN $\gamma$ production was half that of vehicle control. However, in contrast to clozapine, degranulation activity was unchanged from vehicle controls until viability began to drop at concentrations above $100 \mu \mathrm{M}$. Glatiramer acetate had little effect on degranulation by CTLs although concentrations between $1.5625-100 \mu \mathrm{g} / \mathrm{mL}$ appeared to modestly enhance IFN $\gamma$ production.

The most interesting finding from this experiment were the dissimilar ways the two MT targeting drugs alter degranulation. The other data confirmed what has been previously seen: that antipsychotics and protein inhibition mainly suppress cytokine production. 

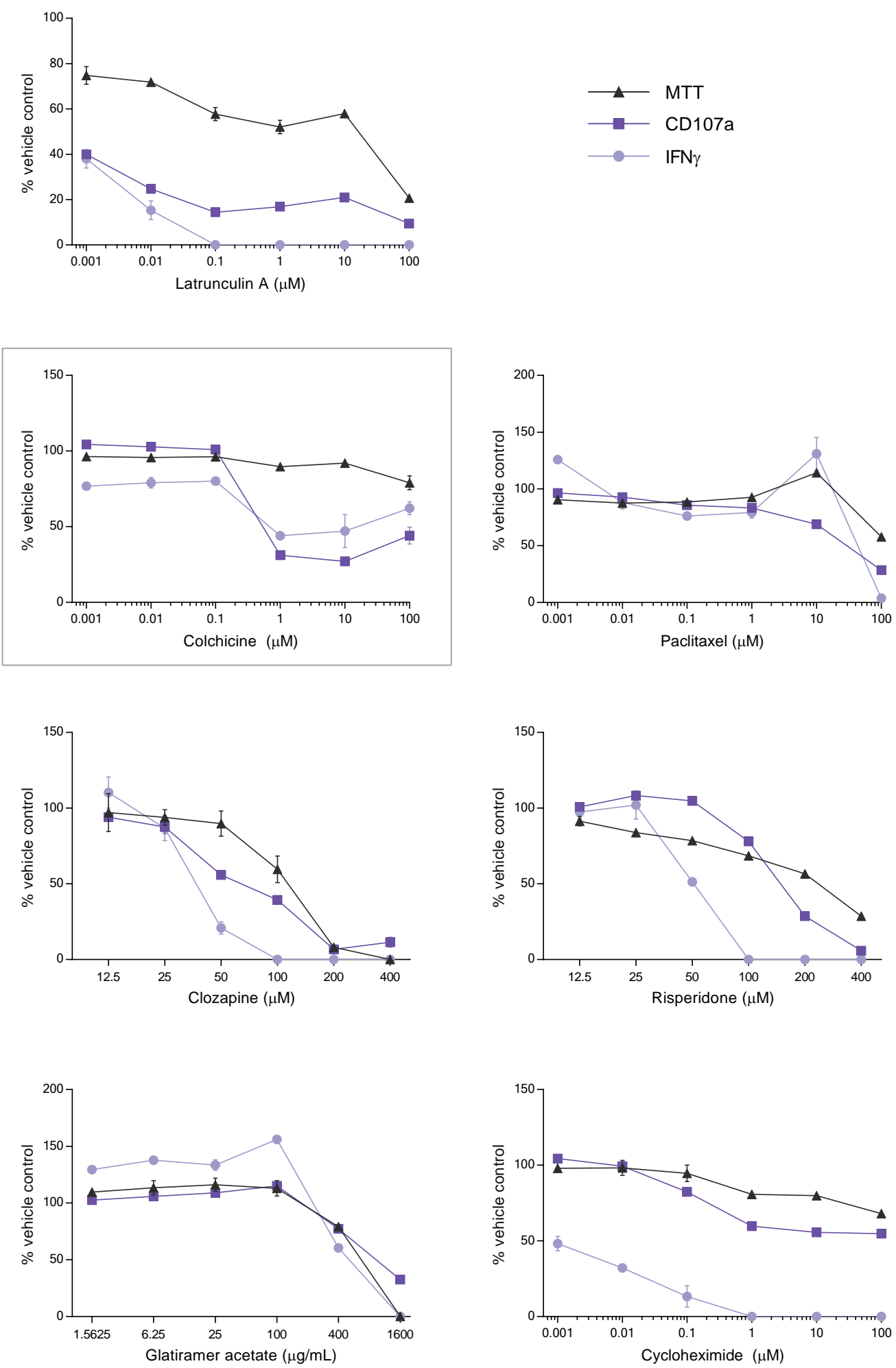

Figure 4.7. The effects of screen-building set compounds on degranulation by CTL

140,000 eCTLs were seeded into wells of a 96 well plate, and pre-treated with indicated treatments, or vehicle equivalents, for $4 \mathrm{~h} .25 \mathrm{nM}$ SIINFEKL and $1 \mu \mathrm{g} / \mathrm{mL}$ CD107a were then added to wells to initiate and detect degranulation, alongside further treatment to maintain concentration. After two hours, IFN $\gamma$ was measured from supernatants by ELISA, and cells were subjected to flow cytometry to assess degranulatory activity. Cells were pre-treated in parallel, and subjected to MTT assay to determine cell viability before degranulation was initiated (black). Data are means and SEM from one experiment conducted in duplicate; Colchicine data presented here are the same as presented in Figure 3.19. 


\subsubsection{Screen-building set compounds: effects on B cell activity}

This assay detects changes to B cell activity induced by test compounds. Cycloheximide was used as a positive control as it inhibited IgM and IL-10 levels at $0.001-0.01 \mu \mathrm{M}$; at these concentrations, IL-6 remained unaltered by cycloheximide (Figure 4.8, grey box). Above $0.01 \mu \mathrm{M}$ cycloheximide B cell viability diminished, reflected by decreased cytokine and antibody production.

LatA treatment strongly reduced viability with the lowest concentration tested, reducing cell viability to $\sim 75 \%$ of vehicle-treated cells. At this concentration of LatA, IgM levels were approximately half of vehicle-treated cells. In contrast, IL-6 and IL-10 levels remained stable despite the changes in cell viability, until the highest concentration was reached.

Colchicine also affected cell viability in this assay, and whilst IL-10 and IgM levels correlated with viability, IL-6 levels rose dramatically. At the lowest concentration (i.e. $0.001 \mu \mathrm{M}$ colchicine), IgM, IL-10 and IL-6 production was not affected. Paclitaxel had only a modest effect on viability, reducing to $80 \%$ of vehicle control across all concentrations of drug. IgM, and IL-10 levels were reduced. In contrast, as observed with colchicine, IL-6 levels were elevated and sustained at concentrations above $0.01 \mu \mathrm{M}$ paclitaxel.

Clozapine's main affect appeared to be on cell viability, where all changes reflect the decreasing metabolic activity of treated cells. Risperidone also affected cell viability, though at $25 \mu \mathrm{M}$ where viability was $\sim 77 \%$, there was a large increase in IL-10 - an effect not seen with the other atypical antipsychotic. Glatiramer acetate only modestly reduced IgM production and had little effect on IL-10 or viability at concentrations of $400 \mu \mathrm{g} / \mathrm{mL}$ or below. The major effect of glatiramer acetate was the large increase in IL-6 production, which rose to levels double those of vehicle-treated cells at $400 \mu \mathrm{g} / \mathrm{mL}$.

Again, the different compounds induced different changes to B cell activities. What is most interesting is the lack of difference in viability between unstimulated and stimulated B cells (see appendix 9.4.3, pg 208). It suggests that the targets of these compounds are similarly active in both $\mathrm{B}$ cell states. 

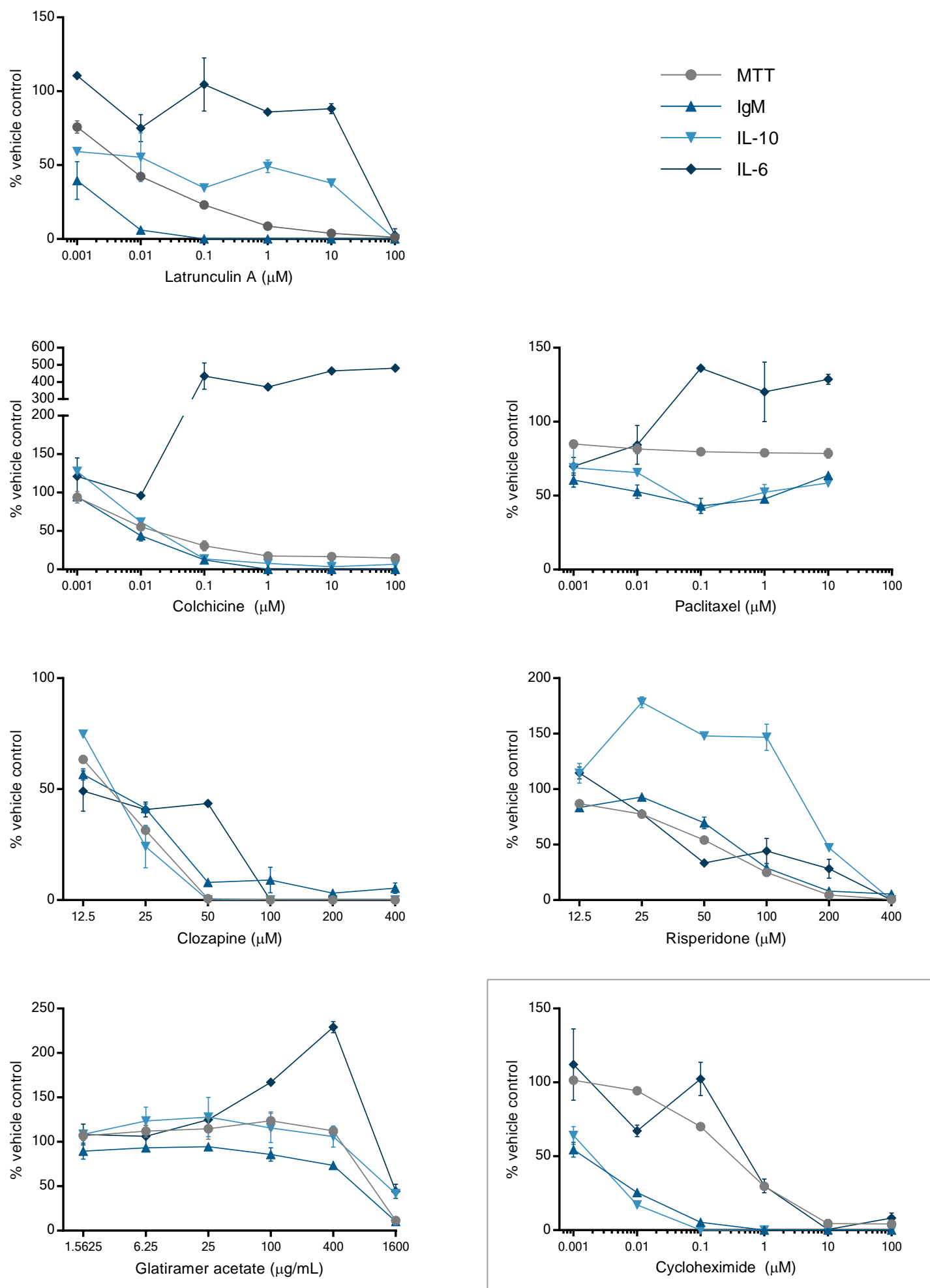

Figure 4.8. The effects of screen-building set compounds on $B$ cell activity

$1 \times 10^{6}$ splenocytes were seeded into wells of a 96-well plate and stimulated with $0.1 \mathrm{ug} / \mathrm{mL}$ LPS alongside indicated treatments, or vehicle equivalents. After $48 \mathrm{~h}$, cytokines and IgM concentrations were analysed by ELISA from supernatants, and cells were subjected to MTT assay to determine cell viability. Data are means and SEM from one experiment conducted in duplicate. Cycloheximide data presented here are the same as presented in Figure 3.25. 


\subsubsection{Activity summary of the compounds in the screen-building set}

To summarise the activity of this chapter's compounds across the assays, it was necessary to simplify the data set. This was done by classifying changes to activity into one of five categories. Changes to cell activity that were induced by compounds to be between 75 $125 \%$ of vehicle control activity were not considered to represent a robust change in cell activity; these are presented in white in the activity profile summary (Figure 4.9). Decreases (grey) between $20-75 \%$ of vehicle control were considered moderate, and decreases below 20\%, including the complete absence of output signal, were considered large decreases in activity. Similarly, increases (yellow) to outputs between 125 - 180\% control were moderate while increases above $180 \%$ were considered large. Black indicates no data were collected. These categories were based upon an assessment of the whole data set, whereby one third of the data fell into the basic categories: no change, moderate change, and greater changes.

Figure 4.9. Activity profiles of the compounds in the screen-building compound set (opposite) Data from the assays are summarised as activity profiles. Increases to cell function induced by compounds are represented in yellow, whilst decreases are shown in grey. The intensity of colour is indicative of the magnitude of change. White shows no change was observed, whilst black indicates no data were collected. 

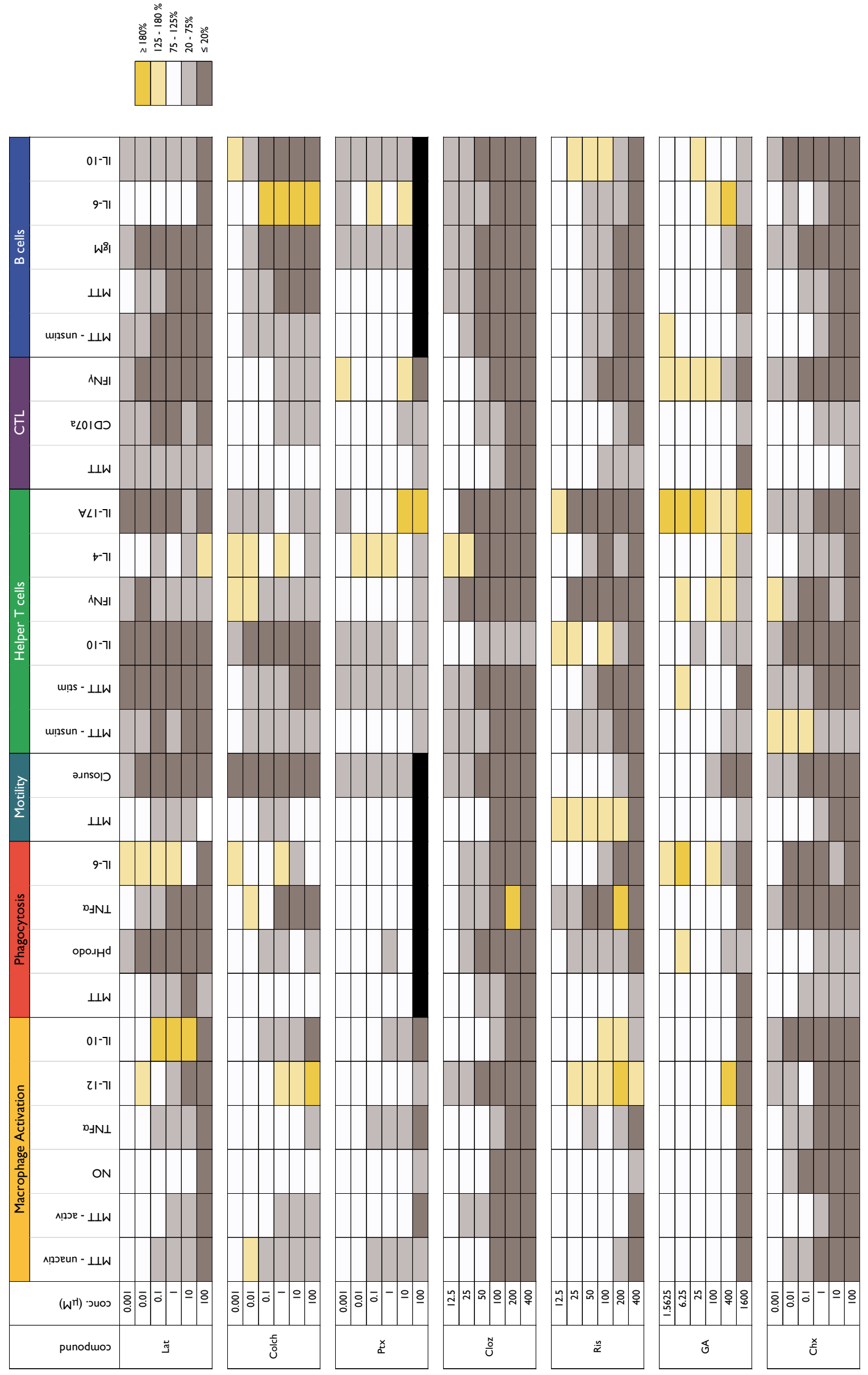
The activity profiles were used to summarise the activities of the compounds tested (below). The data generated in this chapter are also used to develop the screening strategy which is discussed later in this chapter.

Latrunculin A: Suppresses lymphocyte activity through cytotoxicity. Suppresses phagocytosis and motility through actin microfilament disruption. Alters macrophage activation type.

Colchicine: Anti-proliferative, as seen with greater toxicity against proliferating unactivated RAW 264.7 macrophages. Inhibits motility and phagocytosis, and alters cytokine production of activated macrophages. Lymphocyte cytokine production also affected, as well as $\mathrm{T}$ cell activation and degranulation. Effects are explained through microtubule destabilisation as cytoskeletal dynamism is required for these processes[152,153]. Generally, at non-toxic doses, many immune activities were suppressed.

Cycloheximide: Suppresses cytokine production in all cells through inhibition of protein synthesis. More toxic to active or proliferating cells, likely due to their need to produce new proteins to proliferate and/or maintain relatively high metabolic activity. Cycloheximide also inhibits cell motility through an unknown process.

Paclitaxel: Reduces viability in activated T cells and unactivated RAW 264.7 cells, likely through anti-proliferative microtubule stabilisation. The same mechanism inhibits motility through cytoskeletal disruption. The production of cytokines from macrophages, and cytokines and IgM from B cells is also generally suppressed. 
Glatiramer acetate: Few effects on macrophages, but concentration-dependent effects on phagocytosis and concurrent IL-6 production. Also decreases motility. Has proinflammatory effects through T cells: IL-17A and IFN $\gamma$ increased and IL-10 suppressed, though elevated IL-4 seen with high concentrations. B cells were also altered as IgM antibody production was suppressed, whilst IL-6 and IL-10 slightly increased. These cytokine changes suggest an overall pro-inflammatory effect with glatiramer acetate treatment. This differs from what is seen in multiple sclerosis patients, which may be an effect of the in vitro setting, or the lack of active myelin-specific T cells.

Clozapine: Suppresses immune cell activity. Lymphocytes are susceptible to drug, though this may be a problem with the concentration range used being too high. Many macrophage activities are suppressed, including cytokine production, motility, phagocytosis and concurrent cytokine production are reduced with treatment.

Risperidone: Main effects are on cytokine production on both lymphocytes and macrophages, with a general shift towards anti-inflammatory state in $\mathrm{T}$ and $\mathrm{B}$ cells, highlighted by decreased pro-inflammatory TNF $\alpha$ (macrophages) and IFN $\gamma$ production (T cells), and increased production of anti-inflammatory IL-10 (macrophages, T and B cells). Phagocytosis and cell motility were suppressed in macrophages. 


\subsection{Discussion}

In this chapter a data set was generated by testing several compounds through the assays optimised in the previous chapter. This work offered the chance to re-evaluate the assays and determine whether any of the proposed work was not informative and therefore not worth undertaking. Running an increased number of compounds through the assays also gave invaluable insight into the limitations of assay throughput. The generated data also gave insight into how the data from these assays relate to one another and were used to build the screening strategy. This section first discusses aspects of the assays which is followed by the development of the screening strategy.

\subsubsection{Observations from assays}

Overall, assays performed well. Their designs suited medium-throughput drug discovery and were suitable for detecting change induced by test compounds. When the data were collated by drug, as seen in the activity profiles, the observed changes reflected what is known and expected of the compounds' activities. Some produced data are novel, so not every effect of each compound could be verified.

Each assay, and almost every assay output, was altered by at least one compound with the exception of NO production by activated macrophages. Although the Griess reaction is a quick and cheap assay to run to assess NO, none of the compounds used in this chapter altered this cellular process. This may be due to the design of the assay, perhaps NO production cannot be altered with these activation conditions, or it is because the activities of the compounds tested do not alter this macrophage activity. Because this question remains, the utility of this measurement needs further investigation.

In the same assay it was noticed that changes to RAW 264.7 cell cytokines by test compounds did not resemble changes to BMDM cytokines. It was a characteristic of the RAW 264.7 cells used in this project, and not of risperidone in particular. It was decided that the priority was to use RAW 264.7 cells for this assay. These cells are more suitable for drug discovery due to their relatively simple culturing requirements, and adequately detect a compound's ability to alter macrophage cytokine production. If changes are seen in RAW 264.7 macrophage activation, compounds can be tested with activated BMDM to 
assess changes to IL-10 and IL-12 production. This approach also reduces the demand of animals required to complete the set of assays.

Using zombie dyes to assess cell viability during flow cytometry experiments was also investigated as a means to reduce the resources required during the phagocytosis and degranulation assays. When comparing cell viability across seven compound treatments, it appeared that data generated from the zombie dye method were less informative than data from MTT assays. The compromise of using MTT to assess viability during these assays is the need to plate and treat additional cells so that one set is available for the MTT assay. This almost doubles the resources required for the phagocytosis and degranulation assays. However, as discussed later in this chapter, cell viability during these two experiments can be inferred from other assays, thus eliminating the need for either dye exclusion or MTTbased viability assessment during these assays. Thus, there is no need for the duplicate plate.

In this chapter, immunomodulation was seen to be caused by a wide range of compounds. For many of the compounds, the changes were expected. What was interesting was the variability of changes induced by this small set of compounds. The different effects of microtubule stabilisation and destabilisation was not expected and, though it may be due to paclitaxel and colchicine specifically, are interesting. The two atypical antipsychotics also demonstrated very different effects: Clozapine was broadly suppressive, whilst Risperidone tended to promote anti-inflammatory IL-10 production. As the assays measure changes in activity across several immune processes, the assays provided a breadth of information about tested compounds which can identify these differences between compounds. Fewer assay outputs, e.g. screening only for toxicity, would not identify these differences.

Whilst beneficial for understanding compound activity, the assays generate a somewhat large amount of data, so activity profiles were made to simplify the interpretation of a compound's effects on the different immune cell processes. The intention behind these profiles was to highlight the activity of the compound, and at a glance identify which activities of a compound should be further investigated. Because the strategy measures many different cell outputs across the six assays, the activity profiles can be looked at together to generate a hypothesis about the activity of the compound. For example, reductions in IFN $\gamma$, IL-12 and TNF $\alpha$ suggests those compounds should be further 
investigated for anti-inflammatory activity. Alternatively, significant changes to RAW 264.7 proliferation, cell motility, degranulation and phagocytosis can suggest a compound alters the cytoskeleton. To generate the activity profiles, changes to assay outputs were classified into one of five categories, however the thresholds for each of these categories were difficult to define. Categories were based on magnitude of changes, but if the full range of change has not been achieved in this data set, the thresholds may need to change. Despite this, the activity profiles offer an informative snapshot of the immunomodulatory activity of test compounds. It not only summarises a compound's activity, but also enables comparisons to be made between compounds.

\subsubsection{Considerations for the screening strategy build}

The hypothesis that assay outputs may be linked arose from the shared mechanisms that cell processes use. Phagocytosis and cell motility both rely on cytoskeletal rearrangements, hence it was hypothesised that decreases to one of these functions would be reflected by changes in the other, and that these two functions would always correspond. If two cell processes corresponded, it would eliminate the need to assess compounds against both in the early stage of compound screening. This was shown not to be the case. Using the example, cycloheximide inhibited motility whilst leaving phagocytosis intact. The variety of compound-induced changes seen in this chapter showed that no immune processes were linked in terms of their modulation, and that data from each assay would be required to characterise a compound's activity; changes to any one process cannot be assumed from another. The hypothesis failed to acknowledge the wide array of mechanisms involved in cellular processes (e.g. that protein synthesis is also important for motility, as seen with cycloheximide), and that the different mechanisms through which compounds act can cause different changes to immune cell activity (e.g. MT stabilisation vs destabilisation), even if the same general target is shared (i.e. microtubules).

However, cell viability is one parameter that can be safely inferred between assays. The predominant interest is in immunomodulatory activity when compounds are non-toxic to cells (75\% viability of vehicle controls, or higher) so that any observed changes to cell activity are not due to changes in cell viability. Therefore, only testing non-toxic concentrations of compounds is more informative of compound activity, and only testing 
these concentrations through the assays would ensure time and resources were used optimally.

The assay with highest sensitivity to cytotoxicity can be used to identify the non-toxic concentrations of each compound that should be used for the remaining assays. This needs to be done for each cell population as the concentrations that are non-toxic to macrophages does not necessarily reflect non-toxic concentrations for splenocytes. Non-toxic concentrations of compounds can be determined by MTT data from activated macrophages during the macrophage activation assay, and from unstimulated cells during the helper $\mathrm{T}$ cell activation assay. These assays are both sensitive to cytotoxicity, but non-toxic concentrations of compounds in these assays are also non-toxic in other assays of the same cell type. For example, concentrations of compounds that were non-toxic to activated macrophages were non-toxic during the phagocytosis and motility assays. Similarly, concentrations that were non-toxic to unstimulated splenocytes were non-toxic to activated Th cells, B cells and CTLs. By identifying appropriate compound concentrations early, and using this to guide the concentrations to use through remaining assays, the repeated testing of toxic concentrations of compounds can be avoided, therefore the subsequent generation of less informative data is minimised. This also reduces the amount of compound required to complete the assays as the higher, toxic, concentrations are identified and avoided. This is especially important when novel compounds are being investigated for bioactivity before large scale production of the compound has commenced.

Identifying the non-toxic concentrations of compound means that additional viability assessments do not need to be made during the phagocytosis and degranulation assays. However, this may present some additional problems: the phagocytosis and degranulation assays are tolerant of high concentrations of compounds that would normally kill cells in other assays. This may also mean that the non-toxic concentrations - determined from more sensitive assays - are ineffective in these assays. For phagocytosis, the non-toxic concentrations determined by the macrophage activation assay showed activity against phagocytosis where expected and therefore was not identified as a problem. However, this was not the case for the degranulation assay. The non-toxic concentration of Colchicine (0.001 $\mu \mathrm{M})$ judged from $\mathrm{T}$ cell activation data does not inhibit degranulation. This suggests, perhaps, that colchicine would interfere with T cell activation and therefore naïve CTLs would normally not become effector CTLs with colchicine treatment. Alternatively, 
it highlights that concentrations may not translate between the helper T cell and CTL based assays. More data may show if the degranulation assay remains informative with the proposed changes to concentration selection, and identify any changes that need to be made to the decision made in this discussion.

Additionally, viability would still be assessed during the motility assay despite non-toxic concentrations being used in the assay. This assay has a significantly longer duration, and uniquely uses BMDM. The viability of stimulated B cells would also be assessed. This information may provide insight into cell-specific targets of compounds. For example, compounds at concentrations that are non-toxic for resting splenocytes may decrease B cell viability, suggesting the compound alters B cell activation. At the same time, the cells in these assays are not required for any other assessment and are available for the MTT assay.

Lastly, wide titrations of test compounds should be tested during the macrophage activation and helper T cell assays to identify the concentrations of compounds that should be used. This approach would have identified suitable concentrations of Clozapine to use on splenocyte-based assays. In this chapter, Clozapine concentrations were based on literature, and it was not appreciated at the time that this concentration range was specific to macrophage assays. 


\subsubsection{Designing the screening strategy}

Taking the information learned during this series of experiments led to the design of the screening strategy which is described here.

The first stage of the screening strategy identifies the concentrations of compounds that should be tested throughout the screen. This is done twice: once for macrophage work, and again for splenocyte work. To do this, wide titrations of compounds are tested through both the macrophage activation and Helper $\mathrm{T}$ cell activation assays. MTT data from activated macrophages and unstimulated $\mathrm{T}$ cells identify which concentrations are nontoxic ( $\geq 75 \%$ viability) which can then be used throughout the remaining assays. Supernatants from these experiments are analysed later, after all assays have been completed.

Acknowledging that all assays need to be run, the rest of the strategy design is led by looking at the resources required for each assay. As RAW 264.7 cells are required for the first step of screening, it was sensible to conduct the phagocytosis assay soon after. This is the only other assay that requires this cell line, therefore once the phagocytosis assay is completed RAW 264.7 cells no longer need to be in culture.

The remaining assays use primary cells. To reduce animal use, (i.e. make the most of a mouse sacrifice), the same set of mice can provide cells for both the B cell activation assay (spleen) and for the assessment of cell motility (bone marrow). For this reason, these two assays are next in the workflow and the B cell activity assay can be completed whilst bone marrow harvests were differentiated into macrophages for the motility assay. The degranulation assay remains, which uses spleens from OT-1 mice. This assay was placed last in the strategy as it is convenient to process and prime the spleens during the later stages of the bone marrow culture. However, this assay can happen at any time after concentration identification as the OT-1 mice are only used for this assay.

Most of these assays have outputs where cytokine levels are measured from cell supernatants. It was more time effective to run several ELISAs together, and for this reason time is better spent collecting supernatants from each experiment and analysing their contents at the same time at the end of all experiments. Arranged in this way, the workflow 
is estimated to take four weeks to complete. The ideas discussed here make up the screening strategy, and are summarised in the following schematic (Figure 4.10).

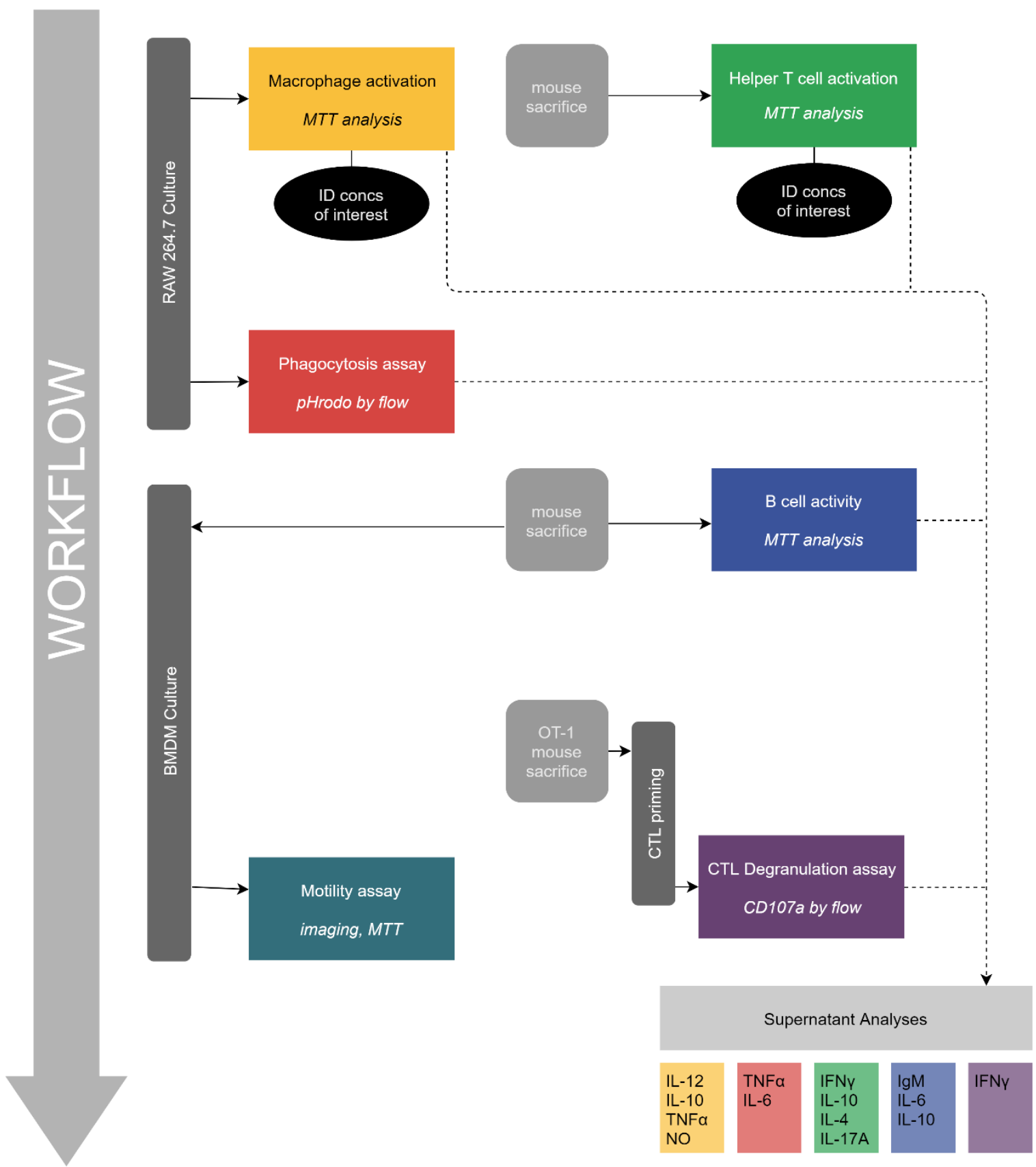

Figure 4.10. A schematic of the proposed screening strategy.

This workflow describes, from top to bottom, the order by which compounds should be tested throughout the assays. This not only assesses immunomodulatory activity, but also identifies concentrations of compounds to use in the assays. Assays are arranged to reduce resource requirements and streamline certain analytical processes (i.e. ELISAs). 


\subsection{Summary}

In this chapter, the testing of positive controls (i.e. the screen-building compound set) through all the assays offered insight into assay sensitivity and informativeness. The assays were deemed appropriate for detecting immunomodulation caused by a variety of compounds. The data were also used to design a screening strategy. The benefit of arranging these assays into a streamlined workflow is the increased efficiency of completing the six assays: this screening strategy reduces costs and reduces the amount of test compounds required to complete the experiments. The screening strategy was built retrospectively from a data set generated from testing compounds through assays separately, so the proposed screening strategy requires testing to ensure the generated data are indeed informative and that the data are valid. This is explored in the next chapter. 


\section{Chapter 5: \\ Evaluating the performance of the screening strategy}




\subsection{Introduction}

A screening strategy was designed in the previous chapter. Its ability to identify the immunomodulatory activities of compounds will be evaluated in this chapter. To do this, compounds with known bioactivities will be de-identified and run through the proposed strategy. These results, and information from the literature, will be used to identify the compounds. This blinded approach will enable the system to be tested in an unbiased manner and, if successful, will demonstrate the validity of data generated using the screening strategy.

Also, working with coded compounds should replicate novel compound testing, where testing concentrations and activities are unknown, and a larger number of compounds would be tested together. This approach will highlight any areas of the screening strategy that need further optimisation or development.

The compounds chosen to test the screening strategy have a wide range of activities which are expected to produce a wide range of activity profiles. This should also allow for an adequate evaluation of the utility of the screen assays. This includes NO production by activated macrophages and the CTL degranulation assay: two screening components that were previously identified as potentially being less informative.

\subsection{Aims}

The aim of this chapter is to evaluate the proposed workflow via three objectives:

1. Run coded compounds through the screening strategy

2. Use literature to identify coded compounds based on generated activity profiles

3. Evaluate the performance of the strategy design to characterize compounds; that findings reflect independent observations. 


\subsection{Results}

Twelve compounds were assembled to test the screening strategy (Table 5.1). These compounds have known targets, including inhibitors of kinases important for immune cell activation (e.g. ibrutinib, rapamycin) and compounds that have more general cellular targets (e.g. doxorubicin, brefeldin A). Many compounds have well-documented effects on the immune system (e.g. gliotoxin, dopamine) while other compounds, such as chloramphenicol and BPS, have known mechanisms but have not been tested extensively for immunomodulatory activity. Ixabepilone was selected for its microtubule stabilising properties, akin to the previously screened paclitaxel, and aminoguanidine was included to determine if NO production could be altered. Compounds were made to stock solutions between $1-20 \mathrm{mM}$ in appropriate vehicles, and in an effort to blind the assessor, aliquots of the compound and its vehicle were coded using letters of the alphabet. As done previously, assay outputs for each compound were compared to their vehicle control and reported as a percentage.

Table 5.1. Compounds making up the validation set.

\begin{tabular}{|l|l|}
\hline Compound & \multicolumn{1}{|l|}{ Target/Mechanism } \\
\hline $\begin{array}{l}\text { Aminoguanidine (AMG) } \\
\text { (bathophenanthroline disulfonate) }\end{array}$ & Iron chelator \\
\hline Brefeldin A & Protein synthesis inhibitor at ER \\
\hline Chloramphenicol & Antibiotic \\
\hline Dopamine.HCl & Dopamine receptor activation \\
\hline Doxorubicin & $\begin{array}{l}\text { DNA intercalator: } \\
\text { Topoisomerase 2 inhibition }\end{array}$ \\
\hline Gliotoxin & Cysteine-binding, protein interruption \\
\hline Ibrutinib & BTK inhibitor \\
\hline Ibuprofen & NSAID, COX inhibitor \\
\hline Ixabepilone & Microtubule stabiliser \\
\hline Rapamycin & mTOR inhibitor \\
\hline Sulpiride & Dopamine D2 and D3 antagonist \\
\hline
\end{tabular}




\subsubsection{A modification to the concentration selection stage of screening}

As previously mentioned, the macrophage and helper $\mathrm{T}$ cell activation assays were designated the first step in the screening strategy as they identify the non-toxic concentrations ("concentrations of interest”) of compounds to use throughout the remaining assays. Therefore, for this chapter, each compound was titrated in these two assays to generate cytotoxicity data. Half-log dilutions of compound, between $0.00001-1 \%(\mathrm{v} / \mathrm{v})$ solution, were set up. This range covered concentrations between $1 \mathrm{nM}-100 \mu \mathrm{M}$ as most stock solutions were made to $10 \mathrm{mM}$. A call was made that any compounds that were toxic at $1 \mathrm{nM}$ were considered as having cytotoxicity as their main effect. To give an example from the previous chapter, LatA is considered toxic to splenocyte populations.

The MTT data from these assays would be used to identify the concentrations of interest. From these, two to four of the highest log-fold concentrations of each compound would be tested to identify immunomodulation and to capture any concentration-dependent effects of compounds. At the same time, MTT data would be used to generate cytotoxicity doseresponse curves from which $\mathrm{IC}_{20}$ values would be calculated and these concentrations would also be used throughout the remaining assays. The $\mathrm{IC}_{20}$ values would provide another means to determine non-toxic compound concentrations that may be a clearer approach to identifying high, non-toxic concentrations.

After running compounds through the two initial assays, an unexpected situation arose. Some compounds did not alter cell metabolism over this wide concentration range: no dose response curve for cell viability (MTT) could be generated where this was previously identified as an appropriate method to identify non-toxic concentrations of compound and therefore narrow down the number of concentrations of each compound to test.

Additional analyses were needed to reduce the number of concentrations of each compound to test in the screening workflow when compounds appeared to be non-toxic across all tested concentrations. From the macrophage and splenocyte stimulation assays, two cytokines from each assay were measured to guide concentration selection. From activated macrophages, IL-10 and IL-12 were measured as these two outputs were shown to be the most sensitive to modulation by compounds in the previous compound set. For similar reasons, IFN $\gamma$ and IL-10 were selected from the helper $\mathrm{T}$ cell activation assay. These 
choices were supported by the results from the first compound set where changes to these four cytokines frequently accompanied changes to other immune cell processes, thus offering the best chance to narrow down the concentration range of compounds where effects are seen. MTT and ELISA data were used together to narrow down the concentrations of each compound that should be used in the remaining assays and this was done separately for each cell type.

To summarise the updated concentration selection step, MTT data from activated macrophages or unstimulated splenocytes were used to identify any toxic concentrations to avoid for assays using the same cell populations. Cytokine data at non-toxic concentrations of each compound were then used to identify which of these should be tested based upon changes to these parameters. In some cases, for macrophage assays, a stark difference in viability was seen between proliferating and non-proliferating macrophages. In these cases, this parameter also identified the concentration range of compound that should be tested. At times, neither MTT nor cytokine data did not narrow down the concentration range to test. If this was the case, three or four high concentrations of compound were selected for testing. To give examples of this decision-making process, the concentration selection thought process is explained with a few examples. 
In the first example, viability-guided concentration selection is appropriate for both macrophages and splenocytes: the concentrations of interest for compound $\mathrm{E}$ could be determined by MTT data as cytotoxicity is observed ( $\mathrm{IC}_{20}$ 0.0008\%). No major differences were observed between activity states for both the macrophages (Figure 5.1, A) and splenocytes (Figure 5.1, C). Additionally, the macrophage cytokine data are concordant with the MTT data and support selection of similar concentrations of compound E for further testing (Figure 5.1, B). Similarly, compound E had an $\mathrm{IC}_{20}$ of $0.00016 \%$ in the helper $\mathrm{T}$ cell assay (Figure 5.1, C) with similar inhibitory effects observed at that concentration on IL-10 production. Including $0.0001 \%$ and $0.00001 \%$ solutions, the effect of compound $\mathrm{E}$ on reducing IFN $\gamma$ production is also captured (Figure 5.1, D).

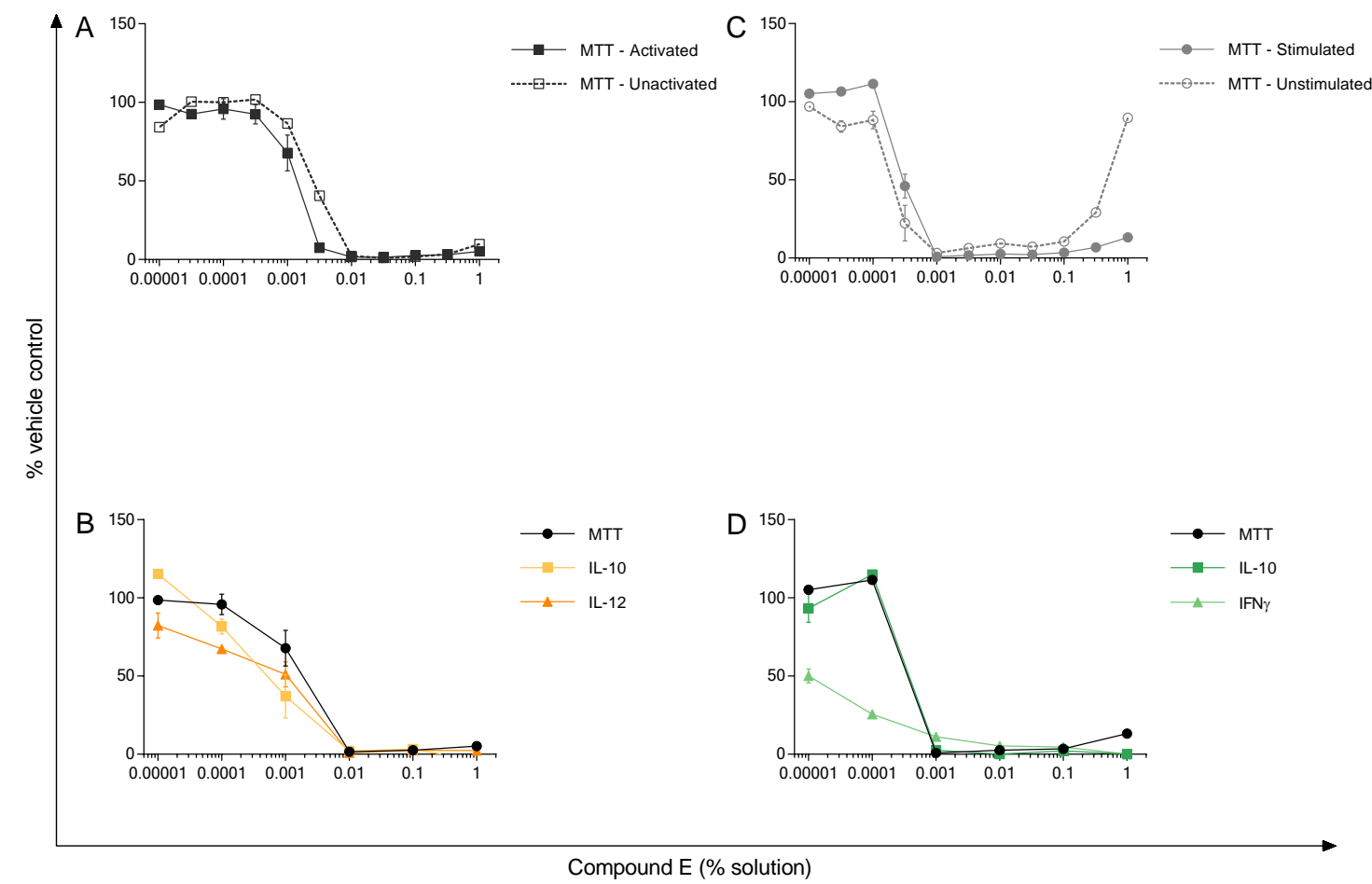

Figure 5.1. Testing concentrations for Compound $\mathrm{E}$ were determined by MTT data.

Compound E was run through the macrophage activation assay (A, B) and T cell activation assay (C, D). MTT (A, C) was measured on activated/stimulated cells (solid) and unactivated/unstimulated cells (dotted). IL-10 and IL-12 production was measured from activated macrophages (B) and IL-10 and IFN $\gamma$ was measured from activated T cells (D). Data are means and SEM from each experiment conducted once in duplicate. 
Compound $\mathrm{J}$ was non-toxic to both macrophages (Figure 5.2, A) and splenocytes (Figure 5.2, C) so cytokine data guided concentration selection for both cell populations. For macrophage-based assays, $0.01,0.1$ and $1 \%$ solutions were selected due to the effects seen on both cytokines (Figure 5.2, B). For splenocytes, 0.001, 0.01, 0.1 and 1\% solutions were selected. The highest three show non-toxicity, and the $0.001 \%$ solution increased IL-10 production in activated Th cells (Figure 5.2, D).
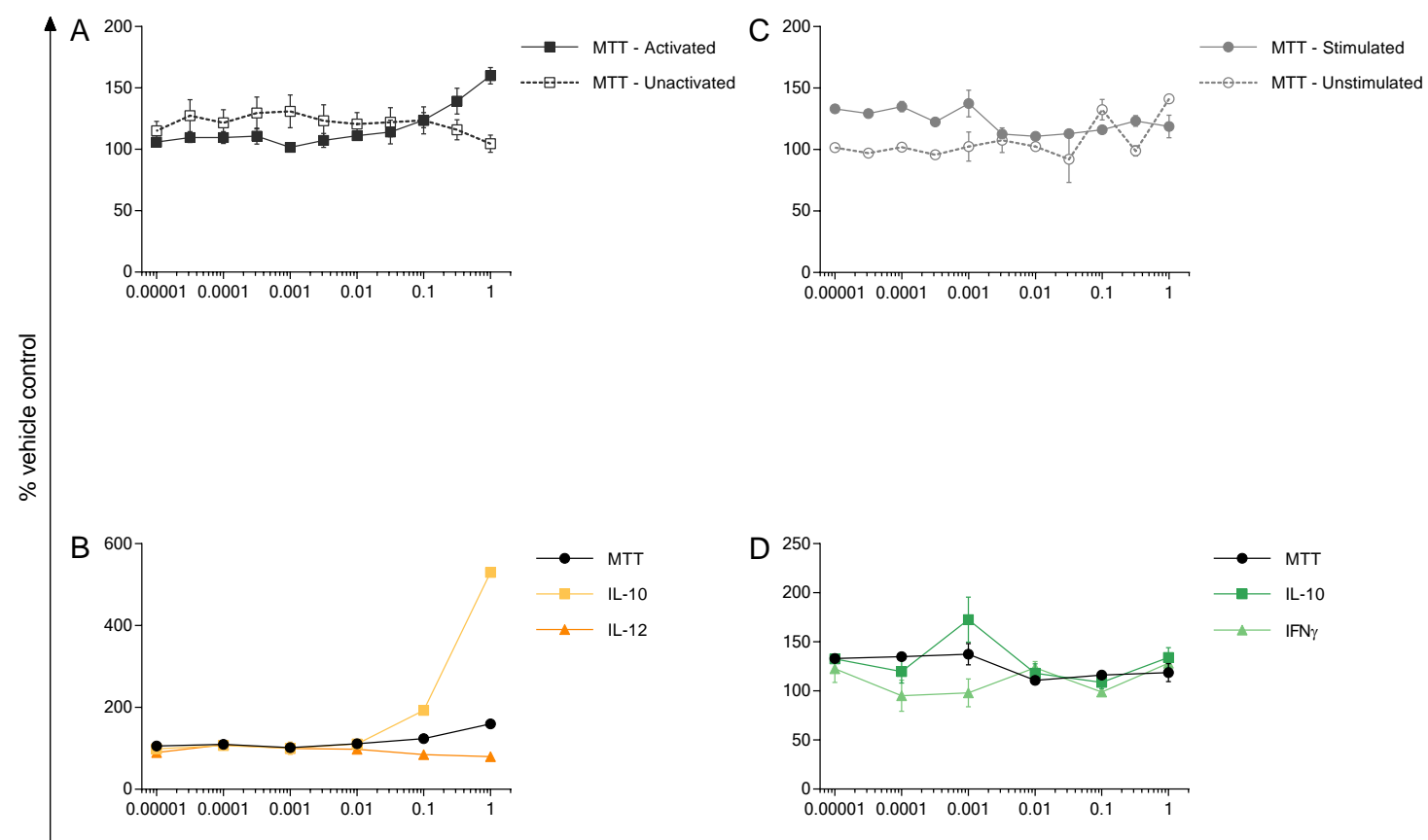

Compound $\mathrm{J}$ (\% solution)

Figure 5.2. Cytokine data guided compound $\mathbf{J}$ concentration selection.

Compound $\mathrm{J}$ was run through the macrophage activation assay (A, B) and $\mathrm{T}$ cell activation assay (C, D). MTT (A, C) was measured on stimulated cells (solid) and unstimulated cells (dotted). IL-10 and IL-12 production was measured from activated macrophages (B) and IL-10 and IFN $\gamma$ was measured from activated T cells (D). Data are means and SEM from each experiment conducted once in duplicate. 
Compound F had interesting cytotoxic effects on unstimulated macrophages, decreasing their viability relative to both vehicle control and activated cells; activated macrophages were not affected. For this reason, $0.001 \%$ and $0.0001 \%$ solutions were selected. The higher concentration caused different effects between the two cell states, whilst the lower caused no difference. These concentrations capture the apparent anti-proliferative effects of compound F, and may show any concentration-dependent effects of the compound. Higher concentrations could have been included, but this would not conserve compound. For splenocytes, three high concentrations $(0.01,0.1$ and $1 \%)$ of compound $F$ were selected as no effects to cell viability were seen. Similarly, no concentration-dependant changes were seen to cytokine production: the decreased IFN $\gamma$ is seen with all concentrations. Because $0.316 \%$ solution caused a spike in stimulated T cell MTT, it was included.

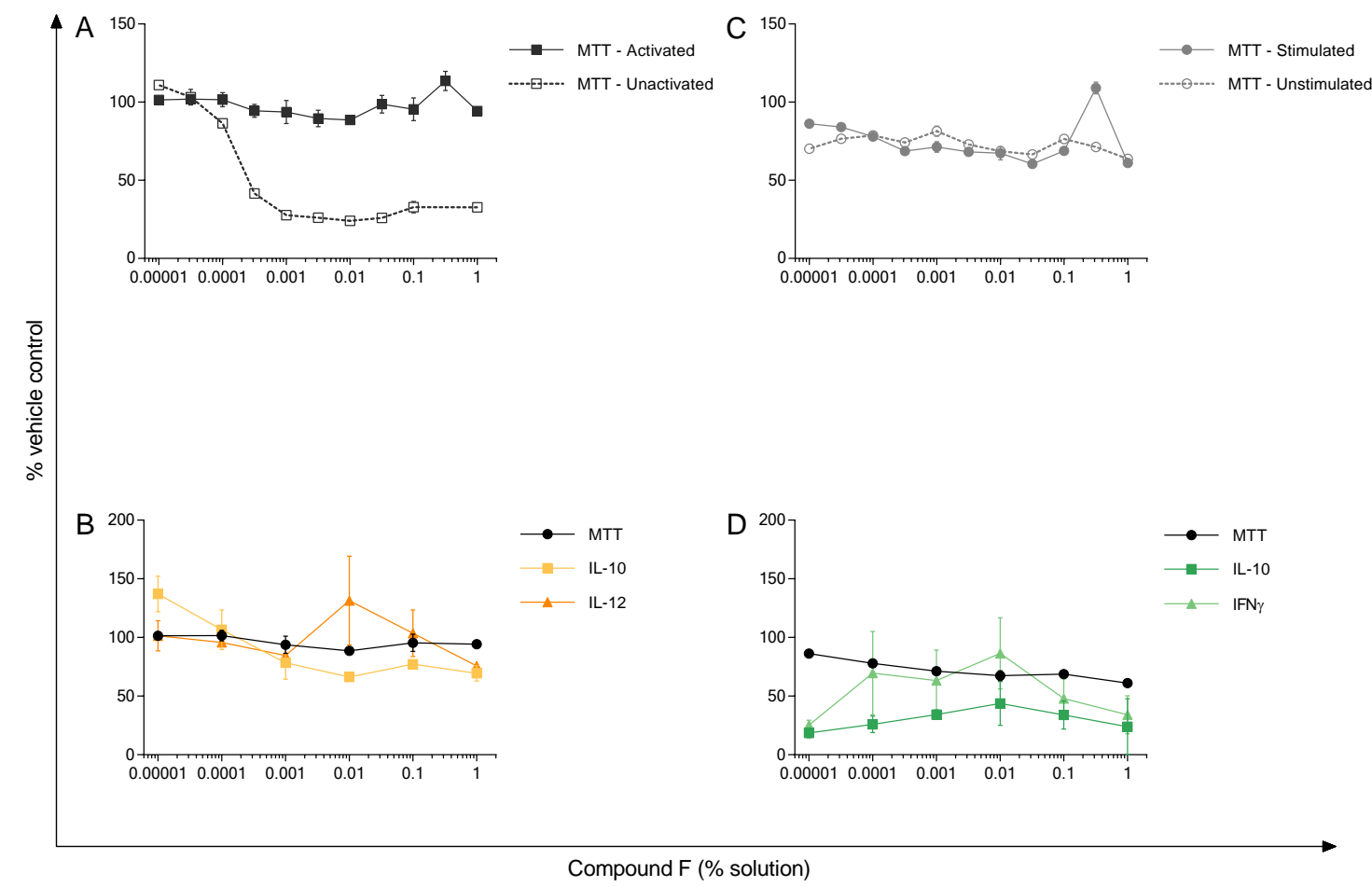

Figure 5.3. Compound $F$ concentrations were selected by a combination of methods, including using high concentrations when no concentration-dependent effect is seen during an assay.

Compound $\mathrm{F}$ was run through the macrophage activation assay (A, B) and T cell activation assay (C, D). MTT (A, C) was measured on stimulated cells (solid) and unstimulated cells (dotted). IL-10 and IL-12 production was measured from activated macrophages (B) and IL-10 and IFN $\gamma$ was measured from activated T cells (D). Data are means and SEM from each experiment conducted once in duplicate. 
In summary, this validation set of compounds revealed situations where viability data alone did not narrow down the relevant compound concentrations to use throughout the screening workflow. Instead, measuring select cytokines from each of the initial assays proved useful in identifying concentrations which were potentially bioactive. While it is possible to use the wide range of concentrations for all of the immune assays, narrowing down the range to those concentrations that are non-toxic but may have bioactivity reduces the experimental work required. This reduces costs by saving time and consumables, and also reduces the amount of compound required while maximising the relevant outputs gathered. More than one concentration of each compound was selected for further testing as interesting concentration-dependent effects were observed during this concentration selection step. When neither MTT nor cytokine production was altered by test compound, three high log-fold concentrations were selected for further testing. This updated workflow follows (Figure 5.4). 


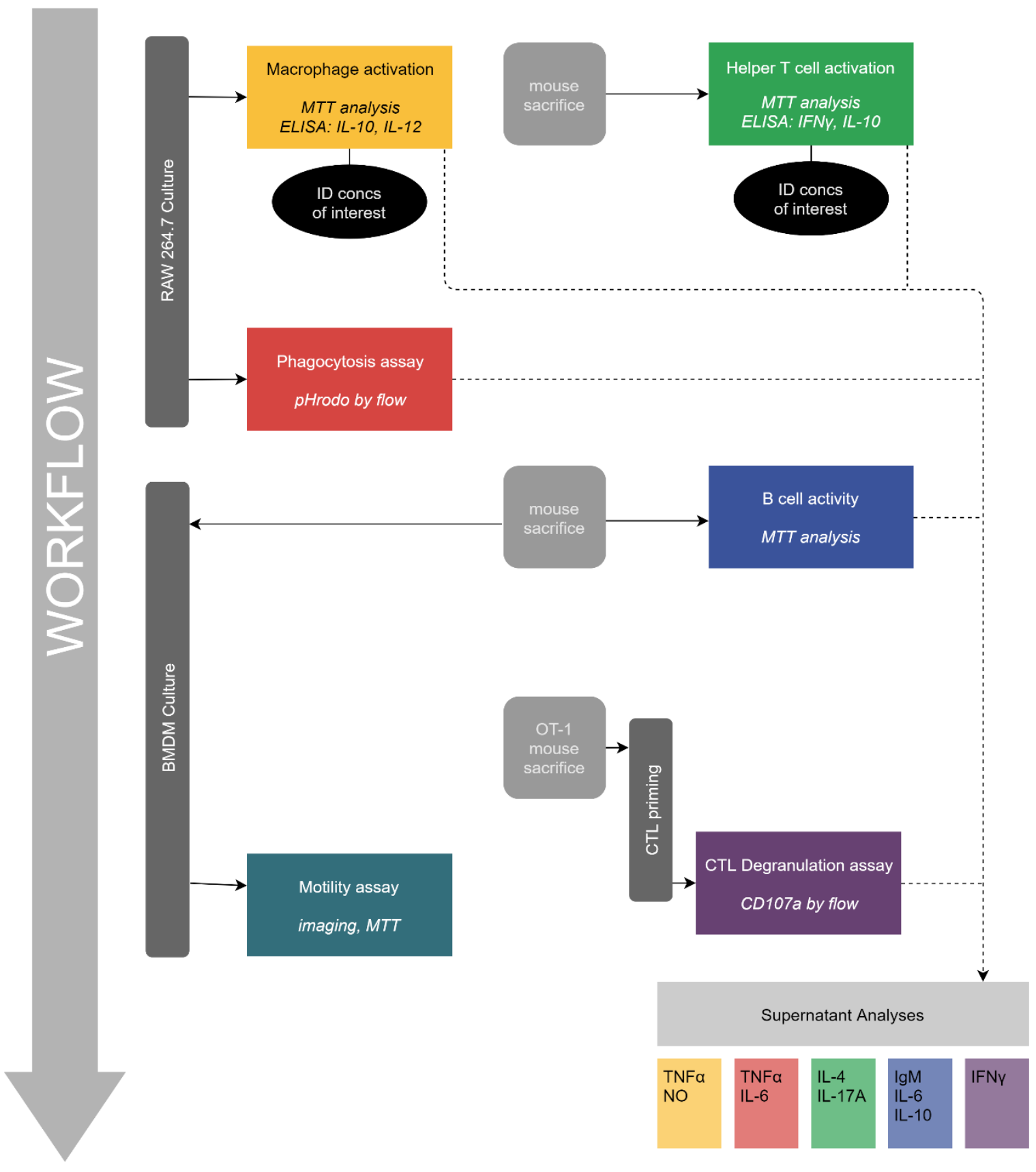

Figure 5.4. The updated screening workflow reflects the need to look at select cytokines during concentration selection.

The workflow schematic was updated to include the analysis of IL-10 and IL-12 during macrophage activation, and IL-10 and IFN $\gamma$ during helper T cell activation (compare to Figure 4.10 on page 124). These additional analyses aid in the identification of the concentrations of interest for each compound on each cell population. These are then used throughout the remaining assays of the same cell type. Macrophage assays are to the left of the schematic, whilst splenocyte assays are to the right. 


\subsubsection{The activity profiles of compounds in the validation set}

After narrowing down two to four concentrations of interest per compound, each was run though the remaining assays. For this validation set, the screening strategy was performed as depicted in Figure 5.4, and for brevity only an example of the screening data is shown below (Figure 5.5). The full data set is included in Appendix 9.5 (pg 209) where concentration identification and screening data are presented together by compound. The methods used to screen compounds follow the assay designs from Chapter 3, which are also described in the methods section. All experiments were conducted once, in duplicate.

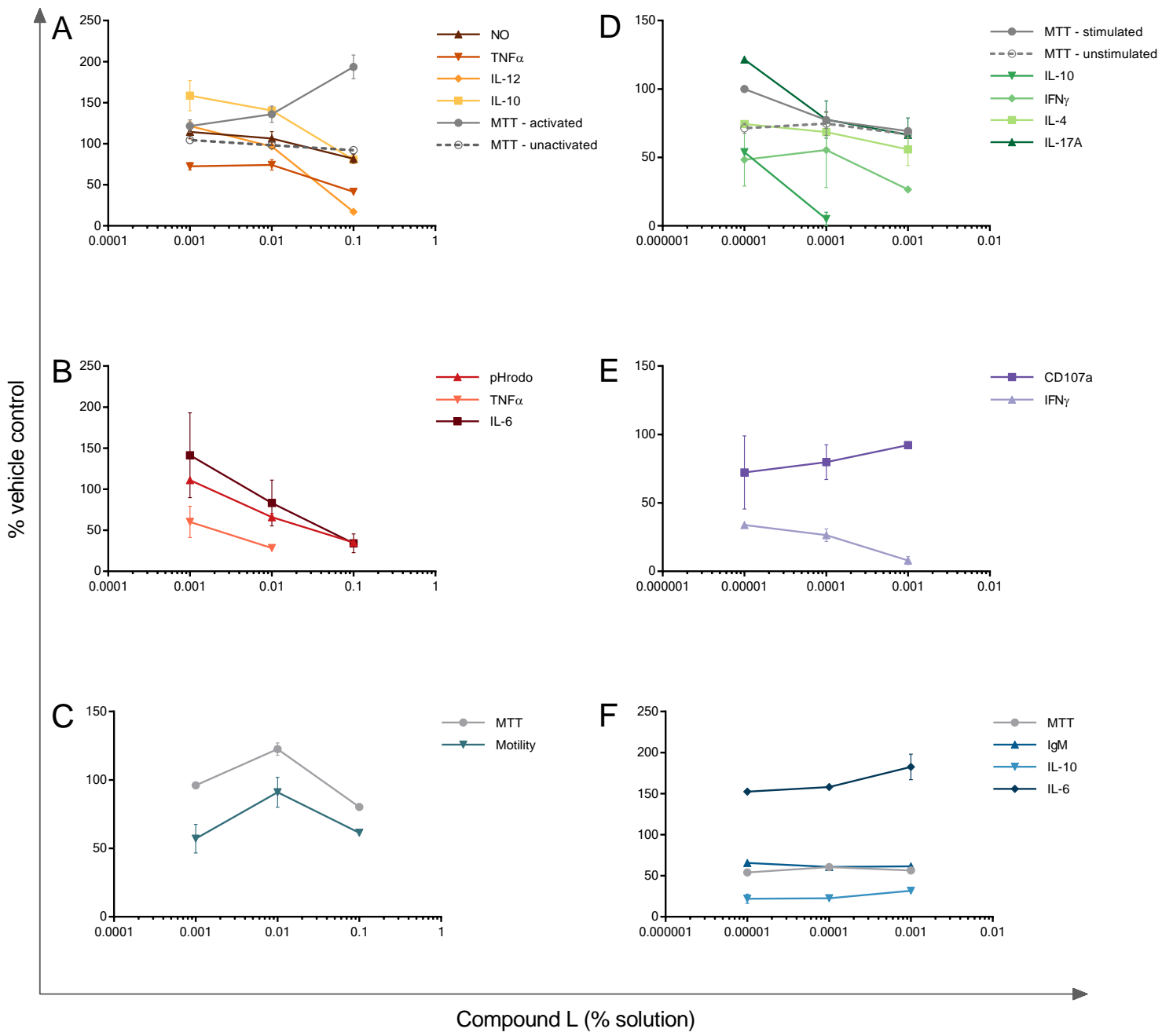

Figure 5.5. Screen outputs for Compound $L$ as an example of screening data.

Compound L, at indicated concentrations, were run through the six assays. Macrophage activation (A), phagocytosis (B) and motility (C) are macrophage-based assays. In the right column are the splenocyte-based assays: helper T cell activation (D), CTL degranulation (E) and B cell activity (F). Experiments were conducted as described in thesis methods (Chapter 2). Shown are compound-induced changes compared to vehicle controls (i.e. \% vehicle). Data are means and SEM from each experiment which was conducted once in duplicate. 
Two concentrations of positive controls were included in each experiment to ensure the assays operated as expected (Table 5.2). Risperidone replaced glatiramer acetate as the positive control for $\mathrm{T}$ cell activation modulation. This drug previously demonstrated its ability to alter $\mathrm{T}$ cell cytokine production and given its use in the macrophage activation assay, it was deemed more convenient to use Risperidone as a control for the two assays that run concurrently. The activity profile for the positive controls show that each assay worked as expected. That is, they modelled immunomodulation across six immune cell processes (Figure 5.6).

Activity profiles for test compounds were generated as in the previous chapter (section 4.3.10, pg 114). Briefly, percent-control data of treatments were assigned to one of five categories of change. Decreases in activity are represented in grey and increases in yellow where a darker colour indicates a larger change. No change remains white; black indicates no data were collected. The activity profiles for each compound follow. The data that were used to generate these profiles can be found in the supplementary material (Appendix 9.5, pg 209).

Table 5.2. Positive controls used in this chapter's experiments

\begin{tabular}{llc} 
Assay & Control compound & Concentrations used $(\mu \mathrm{M})$ \\
\hline Macrophage activation & Risperidone & $100,31.6$ \\
Phagocytosis & Latrunculin A & $0.01,0.001$ \\
Motility & Latrunculin A & $0.1,0.01$ \\
T cell activity & Risperidone & $31.6,10$ \\
B cell activity & Cycloheximide & $0.01,0.001$ \\
Degranulation & Colchicine & 10,1 \\
\hline
\end{tabular}
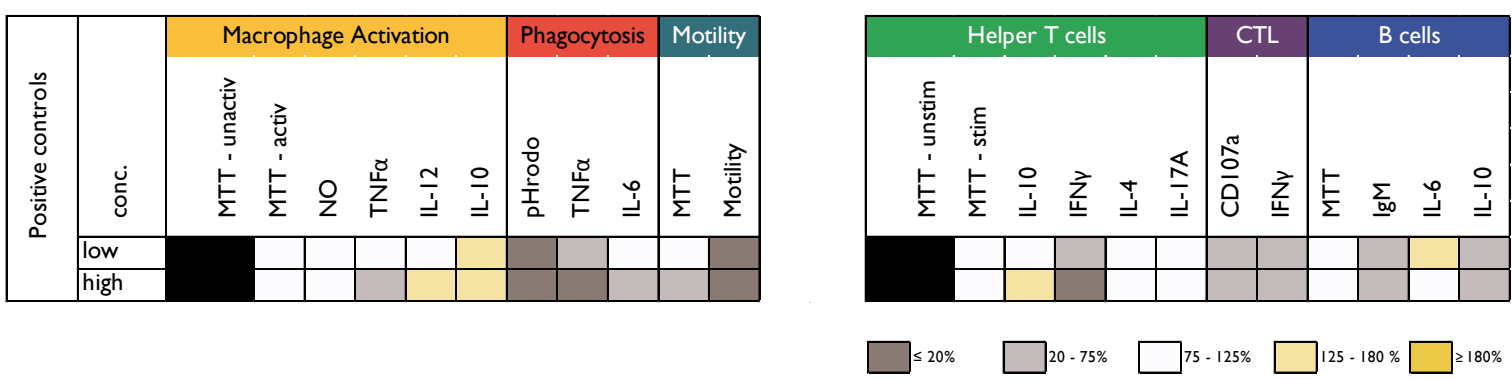

Figure 5.6. Positive controls demonstrate that each assay was conducted successfully.

Positive controls were run alongside compounds in this screening run. These controls differ for each assay (see Table 5.2, above) but are summarised together in this activity profile. This demonstrates that each assay was run correctly; that each modelled process was able to be modified. Increases are represented in yellow, and decreases in grey. White is no change; black indicates no data were collected. 

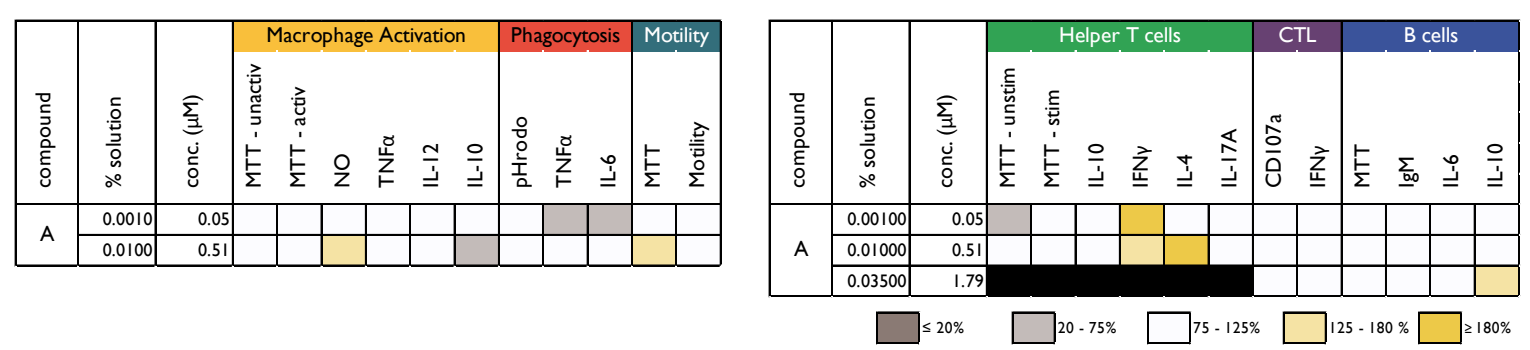

Figure 5.7. Activity profile for Compound A.

Categories are based on output levels compared to vehicle controls. Increases are represented in yellow, and decreases in grey. White is no change; black indicates no data were collected. Raw data can be found in appendix section 9.5.1.

Compound A has few effects on immune cells. For both macrophages and lymphocytes, only cytokine and NO production were affected; however, there was no clear bias towards a pro- or anti-inflammatory profile across the assays.
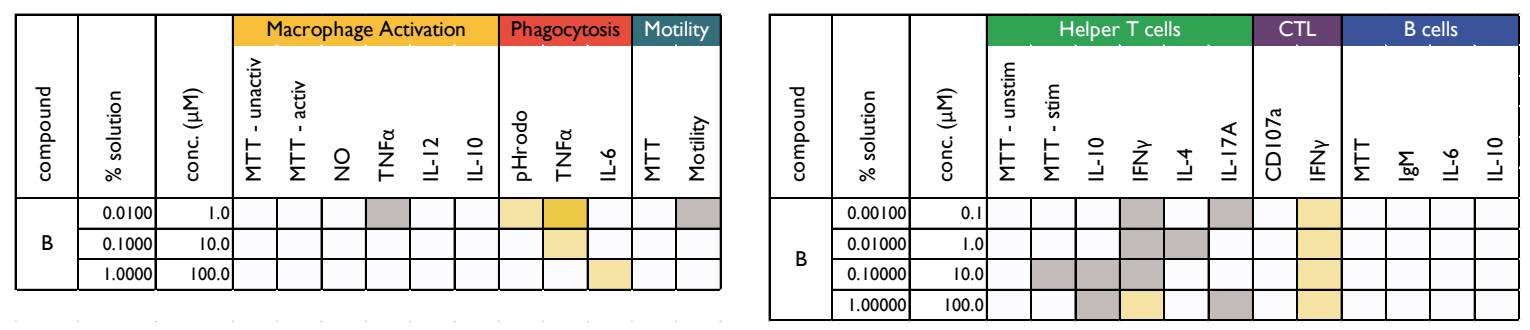

Figure 5.8. Activity profile for Compound B.

Categories are based on output levels compared to vehicle controls. Increases are represented in yellow, and decreases in grey. White is no change; black indicates no data were collected. Raw data can be found in appendix section 9.5.2.

Compound B enhanced phagocytosis at low concentrations, and this enhancement correlated with increased TNF $\alpha$ during this process. Activating T cells in the presence of compound B reduced cytokine production although IFN $\gamma$ by CTL was enhanced during the degranulation assay. B cells were unaffected by compound B treatment. 


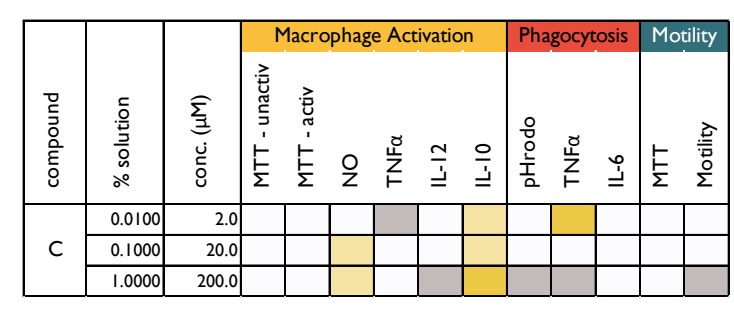

Figure 5.9. Activity profile for Compound C.

Categories are based on output levels compared to vehicle controls. Increases are represented in yellow, and decreases in grey. White is no change; black indicates no data were collected. Raw data can be found in appendix section 9.5 .3

Compound C demonstrated effects on macrophage activation, and at higher concentrations supressed motility, phagocytosis and concurrent TNF $\alpha$ production. At the highest concentration, general suppressive effects were seen on lymphocytes, which may be a result of the reduced viability, however lower concentrations saw enhanced IL-10 production by $\mathrm{T}$ and $\mathrm{B}$ cells. This suggests compound $\mathrm{C}$ has anti-inflammatory activity.
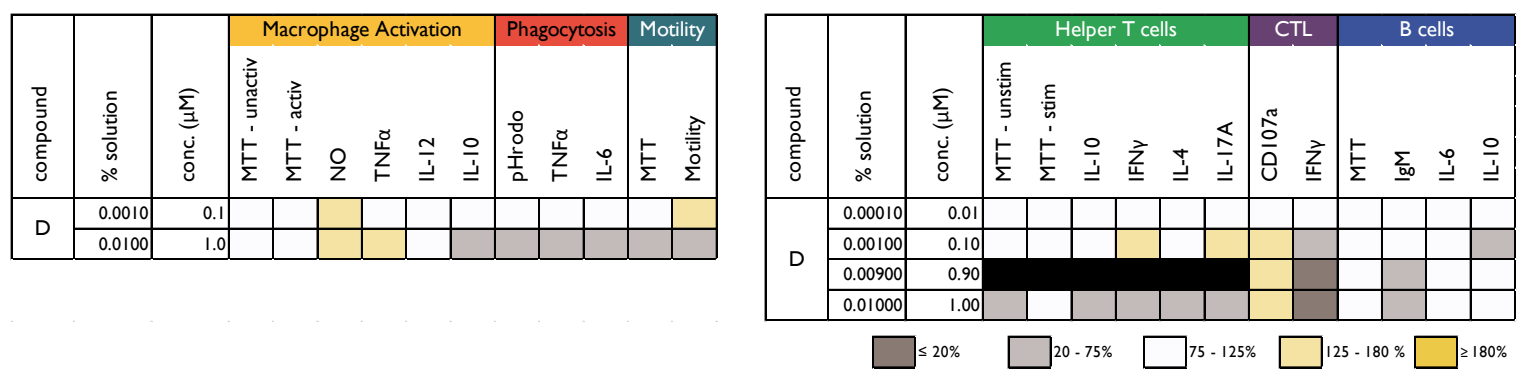

Figure 5.10. Activity profile for Compound $D$.

Categories are based on output levels compared to vehicle controls. Increases are represented in yellow, and decreases in grey. White is no change; black indicates no data were collected. Raw data can be found in appendix section 9.5.4.

Compound D moderately enhanced $\mathrm{NO}$ and $\mathrm{TNF} \alpha$ from activated macrophages, but inhibited IL-6, TNF $\alpha$, and phagocytosis during the shorter phagocytosis assay. Motility was enhanced with the lower concentration of compound D. Higher concentrations of compound $\mathrm{D}$ caused a decrease in the production of all $\mathrm{T}$ cell cytokines, and antibody production by B cells; viability was unchanged in these active cells but was decreased in unstimulated splenocytes. In contrast, lower concentrations slightly enhanced proinflammatory IL-17A and IFN $\gamma$. IFN- $\gamma$ from CTL was concentration-dependently decreased, and degranulation by CTL was enhanced. 


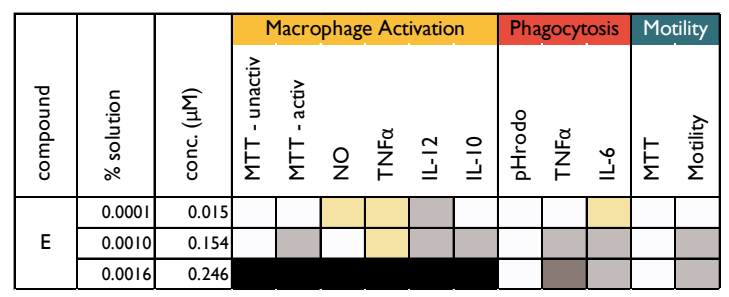

Figure 5.11. Activity profile for Compound E.

Categories are based on output levels compared to vehicle controls. Increases are represented in yellow, and decreases in grey. White is no change; black indicates no data were collected. Raw data can be found in appendix section 9.5.5.

Cytokine production by activated macrophages was altered by compound E, including elevated TNF $\alpha$, and NO production, though TNF $\alpha$ levels decreased during the phagocytosis assay. Compound E also reduced cell motility. Compound E suppressed IFN $\gamma$ production by T cells and IL-17A at lower concentrations. An increase was observed in IL-10 from B cells, however the increase was only apparent at a middle concentration and was associated with lower viability.
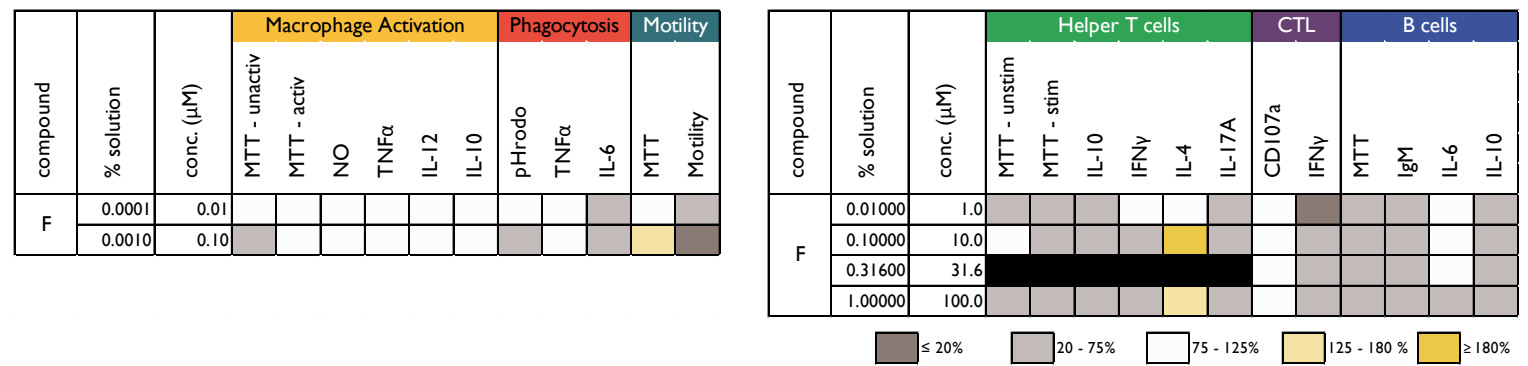

Figure 5.12. Activity profile for Compound F.

Categories are based on output levels compared to vehicle controls. Increases are represented in yellow, and decreases in grey. White is no change; black indicates no data were collected. Raw data can be found in appendix section 9.5.6.

The highest concentration of compound $F$ reduced the viability of unactivated macrophages, suggesting that compound $\mathrm{F}$ is cytotoxic to proliferating cells. Additionally, phagocytosis and cell motility were reduced. Interestingly, no decrease in viability or cytokine production was observed from LPS-activated macrophages. All tested concentrations were toxic to B and T cells, and because these aren't at the lowest possible concentration of $1 \mathrm{nM}$ it suggests this compound reduced splenocyte viability at all concentrations. 


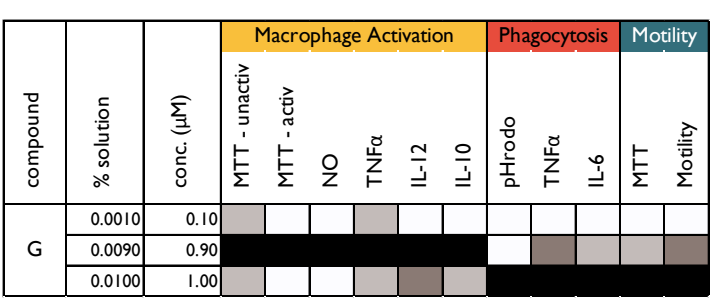

Figure 5.13. Activity profile for Compound G.

Categories are based on output levels compared to vehicle controls. Increases are represented in yellow, and decreases in grey. White is no change; black indicates no data were collected. Raw data can be found in appendix section 9.5.7.

As seen with the last compound, proliferating macrophages were less viable in the presence of compound G; an effect not seen in non-proliferative, activated macrophages. A reduction in TNF $\alpha$ production by macrophages and antibody production by $\mathrm{B}$ cells was seen with compound G. This compound enhanced IFN $\gamma$ production by stimulated T cells.
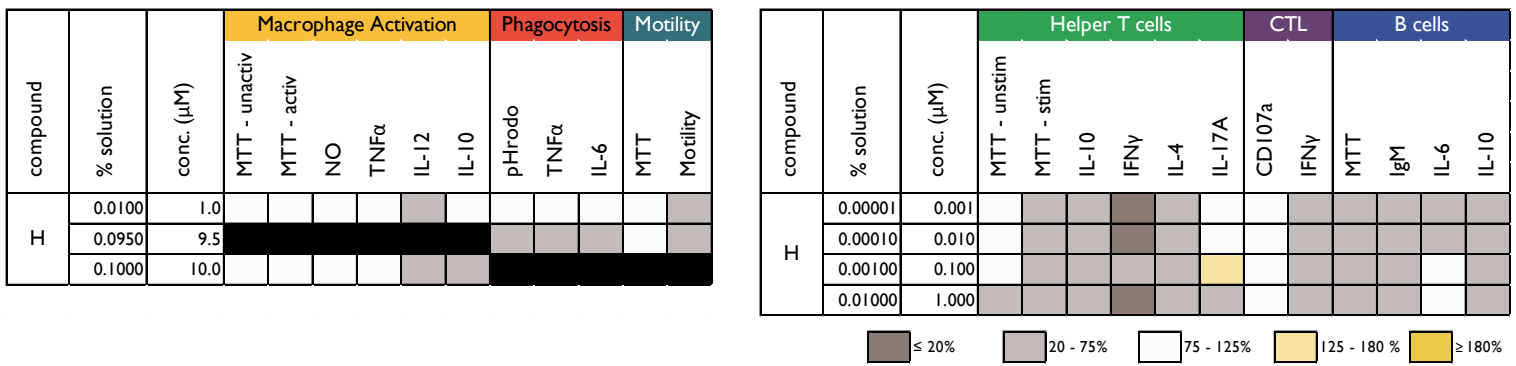

Figure 5.14. Activity profile for Compound $\mathrm{H}$.

Categories are based on output levels compared to vehicle controls. Increases are represented in yellow, and decreases in grey. White is no change; black indicates no data were collected. Raw data can be found in appendix section 9.5.8.

Compound $\mathrm{H}$ altered macrophage cytokines and at higher concentrations also decreased macrophage phagocytic ability and motility. Active $\mathrm{T}$ and $\mathrm{B}$ cells had greatly reduced viability, though resting splenocytes were not susceptible. 


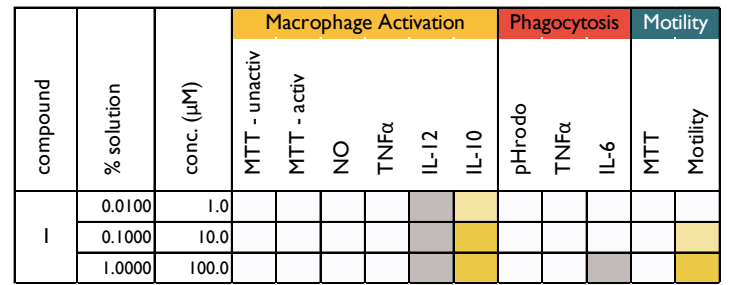

Figure 5.15. Activity profile for Compound I.

Categories are based on output levels compared to vehicle controls. Increases are represented in yellow, and decreases in grey. White is no change; black indicates no data were collected. Raw data can be found in appendix section 9.5.9.

Compound I showed little effect on immune cell viability. The most interesting effects observed were altered macrophage cytokines and the enhanced motility of macrophages in the presence of compound I. Treatment of $\mathrm{T}$ cells caused a generalised reduction in cytokine responses whereas B cells were unaffected by compound I.
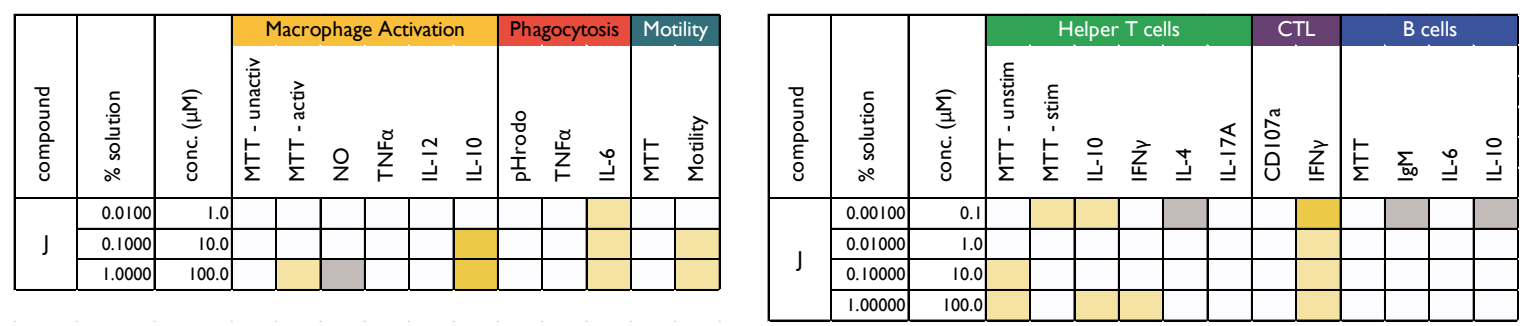

$\leq 20 \%$

$20-75 \%$

$75-125 \%$ $125-180 \% \square \geq 180 \%$

Figure 5.16. Activity profile for Compound J.

Categories are based on output levels compared to vehicle controls. Increases are represented in yellow, and decreases in grey. White is no change; black indicates no data were collected. Raw data can be found in appendix section 9.5.10.

Compound $\mathrm{J}$ showed stimulatory activity across a range of immune assays. This compound promoted macrophage motility and altered cytokine production; NO production is suppressed. T cells were also altered by compound J, with increased IL-10 and IFN $\gamma$ production, and decreased IL-4 with low concentrations of J. High concentrations also increased the metabolism of unstimulated splenocytes. At low concentrations of compound J, B cells had suppressed antibody and IL-10 production. 


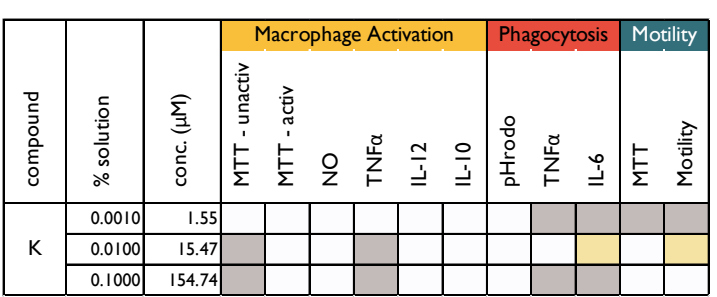

Figure 5.17. Activity profile for Compound K.

Categories are based on output levels compared to vehicle controls. Increases are represented in yellow, and decreases in grey. White is no change; black indicates no data were collected. Raw data can be found in appendix section 9.5.11.

Compound $\mathrm{K}$ showed variable activity across the immune assays. CTLs produced more IFN $\gamma$, whilst stimulated Th cells were relatively unaffected; activated $\mathrm{T}$ cells only have reduced viability at high concentrations of compound $\mathrm{K}$. B cell functions and macrophage motility changed depending on compound concentration; however, the most consistent result was a decrease in $\mathrm{TNF} \alpha$ production by macrophages in the activation and phagocytosis assays. Proliferating macrophages are negatively affected by compound K.
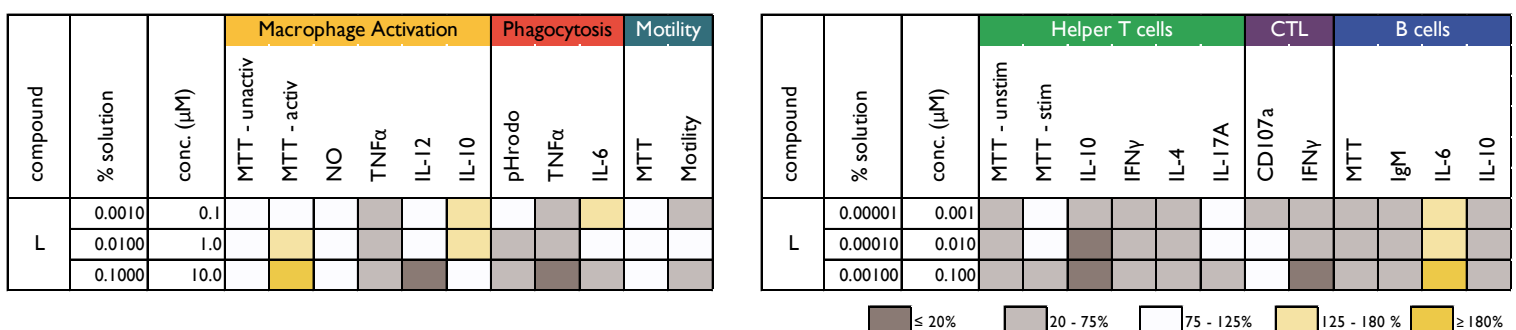

Figure 5.18. Activity profile for Compound $\mathrm{L}$.

Categories are based on output levels compared to vehicle controls. Increases are represented in yellow, and decreases in grey. White is no change; black indicates no data were collected. Raw data can be found in appendix section 9.5.12.

Compound L had a variety of effects on immune cells. Macrophage activation was altered, and the metabolism of activated macrophages increased. Both phagocytosis and TNF $\alpha$ production were reduced, as was cell motility. Compound L suppressed activities in B and T cells: B cells were suppressed through decreased viability, whilst activated T cells were viable but did not produce cytokines. Interestingly, unstimulated splenocytes succumbed to compound L. 
The immunomodulatory effects of the validation set compounds are summarised below. A wide range of activities were observed from compound screening, and this set of compounds saw changes to all immune activities assessed by this screening strategy. To determine if screening data are valid, this information was compared to published activities of these compounds. This is explored in the next section.

Table 5.3. Summary of effects seen from screening compounds in the validation set.

\begin{tabular}{|c|c|}
\hline Compound & Observed immunomodulatory effects \\
\hline A & $\begin{array}{l}\text { NO increased, TNF } \alpha \text { and IL- } 6 \text { increased during phagocytosis, suggesting a pro- } \\
\text { inflammatory state. IFN } \gamma \text { and IL-4 increased in T cells, IL-10 from B increased. }\end{array}$ \\
\hline B & $\begin{array}{l}\text { Phagocytosis and concurrent TNF } \alpha \text { increased. TNF } \alpha \text { down during activation. } \\
\text { Many T cytokines down, whilst CTL IFN } \gamma \text { increased. No change to B cells. }\end{array}$ \\
\hline $\mathrm{C}$ & $\begin{array}{l}\text { NO increased, other macrophage activation activity altered. Phagocytosis and TNF } \alpha \\
\text { down, also motility down, at high conc. IL-10 enhanced in T and B cells. }\end{array}$ \\
\hline $\mathrm{D}$ & $\begin{array}{l}\text { Most cytokines suppressed, except TNF } \alpha \text { and NO which increased. Phagocytic activity } \\
\text { down. Motility increased with low conc., suppressed (with viability) at higher conc. } \\
\text { Degranulation enhanced; CTL IFN } \gamma \text { down. Lower conc. increases IL-4 and IFN } \gamma \text {. }\end{array}$ \\
\hline $\mathrm{E}$ & $\begin{array}{l}\text { Cytokine suppression in macrophages (except TNF } \alpha \text {, NO). IFN } \gamma \text { suppression in } \mathrm{T} \text { and } \\
\text { CTL. Motility decreased. B cells largely unaffected. }\end{array}$ \\
\hline $\mathrm{F}$ & $\begin{array}{l}\text { Anti-proliferative: only unstimulated RAW } 264.7 \text { cells succumb. Phagocytosis and cell } \\
\text { motility decreased. Toxic to T and B cell populations. }\end{array}$ \\
\hline G & $\begin{array}{l}\text { Anti-proliferative; decreased TNF } \alpha \text { and IL-6. B cell IgM decreased. } \\
\text { IFN } \gamma \text { up in T and CTL assays. }\end{array}$ \\
\hline $\mathrm{H}$ & $\begin{array}{l}\text { Motility, phagocytosis (incl. cytokines) suppressed. } \\
\text { Activated macrophages largely unaltered. } \\
\text { Active T cell and B cell viability suppressed, resting cells unaffected. }\end{array}$ \\
\hline I & $\begin{array}{l}\text { Enhanced motility, macrophage cytokines (IL-10 and IL-12) altered. } \\
\text { T cell cytokines suppressed, B cells unaffected }\end{array}$ \\
\hline $\mathrm{J}$ & $\begin{array}{l}\text { NO suppressed; changes to macrophage IL-10. Increased IL-6 during phagocytosis. } \\
\text { IFN } \gamma \text { and IL-10 up, and IL-4 down, in T cells. IgM and IL-10 from B decreased. }\end{array}$ \\
\hline K & $\begin{array}{l}\text { Anti-proliferative. TNF } \alpha \text { down, and IL-6 variable. Enhanced motility. } \\
\text { Stimulated T cells viability affected at high conc., concentration-dependent decreases to } \\
\text { IL-4 and IL-17A. Variable effects on IL-10 and IgM from B cells. CTL IFN } \gamma \text { enhanced }\end{array}$ \\
\hline $\mathrm{L}$ & $\begin{array}{l}\mathrm{TNF} \alpha \text {, phagocytosis and motility decreased in macrophages, other cytokine changes are } \\
\text { variable. Resting T cell viability decreased. Activated T cells viable, though cytokines } \\
\text { are suppressed. B cells not viable. CTL activities decreased. }\end{array}$ \\
\hline
\end{tabular}




\subsubsection{Screening data can adequately identify immunomodulatory activity}

To test the screening strategy's ability to adequately capture the immunomodulatory properties of compounds, compounds with known effects on the immune system were coded by a co-worker and tested. After using the screening strategy to generate activity profiles, the major immune modifying effects of the compounds were summarised (Table 5.3). Through matching this information to what is published about the immunomodulatory effects of the compounds (Table 5.4), an attempt was made to identify the tested compounds. If successful, it demonstrates that data generated from this screening strategy is valid and, in a round-about way, matches independent observations.

Table 5.4. Expected activities of validation set compounds based on observations from literature

\begin{tabular}{|c|c|c|}
\hline Compound & Immunomodulatory effects & Ref. \\
\hline Aminoguanidine & NO suppression. & [154] \\
\hline BPS & $\begin{array}{l}\text { Induces altered macrophage: increases NO, reduces TNF } \alpha \text { and IL-6. } \\
\text { Iron deficiency causes pro-inflammatory T cell bias in vivo. }\end{array}$ & $\begin{array}{l}{[155-} \\
159]\end{array}$ \\
\hline Brefeldin A & $\begin{array}{l}\text { Cytokine suppression. Cytoskeletal alteration with longer treatment } \\
\text { (motility decreases with extended treatment). }\end{array}$ & $\begin{array}{l}{[160-} \\
162]\end{array}$ \\
\hline Chloramphenicol & $\begin{array}{l}\text { Reduces proliferation through mitochondrial damage. No effect on } \\
\text { NO production. Suppresses T cell activation. }\end{array}$ & $\begin{array}{l}{[163-} \\
166]\end{array}$ \\
\hline Dopamine & $\begin{array}{l}\text { Decreases phagocytosis, TNF } \alpha \text {, and other cytokines. Motility } \\
\text { decreases in neutrophils. Increases IL-10 in many cells, and IL- } 4 \text { in } \\
\text { T cells. }\end{array}$ & $\begin{array}{l}{[167-} \\
171]\end{array}$ \\
\hline Doxorubicin & Anti-proliferative. IFN $\gamma$ increases in $\mathrm{T}$ cells. & {$[172,173]$} \\
\hline Gliotoxin & $\begin{array}{l}\text { Decreases TNF } \alpha \text {, IFN } \gamma, \text { NO, and IL-12 from macrophages. } \\
\text { Decreases motility. Supresses IFN } \gamma \text { production, and CTL activity. }\end{array}$ & {$[174,175]$} \\
\hline Ibrutinib & $\begin{array}{l}\text { B cell suppression. Alters/inhibits macrophage activation. T cells } \\
\text { altered. }\end{array}$ & $\begin{array}{l}{[176-} \\
180]\end{array}$ \\
\hline Ibuprofen & $\begin{array}{l}\text { T cell suppression. Alters macrophage IL-10 and IL-12 production. } \\
\text { Cell motility enhanced, assumed through PGE2 inhibition. } \\
\text { Antibody production reduced in human B cells }\end{array}$ & $\begin{array}{l}{[181-} \\
186]\end{array}$ \\
\hline Ixabepilone & $\begin{array}{l}\text { Anti-proliferative, reduce cell motility though microtubule } \\
\text { stabilisation. }\end{array}$ & {$[187,188]$} \\
\hline Rapamycin & $\begin{array}{l}\text { Active T cells succumb. Inhibits phagocytosis. B cell activity } \\
\text { decreases. }\end{array}$ & $\begin{array}{l}{[189-} \\
191]\end{array}$ \\
\hline Sulpiride & Decreases in $\mathrm{TNF} \alpha, \mathrm{NO}$. & {$[170]$} \\
\hline
\end{tabular}


Overall, identifying the compounds was successful, though some were difficult to identify due to the inability to find relevant data about their immunomodulatory activities in the literature. Compounds were identified based on similarities between experimental data and the literature (i.e. Table 5.3 and Table 5.4) and through a process of elimination. Side-byside comparisons of immunomodulatory activity were difficult. The breadth of information about compound activity in the literature did not match what was generated from the screening.

F, G, K are anti-proliferative compounds, as determined by their effects on unstimulated RAW 264.7 cells. These activities are associated with Chloramphenicol, Doxorubicin, and Ixabepilone which can reduce proliferation regardless of cell type - other compounds in this list inhibit T cell proliferation through T cell-specific mechanisms. Ixabepilone inhibits cell motility and, of these three compounds, is seen with compound F; F also has the most similar profile to paclitaxel, tested in the last chapter. Compounds $\mathrm{G}$ and $\mathrm{K}$ are similar, with the difference here being their effects on T cells different activity states. Compound $\mathrm{K}$ affects active $\mathrm{T}$ cells, whereas $\mathrm{G}$ affects $\mathrm{T}$ cell viability similarly in both resting and active states. This difference suggested compound $\mathrm{K}$ was Chloramphenicol, and for $\mathrm{G}$ to be Doxorubicin. The increased IFN $\gamma$ with compound G treatment supported this deduction.

B cell suppressants include compounds $\mathrm{H}$ and $\mathrm{L}$, which are either Rapamycin or Ibrutinib. The difference between these two compounds are their effects on T cells during different states of activation. Compound $\mathrm{L}$ affects resting splenocytes and has little effect on active T cells, whilst the opposite is seen with compound H. This suggests that Rapamycin is compound $\mathrm{H}$ whilst $\mathrm{L}$ is Ibrutinib. This suggestion is supported by the effects on macrophage activities: Ibrutinib is known to alter macrophage activation which is seen with $\mathrm{L}$ more so than $\mathrm{H}$.

Widespread cytokine suppression is seen with compound $\mathrm{D}$, which resembles the activity of Brefeldin A, and the changes to motility also matched published data about Brefeldin A. Gliotoxin best matched compound E. Compound E suppresses IFN $\gamma$ production, a known activity of Gliotoxin, which is a unique feature amongst the remaining unidentified compounds. The remaining compounds were more difficult to match, especially as relevant literature was difficult to source - many observations on immune cell activities are from other species, or other cell types. Dopamine is known to increase IL-10 production 
in many cell types, which is seen with compound C treatment. Although IL-10 changes are seen with some of the other remaining coded compounds, the decreased cell motility of Dopamine treatment is only notable with compound C.

Compounds A, B, I and J remain to be matched to AMG, BPS, Ibuprofen and Sulpiride. Ibuprofen is associated with T cell suppression, which is observed with compounds B and I. Because Ibuprofen is known to alter IL-10 and IL-12 production by activated macrophages, it suggested compound I is this NSAID; this is supported by the expected enhancement of cell motility seen with compound I. With little confidence, compound A was determined to be BPS, relying on the increase of pro-inflammatory IFN $\gamma$ matching the expected pro-inflammatory effect of BPS, and the expected increase in NO production. Sulpiride and AMG were hard to identify as there was little relevant information in the literature about the activity of these compounds. Both compounds shared similar activities, so an identification could not be made with confidence. It was later revealed that AMG was compound J, and Sulpiride was compound B. AMG indeed reduced NO production as expected, though Sulpiride did not. These compounds were found to have other interesting effects that were not found during the literature search.

After predicting the identity of the blinded compounds using the outcomes from screening, the true identity of the compounds was revealed. With the exception of the last two compounds, all were identified successfully. This exercise highlighted some limitations of compound testing: variability in assay design alters what is understood about compound activity. For example, decreases in NO production are expected with three compounds based on literature (Gliotoxin, AMG and Sulpiride), however decreases were only seen with compound J, possibly due to differences in assay design. Conservatively-placed thresholds could have explained these disagreeing observations, however NO decreases by these compounds were not evident in the raw data. Despite this, sufficient data were generated to characterise twelve compounds to differentiate and subsequently identify ten majority of them. Additionally, these data reflect independent findings. The observed and published activities of the compounds are summarised in Table 5.5. 


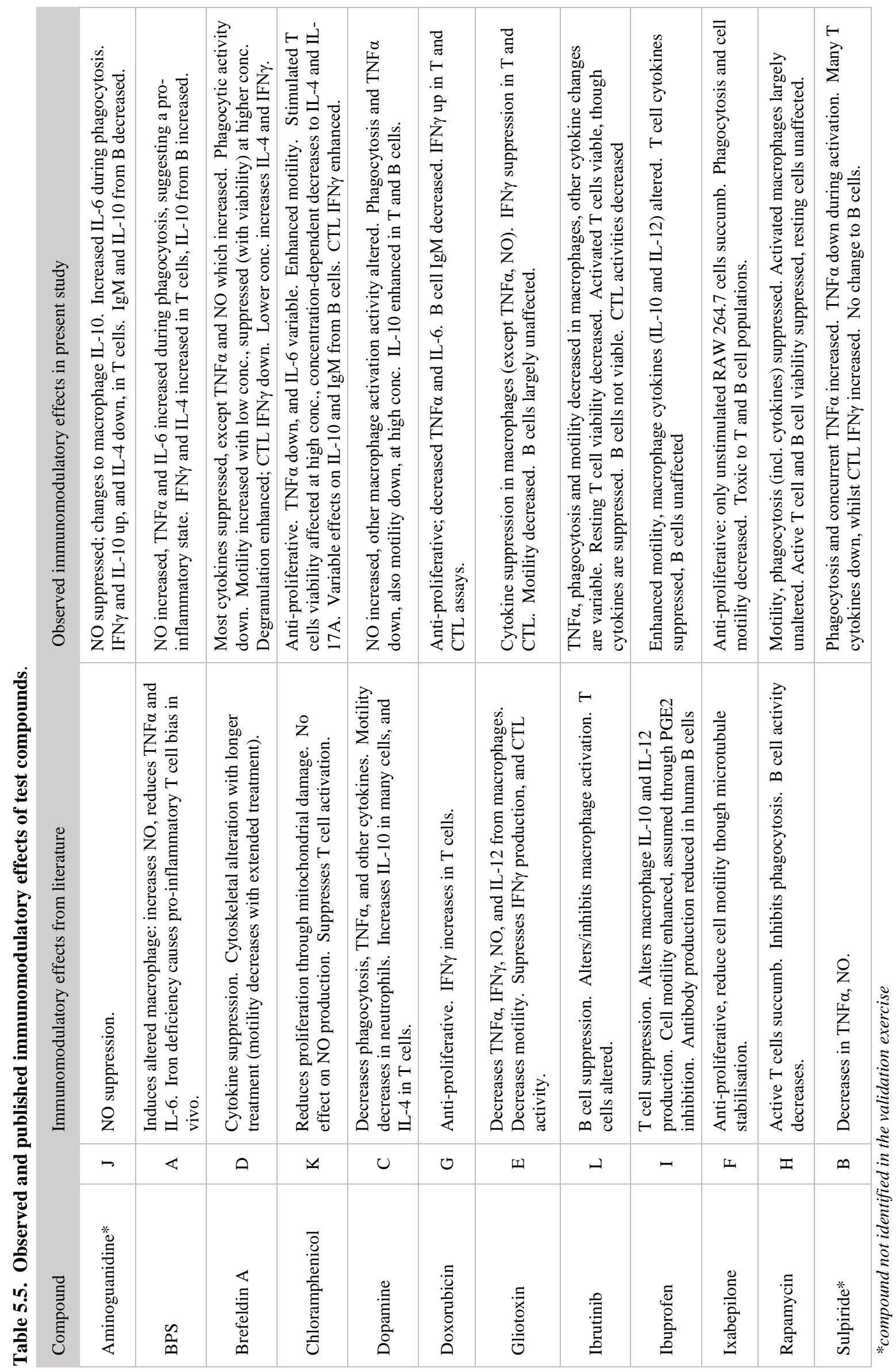




\subsection{Discussion}

In this chapter the screening strategy was shown capable of identifying the immunomodulatory activity of test compounds. The screening strategy operated well after making an amendment to the concentration identification stage of the workflow. Many compounds did not alter cell metabolism, previously identified as a means to narrow down concentrations of interest, so additional data were collected from the first assays. Cytokine data from both the macrophage and helper $\mathrm{T}$ cell activation assays provided insight into the concentrations of test compounds that should be further tested by identifying bioactivity. This amendment was successful: the testing concentrations that were selected by this method generated sufficient data to characterise the compounds and these data matched independent observations.

Compounds were tested blind which drove the fine-tuning of the initial steps of the screening strategy. This mimics working with novel compounds where little or no information about the bioactive concentration range would be available. Standardising the initial compound titration to identify bioactive concentrations of interest removes the need to know the bioactive concentrations of compounds prior to screening. It also made for efficient compound preparation: the compound dilutions during this step were consistent for each compound, unlike in the previous chapter, which saved time and made for simple experimental set-up for the first two assays.

Cytotoxicity dose-response curves were generated for each cell type, where possible, from these data. $\mathrm{IC}_{20}$ concentrations were calculated for later use through the remaining assays. Using the $\mathrm{IC}_{20}$ concentrations alongside other similar concentrations was done to assess the utility of using $\mathrm{IC}_{20}$ concentrations to identify high, non-toxic compound concentrations. After looking at the generated data, testing the $\mathrm{IC}_{20}$ concentrations of compounds was not especially informative. The data captured here was also captured with the nearest log-fold concentration tested. To clarify, compound G was tested at $0.01 \mu \mathrm{M}$, as well the $\mathrm{IC}_{20}$ value of $0.009 \mu \mathrm{M}$ however the immunomodulatory effects of compound $\mathrm{J}$ across these two concentrations was the same. This was also observed with compounds D, E and F. Compound $\mathrm{H}$ was tested at the $\mathrm{IC}_{20}$ of $0.095 \mu \mathrm{M}$ but it would have been more convenient to prepare $0.1 \mu \mathrm{M}$ treatments which would have likely elicited the same effects. Instead of $\mathrm{IC}_{20}$ values, simply using the highest log-fold concentration of compound before 
cell viability drops below 75\% (of vehicle control) provide sufficient data. In most cases this would include the $\mathrm{IC}_{20}$. It avoids the need of preparing 'bespoke' treatments of particular compounds which added time to the compound-preparation stages of the assaying process. Additionally, compound-induced changes to macrophage activation and helper $\mathrm{T}$ cell activity are not collected at the $\mathrm{IC}_{20}$ concentration and to do this would require an additional run of these assays.

The rest of the screening process went smoothly, and no additional changes were necessary. After narrowing down compound concentrations, three 96-well plates were needed to test compounds through each assay. Assuming an average molecular mass of 500 Da only $0.5 \mathrm{mg}$ of each compound was required for one run through the screen and, in optimal situations, one person can complete the screen in four weeks if testing up to thirty compounds. The activity profiles were, again, a useful way to interpret the data from screening compounds in this chapter. These were used to successfully identify unknown compounds, and the activities matched what is reported in the literature about the compounds. In other words, the profiles summarise data well, and there is congruence between the generated screening data, and data from independent testing.

The value of measuring NO from activated macrophages was previously questioned as NO production was not altered by any compound in the previous chapter. Several compounds used in this chapter altered this activity and justifies its inclusion in the array of measured assay outputs. It was useful to identify coded compounds, and this approach could be used to identify changes to bactericidal activity of macrophages. Similarly, in the previous chapter, it was observed that the degranulation assay tolerates and may require higher concentrations of compounds to modify this process. By using compounds at concentrations that are non-toxic to helper $\mathrm{T}$ cells during activation, it implies that these concentrations would not interfere with the activation of naïve CTLs and therefore can be used to assess for effects on degranulation without having to repeatedly look for $\mathrm{T}$ cell activation inhibition on the two subsets of $\mathrm{T}$ cells. Though data in this chapter show changes to CTL degranulation, there is still a risk that false-negative results are being produced by using too low a concentration of compounds. This still remains one limitation in the screening strategy and a series of experiments need to investigate and address this problem. 
Overall, the screening strategy performed well and offers a methodology by which compounds can be screened for immunomodulatory activity. No prior knowledge of compounds is required as this strategy includes a step to identify bioactivity, and the concentrations of compounds where bioactivity occurs. In the short space of a month, the immunomodulation by test compounds can be tested across four different immune cell types which together represent many cellular processes used by the immune system. The strategy does not extensively investigate these changes, but serves as a means to identify compounds that have immunomodulatory effects which can then be further investigated.

\subsection{Summary}

In this chapter the screening strategy's ability to characterise the immunomodulatory activity of compounds was validated. Known immunomodulators were screened and ten out of twelve deidentified compounds were discovered. The data generated by the screen reflected activities reported by independent studies allowing for the discovery of the deidentified compound set. The strategy required an amendment to the step where testing concentrations were identified, including the analysis of cytokine data from the initial assays to assist in concentration identification when cell viability was not affected by test compounds. With confidence in the effectiveness of the screening strategy, novel compounds can be screened. Their immunomodulatory activities are described in the next chapter. 
Chapter 6:

Novel immunomodulators 


\subsection{Introduction}

This chapter will investigate the immunomodulatory activities of both novel compounds (e.g. Samoan natural plant products, a metabolic inhibitor, and a heparan sulfate mimetic) and clinically available drugs with known non-immune targets (i.e. neuroreceptors). The first group of novel compounds are aqueous and methanolic extracts from Psychotria insularum, which are under investigation by the VUW Chemical Genetics and Natural Products research groups. P. insularum is a Samoan medicinal plant used to reduce swelling, and so was screened for potential immunomodulatory effects[192]. The second compound is MTDIA (Methylthio-DADMe-Immucillin A), also under investigation by the VUW Chemical Genetics lab for its anti-cancer activity, which has previously described immunomodulatory properties[193]. This inhibitor of MTA (Methylthioadenosine) metabolism is said to reduce TNF $\alpha$ due to MTA accumulation, prompting an investigation into the other effects MTDIA has on the immune system[194-196]. The third novel compound that was investigated is a heparan sulfate (HS) mimetic. Heparan sulfates are known to inhibit inflammation by reducing the ability of leucocytes to migrate through the body[197,198]. HS mimetics target the enzyme 154eparinase which is important for tumour metastasis and is involved with establishing inflammation[199]. The HS mimetic HS16-38 was provided by the Ferrier Research Institute to investigate the immunomodulatory effects of this synthetic analogue. Additionally, three neuroreceptortargeting drugs, two of which are used clinically, were screened for immunomodulatory effects. Finally, small quantities of Hamigeran $\mathrm{G}$ and Peloruside A were also available and were tested through the initial stage of the screening strategy. As in the previous chapters, individual activity profiles were created to visualise the pattern of bioactivity of each compound. After assessing each set of compounds individually, a hierarchical cluster using data from all the compounds tested so far was generated to explore whether the pattern of effects could offer insight into compound activity. 


\subsection{Aims}

Previous chapters have focused on developing and validating a screening strategy using compounds with known targets and whose bioactivity is supported by numerous studies. However, this screening strategy is intended to identify novel compounds for whom little biological information may be available. Therefore, this chapter investigates the utility of the proposed screening strategy by testing a range of compounds, from those that are completely novel to others in current clinical use. Additionally, because the targets are unknown, this chapter explores whether hierarchical cluster analysis of the whole compound data set from this work can provide insight into the molecular pathways targeted by each compound. In summary, this chapter has two objectives:

1. Describe the immunomodulatory effects of novel compounds

2. Explore the utility of a cluster analysis of screening data 


\subsection{Results}

Novel compounds were screened according to the workflow previously described (refer last chapter). Concentrations for testing were identified as done previously. These data, and screening data can be found in appendix section 9.6, (pg 234). Concentrations, unless otherwise stated, were identified as in section 5.3.1 (pg 130), and the activity profiles presented in this section were generated as done previously (section 4.3.10, pg 114).

\subsubsection{Immunomodulation by Psychotria insularum, a medicinal plant}

Many bioactive compounds are found in nature, many of which have become drugs due to their therapeutic activities[200,201]. P. insularum is a plant used in traditional Samoan medicine for a variety of ailments, including inflammation[202]. It has also been shown previously to inhibit inflammatory prostaglandin biosynthesis[192,203]. In collaboration with the Chemical Genetics lab at VUW, P. insularum was investigated for its immunomodulatory effects.

Aqueous and methanolic extracts were supplied by the collaborators. Aqueous extracts were made by washing leaves of the plant, and after homogenisation through a juicer, the liquid portion was filtered for use. The methanolic extract was made from steeping the solid material from juicing in methanol overnight. Solids were then removed, and the methanol was evaporated from samples which were then reconstituted in DMSO[192]. In general, aqueous extracts contain water-soluble, polar molecules, whilst methanolic extracts contain non-polar compounds from source material. These two types of extract were screened through the assays to identify the immunomodulatory activity of $P$. insularum. 
All immune cell types were altered by the aqueous $P$. insularum extract, and nearly every assay output was altered. Macrophages had altered cytokine production and decreased phagocytic ability and motility. Generally, B and T cell activities were suppressed by this extract, except for IL-10 production which was enhanced in both T and B cells. This suggests the aqueous extract of $P$. insularum has an anti-inflammatory effect on the adaptive immune system. This is supported by the observed decrease of IFN $\gamma$ and IL-17A production. Note that concentrations of this aqueous extract were selected by a collaborator after conducting yeast-based screens. These concentrations were confirmed to be suitable in initial macrophage activation and helper $\mathrm{T}$ cell assays, and were deemed suitable; therefore the concentration selection method was not used for these extracts.
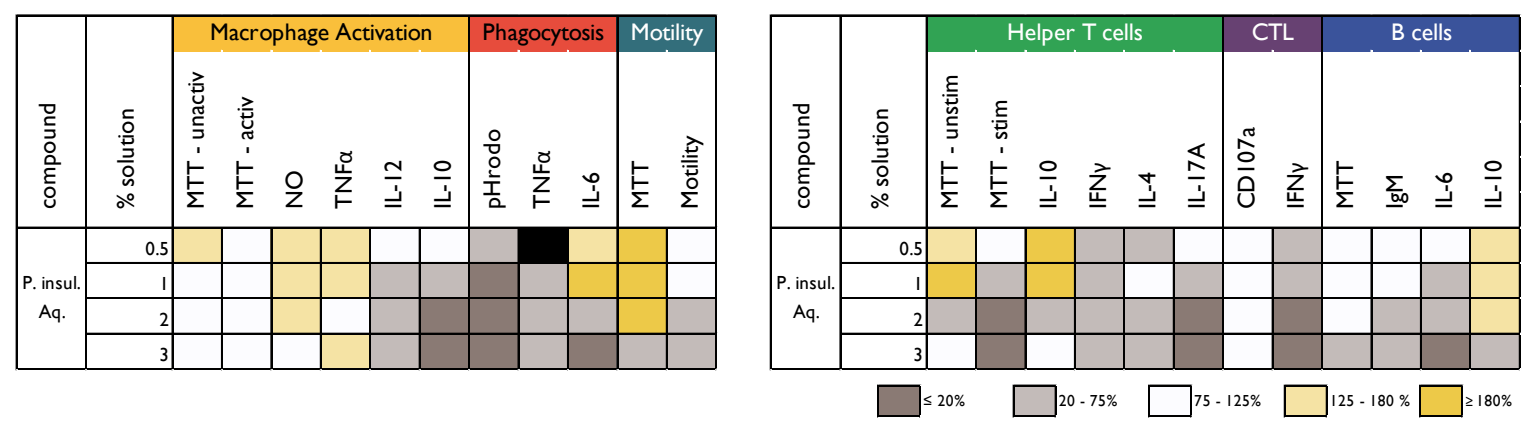

Figure 6.1. Activity profile of the aqueous extract of $P$. insularum.

Categories are based on output levels compared to vehicle controls. Data are from each assay conducted once, in duplicate. Raw data can be found in appendix section 9.6.1. 
The extract's effects on macrophage activation were investigated further using BMDM. IL-10 increased, and IL-12 decreased, across all tested concentrations of the extract. TNF $\alpha$ levels also increased, while NO production and viability were unaltered by treatment. The largest effect was seen with a $2 \%$ solution of aqueous P. insularum (Figure 6.2, A). Neutralising antibodies or recombinant cytokines were also added alongside treatments during macrophage activation to investigate how the anti-inflammatory effects of the extract were induced; these additions did not reduce macrophage viability (Figure 6.2, B). When IL-10 is neutralised in the system by antibody, the IL-12 production by vehicletreated macrophages was enhanced compared to IgG isotype control. However, extracttreated cells had decreased IL-12 levels compared to vehicle controls, suggesting the extract affects IL-12 production independently of IL-10 alteration (Figure 6.2, C). This extract also increased IL-10 levels compared to vehicle-treated controls even when IL-12 was altered in the system (Figure 6.2, D). In macrophages, the anti-inflammatory effects of this extract were caused by IL-12 suppression and IL-10 enhancement independently.
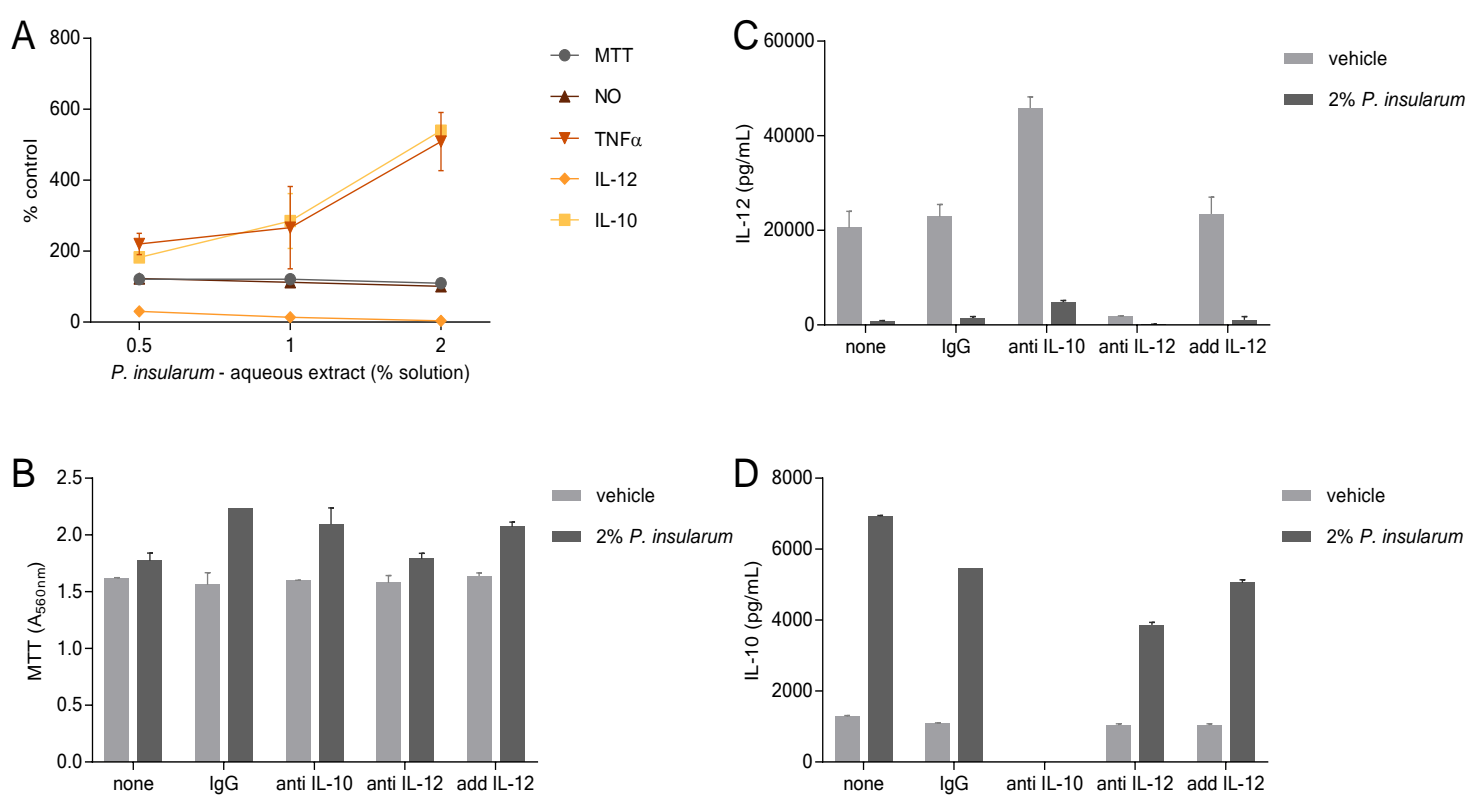

Figure 6.2. $P$. insularum alters IL-10 and IL-12 production independently of each other

(A). 100,000 BMDM were plated into a 96-well plate and primed for $18 \mathrm{~h}$ with $20 \mathrm{U} / \mathrm{mL}$ IFN $\gamma$. Indicated concentrations of aqueous $P$. insularum, or vehicle controls, were added to wells alongside $200 \mathrm{ng} / \mathrm{mL} \mathrm{LPS}$. After $24 \mathrm{~h}$, cell viability was assessed by MTT, and cytokines were measured from cell supernatants.

(B) 100,000 BMDM were seeded into wells of a 96-well plate and primed for $18 \mathrm{~h}$ with $20 \mathrm{U} / \mathrm{mL} \mathrm{IFN \gamma .2 \%}$ aqueous $P$. insularum, or vehicle controls, was added to wells alongside $200 \mathrm{ng} / \mathrm{mL} \mathrm{LPS}$. At the same time, either $5 \mathrm{ng} / \mathrm{mL}$ recombinant murine cytokines, or $2 \mu \mathrm{g} / \mathrm{mL}$ cytokine-neutralising antibodies were added to wells. IgG served as a control to identify changes to macrophage activation in the presence of antibody. After 24 h, cells were subjected to MTT (B), and IL-12 (C) and IL-10 (D) were measured from supernatants. Data are means and SEM each from one experiment conducted in duplicate. 
In comparison to the aqueous extract, the methanolic extract of P. insularum had slightly different effects on immune cell processes. Generally, immune cell activities were suppressed. TNF $\alpha$ production by macrophages was reduced, and other cytokines were altered. Phagocytosis and concurrent cytokine production were also reduced; however, cell motility was enhanced. Inflammatory IFN $\gamma$ and IL-17a production were suppressed in helper T cells, and whilst IL-4 was also suppressed, regulatory IL-10 production was unaffected. At lower concentrations, T cell cytokines were generally unchanged, although an enhancement of IL-10 was observed. B cell IgM and IL-10 production was suppressed, as well as the IFN $\gamma$ from degranulating CTLs, however degranulation itself is enhanced.
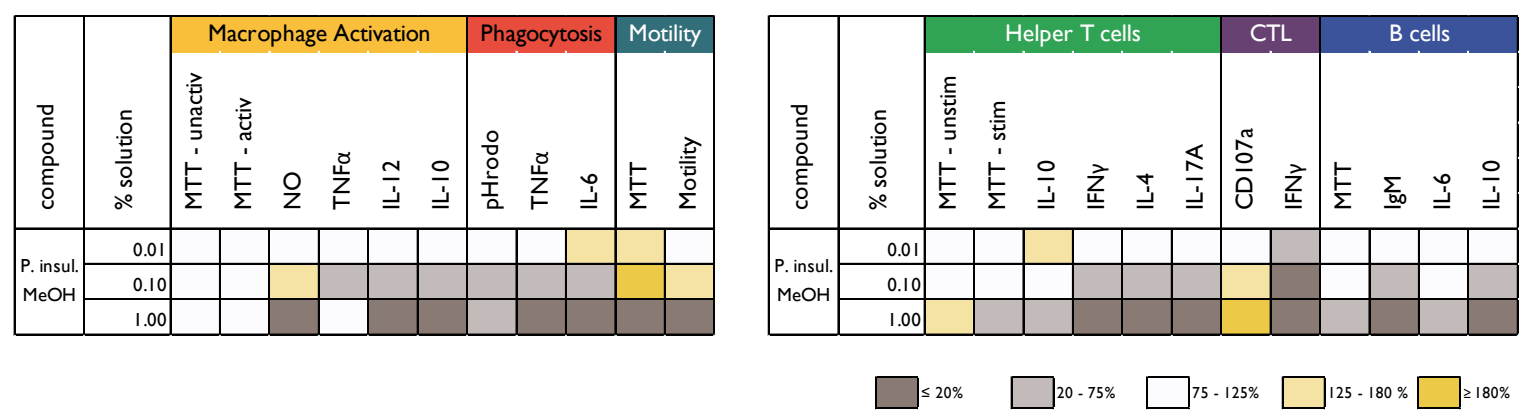

Figure 6.3. Activity profile of the methanolic extract of $P$. insularum

Categories are based on output levels compared to vehicle controls. Data are from each assay conducted once, in duplicate. Raw data can be found in appendix section 9.6.2.

In summary, $P$. insularum contains compounds that can elicit an anti-inflammatory effect on the immune system: many activities were supressed, however IL-10 production by many cells was enhanced. Pro-inflammatory mediators, such as IFN $\gamma$ and IL-17A, were reduced which further enhances the anti-inflammatory effects of $P$. insularum. There are differences between the aqueous and methanolic extracts. The former enhances TNF $\alpha$ produced by activated macrophages and IL-10 from B cells, whilst the latter suppresses TNF $\alpha$, enhances cell motility and enhances CTL degranulation. Testing both these extracts in vivo with a model of infection that involves both the innate and adaptive immune system could identify the implications of these differences. It would address the elevated TNF $\alpha$ level's impact on inflammation resolution, or the effects of enhanced CTL degranulation. Further work to isolate the bioactive components of these $P$. insularum extracts is currently underway. 


\subsubsection{Immunomodulation by MTDIA}

MTDIA (Methylthio-DADMe-Immucillin A) is an MTAP (Methylthioadenosine phosphorylase) inhibitor and therefore blocks MTA (Methylthioadenosine) metabolism. Cellular proliferation is inhibited when MTA accumulates, making MTDIA a potential treatment for cancer[204,205]. MTA accumulation is associated with T cell suppression, therefore MTDIA was screened to observe this, and any other immunomodulatory effects[194,206].

MTDIA had very few effects on macrophages, though it suppressed $\mathrm{TNF} \alpha$, which is a known outcome of MTA accumulation[196]. A higher concentration decreased IL-6 production, and increased cell motility. Lymphocyte processes appear more sensitive to MTDIA treatment. The expected T cell suppression is observed through reduced viability which is associated with the reduced production of all cytokines. Decreased IgM and IL-10 production was seen in B cells, and decreased IFN $\gamma$ production from CTLs was also seen with MTDIA treatment.

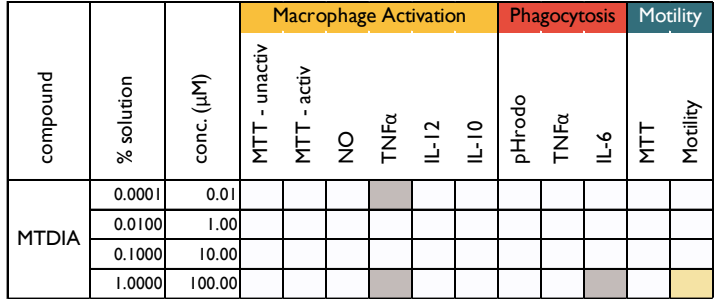

Figure 6.4. Activity profile of MTDIA

Categories are based on output levels compared to vehicle controls. Data are from each assay conducted once, in duplicate. Raw data can be found in appendix section 9.6.3

To exaggerate the activity of MTDIA, a modified macrophage activation assay was run. In this iteration, an additional $25 \mu \mathrm{M}$ MTA was added to wells during MTDIA treatment, and the same assay outputs were measured (Figure 6.5). With this design, MTA accumulation is caused both by inhibited MTA metabolism endogenously, and through exogenous MTA. The viability of cells was not affected by the addition of MTA and MTDIA (see section 9.6.3.3, pg 239239). Compared to untreated cells (solid black), NO production increased when MTA was added to wells (solid blue), and adding MTDIA treatment reduced this elevated NO (striped blue); the compound's effect was not obvious when MTA is not used (striped black, Figure 6.5, A). Additional MTA did not change IL-12 production, but 
MTDIA treatment increased levels of this cytokine (Figure 6.5, B). The decrease in TNF $\alpha$ production by MTDIA was seen with and without MTA, however additional MTA exaggerated TNF $\alpha$ suppression (Figure 6.5, C). MTDIA had no effect on IL-10 production regardless of MTA levels (Figure 6.5, D). Some of the observations seen in the MTA-free conditions differed from the screening data, possibly due to the different batch of MTDIA used.

To summarise, MTDIA suppressed lymphocyte activities, and interfered with active T cell viability. Activated macrophages were also altered with MTDIA, though these cells may not be as sensitive to its effects. The macrophage experiments should be repeated with BMDM to confirm activity. This information is useful for understanding the potential side effects that MTDIA may have on the immune system should it be pursued as an anti-cancer therapy.
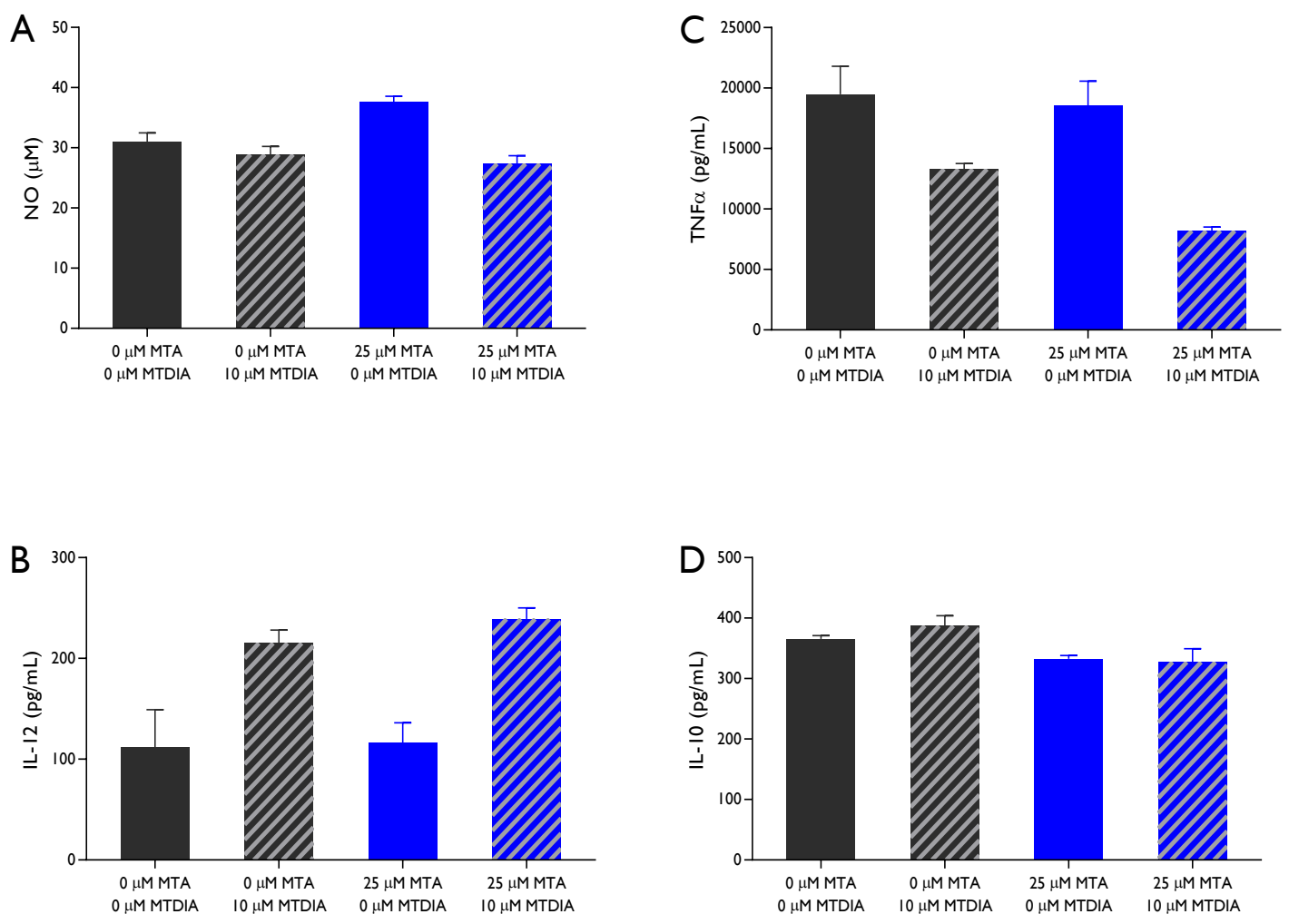

Figure 6.5. Additional MTA during macrophage activation exaggerates TNF $\alpha$ suppression by MTDIA 50,000 RAW 264.7 cells were seeded into wells and primed with $20 \mathrm{U} / \mathrm{mL}$ IFN $\gamma$ for $18 \mathrm{~h}$. Cells were activated with $200 \mathrm{ng} / \mathrm{mL}$ LPS, and either $25 \mu \mathrm{M}$ MTA, $10 \mu \mathrm{M}$ MTDIA, both or vehicle controls were added to wells. After 24 h, cell viability was assessed by MTT (see section 9.6.3.3, pg 239), and NO (A), IL-12 (B), TNF $\alpha$ (C) and IL-10 (D) were measured from cell supernatants. Data are means and SEM from one experiment conducted in duplicate. 


\subsubsection{Immunomodulation by HS $16-38$, a Heparan sulfate mimetic}

Heparan sulfates are touted for their ability to inhibit cancer metastasis and to alter chemokine production which led us to test a HS mimetic synthesised by the Ferrier Research Institute[197,198]. HS16-38 suppressed cell motility when macrophages were treated with relatively high concentrations of the compound. At this same concentration phagocytosis was also suppressed, though increased TNF $\alpha$ production during the longer macrophage activation assay was observed. At lower concentrations, the only changes to macrophages were seen during the phagocytosis assay: phagocytosis itself was unaffected whilst TNF $\alpha$ and IL-6 production decreased. T cells and B cells are altered in different ways by HS16-38. IFN $\gamma$ from CTLs, and B cell cytokine and IgM production, were suppressed whilst helper T cell cytokine production was generally enhanced.
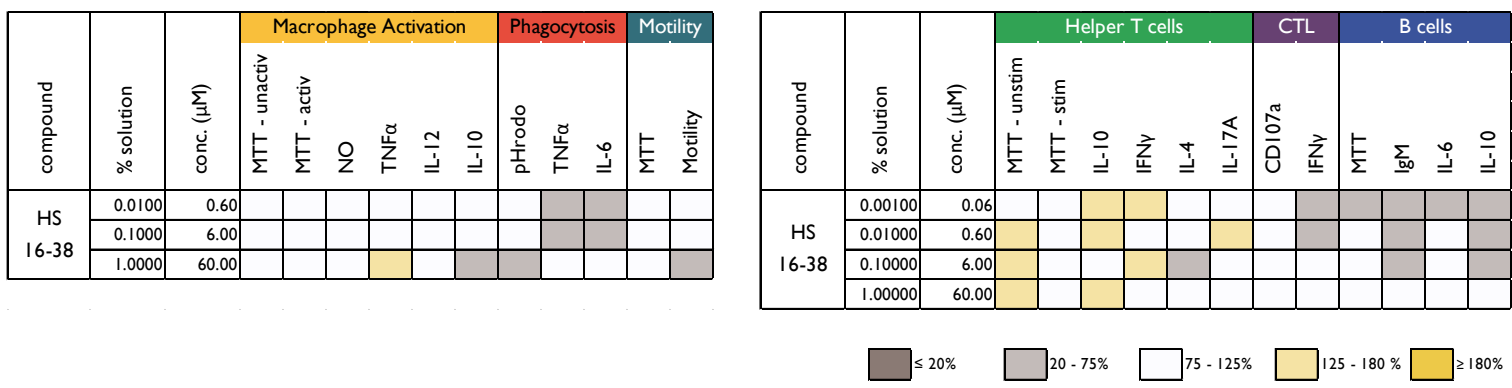
$125-180 \%$

Figure 6.6. Activity profile of the Heparan sulfate mimetic HS16-38

Categories are based on output levels compared to vehicle controls. Data are from each assay conducted once, in duplicate. Raw data can be found in appendix section 9.6.4.

HS16-38 may be of interest when seeking ways to manage B cell or macrophage dysregulation, but the enhanced T cell activity may cause unwanted effects. As HS16-38 increased helper $\mathrm{T}$ cell cytokine production and also the metabolic activity of unstimulated splenocytes, it would be worthwhile to investigate any inherent stimulatory activity of the HS mimetic on T cells. 


\subsubsection{Immunomodulation by neurotransmitter receptor-targeting compounds}

After recent research into the role of dopamine neurotransmitters in the immune system, our group’s research interests have recently included the immune system's interaction with additional neurotransmitters. Compounds NTR1, NTR2 and NTR3 each have varying activity, but all target the same neurotransmitter system, and these were screened to identify their effects on immune cell processes. Two of these compounds are drugs which are currently used for non-immune applications, whilst the third is currently under investigation for applications in a similar setting. Due to ongoing research, the identity of these compounds remain confidential.

Compound NTR1 demonstrated few effects against the immune system (Figure 6.7). Activated, inflammatory macrophages only had decreased TNF $\alpha$ production with treatment. Whilst this could be interpreted as an anti-inflammatory effect, during the shorter phagocytosis assay the three measured activities were enhanced, including TNF $\alpha$ production. No effects were seen on cell motility. IFN $\gamma$ from CTL was enhanced whilst this cytokine was suppressed during the helper $\mathrm{T}$ cell activation assay, however a higher concentration of NTR1 was required. B cells were also altered by this compound with increased IL-6 production at low concentrations. B cell viability was reduced with the middle concentrations tested.

NTR2 exhibited different effects on the immune system compared to NTR1 (Figure 6.8). In activated $\mathrm{T}$ cells, pro-inflammatory mediators, such as IFN $\gamma$ and IL-17A, increased whilst anti-inflammatory IL-10 decreased. IFN $\gamma$ production was also enhanced during CTL degranulation. Higher concentrations of NTR2 had suppressive effects across several immune cell processes, whilst lower concentrations appeared to promote anti-inflammatory humoral immunity by enhancing B cell activities and IL-4 production by T cells. It is difficult to decipher the overall effects of this compound on inflammation as both pro-and anti-inflammatory cytokines are simultaneously enhanced and suppressed.

NTR3 also has variable effects on the immune system which differ from the two previous compounds (Figure 6.9). Macrophages were generally unaffected, though some changes to cytokine production were seen, especially at low concentrations. Cell motility was 
enhanced with high concentrations of NTR3 but decreased with a lower concentration. Generally, the effects on lymphocytes are associated with anti-inflammatory effects. T cell production of IL-10 increased while IFN $\gamma$ and IL-17A production decreased. B cells had increased IgM production, alongside enhanced IL-10. In contrast to this anti-inflammatory activity, IFN $\gamma$ production from CTL was enhanced. The three compounds all alter immune cell activities to different extents, and highlight the different effects that are caused by different compound activities. Further investigation is underway at VUW to further understand the immunomodulatory activity of these compounds.
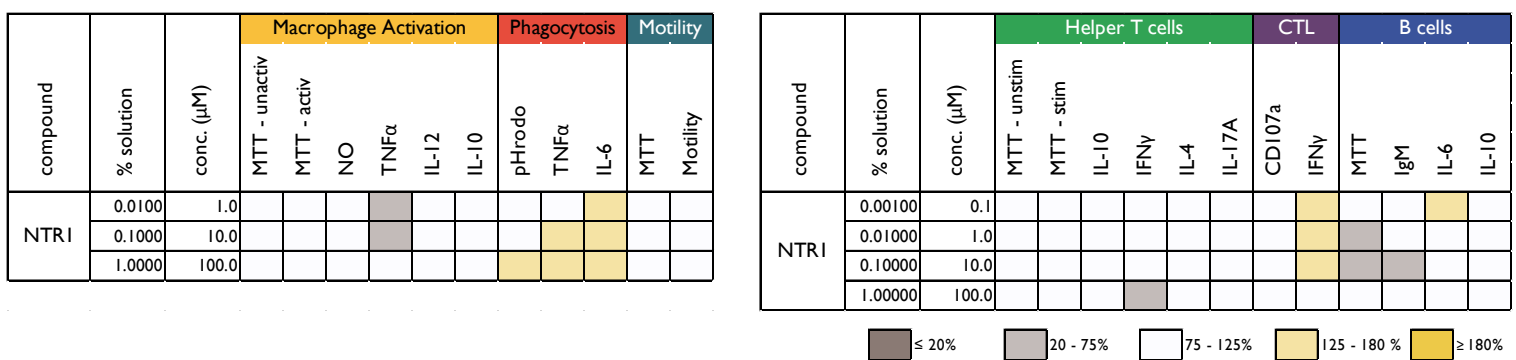

Figure 6.7. Activity profile of NTR1

Categories are based on output levels compared to vehicle controls. Data are from each assay conducted once, in duplicate. Raw data can be found in appendix section 9.6.5.
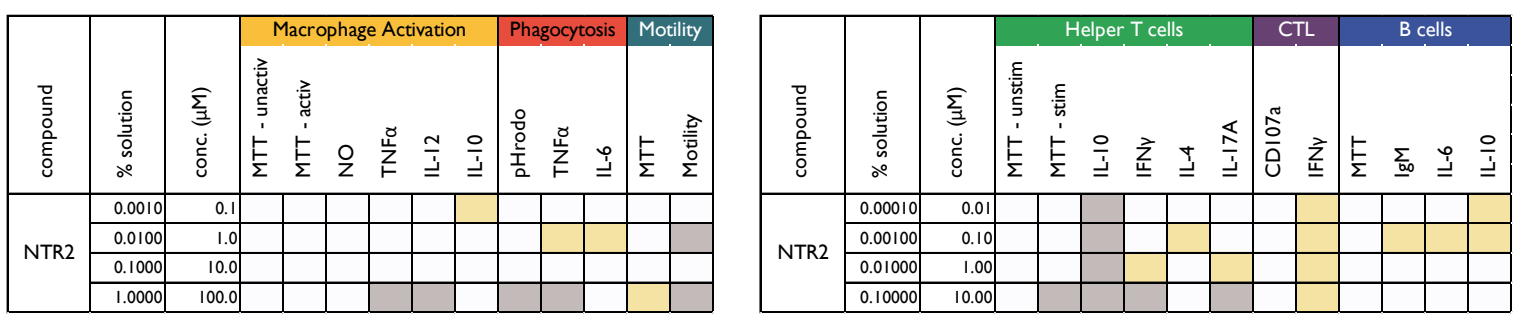

Figure 6.8. Activity profile of NTR2

Categories are based on output levels compared to vehicle controls. Data are from each assay conducted once, in duplicate. Raw data can be found in appendix section 9.6.6.
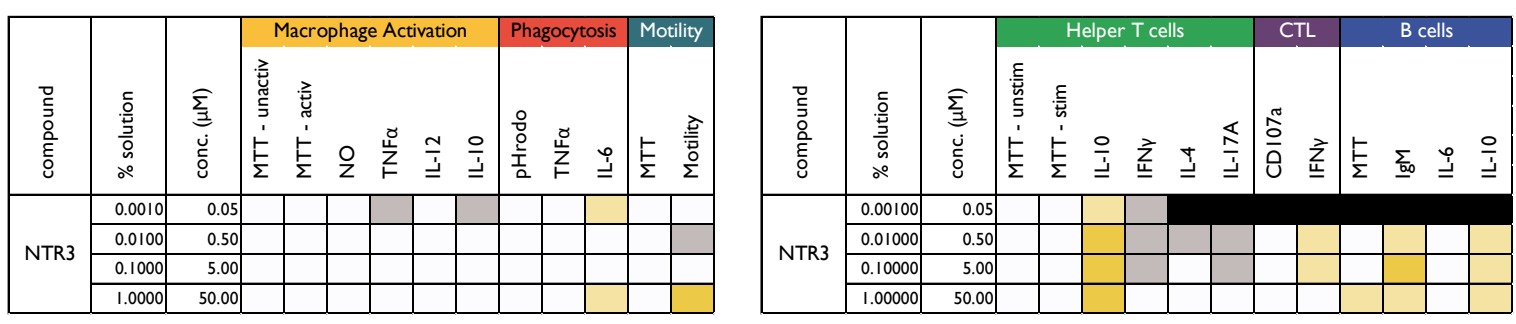

Figure 6.9. Activity profile of NTR3

Categories are based on output levels compared to vehicle controls. Data are from each assay conducted once, in duplicate. Raw data can be found in appendix section 9.6.7. 


\subsubsection{The bioactivity of Peloruside A}

Peloruside A (PelA), a microtubule (MT) stabiliser, was available in limited supply thus was only run through the first stage of the screening strategy[207]. This compound is known to inhibit proliferation, and this was demonstrated by the dividing RAW 264.7 cells succumbing to the compound's effects. Non-proliferative, activated macrophages retained high levels of viability across all concentrations of the compound (Figure 6.10, A). PelA altered cytokine production when RAW 264.7 macrophages were activated, which warrants further investigation using BMDM (Figure 6.10, B). Stimulated Th cells had decreased viability compared to both the vehicle-treated controls and unstimulated counterparts (Figure 6.10, C) whilst T cell cytokine production showed little change with PelA treatment at concentrations where MTT is unaltered (Figure 6.10, D). Although PelA's effects on the immune system may be able to be inferred from other MT stabilisers, its effects on macrophage cytokine production appear unique which may indicate the importance of the different mechanisms between MT stabilisers and their effects on the immune system.

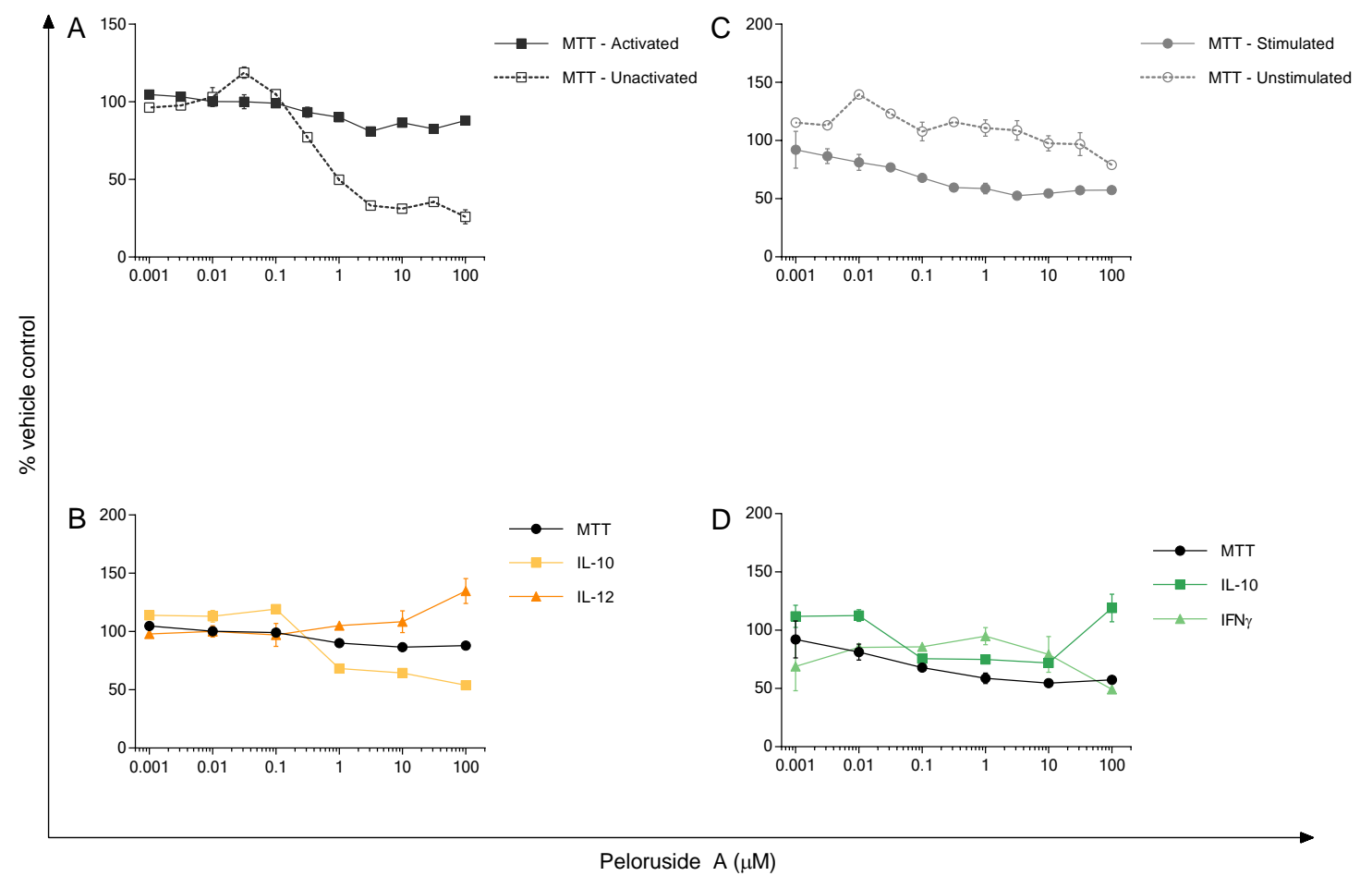

Figure 6.10. PelA alters macrophage cytokine production, and reduces viability of active $T$ cells PelA was screened through the macrophage activation and helper $\mathrm{T}$ cell activation assays at the indicated concentrations. Methodologies follow what is previously described in section 5.3.1. Data are means and SEM, each from one experiment conducted in duplicate. 


\subsubsection{The bioactivity of Hamigeran G}

Hamigeran $\mathrm{G}$ was another novel compound that was under investigation by the Natural Products group at VUW. This compound was extracted from the sea sponge Hamigera tarangaensis and was investigated for any bioactivity as the compound is structurally unique[208]. Hamigeran $G$ was only available in very limited quantities, so it was only run through the macrophage activation assay. This compound exhibited bioactivity, being cytotoxic to both activated and unactivated RAW 264.7 cells (Figure 6.11, A). Hamigeran $\mathrm{G}$ also altered cytokine production in activated macrophages (Figure 6.11, B). The mechanism through which Hamigeran $\mathrm{G}$ acts is unknown, although a link to Golgi function and vesicle transport has been proposed[208].
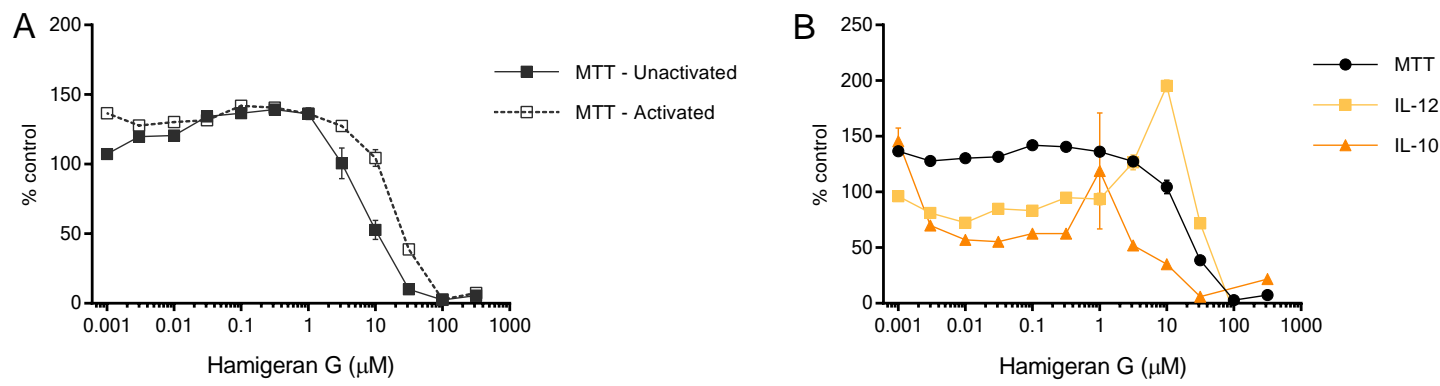

Figure 6.11. Hamigeran $\mathrm{G}$ demonstrates activity against macrophages.

50,000 RAW 264.7 cells were plated and were primed with $20 \mathrm{U} / \mathrm{mL}$ IFN $\gamma$ or remained unprimed. Hamigeran $\mathrm{G}$, or vehicle controls, was added to wells at the indicated concentrations, alongside $200 \mathrm{ng} / \mathrm{mL}$ LPS for stimulation, or remained unstimulated. After $24 \mathrm{~h}$, cell viability was assessed by MTT (A) and supernatants from activated macrophages were analysed for IL-10 and IL-12 production (B). Data are means and SEM from one experiment conducted in duplicate. 


\subsubsection{A cluster analysis for novel compound activity overview}

In the previous chapter, identifying Ixabepilone was based, in part, by having similar immunomodulatory activity to paclitaxel. Because of this, the hypothesis was made that compounds that share cellular targets would have similar effects on immune cell activities. A hierarchical cluster of the activity profile data was generated to determine if this is true. Numbers 1-5 were assigned to the activity profile categories where " 1 " denoted large decrease through to " 5 " which represented a large increase. One or two concentrations of each compound were selected for cluster analysis, chosen for demonstrating the compound's most interesting effects. As testing concentrations were not necessarily the same between macrophage and splenocyte assays, these were designated as 'hi' (high) and 'lo' (low) when two concentrations were selected. Using Ward's method, where clusters are formed based on minimum variance, the dendrogram grouped compounds together based on shared activities across the immune system (Figure 6.12)[209].

Ixabepilone grouped with paclitaxel and colchicine, the other microtubule-targeting compounds. This group is flanked by cycloheximide, ibrutinib and latrunculin A. Based on the activity profiles, these likely cluster together due to their widespread suppressive effects across several immune processes. The following group of ten compounds are likely associated due to their moderately suppressive effects against several immune cell activities. Risperidone and dopamine act through the same system and clustered together due to overlapping anti-inflammatory activities, and similar activity was seen with the two associated plant extracts. The compounds in the next group generally had suppressive effects against one cell type (splenocyte or macrophage), and few effects on the other. Rapamycin and clozapine moderately decreased many splenocyte activities whilst the next group - doxorubicin, high gliotoxin and high brefeldin A - had suppressive effects against macrophage activities.

The lower half of the dendrogram appeared to be made of compounds that have few effects on the immune system, that is, most of their activity profiles show 'no change' to immune cell processes. Ibuprofen suppressed T cell cytokines, altered macrophage cytokines, and enhanced motility, which likely set it apart. Sulpiride had few effects on the immune system, showing suppression of T cell cytokines and increased activities during the shorter phagocytosis and degranulation assays. This activity is shared with NTR1, MTDIA and HS16-38. Glatiramer Acetate similarly only affected the shorter assays or increased some 
T cell cytokines, and NTR2 and the lower BPS concentration shares this activity. The remaining compounds cluster together due to the few effects these compounds exhibited.

The hypothesis is shown to be untrue. Compounds clustered together by having shared activities, and this is not necessarily dependent on having shared mechanisms of action. Although the microtubule-targeting compounds did group together, the opioid-targeting compounds did not. Neither did Risperidone, Dopamine, Clozapine and Sulpiride: the compounds that target the dopamine system. The dendrogram instead highlights differences between compounds which share targets, but have different effects on immune cells. This may be due to specific differences in mechanisms or targets.

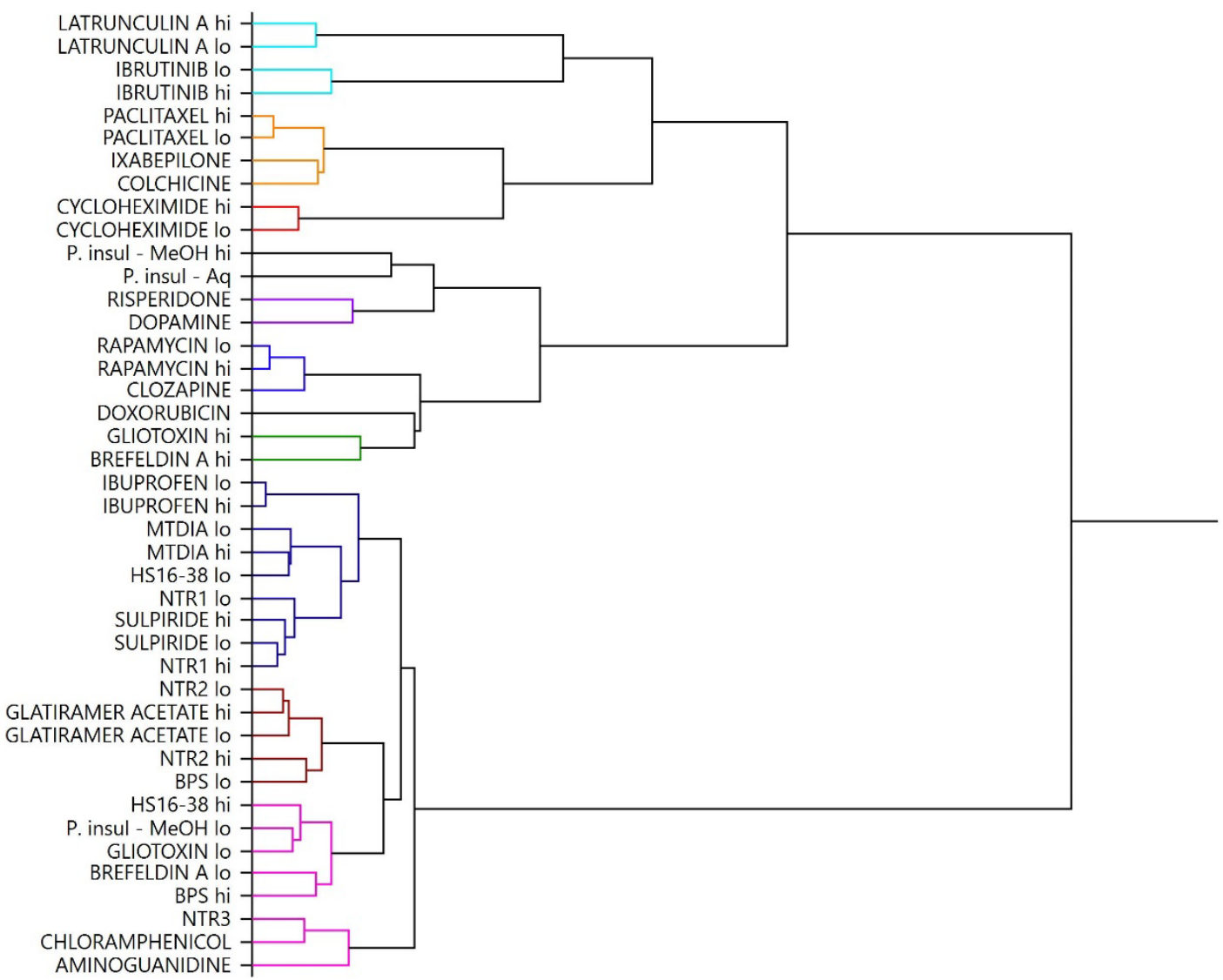

Figure 6.12. A hierarchical cluster shows compounds with shared activities

Using Ward's method, activity profile data from this thesis underwent hierarchical cluster analysis. Each level of change seen in activity profiles were assigned numbers and then clustered using NCSS 12 software. Distances were set to 2 to generate the clusters which are identified by different colours. 


\subsection{Discussion}

The screening strategy provided new insights into the immunomodulatory activities of several novel compounds. P. insularum shows great potential as a source of immunomodulatory compounds, altering many components of the immune system to promote an anti-inflammatory state. As research into MTDIA's anti-cancer activity continues, there is an appreciation of the immunosuppressive side effects that this compound may have. The heparan sulfate mimetic HS16-38 shows potential as an immunomodulator which warrants a deeper investigation for its effect on cell motility. Lastly, NTR3 appears to be a potent anti-inflammatory agent, acting on all of the tested immune cells.

Peloruside A and Hamigeran G were only partially screened due to their limited availability. Peloruside A demonstrated its anti-proliferative effects on the macrophage cell line, and its additional effects on macrophage cytokine production is interesting as it induced different changes compared to Paclitaxel and Ixabepilone, the other MT stabilisers. Hamigeran $\mathrm{G}$ also altered macrophage cytokine production, and the data presented here led to another research project at VUW to further investigate this compound. The ability to generate these data with limited amounts of novel compound highlights the benefit of placing the macrophage and helper $\mathrm{T}$ cell assays first in the screening process. They assess proliferation, cytokine production and, in turn, inflammation, which can inform a decision on which novel compounds are investigated further, and which applications they may suit.

A dendrogram was created from all the screening data as an investigation into an additional way these data could be used. Activity profile data were used to generate this hierarchical cluster analysis; therefore, compounds were associated based on shared effects on immune cell function. Observations from the activity profile data suggested that compounds that share mechanisms of action would have similar bioactivities and cluster together, and this would then generate a hypothesis about a novel compound's target. Though this can happen, e.g. microtubule targeting compounds clustered together, other clusters were formed by compounds that do not share mechanisms (e.g. Brefeldin A and BPS). With further thought, this is not surprising. Screening these compounds looks for changes to cell function, and there are many ways to induce these changes. For example, cytokines can be suppressed by interfering with signalling pathways, or by inhibiting the export of the 
protein through cytoskeleton disruption. After all, this is the reason for phenotypic screening - that many altered mechanisms can induce the same change.

Despite this mistaken presumption, this analysis was useful for identifying the differences between compounds. For example, though the microtubule-targeting compounds cluster together, Ixabepilone is more similar to Colchicine (a stabiliser and destabiliser, respectively), than Paclitaxel, another stabiliser. Similarly, the opioid-targeting compounds did not cluster together. Compounds NTR1, NTR2 and NTR3 work on the same system, but target different opioid receptor subtypes with different affinities which resulted in unique immunomodulatory activities. The dendrogram summarises the data and offers a starting point to generate questions. For example, the dendrogram may spark an interest for the roles of the opioid receptor subtypes in shaping immune responses.

Generating the cluster diagram highlighted the potential to improve data interpretation. Specifically, that more data can refine the way that compound-induced changes to immune cell activities are identified as being moderate, or stronger changes. Currently, a relatively arbitrary threshold is set between each category of change. Thresholds were set to have data fall somewhat evenly between no change, moderate, and large categories of change, but the thresholds may also need to move as additional compounds are screened. To give an example, currently, a compound increasing cell motility to be $150 \%$ of vehicle control is deemed a moderate increase, whereas another compound that increases motility to $180 \%$ is a large increase. However, an additional compound may increase cell motility to $500 \%$. In this case, it would be more appropriate to classify the two previous compounds in the same category as there is an appreciation for how intensely an immune activity could be altered and the first two compounds alter motility to a relatively similar extent. Understanding the possible range of compound-induced changes would be informative and help establish thresholds for classifying the magnitude of activity changes observed due to compounds. With this additional information, thresholds of change for each assay output could be set separately for each particular cell activity as not every output may be able to be increased by the same extent. For example, motility may be able to change five-fold whilst IL-10 from B cells may only double. Although the complete abolition of several outputs has been observed, the full extent of activity increase may not have yet been elicited, and therefore it is hard to categorise the magnitude of observed increases thus far. 


\subsection{Summary}

To summarise, the novel immunomodulatory activity of several compounds were described in this chapter. The cluster analysis identifies compounds that share activities and may prove useful when selecting compounds that are relevant for particular applications, or understanding the differences between compounds that share similar targets but act through different mechanisms. 


\section{Chapter 7: \\ General Discussion}




\subsection{Project Summary}

To facilitate the early identification of potentially immunotoxic and immunomodulatory compounds during drug discovery, a screening strategy was designed through which compounds could be tested in a medium-throughput manner to select candidate compounds for further investigation.

Six cell-based in vitro assays that model key processes implicated in both normal and dysregulated immune function were optimised to be sensitive to any bi-directional changes induced by the compounds. The assays detect changes to immune processes, including macrophage activation, phagocytosis, cell motility, helper T cell activation, B cell activity and CTL degranulation. One set of compounds was run through the six assays to look for any redundancies. That is, where two assays deliver equivalent results due to sharing the same underlying immune cell pathways. Although it was concluded that no such connections existed, the intention was to make the assay system as efficient as possible whereby maximising the information gained while reducing the amount of work required. To this aim, however, it was determined that non-toxic testing concentrations for each compound could be identified by two assays, each representing an immune cell population, and these concentrations could be used throughout the remaining assays. The screening strategy was then optimised for drug discovery. A process was designed to determine the appropriate compound concentrations for testing. This process enables a more efficient use of compounds, and for screening to commence without prior knowledge about compound potency. The assays were then arranged in a cost-effective manner, which centred around the optimal use of animals (the source of primary cells) and reducing the resources required to complete the screen. After confirming that the strategy accurately identified the activity of known immunomodulators, several novel compounds were screened. Many of the compounds had effects on immune cells, some potentially harmful whilst several could offer therapeutic benefit, especially during inflammation.

The resulting screening strategy, which identifies novel immunomodulators, can be used early in drug discovery for many purposes. It can identify potentially immunotoxic compounds during counter-screening stages, or provide insights into novel compound bioactivity. It also facilitates the search for novel immunomodulators to correct immune dysfunction in a medium-throughput format. 


\subsection{About assay design and build}

\subsubsection{General discussion}

This project fulfils the aim of developing a screening platform through which compounds could be assessed for their ability to alter the immune system. Ultimately, a platform was designed that efficiently tests compounds through six assays for immunomodulation with each having multiple outputs and, together, represent a wide range of immune cell activities. A major challenge of the work was to ensure that the assays covered a sufficiently broad spectrum of important immune system function so that compounds did not pass through the assay set as inactive. Though some of the assays have well-established protocols, these required optimisation to meet the needs of the screening strategy. In the end, medium-throughput assays for detecting compound-induced changes were established and performed well when tested with positive controls. Minimising the complexity of assay protocols was a major consideration during assay optimisation, and an example of this is seen with the degranulation assay. This re-designed assay eliminates the need for a twostep culture procedure to test CTL activity. This new method simplifies the assay protocol and reduces its cost. In other cases, existing assay protocols were adjusted to see if more outputs could be generated. For example, the longer duration of the phagocytosis assay allowed for the additional assessment of cytokine production without altering the cost of this assay. The technologies required to run these experiments are, hopefully, available in many laboratories. The assay designs result in an accessible screening platform that models a broad range of immune cell activities.

Though many cell processes are represented in this screening strategy, not all immune cell processes were included. Chemotaxis is not specifically assessed in this screening strategy despite this activity being a successful target for therapy. Granulocytes are only represented by the cellular processes through which they act, e.g. degranulation, though there is an assumption that CTL degranulation adequately represents this function in all degranulating cells. As is inevitable when setting up a broad screening strategy, all variants of a process cannot reasonably be captured. For example, many of the cellular mechanisms for exocytosis are common between cells, however there are some differences. The literature suggests that the loss of Syntaxin-11 - a snare protein - causes the loss of degranulatory ability in CTL and NK cells, but not mast cells[87]. Therefore, while there is a good representation of immune cell activities in the screening strategy, it does not represent all 
possible targets. Along a similar vein, not all potential outputs (e.g. cytokines) from the assays are measured in this screening strategy. More data could be captured using CBA or Bio-Plex assay kits if desired. These assays can measure 30+ cytokines and chemokines from each sample, which can provide data that is more informative for identifying novel therapies and offer deeper insight into compound activity. Despite the wide scope of this screening platform, it is finite. The impact of this limitation will differ depending on the user's needs.

A tactical choice was made to develop a medium-throughput screening strategy. This choice was based on the assumption that the screen would not be used at the very earliest stages of drug discovery, such as during compound library screening, but slightly later when the researcher is interested in rich data on tens to hundreds of compounds. Nonetheless, some uses of this approach would benefit from higher throughput to better suit library screening. Changes could be made to achieve this, for example through the use of 384-well plates. It is important to consider seeding densities, treatment concentrations, and the number of cells needed to generate measurable activity during this exercise.

Using cell lines is potentially another way to improve throughput. However, in this project the use of the RAW 264.7 cell line was problematic in that while the cell line performed as expected in most activities, some specific outputs consistently diverged from reported patterns. Despite this difference, the cell line was able to reproducibly detect change, which was the key purpose. Therefore, cell lines have the potential to replace the primary cells used in this project but only after validating their ability to represent immune cell functions. Cell lines are easier to expand in numbers, which is appealing when screening a large number of compounds and would suit research groups that are interested in reducing the use of animals in research. Human cell lines may produce more 'translatable' data, and could also be explored for use in this screening strategy.

\subsubsection{Future directions}

Assembling the workflow, by which a compound is tested through the assays, was initially an exercise in searching for patterns within the generated data set to identify any assays that were not required. That is, did any of the assay outputs consistently change together 
with various compound treatments to suggest that these processes are mechanistically linked, and therefore measuring both outputs would not be necessary at this stage of compound investigation. This was done by assessing raw data for similar changes to cell activities in a pairwise manner (e.g. phagocytosis and motility). No relationships between outputs were found, however, the possibility that a change to one activity could be implied from a combination of other assay outputs was not explored. For example, if phagocytosis and cell motility both decrease, can a decrease in CTL degranulation be safely assumed? This kind of analysis requires the capacity to handle large data sets, and a process to identify patterns within the data. While developing an analytical strategy for the large data sets generated by this screening strategy was beyond the scope of this project, this work identifies the importance of data management and maximising the use of the available data. This project laid some foundations for collating assay data, through activity profiles, however further development can enhance data collection, storage, and analysis. New findings may arise from these data sets through mining for additional insights on immune cell biology and compound activity.

After establishing this screening strategy and demonstrating its ability to identify immunomodulatory activity, the next step is to ensure the findings are robust. The assays need to perform consistently during each iteration to be a reliable screening platform. Therefore, evaluating assay reproducibility is a high priority. After generating the data presented throughout this thesis, some of the compounds used in Chapter 4 (i.e. screenbuilding set) were screened again. Whilst repeat experiments showed the same direction of change in activity (increase or decrease), the magnitude of change was not always the same (data not shown). Assessing reproducibility with several rounds of repetitious screening of positive controls would identify the range of expected variation and may reveal ways to reduce it. Once understood, a system could be established to allow data to be normalised across rounds of screening based on control compound activity. This normalisation is important for comparing the activities between compounds that were screened separately and is especially important if cluster analyses are used. Because of the variability between runs of experiments during this project, and the outstanding need to test for assay reproducibility, the data presented in this thesis come from single runs of experiments (i.e. $n=1$ ). No statistical analyses of the findings were presented for this reason. Once this variability has been addressed, and repeat experiments have been completed, compound activity can be analysed for statistical significance. 
Z’ factors could be used to evaluate assay quality or ability[210]. This factor takes the spread (standard deviation, SD) of positive control and vehicle data, and divides this by the difference between mean data from these two conditions. If there is a large difference between the two treatment condition means, or if the data from the two conditions have little overlap, this value is small; this number is then taken away from 1 . The ideal Z' factor is close to 1 as this indicates that the assay performs well: that positive control and vehicletreated data are distinct. Not only can a Z' factor evaluate assay sensitivity, for each run of the assay, it can also be used as a robust quality check to ensure the assay has performed well, i.e. has a comparable Z' factor to other runs. This may be a suitable quality control measure for assay performance and reproducibility. This is a statistic commonly used during assay development and screening; it is not the same as the z-score which calculates how many standard deviations a data point is to its population mean.

$$
Z^{\prime} \text { factor }=1-\frac{3\left(S D_{\text {pos ctrl }}+S D_{\text {vehicle }}\right)}{\mid \text { mean } n_{\text {pos ctrl }}-\text { mean } \text { vehicle } \mid}
$$

Other quality controls need to be established for best practice. Compounds can interfere with assays producing false results. For example, some compounds can reduce MTT directly, independent of cells[211,212]. To check this, cell-free treated wells can be run through the MTT assay to assess inherent MTT-reducing activity of compounds. Other interference may come from compounds that are fluorescent, as this screening strategy uses fluorescent signals to measure phagocytosis and degranulation by flow cytometry. It would be desirable to include compound-only wells in such assays to determine if any interference by compounds is meaningful; this also applies to colourimetric assays. In this project, compounds that exhibited higher than average MTT absorbances on unstimulated splenocytes were tested for MTT interference and MTT data were adjusted to acknowledge this additional signal. Where interference is suspected, alternative measures of cell viability or metabolic function can be explored using, for example, RealTime-Glo ${ }^{\mathrm{TM}} \mathrm{MT}$ Cell Viability Assay kits.

After assessing assay reproducibility and introducing quality control measures, a statistical analysis can be applied to the screening outputs to facilitate the assessment of test compound activity. Calculating the effect size of compound activity would be a suitable method to identify compounds that have a significant effect on immune cell activity. Here, 
the difference between mean vehicle- and compound-treated activity is calculated, and this number is divided by the standard deviation seen within the vehicle-treated cells. The final numerical output is the size of effect which states how many standard deviations the compound-treated cell activity is away from the vehicle-treated mean value[213]. In a normal distribution, $95 \%$ of the data are within two SD of the mean, and $99.7 \%$ of the data are within three $\mathrm{SD}[214]$.

$$
\text { Effect size }=\frac{\text { mean }_{\text {treatment }}-\text { mean }_{\text {vehicle }}}{S D_{\text {vehicle }}}
$$

The effect size of compound activity would indicate the magnitude of change seen when cells are treated with compounds, and not simply a probability that any change is statistically significant which is what a p-value denotes. The effect size calculation could be used to set thresholds for identifying compounds that have an effect within various assays. This thesis used $75 \%$ and $125 \%$ of vehicle control to determine that a compound has activity, however these thresholds could be determined statistically by calculating which \% control value is the equivalent of three SDs from the vehicle-treated mean for each assay output in the screening strategy. Any compounds beyond the threshold could be confidently determined to have activity that elicits a functional change in cells.

Threshold value $=3 \times S D_{\text {vehicle }}+$ mean $_{\text {vehicle }}$

$\%$ control value to surpass threshold $=100 \pm\left(\frac{\text { threshold value }_{\text {mean }} \text { vehicle }}{\text { men }}\right) \times 100$ 


\subsection{About assessing immunomodulatory activities}

\subsubsection{General discussion}

This strategy offers a method by which immunotoxicity can be assessed early in the drug discovery and development pipeline, this is particularly important for novel compounds. These novel compounds may interact with targets important to immune cell functions, causing undesired immunological consequences. Identifying this issue at an early stage can be used to guide lead compound selection or to guide future compound development away from undesirable off-target immunological liabilities. Instead of solely assessing viability in a select few cell populations before testing in vivo, as done with other toxicity assessments, the strategy designed in this thesis offers a method to identify effects against a range of immune cells and their activities. These data can also be used to select more appropriate in vivo assays to verify a compound's immunotoxic effects. TDAR (T cell Dependant Antibody Response) assays can be used to confirm compound-induced suppression of B cells, T cells, and innate immune cell activities (motility, phagocytosis); other assays will need to be used for compounds that are shown to enhance these activities, or affect degranulation, cytokine production, or bactericidal activity.

Another motivator for building this strategy was to provide a screening platform to identify compounds that may be developed into drugs to treat immune-mediated diseases. Immune disorders are caused by different aberrant immune activities, so the 'desirable outcome' will differ depending on the disease. For this reason, the broad-scope screen facilitates the search for identifying compounds that induce changes that may be relevant for the treatment of a range immune diseases. This strategy may become increasingly useful as research into immune-mediated disorders progresses, and our understanding of the contributions of different immune cells to specific disease states deepens, thus revealing new targets for disease treatment. A preferred outcome for this strategy is that compound activity profiles can be paired with various immune-mediated diseases by the specific changes to immune cell function induced by test compounds. Researchers investigating compounds for their suitability to treat immune disorders can interrogate compound libraries with this screening strategy to identify compounds that may rectify dysfunctional immune activities whilst leaving functioning immune processes intact. 
This accessible screening strategy was designed for use in a diverse range of research laboratories. Early drug discovery often starts in the chemistry lab which may have limited access to the equipment, materials and expertise to conduct biological testing. This screening strategy is designed to require few technologies, and relatively little experience with immune cells. The wide-scope nature of the strategy also offers a better chance of capturing activity with the subsequent effect of deepening the understanding of novel compound activity and producing enough preliminary data to generate interest in further developing the compound even if the group's main interest is not centred on treating a particular disease.

\subsubsection{Future directions}

One limitation from screening novel compounds with this strategy is the inability to confidently identify compounds that will also modify immune processes in vivo. In this project, prescribed immunomodulators have been used as compounds to build and test the screen. These drugs have demonstrated effects in vivo, and data from screening these compounds in vitro could be used to set thresholds for the activity profiles. For example, colchicine inhibits cell motility in vivo and, in vitro; wound closure by colchicine-treated cells was only $14 \%$ of what vehicle-treated cells achieved. This effect might be a valid indication of the level of change that needs to be observed in vitro to translate to in vivo effects. Being able to reference the level of change caused by test compounds against exemplary compounds might aid the identification of compounds that are likely to have in vivo activity. Screening several drugs with known in vivo activity through the strategy would shed light on how sound this hypothesis is.

Whilst murine cells are convenient as they are easier to source, there are differences between the immune response and cell biology between mice and humans which may mean the data generated with this screening strategy may not be observed clinically[215]. An example of this difference is the impact of myeloperoxidase (MPO) deficiency between the two species; this enzyme plays a role in microbial defence in the innate immune system. A loss of MPO causes a susceptibility to fungal infection in humans whereas in mice there is some retained ability to destroy this type of pathogen as other anti-fungal mechanisms exist[216,217]. Although assessing MPO activity is not included in this strategy, further research into the conservation of the mechanisms that drive the represented immune cell 
functions between species needs to be evaluated to better understand how adequately murine immune cells, and their modulation by test compounds, resemble human immune cells.

Another example is the different iNOS (inducible nitric oxide synthase) activity between the two species. Murine macrophages readily produce NO via iNOS after stimulation with IFN $\gamma$ and LPS in vitro, however there are reports that this is not seen in human cells stimulated in the same way. Here, iNOS expression is only seen in severe disease states and the mechanism of its activation in these states is unknown[218]. Interestingly, it has been suggested that this difference in iNOS induction is only observed due to the methodology used to derive macrophages from human blood monocytes and therefore is an artefact of current cell culturing methods, rather than a biological difference between species[219]. Together, these two examples not only highlight the differences between mice and human immune cells, but also the complexity in assessing translatability: the solution requires more than simply replacing murine cells with human cells in this screening strategy. It appears that there will always be limitations of the various models used within drug discovery. To progress drug development, and this research area, it is prudent to understand what the limitations of the methods are, and to consider these limitations when making conclusions and further decisions during drug discovery and development.

This screening strategy can reveal many immunomodulatory activities of compounds. As with any compound testing, the results seen using in vitro systems need to be validated using in vivo models of disease. Testing compounds outside of the body can indicate the potential activities of compounds, but these activities may not be reflected when tested within the context of a whole organism. The difference may arise through the simple actions of drug metabolism or elimination. Alternatively, there may be compensatory activity within the intact organism where signals from other cells override any compoundinduced changes, or there may be a sequence of detrimental effects that result in a significantly altered immune system despite prior indications of relative safety. Screening data identifies the cellular activities that are affected by compounds and can guide in vivo assay selection to address these concerns. As identified in chapter 6, several novel compounds demonstrated anti-inflammatory activity during the macrophage activation and helper $\mathrm{T}$ cell activation assays. This activity could be tested using in vivo models of 
inflammation, including the paw oedema test. Here, research animals (mice, rats) are treated with compounds or vehicle equivalent, and carrageenan is injected into the paw to induce swelling (oedema). Any reduction of the resulting swelling by treatments can confirm anti-inflammatory activity. This would be useful to identify if the antiinflammatory effects of $P$. insularum seen during screening is effective in whole organisms and is safe for further testing. Compounds seen to inhibit motility or induce an antiinflammatory state would be good candidates as compounds to treat multiple sclerosis. Here, the mouse model of the disease, EAE (experimental autoimmune encephalomyelitis) can be used to determine compound efficacy within an intact dysfunctional immune system whilst also assessing safety. Because of the current need of mouse models and in vivo testing, the use of murine cells in this screening strategy may offer the best progression for compound activity assessment in these early stages of drug discovery. Again, understanding how the results for these mouse studies translate into human cells and human systems will be key to achieving the goal of identifying future immunomodulatory drugs. As our understanding of the causes of immune-mediated diseases progresses, the transition from in vitro findings to in vivo and clinical testing may become clearer, and the predictions of a compound's clinical success based on in vitro data will strengthen. Similarly, if the translatability of in vitro to clinical data improves, it may become plausible to assess immunotoxicity solely with this screening strategy without needing to assess it in vivo. Replacing murine cells with human cells in the strategy's assays may be beneficial in this scenario. 


\subsection{Conclusion}

The aims of this project were to assemble assays that were optimised to identify immunomodulation by test compounds into a medium-throughput screening platform to identify the immunomodulatory activity of compounds. This could be used to identify novel treatments for immune-mediated diseases, and identify compounds that potentially have immunotoxic activity. This aim was achieved, and this project offers the opportunity to screen compounds under investigation early in the drug discovery pipeline for immunomodulatory effects on many processes of the immune system. Although further developments have been identified relating to reproducibility, and translation to in vivo systems, the early applications of the screening strategy are promising. The full screen reveals the activities of known immunomodulatory compounds. Furthermore, on testing a set of compounds with no previously identified immunomodulatory activity, a number of interesting outcomes were observed. For example, novel anti-inflammatory activities were found in drugs that target neurotransmitters, and anti-inflammatory activity was also found in extracts from a Samoan traditional medicine. The potential immunosuppressive activity of MTDIA was identified and may become a concern if this treatment is used to combat cancer progression. 


\section{References}

Mishra, B. B. and Tiwari, V. K. (2011) Natural products: an evolving role in future drug discovery. Eur. J. Med. Chem. 46, 4769-4807.

2 Haefner, B. (2003) Drugs from the deep: marine natural products as drug candidates. Drug Discov. Today 8, 536-544.

3 Butler, M. S. (2004) The Role of Natural Product Chemistry in Drug Discovery ${ }^{\dagger}$. J. Nat. Prod. 67, 2141-2153.

4 Maini, R. N., Elliott, M. J., Brennan, F. M., Williams, R. O., Chu, C. Q., Paleolog, E., Charles, P. J., Taylor, P. C. and Feldmann, M. (1995) Monoclonal anti-TNF alpha antibody as a probe of pathogenesis and therapy of rheumatoid disease. Immunol. Rev. 144, 195-223.

5 Pushpakom, S., Iorio, F., Eyers, P. A., Escott, K. J., Hopper, S., Wells, A., Doig, A., Guilliams, T., Latimer, J., McNamee, C., et al. (2018) Drug repurposing: progress, challenges and recommendations. Nat. Rev. Drug Discov. 18, 41-58.

6 Reaume, A. G. (2011) Drug repurposing through nonhypothesis driven phenotypic screening. Drug Discov. Today Ther. Strateg. 8, 85-88.

7 Ashburn, T. T. and Thor, K. B. (2004) Drug repositioning: identifying and developing new uses for existing drugs. Nat. Rev. Drug Discov. 3, 673-683.

8 Skljarevski, V., Zhang, S., Iyengar, S., D’Souza, D., Alaka, K., Chappell, A. and Wernicke, J. (2011) Efficacy of Duloxetine in Patients with Chronic Pain Conditions. Curr. Drug Ther. 6, 296-303.

9 Lunn, M. P., Hughes, R. A. and Wiffen, P. J. (2014) Duloxetine for treating painful neuropathy, chronic pain or fibromyalgia. Cochrane Database Syst. Rev.

10 Kotz, J. (2012) Phenotypic screening, take two. SciBX Sci.-Bus. Exch. 5.

11 Moffat, J. G., Vincent, F., Lee, J. A., Eder, J. and Prunotto, M. (2017) Opportunities and challenges in phenotypic drug discovery: an industry perspective. Nat. Rev. Drug Discov. 16, 531-543.

12 Chung, T. D. Y., Terry, D. B. and Smith, L. H. (2004) In Vitro and In Vivo Assessment of ADME and PK Properties During Lead Selection and Lead Optimization - Guidelines, Benchmarks and Rules of Thumb. Assay Guid. Man. (Sittampalam, G. S., Coussens, N. P., Brimacombe, K., Grossman, A., Arkin, M., Auld, D., Austin, C., Baell, J., Bejcek, B., Caaveiro, J. M. M., et al., eds.).

13 (2000) Harmonised Tripartite Guideline: Safety Pharmacology Studies for Human Pharmaceuticals S7A. Int. Conf. Harmon.

14 Stark, C. and Steger-Hartmann, T. (2016) Nonclinical Safety and Toxicology. In New Approaches to Drug Discovery (Nielsch, U., Fuhrmann, U., and Jaroch, S., eds.), pp 261-283, Springer International Publishing, Cham.

15 Bova, M. P., Tam, D., McMahon, G. and Mattson, M. N. (2005) Troglitazone induces a rapid drop of mitochondrial membrane potential in liver HepG2 cells. Toxicol. Lett. 155, 41-50.

16 Furberg, C. D. and Pitt, B. (2001) Withdrawal of cerivastatin from the world market. Curr. Control. Trials Cardiovasc. Med. 2, 205-207.

17 Ong, M. M. K., Latchoumycandane, C. and Boelsterli, U. A. (2007) Troglitazone-Induced Hepatic Necrosis in an Animal Model of Silent Genetic Mitochondrial Abnormalities. Toxicol. Sci. 97, 205213.

18 Schirris, T. J. J., Renkema, G. H., Ritschel, T., Voermans, N. C., Bilos, A., van Engelen, B. G. M., Brandt, U., Koopman, W. J. H., Beyrath, J. D., Rodenburg, R. J., et al. (2015) Statin-Induced Myopathy Is Associated with Mitochondrial Complex III Inhibition. Cell Metab. 22, 399-407.

19 (2005) Harmonised Tripartite Guideline: Immunotoxicity Studies for Human Pharmaceuticals S8. Int. Conf. Harmon.

20 Lebrec, H., Molinier, B., Boverhof, D., Collinge, M., Freebern, W., Henson, K., Mytych, D. T., Ochs, H. D., Wange, R., Yang, Y., et al. (2014) The T-cell-dependent antibody response assay in nonclinical studies of pharmaceuticals and chemicals: Study design, data analysis, interpretation. Regul. Toxicol. Pharmacol. 69, 7-21.

21 Luster, M. I., Portier, C., Pait, D. G., White, K. L., Gennings, C., Munson, A. E. and Rosenthal, G. J. (1992) Risk assessment in immunotoxicology: I. Sensitivity and predictability of immune tests. Fundam. Appl. Toxicol. 18, 200-210. 
Tange, S., Scherer, M. N., Graeb, C., Weiss, T., Justl, M., Frank, E., Andrassy, J., Jauch, K.-W. and Geissler, E. K. (2002) The antineoplastic drug Paclitaxel has immunosuppressive properties that can effectively promote allograft survival in a rat heart transplant model. Transplantation 73, 216-223. Iyer, P., Dirweesh, A. and Zijoo, R. (2017) Hydralazine Induced Lupus Syndrome Presenting with Recurrent Pericardial Effusion and a Negative Antinuclear Antibody. Case Rep. Rheumatol. 2017. Kumar, B., Strouse, J., Swee, M., Lenert, P. and Suneja, M. (2018) Hydralazine-associated vasculitis: Overlapping features of drug-induced lupus and vasculitis. Semin. Arthritis Rheum. 48, 283-287. Olsen, N. (2004) Drug-induced autoimmunity. Best Pract. Res. Clin. Rheumatol. 18, 677-688. Pollard, K. M., Hultman, P. and Kono, D. H. (2010) Toxicology of Autoimmune Diseases. Chem. Res. Toxicol. 23, 455-466.

27 Suntharalingam, G., Ward, S. and Brunner, M. D. (2006) Cytokine Storm in a Phase 1 Trial of the AntiCD28 Monoclonal Antibody TGN1412. N Engl J Med 11.

28 Attarwala, H. (2010) TGN1412: From Discovery to Disaster. J. Young Pharm. JYP 2, 332-336.

29 Descotes, J. (2006) Methods of evaluating immunotoxicity. Expert Opin. Drug Metab. Toxicol. 2, 249259.

30 Zhu, X. and Evans, E. (2016) In Vitro Methods in Immunotoxicity Assessment. In Drug Discovery Toxicology (Will, Y., McDuffie, J. E., Olaharski, A. J., and Jeffy, B. D., eds.), pp 193-200, John Wiley \& Sons, Inc, Hoboken, NJ.

31 Kawabata, T. T. and Evans, E. W. (2012) Development of Immunotoxicity Testing Strategies for Immunomodulatory Drugs. Toxicol. Pathol. 40, 288-293.

32 Stout, R. D., Jiang, C., Matta, B., Tietzel, I., Watkins, S. K. and Suttles, J. (2005) Macrophages Sequentially Change Their Functional Phenotype in Response to Changes in Microenvironmental Influences. J. Immunol. 175, 342-349.

33 Mosser, D. M. and Edwards, J. P. (2008) Exploring the full spectrum of macrophage activation. Nat. Rev. Immunol. 8, 958-969.

34 Fang, F. C. (2004) Antimicrobial reactive oxygen and nitrogen species: concepts and controversies. Nat. Rev. Microbiol. 2, 820-832.

35 Weiss, G. and Schaible, U. E. (2015) Macrophage defense mechanisms against intracellular bacteria. Immunol. Rev. 264, 182-203.

36 Blum, J. S., Wearsch, P. A. and Cresswell, P. (2013) Pathways of Antigen Processing. Annu. Rev. Immunol. 31, 443-473.

37 Nathan, C. (2006) Neutrophils and immunity: challenges and opportunities. Nat. Rev. Immunol. 6, 173-182.

38 Stone, K. D., Prussin, C. and Metcalfe, D. D. (2010) IgE, Mast Cells, Basophils, and Eosinophils. J. Allergy Clin. Immunol. 125, S73-S80.

39 Ljunggren, H.-G. and Kärre, K. (1990) In search of the 'missing self': MHC molecules and NK cell recognition. Immunol. Today 11, 237-244.

40 Campbell, K. S. and Hasegawa, J. (2013) Natural killer cell biology: An update and future directions. J. Allergy Clin. Immunol. 132, 536-544.

41 Mosmann, T. R., Cherwinski, H., Bond, M. W., Giedlin, M. A. and Coffman, R. L. (1986) Two types of murine helper T cell clone. I. Definition according to profiles of lymphokine activities and secreted proteins. J. Immunol. Baltim. Md 1950 136, 2348-2357.

42 Murphy, K. M. and Stockinger, B. (2010) Effector T cell plasticity: flexibility in the face of changing circumstances. Nat. Immunol. 11, 674-680.

43 Stoycheva, D., Deiser, K., Starck, L., Nishanth, G., Schluter, D., Uckert, W. and Schuler, T. (2015) IFN- Regulates CD8+ Memory T Cell Differentiation and Survival in Response to Weak, but Not Strong, TCR Signals. J. Immunol. 194, 553-559.

44 Burger, C. and Vitetta, E. S. (1991) The response of B cells in spleen, Peyer's patches, and lymph nodes to LPS and IL-4. Cell. Immunol. 138, 35-43.

45 Griffin, G. K., Newton, G., Tarrio, M. L., Bu, D., Maganto-Garcia, E., Azcutia, V., Alcaide, P., Grabie, N., Luscinskas, F. W., Croce, K. J., et al. (2012) IL-17 and TNF $\alpha$ Sustain Neutrophil Recruitment During Inflammation Through Synergistic Effects on Endothelial Activation. J. Immunol. Baltim. Md 1950 188, 6287-6299.

46 Korn, T., Bettelli, E., Oukka, M. and Kuchroo, V. K. (2009) IL-17 and Th17 Cells. Annu. Rev. Immunol. 27, 485-517.

47 Acosta-Rodriguez, E. V., Rivino, L., Geginat, J., Jarrossay, D., Gattorno, M., Lanzavecchia, A., Sallusto, F. and Napolitani, G. (2007) Surface phenotype and antigenic specificity of human interleukin 17-producing T helper memory cells. Nat. Immunol. 8, 639-646.

48 Kolls, J. K., McCray Jr, P. B. and Chan, Y. R. (2008) Cytokine-mediated regulation of antimicrobial proteins. Nat. Rev. Immunol. 8, 829-835. 
Schubert, U., Antón, L. C., Gibbs, J., Norbury, C. C., Yewdell, J. W. and Bennink, J. R. (2000) Rapid degradation of a large fraction of newly synthesized proteins by proteasomes. Nature 404, 770-774.

50 Reits, E. A., Vos, J. C., Grommé, M. and Neefjes, J. (2000) The major substrates for TAP in vivo are derived from newly synthesized proteins. Nature 404, 774-778.

51 Zhou, F. (2009) Molecular mechanisms of IFN-gamma to up-regulate MHC class I antigen processing and presentation. Int. Rev. Immunol. 28, 239-260.

52 Griffiths, G. M. (1995) The cell biology of CTL killing. Curr. Opin. Immunol. 7, 343-348.

53 Haspot, F., Li, H. W., Lucas, C. L., Fehr, T., Beyaz, S. and Sykes, M. (2014) Allospecific rejection of MHC class I-deficient bone marrow by CD8 T cells. Am. J. Transplant. Off. J. Am. Soc. Transplant. Am. Soc. Transpl. Surg. 14, 49-58.

54 Ingulli, E. (2010) Mechanism of cellular rejection in transplantation. Pediatr. Nephrol. Berl. Ger. 25, 61-74.

55 Rosser, E. C. and Mauri, C. (2015) Regulatory B Cells: Origin, Phenotype, and Function. Immunity 42, 607-612.

56 Ray, A. and Dittel, B. N. (2017) Mechanisms of Regulatory B cell Function in Autoimmune and Inflammatory Diseases beyond IL-10. J. Clin. Med. 6.

57 Forthal, D. N. (2014) Functions of Antibodies. Microbiol. Spectr. 2, 1-17.

58 Charles A Janeway, J., Travers, P., Walport, M. and Shlomchik, M. J. (2001) B-cell activation by armed helper T cells. Immunobiol. Immune Syst. Health Dis. 5th Ed.

59 Lu, M. and Munford, R. (2016) LPS stimulates IgM production in vivo without help from non-B cells. Innate Immun. 22, 307-315.

60 Shen, P. and Fillatreau, S. (2015) Antibody-independent functions of B cells: a focus on cytokines. Nat. Rev. Immunol. 15, 441-451.

61 Cooper, G. S. and Stroehla, B. C. (2003) The epidemiology of autoimmune diseases. Autoimmun. Rev. 2, 119-125.

62 Gren, S. T. and Grip, O. (2016) Role of Monocytes and Intestinal Macrophages in Crohn’s Disease and Ulcerative Colitis. Inflamm. Bowel Dis. 22, 1992-1998.

63 Li, N. and Shi, R.-H. (2018) Updated review on immune factors in pathogenesis of Crohn's disease. World J. Gastroenterol. 24, 15-22.

64 Catalan-Serra, I., Sandvik, A. K., Bruland, T. and Andreu-Ballester, J. C. (2017) Gammadelta T Cells in Crohn's Disease: A New Player in the Disease Pathogenesis? J. Crohns Colitis 11, 1135-1145.

65 Boden, E. K. and Lord, J. D. (2017) CD4 T Cells in IBD: Crossing the Line? Dig. Dis. Sci. 62, 22082210.

66 Ye, B. and van Langenberg, D. R. (2015) Mesalazine preparations for the treatment of ulcerative colitis: Are all created equal? World J. Gastrointest. Pharmacol. Ther. 6, 137-144.

67 Dalbeth, N., Lauterio, T. J. and Wolfe, H. R. (2014) Mechanism of Action of Colchicine in the Treatment of Gout. Clin. Ther. 36, 1465-1479.

68 Kala, M., Miravalle, A. and Vollmer, T. (2011) Recent insights into the mechanism of action of glatiramer acetate. J. Neuroimmunol. 235, 9-17.

69 Pul, R., Moharregh-Khiabani, D., Škuljec, J., Skripuletz, T., Garde, N., Voß, E. V. and Stangel, M. (2011) Glatiramer Acetate Modulates TNF- $\alpha$ and IL-10 Secretion in Microglia and Promotes Their Phagocytic Activity. J. Neuroimmune Pharmacol. 6, 381-388.

70 Weber, M. S. (2004) Multiple sclerosis: glatiramer acetate inhibits monocyte reactivity in vitro and in vivo. Brain 127, 1370-1378.

71 Pinkse, G. G. M., Tysma, O. H. M., Bergen, C. A. M., Kester, M. G. D., Ossendorp, F., Veelen, P. A. van, Keymeulen, B., Pipeleers, D., Drijfhout, J. W. and Roep, B. O. (2005) Autoreactive CD8 T cells associated with $\beta$ cell destruction in type 1 diabetes. Proc. Natl. Acad. Sci. 102, 18425-18430.

72 Qin, H., Trudeau, J. D., Reid, G. S. D., Lee, I.-F., Dutz, J. P., Santamaria, P., Verchere, C. B. and Tan, R. (2004) Progression of spontaneous autoimmune diabetes is associated with a switch in the killing mechanism used by autoreactive CTL. Int. Immunol. 16, 1657-1662.

73 Echeverri, A. F. and Tobón, G. J. (2013) Autoimmune diabetes mellitus (Type 1A), El Rosario University Press.

74 Boorn, J. G. van den, Konijnenberg, D., Dellemijn, T. A. M., Veen, J. P. W. van der, Bos, J. D., Melief, C. J. M., Vyth-Dreese, F. A. and Luiten, R. M. (2009) Autoimmune Destruction of Skin Melanocytes by Perilesional T Cells from Vitiligo Patients. J. Invest. Dermatol. 129, 2220-2232.

75 Rashighi, M. and Harris, J. E. (2017) Vitiligo pathogenesis and emerging treatments. Dermatol. Clin. 35, 257-265.

76 Choi, J., Kim, S. T. and Craft, J. (2012) The Pathogenesis of Systemic Lupus Erythematosus - An Update. Curr. Opin. Immunol. 24, 651-657.

77 Doiron, L. Types of Antibodies Present in Systemic Lupus Erythematosus Patients. 
78 Bijl, M., Reefman, E., Horst, G., Limburg, P. C. and Kallenberg, C. G. M. (2006) Reduced uptake of apoptotic cells by macrophages in systemic lupus erythematosus: correlates with decreased serum levels of complement. Ann. Rheum. Dis. 65, 57-63.

79 Kazkaz, H. (2004) Anti B cell therapy (rituximab) in the treatment of autoimmune diseases. Curr. Opin. Pharmacol. 4, 398-402.

80 Forconi, F. (2015) Three years of ibrutinib in CLL. Blood 125, 2455-2456.

81 Rosenblum, M. D., Gratz, I. K., Paw, J. S. and Abbas, A. K. (2012) Treating Human Autoimmunity: Current Practice and Future Prospects. Sci. Transl. Med. 4, $125 \mathrm{sr} 1$.

82 Chandrashekara, S. (2012) The treatment strategies of autoimmune disease may need a different approach from conventional protocol: A review. Indian J. Pharmacol. 44, 665-671.

83 Sibilia, J. (2004) Novel concepts and treatments for autoimmune disease: ten focal points. Jt. Bone Spine Rev. Rhum. 71, 511-517.

84 Gennari, A., Ban, M., Braun, A., Casati, S., Corsini, E., Dastych, J., Descotes, J., Hartung, T., HooghePeters, R., House, R., et al. (2005) The Use of In Vitro Systems for Evaluating Immunotoxicity: The Report and Recommendations of an ECVAM Workshop. J. Immunotoxicol. 2, 61-83.

85 Boverhof, D. R., Ladics, G., Luebke, B., Botham, J., Corsini, E., Evans, E., Germolec, D., Holsapple, M., Loveless, S. E., Lu, H., et al. (2014) Approaches and considerations for the assessment of immunotoxicity for environmental chemicals: A workshop summary. Regul. Toxicol. Pharmacol. 68, 96-107.

86 Luo, H. R. and Loison, F. (2008) Constitutive neutrophil apoptosis: Mechanisms and regulation. Am. J. Hematol. 83, 288-295.

87 D’Orlando, O., Zhao, F., Kasper, B., Orinska, Z., Müller, J., Hermans-Borgmeyer, I., Griffiths, G. M., Zur Stadt, U. and Bulfone-Paus, S. (2013) Syntaxin 11 is required for NK and CD8 ${ }^{+}$T-cell cytotoxicity and neutrophil degranulation: Immunomodulation. Eur. J. Immunol. 43, 194-208.

88 Winqvist, I. and Olofsson, T. Mechanisms for eosinophil degranulation; release of the eosinophil cationic protein 8 .

89 Lacy, P. (2006) Mechanisms of Degranulation in Neutrophils. Allergy Asthma Clin. Immunol. 2, 98.

90 Martinez, F. O. and Gordon, S. (2014) The M1 and M2 paradigm of macrophage activation: time for reassessment. F1000prime Rep. 6.

91 Mosser, D. M. (2003) The many faces of macrophage activation. J. Leukoc. Biol. 73, 209-212.

92 Mosser, D. M. and Zhang, X. (2008) Activation of Murine Macrophages. Curr. Protoc. Immunol. Ed. John E Coligan Al CHAPTER, Unit.

93 Kharkrang, M. (2010) Mechanisms Involved in Type II Macrophage Activation and Effector Functions (Master of Biomedical Science).

94 O’Sullivan, D. (2012) Using Experimental Autoimmune Encephalomyelitis to Identify Prospective Treatments for Multiple Sclerosis (Doctor of Philosophy), Doctor of Philosophy, Victoria University of Wellington, Wellington.

95 Byrd-Leifer, C. A., Block, E. F., Takeda, K., Akira, S. and Ding, A. (2001) The role of MyD88 and TLR4 in the LPS-mimetic activity of Taxol. Eur. J. Immunol. 31, 2448-2457.

96 Chen, M.-L., Tsai, T.-C., Wang, L.-K., Lin, Y.-Y., Tsai, Y.-M., Lee, M.-C. and Tsai, F.-M. (2012) Risperidone modulates the cytokine and chemokine release of dendritic cells and induces TNF- $\alpha$ directed cell apoptosis in neutrophils. Int. Immunopharmacol. 12, 197-204.

97 Zareie, P. (2017) Investigating the Mechanism by which the Atypical Antipsychotic Clozapine Reduces Disease in Experimental Autoimmune Encephalomyelitis (Doctor of Philosophy), Victoria University of Wellington.

98 Li, W. (2013) Phagocyte dysfunction, tissue aging and degeneration. Ageing Res. Rev. 12.

99 Cannon, G. J. and Swanson, J. A. (1992) The macrophage capacity for phagocytosis. J. Cell Sci. 101, 907-913.

100 Miksa, M., Komura, H., Wu, R., Shah, K. G. and Wang, P. (2009) A novel method to determine the engulfment of apoptotic cells by macrophages using pHrodo succinimidyl ester. J. Immunol. Methods 342, 71-77.

101 de Oliveira, C. A. and Mantovani, B. (1988) Latrunculin A is a potent inhibitor of phagocytosis by macrophages. Life Sci. 43, 1825-1830.

102 Liang, C.-C., Park, A. Y. and Guan, J.-L. (2007) In vitro scratch assay: a convenient and inexpensive method for analysis of cell migration in vitro. Nat. Protoc. 2, 329-333.

103 Hind, L. E., Dembo, M. and Hammer, D. A. (2015) Macrophage motility is driven by frontal-towing with a force magnitude dependent on substrate stiffness. Integr Biol 7, 447-453.

104 Oliveira, C. A., Kashman, Y. and Mantovani, B. (1996) Effects of latrunculin A on immunological phagocytosis and macrophage spreading-associated changes in the F-actin/G-actin content of the cells. Chem. Biol. Interact. 100, 141-153. 
105 Spector, I., Shochet, N. R., Blasberger, D. and Kashman, Y. (1989) Latrunculins-novel marine macrolides that disrupt microfilament organization and affect cell growth: I. Comparison with cytochalasin D. Cell Motil. Cytoskeleton 13, 127-144.

106 Mills, C. D. and Ley, K. (2014) M1 and M2 Macrophages: The Chicken and the Egg of Immunity. J. Innate Immun. 6, 716-726.

107 Wynn, T. A. and Vannella, K. M. (2016) Macrophages in Tissue Repair, Regeneration, and Fibrosis. Immunity 44, 450-462.

108 Prod'homme, T. and Zamvil, S. S. (2018) The Evolving Mechanisms of Action of Glatiramer Acetate. Cold Spring Harb. Perspect. Med. a029249.

109 Dwyer, J. M. and Johnson, C. (1981) The use of concanavalin A to study the immunoregulation of human T cells. Clin. Exp. Immunol. 46, 237-249.

110 Elgueta, R., Benson, M. J., de Vries, V. C., Wasiuk, A., Guo, Y. and Noelle, R. J. (2009) Molecular mechanism and function of CD40/CD40L engagement in the immune system. Immunol. Rev. 229.

111 Klaus, S. J., Pinchuk, L. M., Ochs, H. D., Law, C. L., Fanslow, W. C., Armitage, R. J. and Clark, E. A. Costimulation through CD28 enhances $\mathrm{T}$ cell-dependent $\mathrm{B}$ cell activation via CD40-CD40L interaction. 11.

112 Arnon, R. and Aharoni, R. (2004) Mechanism of action of glatiramer acetate in multiple sclerosis and its potential for the development of new applications. Proc. Natl. Acad. Sci. 101, 14593-14598.

113 Duda, P. W., Schmied, M. C., Cook, S. L., Krieger, J. I. and Hafler, D. A. (2000) Glatiramer acetate (Copaxone ${ }^{\circledR}$ ) induces degenerate, Th2-polarized immune responses in patients with multiple sclerosis. J. Clin. Invest. 105, 967-976.

114 Comi, G., Amato, M. P., Bertolotto, A., Centonze, D., De Stefano, N., Farina, C., Gallo, P., Ghezzi, A., Grimaldi, L. M., Mancardi, G., et al. (2016) The heritage of glatiramer acetate and its use in multiple sclerosis. Mult. Scler. Demyelinating Disord. 1, 6.

115 Chuluundorj, D. (2013) Type II Activation of Monocytes in Multiple Sclerosis (Doctor of Philosophy), Victoria University of Wellington.

116 Guo, C., Manjili, M. H., Subjeck, J. R., Sarkar, D., Fisher, P. B. and Wang, X.-Y. (2013) Therapeutic Cancer Vaccines: Past, Present and Future. Adv. Cancer Res. 119, 421-475.

117 Betts, M. R. and Koup, R. A. (2004) Detection of T-Cell Degranulation: CD107a and b (Biology, B.M. in C., ed.), pp 497-512, Academic Press.

118 Betts, M. R., Brenchley, J. M., Price, D. A., De Rosa, S. C., Douek, D. C., Roederer, M. and Koup, R. A. (2003) Sensitive and viable identification of antigen-specific CD8+ T cells by a flow cytometric assay for degranulation. J. Immunol. Methods 281, 65-78.

119 Brunner, K. T., Mauel, J., Cerottini, J. C. and Chapuis, B. (1968) Quantitative assay of the lytic action of immune lymphoid cells on 51-Cr-labelled allogeneic target cells in vitro; inhibition by isoantibody and by drugs. Immunology 14, 181-196.

120 Sheehy, M. E., McDermott, A. B., Furlan, S. N., Klenerman, P. and Nixon, D. F. (2001) A novel technique for the fluorometric assessment of $\mathrm{T}$ lymphocyte antigen specific lysis. J. Immunol. Methods 249, 99-110.

121 Clarke, S. Rm., Barnden, M., Kurts, C., Carbone, F. R., Miller, J. F. and Heath, W. R. (2000) Characterization of the ovalbumin-specific TCR transgenic line OT-I: MHC elements for positive and negative selection. Immunol. Cell Biol. 78, 110-117.

122 Denton, A. E., Wesselingh, R., Gras, S., Guillonneau, C., Olson, M. R., Mintern, J. D., Zeng, W., Jackson, D. C., Rossjohn, J., Hodgkin, P. D., et al. (2011) Affinity Thresholds for Naive CD8+ CTL Activation by Peptides and Engineered Influenza A Viruses. J. Immunol. 187, 5733-5744.

123 Eisen, H. N., Hou, X. H., Shen, C., Wang, K., Tanguturi, V. K., Smith, C., Kozyrytska, K., Nambiar, L., McKinley, C. A., Chen, J., et al. (2012) Promiscuous binding of extracellular peptides to cell surface class I MHC protein. Proc. Natl. Acad. Sci. 109, 4580-4585.

124 Brennan, A. J., House, I. G., Oliaro, J., Ramsbottom, K. M., Hagn, M., Yagita, H., Trapani, J. A. and Voskoboinik, I. (2014) A Method for Detecting Intracellular Perforin in Mouse Lymphocytes. J. Immunol. 193, 5744-5750.

125 Jenkins, M. R., Tsun, A., Stinchcombe, J. C. and Griffiths, G. M. (2009) The Strength of T Cell Receptor Signal Controls the Polarization of Cytotoxic Machinery to the Immunological Synapse. Immunity 31, 621-631.

126 Kemp, R. A., Backstrom, B. T. and Ronchese, F. (2005) The phenotype of type 1 and type 2 CD8+ T cells activated in vitro is affected by culture conditions and correlates with effector activity. Immunology 115, 315-324.

127 Cho, J.-H., Kim, H.-O., Kim, K.-S., Yang, D.-H., Surh, C. D. and Sprent, J. (2013) Unique Features of Naive CD8+ T Cell Activation by IL-2. J. Immunol. 191, 5559-5573. 
128 van Stipdonk, M. J. B., Lemmens, E. E. and Schoenberger, S. P. (2001) Naïve CTLs require a single brief period of antigenic stimulation for clonal expansion and differentiation. Nat. Immunol. 2, 423429.

129 Shaulov, A. and Murali-Krishna, K. (2008) CD8 T Cell Expansion and Memory Differentiation Are Facilitated by Simultaneous and Sustained Exposure to Antigenic and Inflammatory Milieu. J. Immunol. 180, 1131-1138.

130 Iritani, B. M., Delrow, J., Grandori, C., Gomez, I., Klacking, M., Carlos, L. S. and Eisenman, R. N. (2002) Modulation of T-lymphocyte development, growth and cell size by the Myc antagonist and transcriptional repressor Mad1. EMBO J. 21, 4820-4830.

131 Pollizzi, K. N., Waickman, A. T., Patel, C. H., Sun, I. H. and Powell, J. D. (2015) Cellular Size as a Means of Tracking mTOR Activity and Cell Fate of CD4+ T Cells upon Antigen Recognition. PLoS ONE 10.

132 Oehen, S. and Brduscha-Riem, K. (1998) Differentiation of naive CTL to effector and memory CTL: correlation of effector function with phenotype and cell division. J. Immunol. Baltim. Md 1950 161, 5338-5346.

133 O’Rourke, A. M., Apgar, J. R., Kane, K. P., Martz, E. and Mescher, M. F. (1991) Cytoskeletal function in CD8-and $\mathrm{T}$ cell receptor-mediated interaction of cytotoxic T lymphocytes with class I protein. J. Exp. Med. 173, 241-249.

134 Giuntoli, R. L., Lu, J., Kobayashi, H., Kennedy, R. and Celis, E. (2002) Direct Costimulation of Tumorreactive CTL by Helper T Cells Potentiate Their Proliferation, Survival, and Effector Function. Clin. Cancer Res. 8, 922-931.

135 Umeshappa, C. S., Xie, Y., Xu, S., Nanjundappa, R. H., Freywald, A., Deng, Y., Ma, H. and Xiang, J. (2013) Th Cells Promote CTL Survival and Memory via Acquired pMHC-I and Endogenous IL-2 and CD40L Signaling and by Modulating Apoptosis-Controlling Pathways. PLOS ONE 8, e64787.

136 Barr, T. A., Brown, S., Ryan, G., Zhao, J. and Gray, D. (2007) TLR-mediated stimulation of APC: Distinct cytokine responses of B cells and dendritic cells. Eur. J. Immunol. 37, 3040-3053.

137 Chen, X. and Jensen, P. E. (2008) The role of B lymphocytes as antigen-presenting cells. Arch. Immunol. Ther. Exp. (Warsz.) 56, 77-83.

138 Van Belle, K., Herman, J., Boon, L., Waer, M., Sprangers, B. and Louat, T. (2016) Comparative In Vitro Immune Stimulation Analysis of Primary Human B Cells and B Cell Lines. J. Immunol. Res. 2016, 1-9.

139 Lund, F. E. (2008) Cytokine-producing B lymphocytes-key regulators of immunity. Curr. Opin. Immunol. 20, 332-338.

140 Yanaba, K., Bouaziz, J.-D., Matsushita, T., Tsubata, T. and Tedder, T. F. (2009) The Development and Function of Regulatory B Cells Expressing IL-10 (B10 cells) Requires Antigen Receptor Diversity and TLR Signals. J. Immunol. Baltim. Md 1950 182, 7459-7472.

141 Chun, J. and Hartung, H.-P. (2010) Mechanism of action of oral fingolimod (FTY720) in multiple sclerosis. Clin. Neuropharmacol. 33, 91-101.

142 Rothman, P., Lutzker, S., Cook, W., Coffman, R. and Alt, F. W. (1988) Mitogen plus interleukin 4 induction of $\mathrm{C}$ epsilon transcripts in B lymphoid cells. J. Exp. Med. 168, 2385-2389.

143 Javeed, A., Ashraf, M., Riaz, A., Ghafoor, A., Afzal, S. and Mukhtar, M. M. (2009) Paclitaxel and immune system. Eur. J. Pharm. Sci. 38, 283-290.

144 Chu, S. W. L., Badar, S., Morris, D. L. and Pourgholami, M. H. (2009) Potent Inhibition of Tubulin Polymerisation and Proliferation of Paclitaxel-resistant 1A9PTX22 Human Ovarian Cancer Cells by Albendazole. Anticancer Res. 29, 3791-3796.

145 Nishida, K., Yamasaki, S., Ito, Y., Kabu, K., Hattori, K., Tezuka, T., Nishizumi, H., Kitamura, D., Goitsuka, R., Geha, R. S., et al. (2005) FceRI-mediated mast cell degranulation requires calciumindependent microtubule-dependent translocation of granules to the plasma membrane. J. Cell Biol. 170, 115-126.

146 Abdelrahman, Y., Fararjeh, M., Abdel-Razeq, W., Mohammad, M. K. and Bustanji, Y. (2014) Assessment of possible immunotoxicity of the antipsychotic drug clozapine: Immunotoxicity of clozapine. J. Pharm. Pharmacol. 66, 378-386.

147 Baliga, B. S., Pronczuk, A. W. and Munro, H. N. (1969) Mechanism of cycloheximide inhibition of protein synthesis in a cell-free system prepared from rat liver. J. Biol. Chem. 244, 4480-4489.

148 Hattori, Y., Akimoto, K., Matsumura, M., Tseng, C.-C., Kasai, K. and Shimoda, S.-I. (1996) Effect of cycloheximide on the expression of LPS-inducible iNOS, IFN- $\beta$, and IRF-1 genes in J774 macrophages. IUBMB Life 40, 889-896.

149 Deschesnes, R. G., Patenaude, A., Rousseau, J. L. C., Fortin, J. S., Ricard, C., Côté, M.-F., Huot, J., C.-Gaudreault, R. and Petitclerc, E. (2007) Microtubule-Destabilizing Agents Induce Focal Adhesion Structure Disorganization and Anoikis in Cancer Cells. J. Pharmacol. Exp. Ther. 320, 853-864. 
150 Dahlgren, C., Norberg, B. and Eriksson, S. (1987) The adherence of polymorphonuclear leucocytes to an albumin-coated glass surface. Effects of therapeutic concentrations of the Catharantus derivatives vincristine, vinblastine and vindesine. Acta Med. Scand. 221, 109-113.

151 Stracke, M. L., Soroush, M., Liotta, L. A. and Schiffmann, E. (1993) Cytoskeletal agents inhibit motility and adherence of human tumor cells. Kidney Int. 43, 151-157.

152 Rusan, N. M., Fagerstrom, C. J., Yvon, A.-M. C. and Wadsworth, P. (2001) Cell Cycle-Dependent Changes in Microtubule Dynamics in Living Cells Expressing Green Fluorescent Protein- $\alpha$ Tubulin. Mol. Biol. Cell 12, 971-980.

153 Forth, S. and Kapoor, T. M. (2017) The mechanics of microtubule networks in cell division. J Cell Biol 216, 1525-1531.

154 Misko, T. P., Moore, W. M., Kasten, T. P., Nickols, G. A., Corbett, J. A., Tilton, R. G., McDaniel, M. L., Williamson, J. R. and Currie, M. G. (1993) Selective inhibition of the inducible nitric oxide synthase by aminoguanidine. Eur. J. Pharmacol. 233, 119-125.

155 Cowart, R. E., Singleton, F. L. and Hind, J. S. (1993) A Comparison of Bathophenanthrolinedisulfonic Acid and Ferrozine as Chelators of Iron(II) in Reduction Reactions. Anal. Biochem. 211, 151-155.

156 Agoro, R., Taleb, M., Quesniaux, V. F. J. and Mura, C. (2018) Cell iron status influences macrophage polarization. PLOS ONE 13, e0196921.

157 Mertens, C., Akam, E. A., Rehwald, C., Brüne, B., Tomat, E. and Jung, M. (2016) Intracellular Iron Chelation Modulates the Macrophage Iron Phenotype with Consequences on Tumor Progression. PLOS ONE (Singh, P. K., ed.) 11, e0166164.

158 Leung, S. (2005) Differential Inhibition of Inducible T Cell Cytokine Secretion by Potent Iron Chelators. J. Biomol. Screen. 10, 157-167.

159 Cherayil, B. J. (2010) Iron and immunity: immunological consequences of iron deficiency and overload. Arch. Immunol. Ther. Exp. (Warsz.) 58, 407-415.

160 Alvarez, C. and Sztul, E. S. (1999) Brefeldin A (BFA) disrupts the organization of the microtubule and the actin cytoskeletons. Eur. J. Cell Biol. 78, 1-14.

161 Bershadsky, A. D. and Futerman, A. H. (1994) Disruption of the Golgi apparatus by brefeldin A blocks cell polarization and inhibits directed cell migration. Proc. Natl. Acad. Sci. 91, 5686-5689.

162 Prydz, K., Hansen, S. H., Sandvig, K. and van Deurs, B. (1992) Effects of brefeldin A on endocytosis, transcytosis and transport to the Golgi complex in polarized MDCK cells. J. Cell Biol. 119, 259-272.

163 McKee, E. E., Ferguson, M., Bentley, A. T. and Marks, T. A. (2006) Inhibition of Mammalian Mitochondrial Protein Synthesis by Oxazolidinones. Antimicrob. Agents Chemother. 50, 2042-2049.

164 Tian, F., Wang, C., Tang, M., Li, J., Cheng, X., Zhang, S., Ji, D., Huang, Y. and Li, H. (2016) The antibiotic chloramphenicol may be an effective new agent for inhibiting the growth of multiple myeloma. Oncotarget 7, 51934-51942.

165 Páez, P. L., Becerra, M. C. and Albesa, I. (2008) Chloramphenicol-Induced Oxidative Stress in Human Neutrophils. Basic Clin. Pharmacol. Toxicol. 103, 349-353.

166 DaMert, G. J. and Sohnle, P. G. (1979) Effect of Chloramphenicol on in vitro Function of Lymphocytes. J. Infect. Dis. 139, 220-224.

167 Besser, M. J., Ganor, Y. and Levite, M. (2005) Dopamine by itself activates either D2, D3 or D1/D5 dopaminergic receptors in normal human T-cells and triggers the selective secretion of either IL-10, TNFalpha or both. J. Neuroimmunol. 169, 161-171.

168 Wu, S.-F., Xu, G., Stanley, D., Huang, J. and Ye, G.-Y. (2015) Dopamine modulates hemocyte phagocytosis via a D1-like receptor in the rice stem borer, Chilo suppressalis. Sci. Rep. 5.

169 Matalka, K. Z., Attallah, L. J., Qinna, N. A. and Alhussainy, T. (2011) Dopamine selectively modulates lipopolysaccharide-induced TNF-alpha, IFN-gamma and IL-10 within mice tissues. Neuro Endocrinol. Lett. 32, 176-186.

170 Hasko, G., Szabo, C., Nemeth, Z. H. and Deitch, E. A. (2002) Dopamine suppresses IL-12 p40 production by lipopolysaccharide-stimulated macrophages via a b-adrenoceptor-mediated mechanism. J. Neuroimmunol. 6.

171 Sookhai, S., Wang, J. H., Winter, D., Power, C., Kirwan, W. and Redmond, H. P. (2000) Dopamine attenuates the chemoattractant effect of interleukin-8: a novel role in the systemic inflammatory response syndrome. Shock Augusta Ga 14, 295-299.

172 Denard, B., Lee, C. and Ye, J. (2012) Doxorubicin blocks proliferation of cancer cells through proteolytic activation of CREB3L1. eLife 1.

173 Hussner, J., Ameling, S., Hammer, E., Herzog, S., Steil, L., Schwebe, M., Niessen, J., Schroeder, H. W. S., Kroemer, H. K., Ritter, C. A., et al. (2012) Regulation of Interferon-Inducible Proteins by Doxorubicin via Interferon $\gamma$-Janus Tyrosine Kinase-Signal Transducer and Activator of Transcription Signaling in Tumor Cells. Mol. Pharmacol. 81, 679-688. 
174 Nouri, M. A., Al-Halbosiy, M. M. F., Dheeb, B. I. and Hashim, A. J. (2015) Cytotoxicity and genotoxicity of gliotoxin on human lymphocytes in vitro. J. King Saud Univ. - Sci. 27, 193-197.

175 Kupfahl, C., Geginat, G. and Hof, H. (2006) Gliotoxin-mediated suppression of innate and adaptive immune functions directed against Listeria monocytogenes. Med. Mycol. 44, 591-599.

176 Fiorcari, S., Maffei, R., Audrito, V., Martinelli, S., Hacken, E. ten, Zucchini, P., Grisendi, G., Potenza, L., Luppi, M., Burger, J. A., et al. (2016) Ibrutinib modifies the function of monocyte/macrophage population in chronic lymphocytic leukemia. Oncotarget 7, 65968-65981.

177 Wilson, W. H., Young, R. M., Schmitz, R., Yang, Y., Pittaluga, S., Wright, G., Lih, C.-J., Williams, P. M., Shaffer, A. L., Gerecitano, J., et al. (2015) Targeting B cell receptor signaling with ibrutinib in diffuse large B cell lymphoma. Nat. Med. 21, 922-926.

178 Maddocks, K. and Blum, K. A. (2014) Ibrutinib in B-cell Lymphomas. Curr. Treat. Options Oncol. 15, 226-237.

179 Honigberg, L. A., Smith, A. M., Sirisawad, M., Verner, E., Loury, D., Chang, B., Li, S., Pan, Z., Thamm, D. H., Miller, R. A., et al. (2010) The Bruton tyrosine kinase inhibitor PCI-32765 blocks Bcell activation and is efficacious in models of autoimmune disease and B-cell malignancy. Proc. Natl. Acad. Sci. U. S. A. 107, 13075-13080.

180 Li, D., Rebecca, P., Nurieva, R., Molldrem, J. J., Champlin, R. E. and Ma, Q. (2015) Ibrutinib Treatment Modulates T Cell Activation and Polarization in Immune Response. Blood 126, 3435-3435.

181 Na, Y. R., Yoon, Y. N., Son, D., Jung, D., Gu, G. J. and Seok, S. H. (2015) Consistent Inhibition of Cyclooxygenase Drives Macrophages towards the Inflammatory Phenotype. PLoS ONE 10.

182 Bancos, S., Bernard, M. P., Topham, D. J. and Phipps, R. P. (2009) Ibuprofen and other widely used non-steroidal anti-inflammatory drugs inhibit antibody production in human cells. Cell. Immunol. 258, $18-28$.

183 Hall, V. C. and Wolf, R. E. (1997) Effects of tenidap and nonsteroidal antiinflammatory drugs on the response of cultured human T cells to interleukin 2 in rheumatoid arthritis. J. Rheumatol. 24, 14671470.

184 Paccani, S. R., Boncristiano, M., Ulivieri, C., D’Elios, M. M., Prete, G. D. and Baldari, C. T. (2002) Nonsteroidal Anti-inflammatory Drugs Suppress T-cell Activation by Inhibiting p38 MAPK Induction. J. Biol. Chem. 277, 1509-1513.

185 Osma-Garcia, I. C., Punzón, C., Fresno, M. and Díaz-Muñoz, M. D. (2016) Dose-dependent effects of prostaglandin E2 in macrophage adhesion and migration. Eur. J. Immunol. 46, 677-688.

186 Nakatsugi, S., Sugimoto, N. and Furukawa, M. (1996) Effects of non-steroidal anti-inflammatory drugs on prostaglandin E2 production by cyclooxygenase-2 from endogenous and exogenous arachidonic acid in rat peritoneal macrophages stimulated with lipopolysaccharide. Prostaglandins Leukot. Essent. Fatty Acids 55, 451-457.

187 Lopus, M., Smiyun, G., Miller, H., Oroudjev, E., Wilson, L. and Jordan, M. A. (2015) Mechanism of action of ixabepilone and its interactions with the $\beta$ III-tubulin isotype. Cancer Chemother. Pharmacol. 76, 1013-1024.

188 Lautenschlager, F., Paschke, S., Schinkinger, S., Bruel, A., Beil, M. and Guck, J. (2009) The regulatory role of cell mechanics for migration of differentiating myeloid cells. Proc. Natl. Acad. Sci. 106, 1569615701.

189 Morice, W. G., Brunn, G. J., Wiederrecht, G., Siekierka, J. J. and Abraham, R. T. (1993) Rapamycininduced inhibition of p34cdc2 kinase activation is associated with G1/S-phase growth arrest in T lymphocytes. J. Biol. Chem. 268, 3734-3738.

190 Fox, R., Nhan, T. Q., Law, G. L., Morris, D. R., Liles, W. C. and Schwartz, S. M. (2007) PSGL-1 and mTOR regulate translation of ROCK-1 and physiological functions of macrophages. EMBO J. 26, 505515.

191 Donahue, A. C. and Fruman, D. A. (2007) Distinct signaling mechanisms activate the target of rapamycin in response to different B-cell stimuli. Eur. J. Immunol. 37, 2923-2936.

192 Molimau-Samasoni, S. (2016) Exploring the Molecular Mechanisms of Action of Samoan Medicinal Plants via Chemical Genetic Analyses (Doctor of Philosophy), Victoria University of Wellington.

193 Coorey, N. V. C. (2017) Elucidating the anti-cancer mechanisms for transition-state structure inhibitors of nucleoside phosphorylases methylthioadenosine-DADMe-immucillin A and forodesine. (Doctor of Philosophy), Victoria University of Wellington.

194 Di Padova, F., Di Padova, C., Stramentinoli, G. and Tritapepe, R. (1985) Inhibition of lymphocyte function by a naturally occurring nucleoside: 5'-Methylthioadenosine (MTA). Int. J. Immunopharmacol. 7, 193-198.

195 Cerri, M. A., Beltrán-Nuñez, A., Bernasconi, S., Dejana, E., Bassi, L. and Bazzoni, G. (1993) Inhibition of cytokine production and endothelial expression of adhesion antigens by 5 -methylthioadenosine. Eur. J. Pharmacol. 232, 291-294. 
196 Keyel, P. A., Romero, M., Wu, W., Kwak, D. H., Zhu, Q., Liu, X. and Salter, R. D. (2014) Methylthioadenosine Reprograms Macrophage Activation through Adenosine Receptor Stimulation. PLOS ONE 9.

197 Simon Davis, D. A. and Parish, C. R. (2013) Heparan Sulfate: A Ubiquitous Glycosaminoglycan with Multiple Roles in Immunity. Front. Immunol. 4.

198 Wrenshall, L. E., Stevens, R. B., Cerra, F. B. and Platt, J. L. (1999) Modulation of macrophage and B cell function by glycosaminoglycans. J. Leukoc. Biol. 66, 391-400.

199 Gutter-Kapon, L., Alishekevitz, D., Shaked, Y., Li, J.-P., Aronheim, A., Ilan, N. and Vlodavsky, I. (2016) Heparanase is required for activation and function of macrophages. Proc. Natl. Acad. Sci. 113, E7808-E7817.

200 Veeresham, C. (2012) Natural products derived from plants as a source of drugs. J. Adv. Pharm. Technol. Res. 3, 200-201.

201 Balandrin, M. F., Kinghorn, A. D. and Farnsworth, N. R. (1993) Plant-Derived Natural Products in Drug Discovery and Development: An Overview. In Human Medicinal Agents from Plants (Kinghorn, A. D., and Balandrin, M. F., eds.), pp 2-12, American Chemical Society, Washington, DC.

202 Cox, P. A., Sperry, L. R., Tuominen, M. and Bohlin, L. (1989) Pharmacological activity of the Samoan ethnopharmacopoeia. Econ. Bot. 43, 487-497.

203 Dunstan, C. A., Noreen, Y., Serrano, G., Cox, P. A., Perera, P. and Bohlin, L. (1997) Evaluation of some Samoan and Peruvian medicinal plants by prostaglandin biosynthesis and rat ear oedema assays. J. Ethnopharmacol. 57, 35-56.

204 Basu, I., Locker, J., Cassera, M. B., Belbin, T. J., Merino, E. F., Dong, X., Hemeon, I., Evans, G. B., Guha, C. and Schramm, V. L. (2011) Growth and Metastases of Human Lung Cancer Are Inhibited in Mouse Xenografts by a Transition State Analogue of 5'-Methylthioadenosine Phosphorylase. J. Biol. Chem. 286, 4902-4911.

205 Bistulfi, G., Affronti, H. C., Foster, B. A., Karasik, E., Gillard, B., Morrison, C., Mohler, J., Phillips, J. G. and Smiraglia, D. J. (2016) The essential role of methylthioadenosine phosphorylase in prostate cancer. Oncotarget 7, 14380-14393.

206 Strobl, C. D., Henrich, F., Singer, K., Peter, K., Kreutz, M., Kremer, A., Mackensen, A. and Aigner, M. (2017) MTA-mediated inhibition of human T cells: Mechanism and MTAP overexpression as putative overcoming strategy. Cancer Res. 77, 3971-3971.

207 Hood, K. A., West, L. M., Rouwé, B., Northcote, P. T., Berridge, M. V., Wakefield, S. J. and Miller, J. H. (2002) Peloruside A, a novel antimitotic agent with paclitaxel-like microtubule- stabilizing activity. Cancer Res. 62, 3356-3360.

208 Singh, A. J., Dattelbaum, J. D., Field, J. J., Smart, Z., Woolly, E. F., Barber, J. M., Heathcott, R., Miller, J. H. and Northcote, P. T. (2013) Structurally diverse hamigerans from the New Zealand marine sponge Hamigera tarangaensis: NMR-directed isolation, structure elucidation and antifungal activity. Org. Biomol. Chem. 11, 8041-8051.

209 Ward, J. H. (1963) Hierarchical Grouping to Optimize an Objective Function. J. Am. Stat. Assoc. 58, 236-244.

210 Zhang, J.-H. (1999) A Simple Statistical Parameter for Use in Evaluation and Validation of High Throughput Screening Assays. J. Biomol. Screen. 4, 67-73.

211 Shoemaker, M., Cohen, I. and Campbell, M. (2004) Reduction of MTT by aqueous herbal extracts in the absence of cells. J. Ethnopharmacol. 93, 381-384.

212 Talorete, T. P. N., Bouaziz, M., Sayadi, S. and Isoda, H. (2006) Influence of medium type and serum on MTT reduction by flavonoids in the absence of cells. Cytotechnology 52, 189-198.

213 Ialongo, C. (2016) Understanding the effect size and its measures. Biochem. Medica 150-163.

214 Pukelsheim, F. (1994) The Three Sigma Rule. Am. Stat. 48, 88.

215 Mestas, J. and Hughes, C. C. W. (2004) Of Mice and Not Men: Differences between Mouse and Human Immunology. J. Immunol. 172, 2731-2738.

216 Brennan, M. L., Anderson, M. M., Shih, D. M., Qu, X. D., Wang, X., Mehta, A. C., Lim, L. L., Shi, W., Hazen, S. L., Jacob, J. S., et al. (2001) Increased atherosclerosis in myeloperoxidase-deficient mice. J. Clin. Invest. 107, 419-430.

217 Lehrer, R. I. and Cline, M. J. (1969) Leukocyte myeloperoxidase deficiency and disseminated candidiasis: the role of myeloperoxidase in resistance to Candida infection. J. Clin. Invest. 48, 14781488.

218 Zschaler, J., Schlorke, D. and Arnhold, J. (2014) Differences in innate immune response between man and mouse. Crit. Rev. Immunol.

219 Thomas, A. C. (2014) “Of mice and men”: arginine metabolism in macrophages. Front. Immunol. 7. 
Appendices 


\subsection{Recipes}

\section{Complete T cell Media (CTCM)}

86\% DMEM

$1 \%$ PenStrep $(10,000 \mathrm{U} / \mathrm{mL}$ Penicillin, $10 \mathrm{mg} / \mathrm{mL}$ Streptomycin)

$1 \%$ HEPES (1M)

1\% MEM NEAA (100X, Gibco)

$1 \%$ L-glutamine (200mM)

$0.1 \% \quad 2-\mathrm{Me}(55 \mathrm{mM})$

$10 \% \quad$ FCS

\section{PBS}

$145 \mathrm{mM} \mathrm{NaCl}$

$8.7 \mathrm{mM} \quad \mathrm{Na}_{2} \mathrm{HPO}_{4} .12 \mathrm{H}_{2} \mathrm{O}$

$1.3 \mathrm{mM} \quad \mathrm{NaH}_{2} \mathrm{PO}_{4} .2 \mathrm{H}_{2} \mathrm{O}$

in $\mathrm{ddH}_{2} \mathrm{O}$

\section{FACS Buffer}

$2 \%(v / v) \quad$ FCS

$15 \mathrm{mM} \quad$ Sodium azide

in PBS

\section{MTT Solution}

$5 \mathrm{mg} / \mathrm{mL}$ MTT in PBS

\section{MTT Solubiliser}

$10 \%$ SDS

45\% DMF

in $\mathrm{ddH}_{2} \mathrm{O}, \mathrm{pH} 4.5$ 


\section{ELISA Capture buffer}

$0.1 \mathrm{M} \mathrm{Na} \mathrm{Na}_{2} \mathrm{HPO}_{4}$ in $\mathrm{dd} \mathrm{H}_{2} \mathrm{O}$

pH kept at 9.0, or adjusted to 6.0 with $\mathrm{NaH}_{2} \mathrm{PO}_{4}$

\section{ELISA Stop solution}

$0.18 \mathrm{M} \mathrm{H}_{2} \mathrm{SO}_{4}$ in $\mathrm{ddH}_{2} \mathrm{O}$

\section{ELISA Wash solution}

0.05\% Tween20 in PBS

\section{TMB}

Equal volumes TMB A and TMB B (BD Biosciences, Cat 554214)

\section{Griess Reagent}

equal volumes Griess A and Griess B mixed just prior to use:

Griess A

$1 \%(\mathrm{w} / \mathrm{v}) \quad$ Sulfanilamide

$2.5 \%(\mathrm{v} / \mathrm{v})$ Phosphoric acid

in $\mathrm{ddH}_{2} \mathrm{O}$

Griess B

$0.1 \%(\mathrm{w} / \mathrm{v}) \mathrm{N}$-(1-naphthyl)ethylenediamine

2.5\% (v/v) Phosphoric acid

in $\mathrm{ddH}_{2} \mathrm{O}$

\section{Live Cell Imaging Solution (for pHrodo bioparticles)}

$\begin{array}{ll}140 \mathrm{mM} & \mathrm{NaCl} \\ 2.5 \mathrm{mM} & \mathrm{KCl} \\ 1.8 \mathrm{mM} & \mathrm{CaCl}_{2} \\ 1.0 \mathrm{mM} & \mathrm{MgCl}_{2} \\ 20 \mathrm{mM} & \text { HEPES } \\ \text { in ddH } & \text { O, adjusted } \mathrm{pH} 7.4\end{array}$




\subsection{Reagents}

\subsubsection{Compounds}

Latrunculin A, colchicine, cycloheximide, paclitaxel, gliotoxin, ibuprofen, aminoguanidine, sulpiride, and dopamine were all supplied by Sigma-Aldrich (MO, USA)

Glatiramer acetate was supplied by Sanofi Aventis (France). Brefeldin A and rapamycin were supplied by BioLegend (CA, USA). Doxorubicin was supplied by Calbiochem (Darmstatd, Germany), and ixabepilone was supplied by Bristol-Myers Squibb (NY, USA). Ibrutinib was supplied by Selleck Chemicals (Houston, USA).

Clozapine and Risperidone were kind donations from Douglas Pharmaceuticals (Auckland, NZ). HS16-38 was a kind donation by the Ferrier Institute (Wellington, NZ). Research groups at VUW kindly donated NTR1-3, the P. insularum extracts, BPS, Chloramphenicol, MTDIA, Peloruside A and Hamigeran G.

\begin{tabular}{|l|l|}
\hline Compound & Vehicle \\
\hline Latrunculin A & Ethanol \\
\hline Colchicine & Ethanol \\
\hline Cycloheximide & Ethanol \\
\hline Paclitaxel & Ethanol \\
\hline Risperidone & 0.1 M Acetic acid \\
\hline Clozapine & 0.1 M Acetic acid \\
\hline Brefeldin A & DMSO \\
\hline Aminoguanidine & Water \\
\hline Sulpiride & Ethanol \\
\hline Doxorubicin & PBS \\
\hline Ixabepilone & Ethanol \\
\hline Ibrutinib & DMSO \\
\hline BPS & Water \\
\hline Glatiramer acetate & Water \\
\hline
\end{tabular}

\begin{tabular}{|l|l|}
\hline Compound & Vehicle \\
\hline Dopamine & Ethanol \\
\hline Gliotoxin & DMSO \\
\hline Ibuprofen & Ethanol \\
\hline Rapamycin & Ethanol \\
\hline Chloramphenicol & Ethanol \\
\hline HS16-38 & PBS \\
\hline NTR1 & Water \\
\hline NTR2 & Methanol \\
\hline NTR3 & $1: 1$ DMSO/Methanol \\
\hline P. insularum & Water \\
\hline P. insularum & DMSO \\
\hline MTDIA & DMSO \\
\hline Peloruside A & Ethanol \\
\hline Hamigeran G & DMSO \\
\hline & \\
\hline
\end{tabular}


9.2.2 ELISA reagents

\begin{tabular}{|c|c|c|c|c|c|}
\hline Target & Component & Conc. (in $50 \mu \mathrm{L}$ ) & Stock conc. & Diluent & Supplier \\
\hline $\mathrm{IFN} \gamma$ & $\begin{array}{l}\text { Capture } \\
\text { Top Standard } \\
\text { Detection } \\
\text { SA-HRP }\end{array}$ & $\begin{array}{l}1 \mu \mathrm{g} / \mathrm{mL} \\
4 \mathrm{ng} / \mathrm{mL} \\
125 \mathrm{ng} / \mathrm{mL} \\
1: 2000 \text { dilution }\end{array}$ & $\begin{array}{l}1 \mathrm{mg} / \mathrm{ml} \\
400 \mathrm{ng} / \mathrm{mL} \\
500 \mu \mathrm{g} / \mathrm{mL}\end{array}$ & $\begin{array}{l}0.1 \mathrm{M} \mathrm{Na}_{2} \mathrm{HPO}_{4}, \mathrm{pH} 9.0 \\
5 \% \mathrm{FCS} / \mathrm{PBS} \\
5 \% \mathrm{FCS} / \mathrm{PBS} \\
5 \% \mathrm{FCS} / \mathrm{PBS}\end{array}$ & $\begin{array}{l}\text { BD Pharmingen } \\
\text { BD Pharmingen }\end{array}$ \\
\hline IL-2 & $\begin{array}{l}\text { Capture } \\
\text { Top Standard } \\
\text { Detection } \\
\text { SA-HRP }\end{array}$ & $\begin{array}{l}500 \mathrm{ng} / \mathrm{mL} \\
1 \mathrm{ng} / \mathrm{mL} \\
500 \mathrm{ng} / \mathrm{mL} \\
1: 2000 \text { dilution }\end{array}$ & $\begin{array}{l}500 \mu \mathrm{g} / \mathrm{mL} \\
500 \mathrm{ng} / \mathrm{mL} \\
500 \mu \mathrm{g} / \mathrm{mL}\end{array}$ & $\begin{array}{l}0.1 \mathrm{M} \mathrm{Na}_{2} \mathrm{HPO}_{4}, \mathrm{pH} 9.0 \\
5 \% \mathrm{FCS} / \mathrm{PBS} \\
5 \% \mathrm{FCS} / \mathrm{PBS} \\
5 \% \mathrm{FCS} / \mathrm{PBS}\end{array}$ & $\begin{array}{l}\text { eBiosciences* } \\
\text { eBiosciences* } \\
\text { BD Pharmingen }\end{array}$ \\
\hline IL-4 & $\begin{array}{l}\text { Capture } \\
\text { Top Standard } \\
\text { Detection } \\
\text { SA-HRP }\end{array}$ & $\begin{array}{l}250 \mathrm{ng} / \mathrm{mL} \\
4 \mathrm{ng} / \mathrm{mL} \\
125 \mathrm{ng} / \mathrm{mL} \\
1: 2000 \text { dilution }\end{array}$ & $\begin{array}{l}500 \mu \mathrm{g} / \mathrm{mL} \\
400 \mathrm{ng} / \mathrm{mL} \\
500 \mu \mathrm{g} / \mathrm{mL}\end{array}$ & $\begin{array}{l}\text { PBS } \\
5 \% \text { FCS/PBS } \\
5 \% \text { FCS/PBS } \\
5 \% \text { FCS/PBS }\end{array}$ & $\begin{array}{l}\text { BD Pharmingen } \\
\text { BD Pharmingen } \\
\text { BD Pharmingen }\end{array}$ \\
\hline IL-6 & $\begin{array}{l}\text { Capture } \\
\text { Top Standard } \\
\text { Detection } \\
\text { SA-HRP }\end{array}$ & $\begin{array}{l}1 \mu \mathrm{g} / \mathrm{mL} \\
2 \mathrm{ng} / \mathrm{mL} \\
500 \mathrm{ng} / \mathrm{mL} \\
1: 2000 \text { dilution }\end{array}$ & $\begin{array}{l}1 \mathrm{mg} / \mathrm{mL} \\
200 \mathrm{ng} / \mathrm{mL} \\
500 \mu \mathrm{g} / \mathrm{mL}\end{array}$ & $\begin{array}{l}0.1 \mathrm{M} \mathrm{Na}_{2} \mathrm{HPO}_{4}, \mathrm{pH} 9.0 \\
5 \% \mathrm{FCS} / \mathrm{PBS} \\
5 \% \mathrm{FCS} / \mathrm{PBS} \\
5 \% \mathrm{FCS} / \mathrm{PBS}\end{array}$ & $\begin{array}{l}\text { BD Pharmingen } \\
\text { BD Pharmingen } \\
\text { BD Pharmingen }\end{array}$ \\
\hline IL-10 & $\begin{array}{l}\text { Capture } \\
\text { Top Standard } \\
\text { Detection } \\
\text { SA-HRP }\end{array}$ & $\begin{array}{l}5 \mu \mathrm{g} / \mathrm{mL} \\
10 \mathrm{ng} / \mathrm{mL} \\
200 \mathrm{ng} / \mathrm{mL} \\
1: 1000 \text { dilution }\end{array}$ & $\begin{array}{l}1 \mathrm{mg} / \mathrm{mL} \\
400 \mathrm{ng} / \mathrm{mL} \\
500 \mu \mathrm{g} / \mathrm{mL}\end{array}$ & $\begin{array}{l}0.1 \mathrm{M} \mathrm{Na}_{2} \mathrm{HPO}_{4}, \mathrm{pH} 6.0 \\
5 \% \mathrm{FCS} / \mathrm{PBS} \\
5 \% \mathrm{FCS} / \mathrm{PBS} \\
5 \% \mathrm{FCS} / \mathrm{PBS}\end{array}$ & $\begin{array}{l}\text { BD Pharmingen } \\
\text { BD Pharmingen } \\
\text { BD Pharmingen }\end{array}$ \\
\hline $\begin{array}{c}\text { IL-12 } \\
\text { p40/p70 }\end{array}$ & $\begin{array}{l}\text { Capture } \\
\text { Top Standard } \\
\text { Detection } \\
\text { SA-HRP }\end{array}$ & $\begin{array}{l}1 \mu \mathrm{g} / \mathrm{mL} \\
4 \mathrm{ng} / \mathrm{mL} \\
1 \mu \mathrm{g} / \mathrm{mL} \\
1: 2000 \text { dilution }\end{array}$ & $\begin{array}{l}1 \mathrm{mg} / \mathrm{mL} \\
200 \mathrm{ng} / \mathrm{mL} \\
500 \mu \mathrm{g} / \mathrm{mL}\end{array}$ & $\begin{array}{l}0.1 \mathrm{M} \mathrm{Na}_{2} \mathrm{HPO}_{4}, \mathrm{pH} 9.0 \\
5 \% \mathrm{FCS} / \mathrm{PBS} \\
5 \% \mathrm{FCS} / \mathrm{PBS} \\
5 \% \mathrm{FCS} / \mathrm{PBS}\end{array}$ & $\begin{array}{l}\text { BD Pharmingen } \\
\text { BD Pharmingen } \\
\text { BD Pharmingen }\end{array}$ \\
\hline IL-17A & $\begin{array}{l}\text { Capture } \\
\text { Top Standard } \\
\text { Detection } \\
\text { SA-HRP }\end{array}$ & $\begin{array}{l}1 \mu \mathrm{g} / \mathrm{mL} \\
2 \mathrm{ng} / \mathrm{mL} \\
1 \mu \mathrm{g} / \mathrm{mL} \\
1: 1000 \text { dilution }\end{array}$ & $\begin{array}{l}500 \mu \mathrm{g} / \mathrm{mL} \\
2 \mu \mathrm{g} / \mathrm{mL} \\
500 \mu \mathrm{g} / \mathrm{mL}\end{array}$ & $\begin{array}{l}0.1 \mathrm{M} \mathrm{Na}_{2} \mathrm{HPO}_{4}, \mathrm{pH} 9.0 \\
5 \% \mathrm{FCS} / \mathrm{PBS} \\
5 \% \mathrm{FCS} / \mathrm{PBS} \\
5 \% \mathrm{FCS} / \mathrm{PBS}\end{array}$ & $\begin{array}{l}\text { BD Pharmingen } \\
\text { BD Pharmingen } \\
\text { BD Pharmingen }\end{array}$ \\
\hline $\mathrm{TNF} \alpha$ & $\begin{array}{l}\text { Capture } \\
\text { Top Standard } \\
\text { Detection } \\
\text { SA-HRP }\end{array}$ & $\begin{array}{l}4 \mu \mathrm{g} / \mathrm{mL} \\
4 \mathrm{ng} / \mathrm{mL} \\
1 \mu \mathrm{g} / \mathrm{mL} \\
1: 2000 \text { dilution }\end{array}$ & $\begin{array}{l}500 \mu \mathrm{g} / \mathrm{mL} \\
400 \mathrm{ng} / \mathrm{mL} \\
500 \mu \mathrm{g} / \mathrm{mL}\end{array}$ & $\begin{array}{l}0.1 \mathrm{M} \mathrm{Na}_{2} \mathrm{HPO}_{4}, \mathrm{pH} 6.0 \\
5 \% \mathrm{FCS} / \mathrm{PBS} \\
5 \% \mathrm{FCS} / \mathrm{PBS} \\
5 \% \mathrm{FCS} / \mathrm{PBS}\end{array}$ & $\begin{array}{l}\text { BD Pharmingen } \\
\text { BD Pharmingen } \\
\text { BD Pharmingen }\end{array}$ \\
\hline $\lg M$ & $\begin{array}{l}\text { Capture } \\
\text { Top Standard } \\
\text { Detection } \\
\text { SA-HRP }\end{array}$ & $\begin{array}{l}1 \mu \mathrm{g} / \mathrm{mL} \\
250 \mathrm{ng} / \mathrm{mL} \\
500 \mathrm{ng} / \mathrm{mL} \\
1: 1000 \text { dilution }\end{array}$ & $\begin{array}{l}500 \mu \mathrm{g} / \mathrm{mL} \\
500 \mu \mathrm{g} / \mathrm{mL} \\
500 \mu \mathrm{g} / \mathrm{mL}\end{array}$ & $\begin{array}{l}0.1 \mathrm{M} \mathrm{Na}_{2} \mathrm{HPO}_{4}, \mathrm{pH} 9.0 \\
5 \% \mathrm{FCS} / \mathrm{PBS} \\
5 \% \mathrm{FCS} / \mathrm{PBS} \\
5 \% \mathrm{FCS} / \mathrm{PBS}\end{array}$ & $\begin{array}{l}\text { BD Pharmingen } \\
\text { BD Pharmingen } \\
\text { BD Pharmingen } \\
\text { BD Pharmingen }\end{array}$ \\
\hline
\end{tabular}

* via Thermo Fisher Scientific 


\subsubsection{Flow cytometry antibodies and dyes}

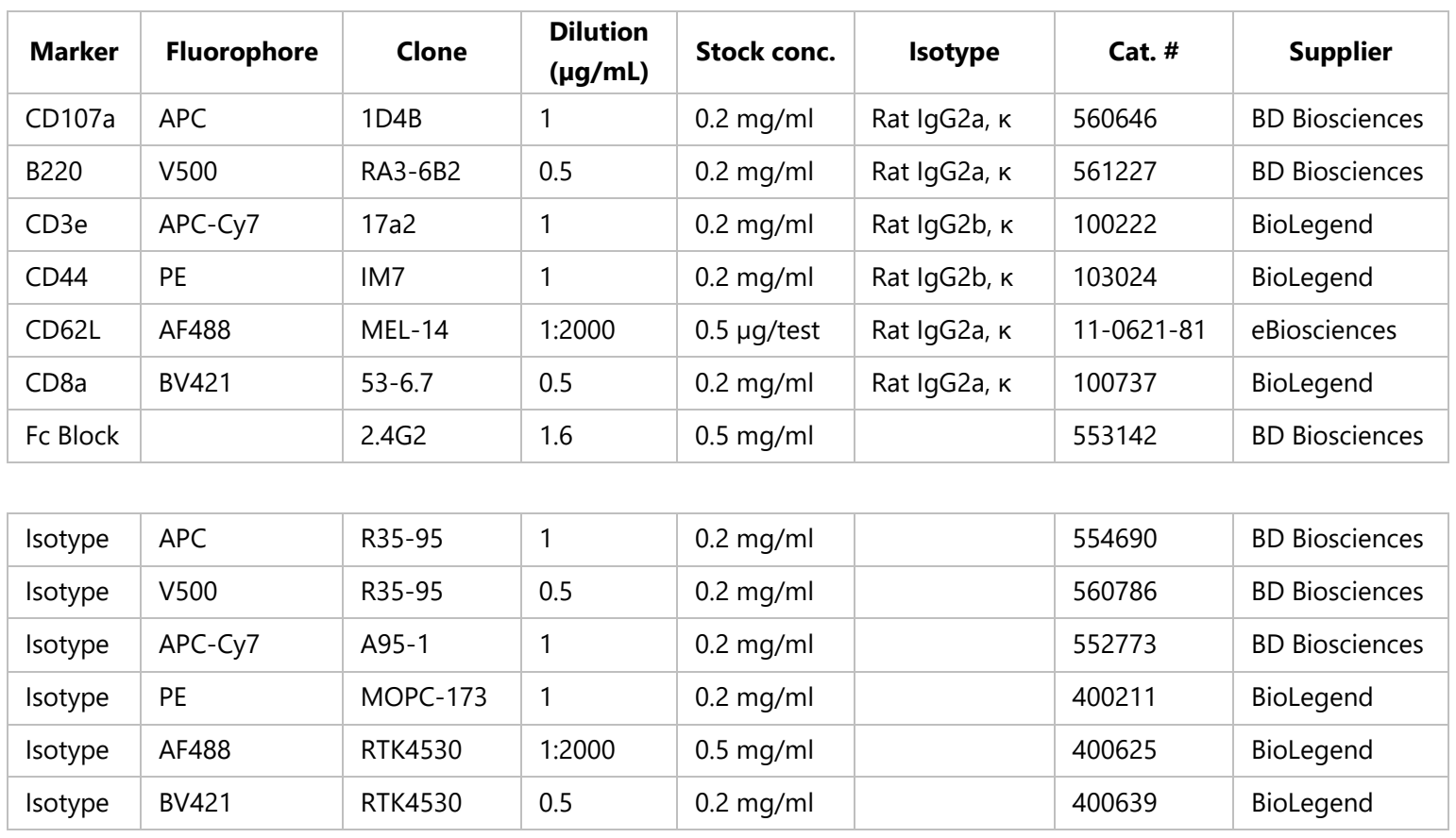

\section{Other flow reagents}

Zombie NIR viability dye

pHrodo $^{\text {TM Red E. coli BioParticles }}{ }^{\text {TM }}$ Conjugate for Phagocytosis CFSE

$\begin{array}{ll}\text { Cat \# } & \text { Supplier } \\ 423105 & \text { BioLegend } \\ \text { P35361 } & \text { Molecular Probes (via ThermoFisher) } \\ \text { C34570 } & \text { Thermo Fisher Scientific }\end{array}$




\subsection{Supplementary material for Chapter 3}

\subsubsection{Gating strategy: Phagocytosis by RAW 264.7 cells}
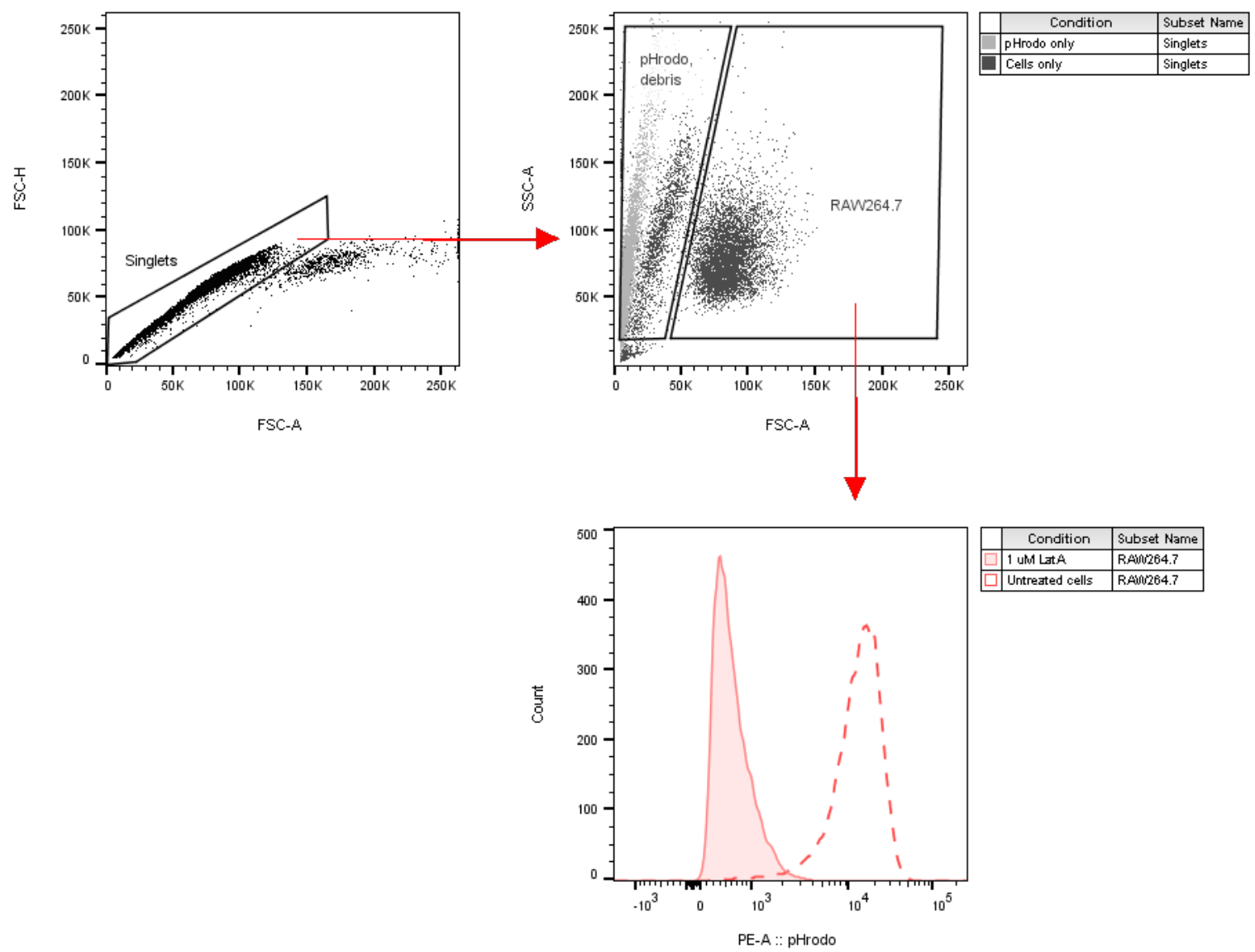

Figure 9.1. Gating strategy to measure phagocytosis by RAW 264.7 by flow cytometry

After doublet exclusion, the larger size of RAW264.7 cells were used to identify these cells of interest and a gate was drawn to exclude debris and pHrodo bioparticles. From the RAW264.7 gate, pHrodo fluorescence intensity was measured by the PE laser/filter set (586/42 Filter). Higher fluorescence intensity indicates phagocytosis ability, seen with untreated cells (dotted); pHrodo (PE) signal decreases with treatment of $1 \mu \mathrm{M}$ LatA, a known inhibitor of phagocytosis. 


\subsubsection{Gating strategy: B and T cell proliferation with CFSE}
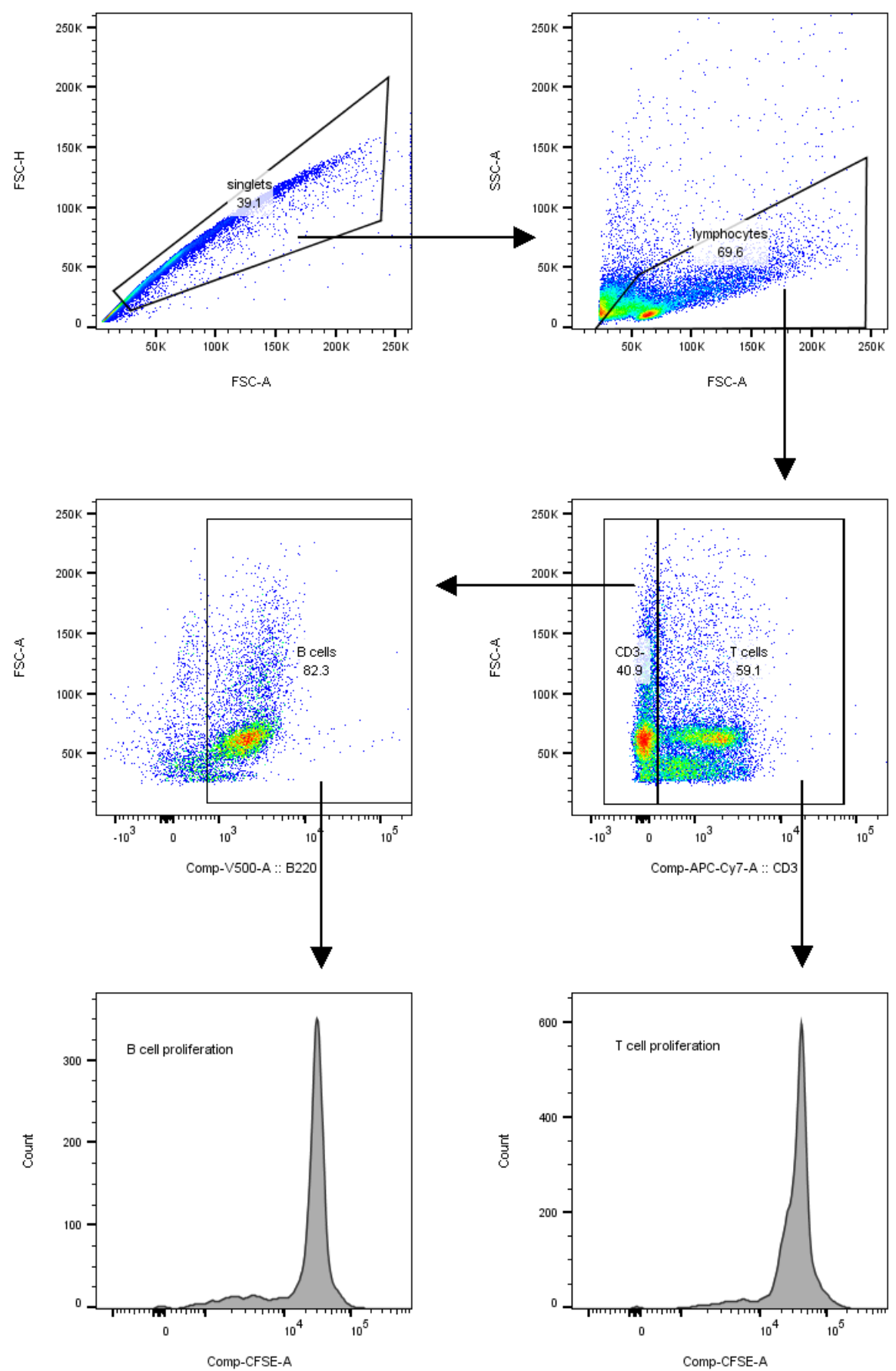

Figure 9.2. Gating strategy to assess $B$ and $T$ cell proliferation during activation

After doublet exclusion, lymphocytes were identified by FSC and SSC. CD3 ${ }^{+}$cells were identified as T cells, and CFSE intensity was measured using the FITC channel (527/32 filter). B220 ${ }^{+}$cells from the CD3 ${ }^{-}$gate were identified as B cells, and CFSE intensity from these cells were measured as above. 


\subsubsection{Gating strategy: to identify subpopulations during eCTL generation}
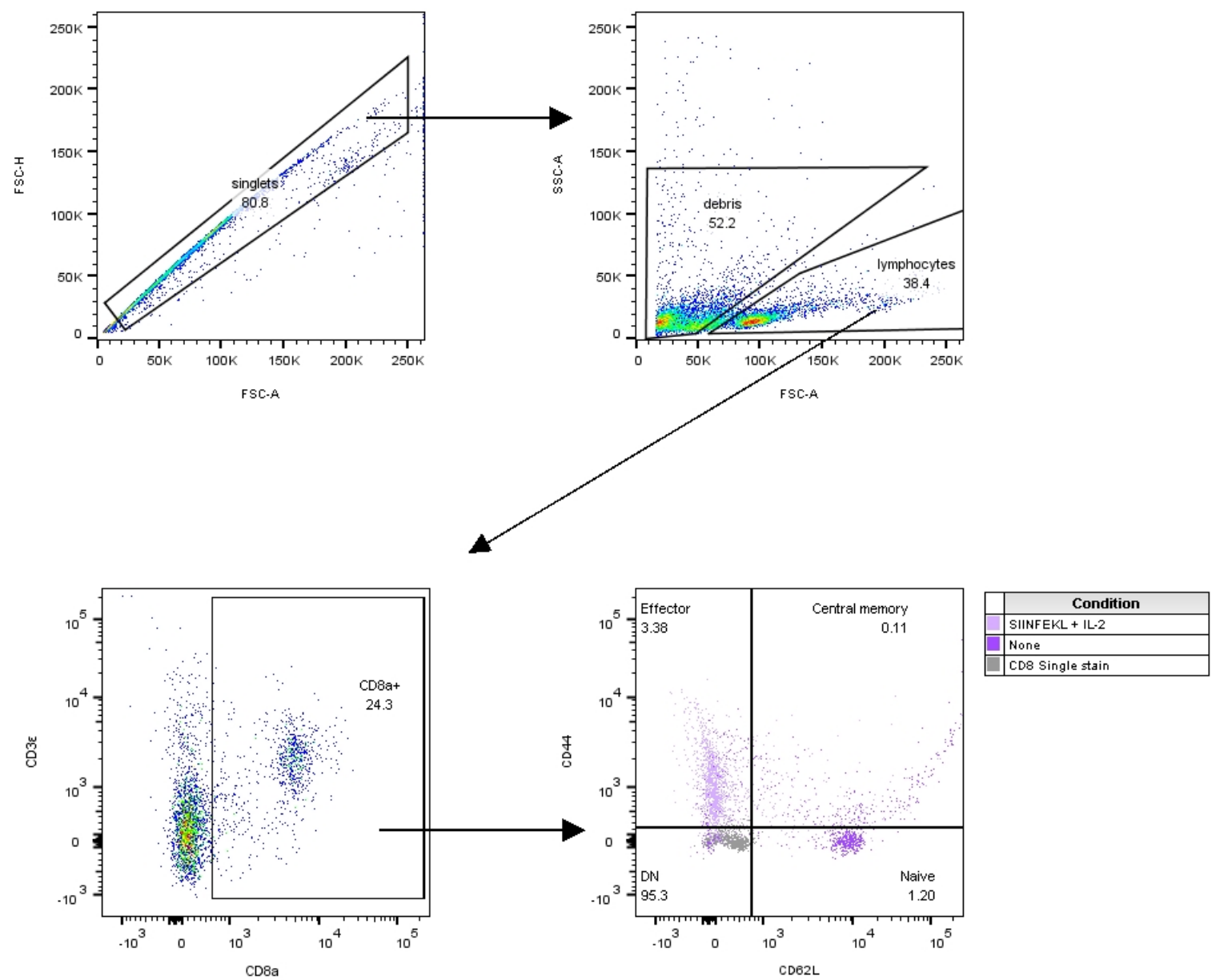

Figure 9.3. Gating strategy to assess priming efficacy when generating effector CTL

After doublet exclusion, lymphocytes were identified by FSC and SSC. CD3 ${ }^{+} \mathrm{CD}^{+} \mathrm{CTL}$ sere identified from the lymphocyte gate. Subpopulations of activated CTLs were identified by CD62L and CD44 surface

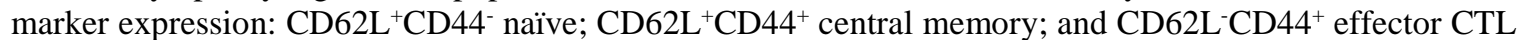
were assessed as done by Oehen and Brduscha-Riem (1998). 


\subsubsection{Gating strategy: Identifying eCTL by FCS and SSC}

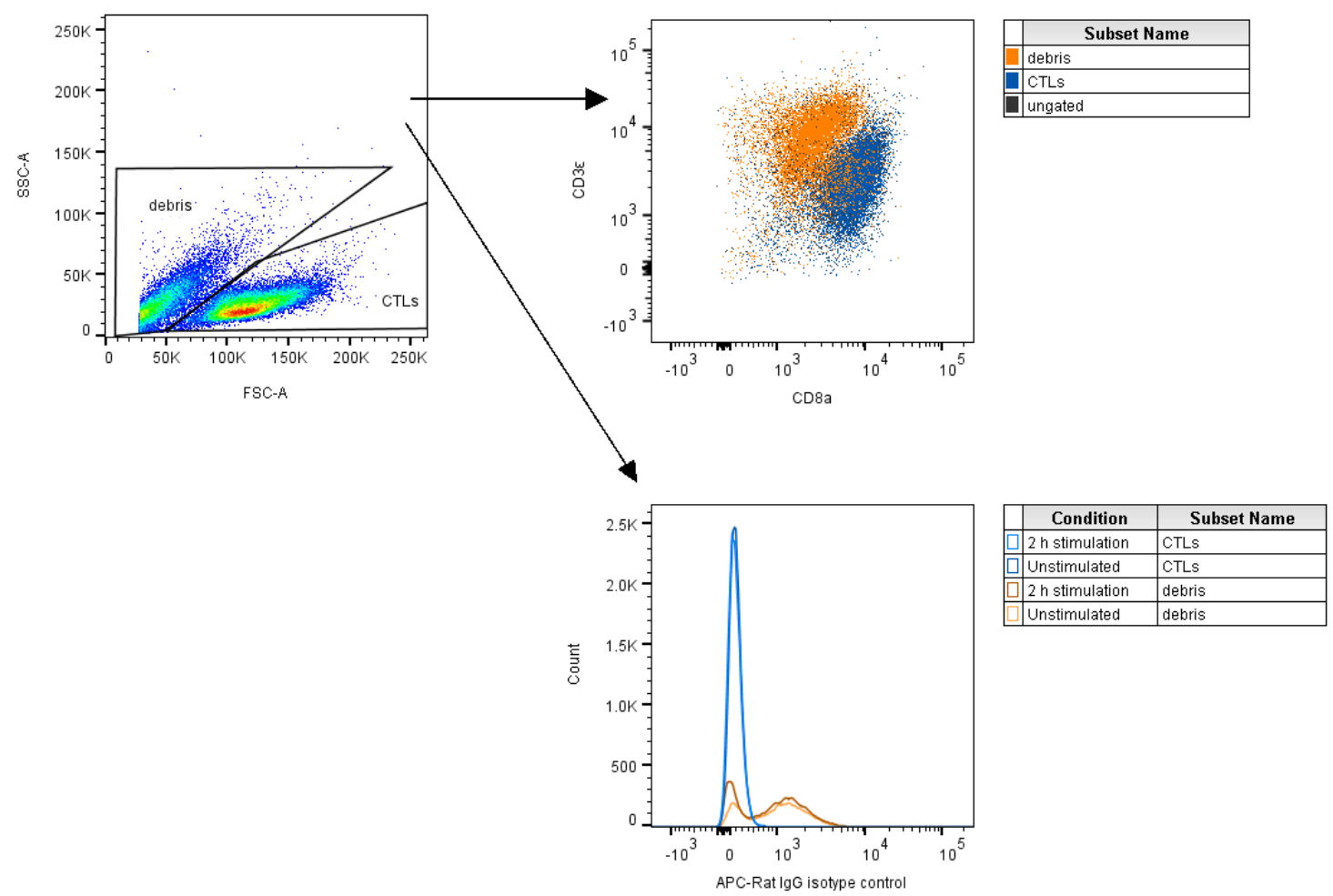

Figure 9.4. eCTLs can be determined by their forward- and side-scatter properties

Samples of effector CTLs were stained for CD3 and CD8 to identify CTLs from the splenocyte culture. After singlet exclusion, lymphocytes were identified by forward-scatter (FSC) and side-scatter (SSC) properties. CD3 and CD8 measurements indicate the lymphocyte gate accurately captured CTLs. Positive staining for isotype controls confirm the debris gate.

Including markers (upper right) did not add any information of interest. $\mathrm{CD}^{+} \mathrm{CD}^{+} \mathrm{CTLs}$ were sufficiently identified by FSC vs SSC morphology (left). Looking at the lymphocyte events (blue) in the upper right plot shows most events in this gate were $\mathrm{CD}^{+} \mathrm{CD}^{+}$. As mentioned in the main text, the $\mathrm{CD}^{+}$component of the lymphocyte gate averaged to $98.3 \%$ across 50 samples, so staining for this marker was deemed unnecessary: it only added reagent costs and time to the assay. 


\subsection{Supplementary material for Chapter 4}

\subsubsection{Immunomodulation by screen-building set compounds in BMDM}

In this experiment, BMDM were activated in the presence of the seven compounds used in chapter 4, the screen building compound set. This was done to assess any differences between compound-induced alterations to RAW 264.7 cells and BMDM cells upon activation. As explained in chapter 4, these cells respond differently to treatments compared to RAW 264.7 cells. 

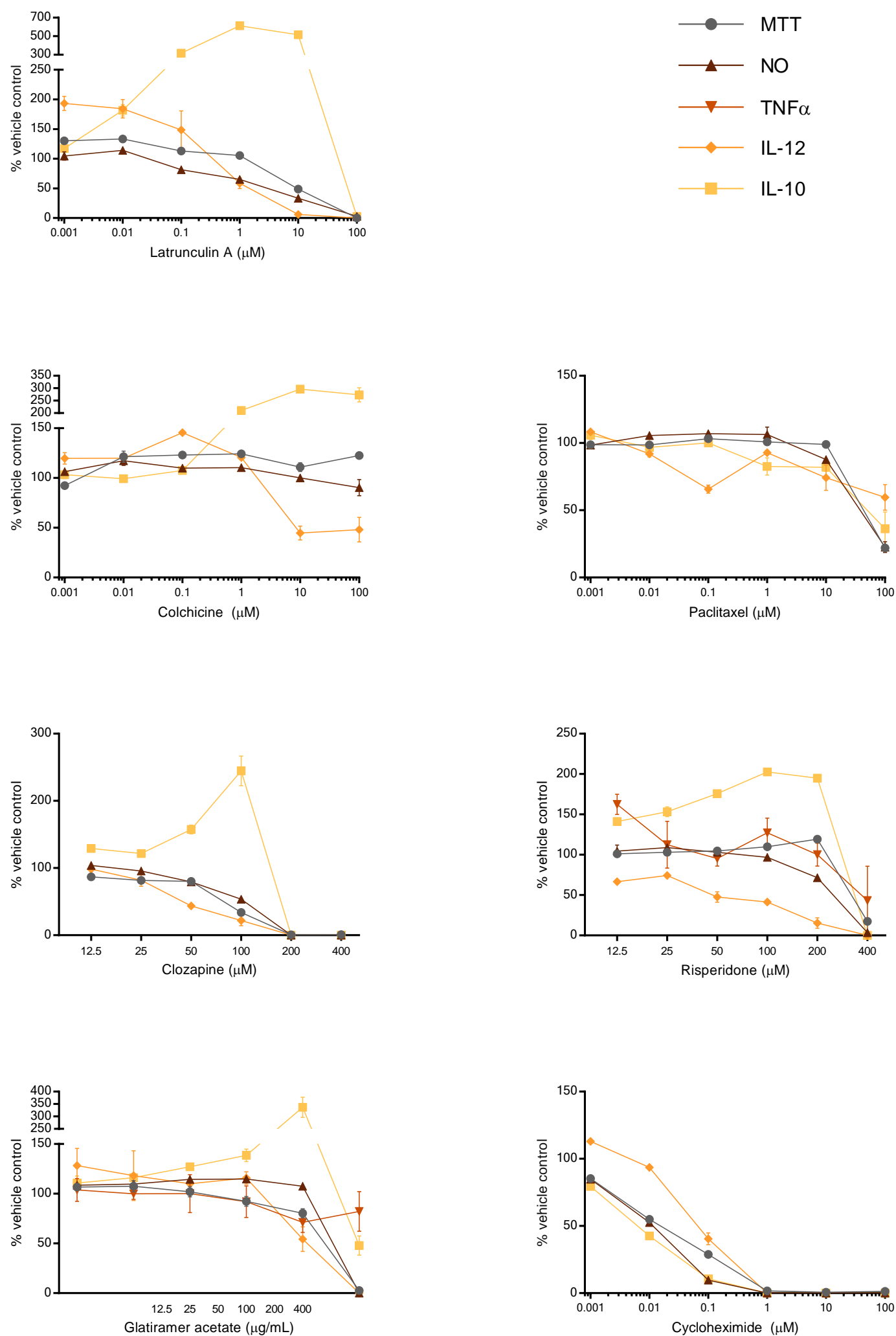

Figure 9.5. BMDM activation in the presence of screen-building set compounds

$100,000 \mathrm{BMDM}$ were seeded into wells and stimulated with $20 \mathrm{U} / \mathrm{ML}$ IFN $\gamma$ for $18 \mathrm{~h}$. Indicated treatments were added alongside $200 \mathrm{ng} / \mathrm{mL}$ LPS to activate macrophages. After $24 \mathrm{~h}$, cell viability was assessed by MTT and cytokines were measured from supernatants by ELISA. NO production was assessed by Griess reaction. Data are means and SEM from one experiment, conducted in duplicate. Risperidone data presented here are the same as presented in Figure 3.4. 


\subsubsection{Assessing macrophage viability with MTT and Zombie dyes}
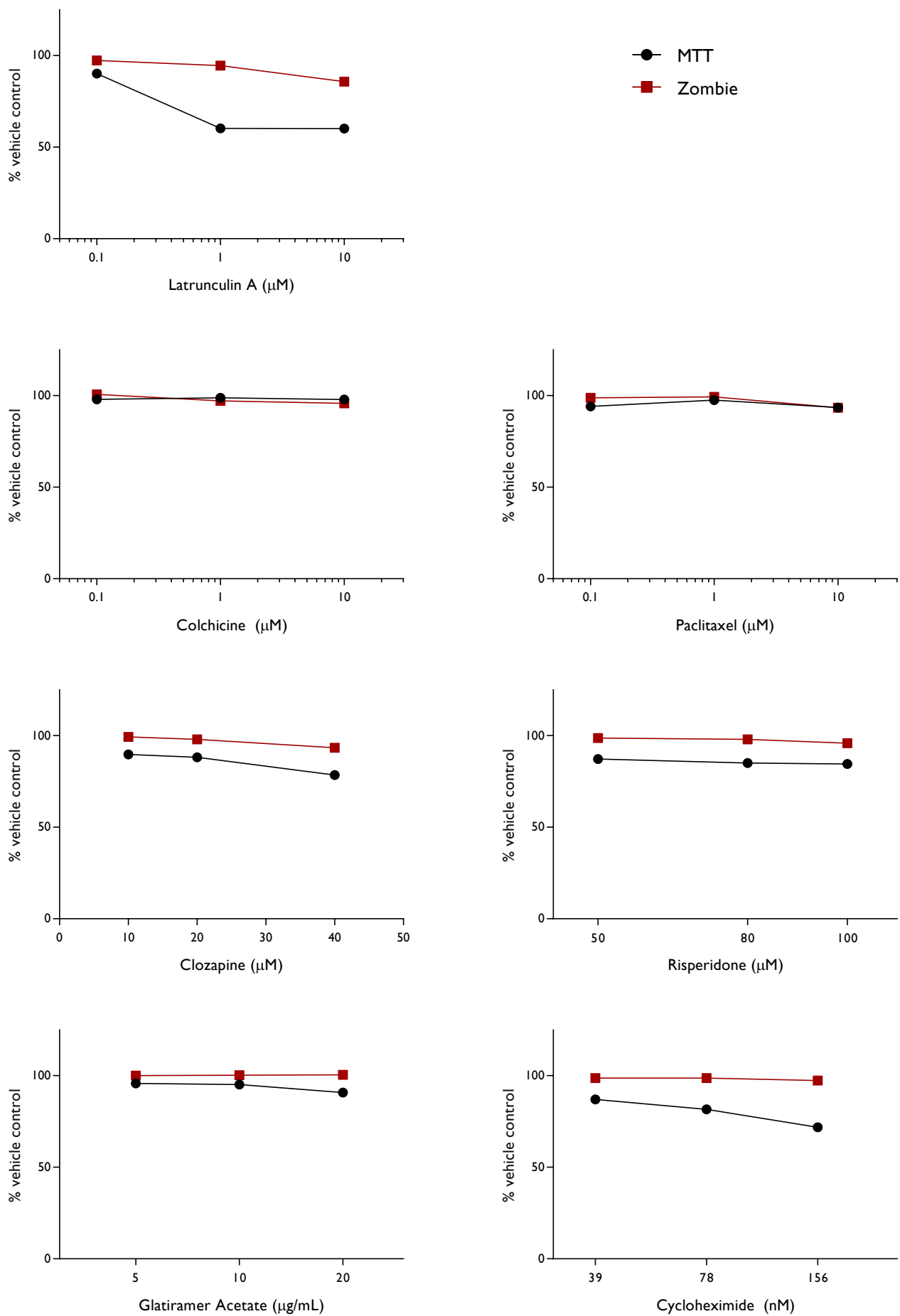

Figure 9.6. Assessment of cell viability by Zombie dyes and MTT

100,000 RAW 264.7 cells were seeded into wells and treated for eight hours with the indicated treatments. Cells were then assessed for viability via MTT assay or by the addition of 1:1000 Zombie dye. Zombiestained cells were assessed via flow cytometry, and the proportion of dead cells, identified by positive staining, for each treatment condition was compared to vehicle equivalents. Data are means from one experiment conducted in duplicate. 


\subsubsection{The effects of compounds on B cell viability}
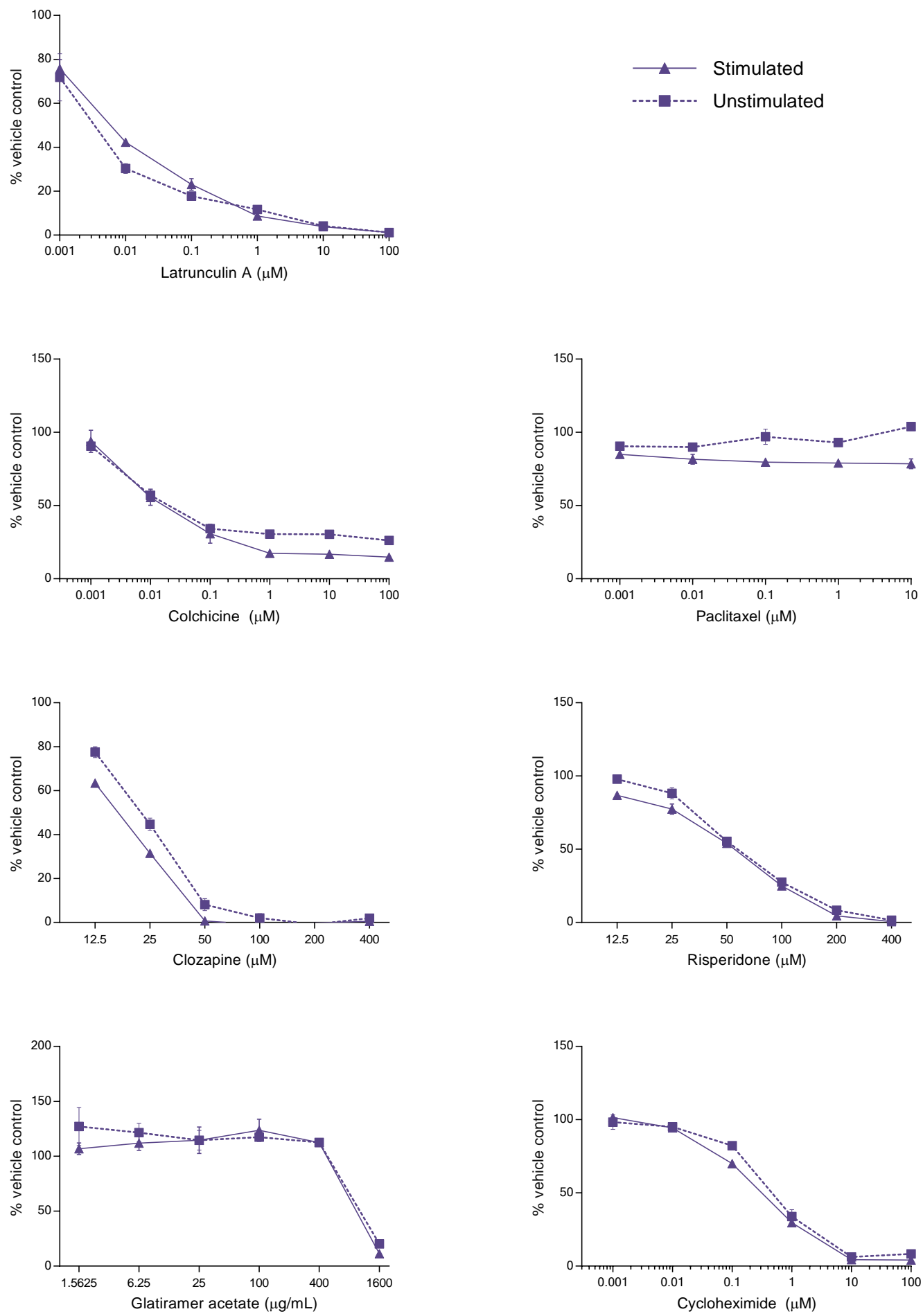

Figure 9.7. Compounds were similarly toxic to stimulated and unstimulated B cells

$1 \times 10^{6}$ splenocytes were seeded into wells of a 96-well plate and were treated with indicated compounds. Cells were stimulated with $0.1 \mu \mathrm{g} / \mathrm{mL}$ LPS, or remained unstimulated for $48 \mathrm{~h}$ before viability was assessed by MTT. Data are means and SEM from one experiment conducted in duplicate. 
9.5 Supplementary material for Chapter 5 


\subsubsection{Compound A}

\subsubsection{Concentration selection for compound A}

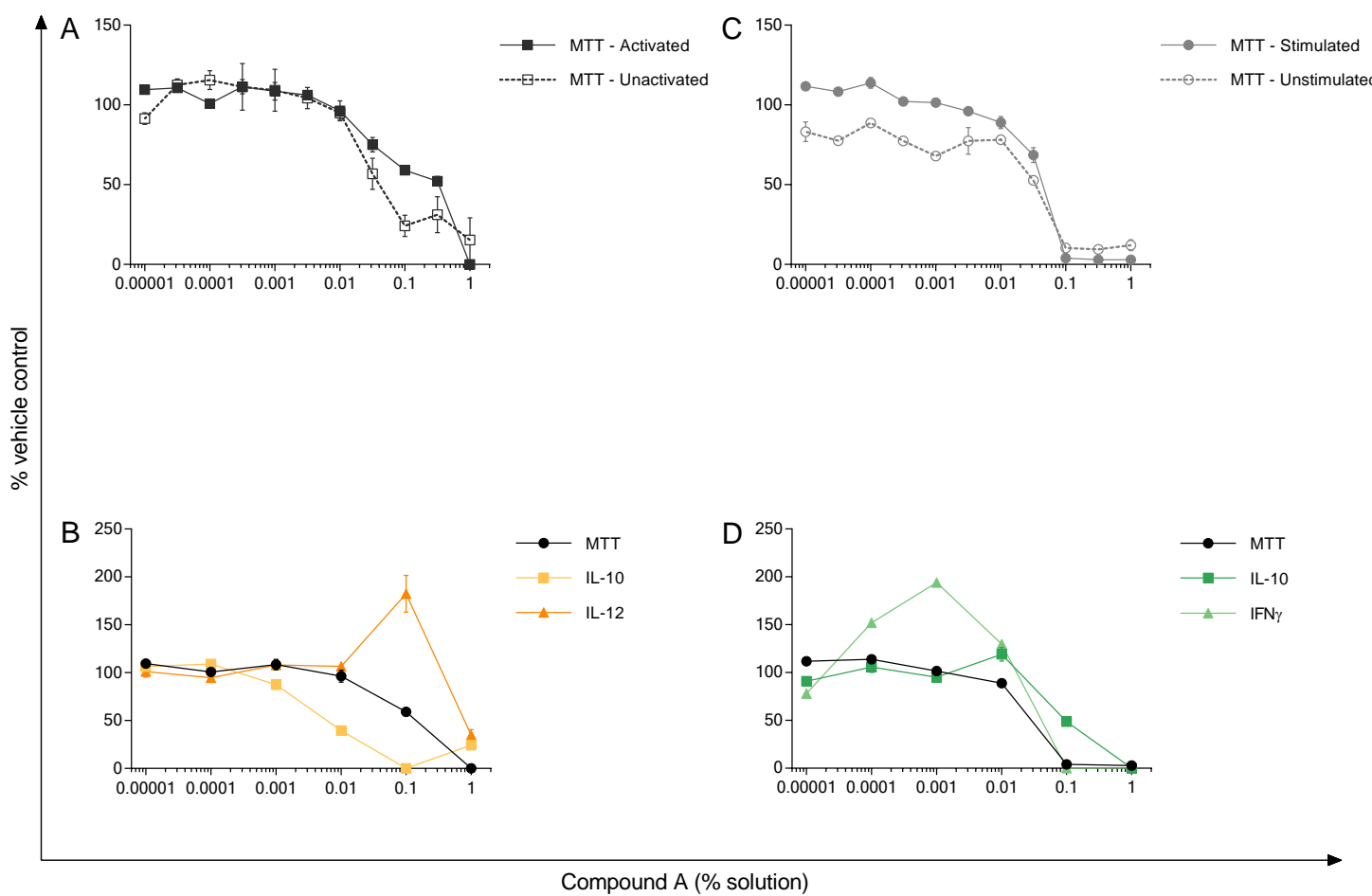

Figure 9.8. Concentration identification for compound A using stimulated macrophages and Th cells Activated macrophages were treated with Compound A and viability (A) and IL-10 and IL-12 (B) was assessed. Similarly, Helper T cells were activated in the presence of Compound A to generate MTT (C) and IL-10 and IFN $\gamma$ data (D). Data were used to select concentrations for further testing as described in (Ch 3, section 5.3.1)

Concentrations of compound A to use through the screening strategy were identified from macrophage activation and helper T cell activation assays. For macrophages, 0.001 and 0.01\% solutions were selected for their lack of toxicity seen during MTT analysis. These concentrations also alter cytokine production by macrophages. The $\mathrm{IC}_{20}$ concentration was mistakenly omitted. For splenocytes, 0.001 and $0.01 \%$ solutions were also selected for non-toxicity. These concentrations also alter IFN $\gamma$ production by Th cells. The additional $\mathrm{IC}_{20}$ concentration of $0.035 \%$ was also selected to test. 


\subsubsection{Screening data for Compound A}

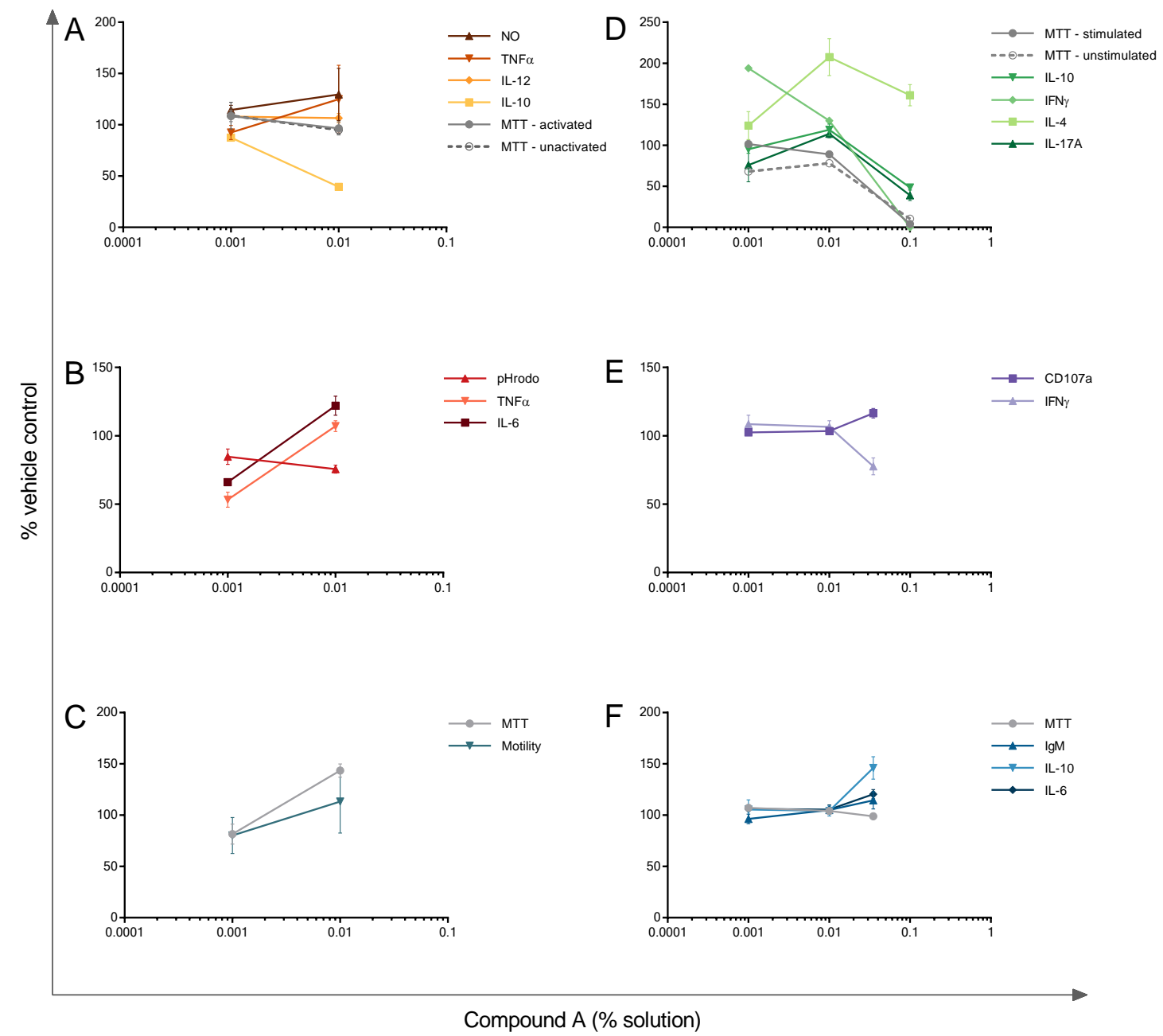

Figure 9.9. Screen outputs for Compound A

Compound A, at indicated concentrations, was run through the six assays. Macrophage activation (A), phagocytosis (B) and motility (C) are macrophage-based assays. In the right column are the splenocyte-based assays: helper T cell activation (D), CTL degranulation (E) and B cell activity (F). Experiments were conducted as explained in thesis methods (Ch 2). Shown are compound-induced changes compared to vehicle controls (i.e. \% vehicle). Closure in vehicle-treated wells during the motility assay averaged 74.0\% (SD = 15.9\%). Data are means and SEM from each experiment which was conducted once in duplicate. 


\subsubsection{Compound B}

\subsubsection{Concentration selection for compound B}

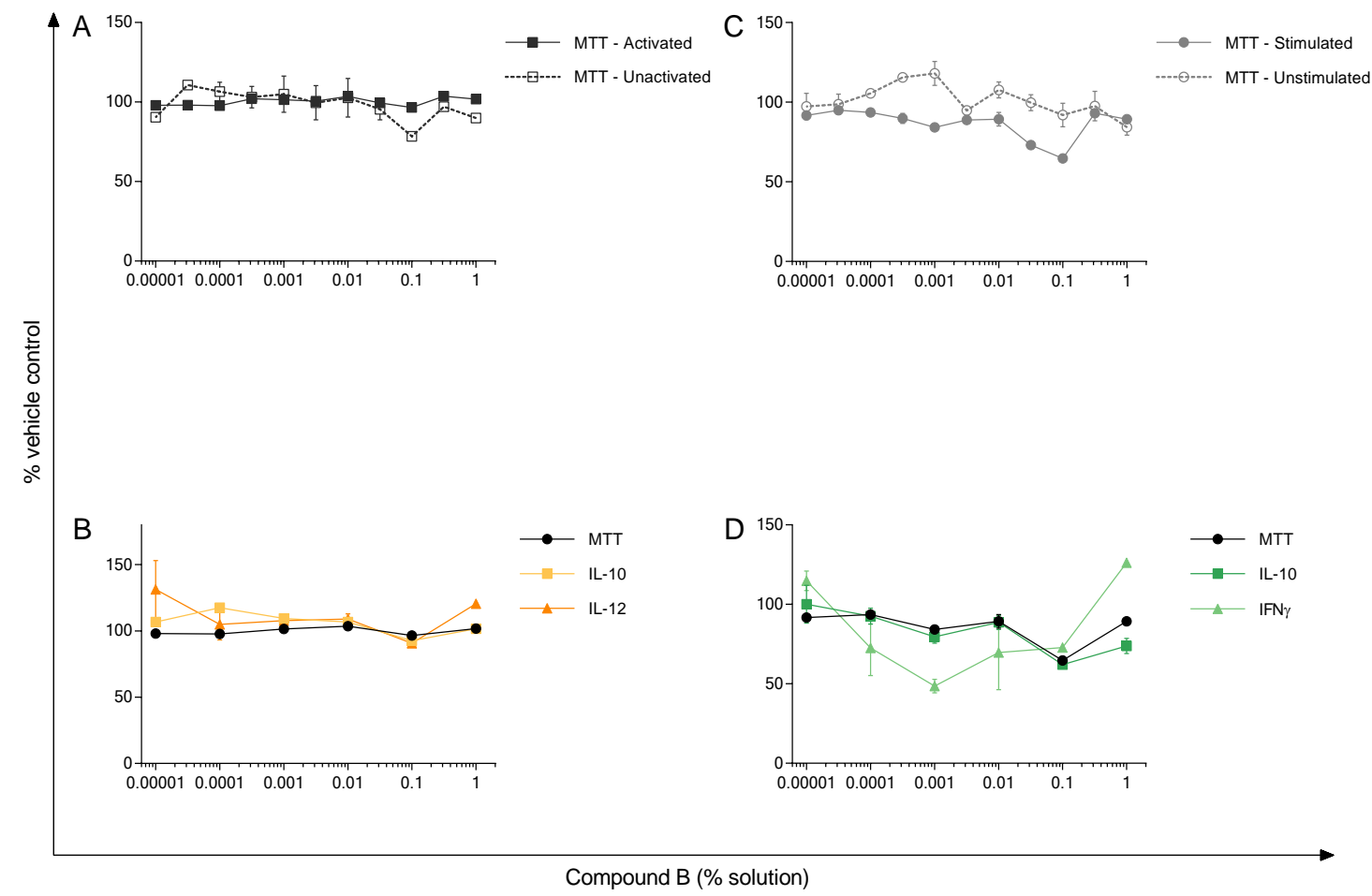

Figure 9.10. Concentration identification for compound B using stimulated macrophages and Th cells Activated macrophages were treated with Compound B and viability (A) and IL-10 and IL-12 (B) was assessed. Similarly, Helper T cells were activated in the presence of Compound B to generate MTT (C) and IL-10 and IFN $\gamma$ data (D). Data were used to select concentrations for further testing as described in (Ch 3, section 5.3.1)

Concentrations of compound B to use through the screening strategy were identified from macrophage activation and helper $\mathrm{T}$ cell activation assays.

Compound B did not demonstrate toxicity on either cell type with the concentrations tested. Because no interested changes to macrophage cytokine production was observed, three high concentrations (0.01, 0.1 and 1\% solutions) were selected for testing. For splenocytes, the concentration-dependent changes to IFN $\gamma$ production encouraged the selection of four high concentrations for testing (0.001, $0.01,0.1$ and $1 \%$ solutions). 


\subsubsection{Screening data for Compound B}

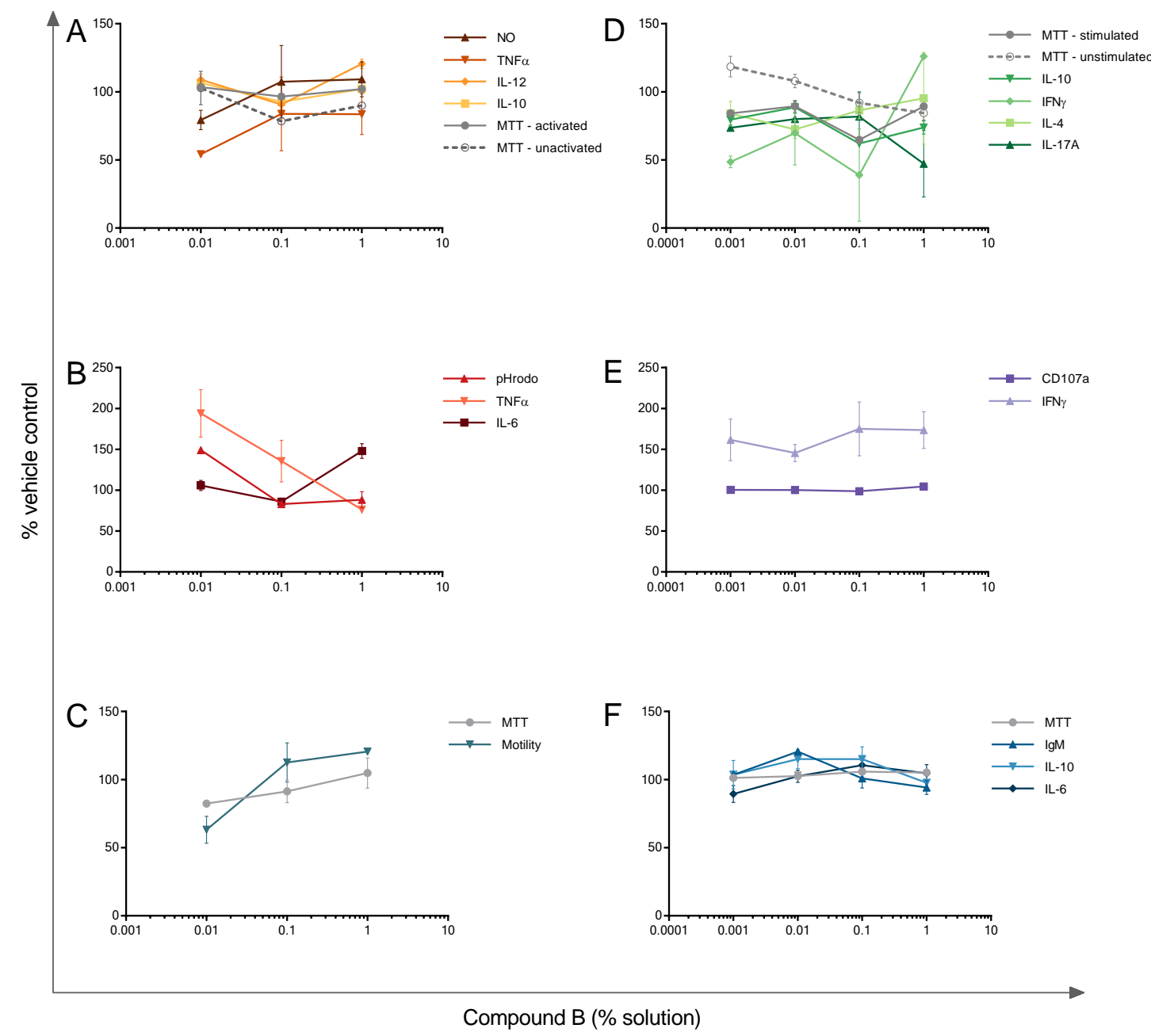

Figure 9.11. Screen outputs for Compound B

Compound $\mathrm{B}$, at indicated concentrations, was run through the six assays. Macrophage activation (A), phagocytosis (B) and motility (C) are macrophage-based assays. In the right column are the splenocyte-based assays: helper T cell activation (D), CTL degranulation (E) and B cell activity (F). Experiments were conducted as explained in thesis methods (Ch 2). Shown are compound-induced changes compared to vehicle controls (i.e. \% vehicle). Closure in vehicle-treated wells during the motility assay averaged $74.0 \%$ (SD = 15.9\%). Data are means and SEM from each experiment which was conducted once in duplicate. 


\subsubsection{Compound C}

\subsubsection{Concentration selection for compound C}

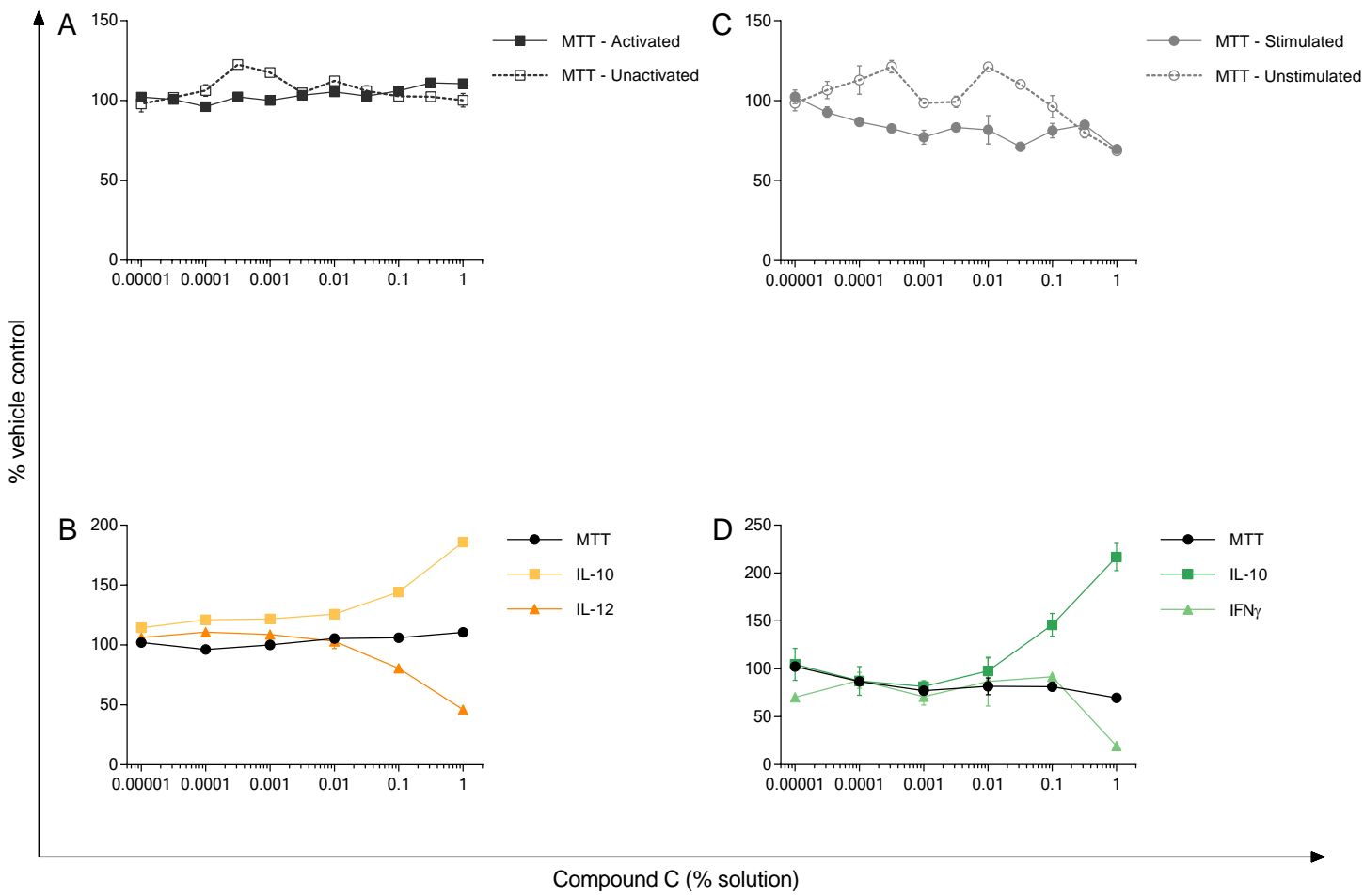

Figure 9.12. Concentration identification for compound $\mathrm{C}$ using stimulated macrophages and Th cells Activated macrophages were treated with Compound C and viability (A) and IL-10 and IL-12 (B) was assessed. Similarly, Helper T cells were activated in the presence of Compound C to generate MTT (C) and IL-10 and IFN $\gamma$ data (D). Data were used to select concentrations for further testing as described in (Ch 3, section 5.3.1)

Concentrations of compound $\mathrm{C}$ to use through the screening strategy were identified from macrophage activation and helper $\mathrm{T}$ cell activation assays.

No significant cytotoxic effects were seen in macrophages or splenocytes treated with compound C. Here, concentrations were selected based on changes to cytokine production. For both these cell types, $0.01,0.1$ and $1 \%$ solutions were investigated as these concentrations alter macrophage activation, and promote IL-10 and reduce IFN $\gamma$ production in activated Th cells. 


\subsubsection{Screening data for Compound C}

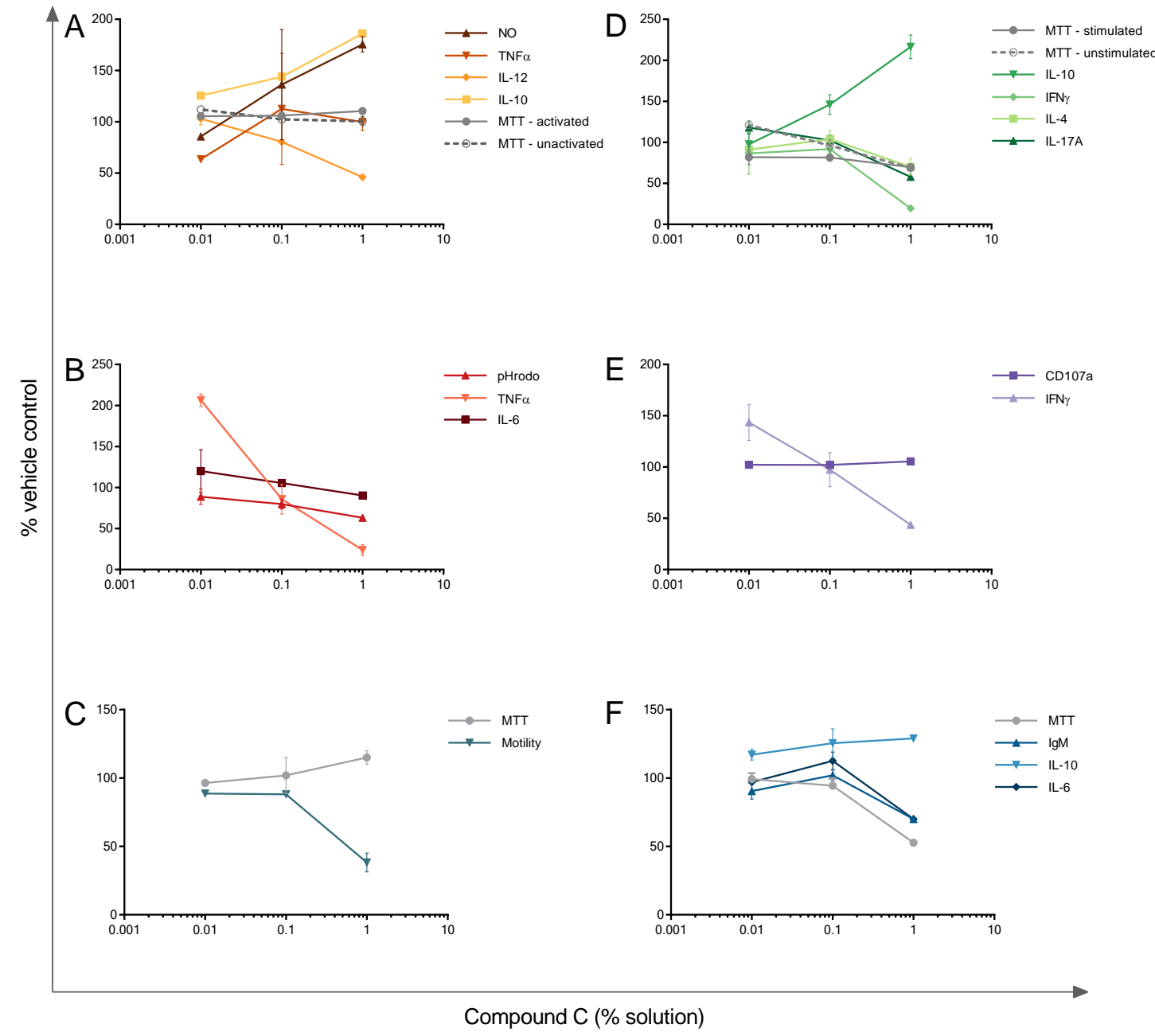

Figure 9.13. Screen outputs for Compound C

Compound $\mathrm{C}$, at indicated concentrations, was run through the six assays. Macrophage activation (A), phagocytosis (B) and motility (C) are macrophage-based assays. In the right column are the splenocyte-based assays: helper T cell activation (D), CTL degranulation (E) and B cell activity (F). Experiments were conducted as explained in thesis methods (Ch 2). Shown are compound-induced changes compared to vehicle controls (i.e. \% vehicle). Closure in vehicle-treated wells during the motility assay averaged $74.0 \%$ (SD = 15.9\%). Data are means and SEM from each experiment which was conducted once in duplicate. 


\subsubsection{Compound D}

\subsubsection{Concentration selection for compound D}

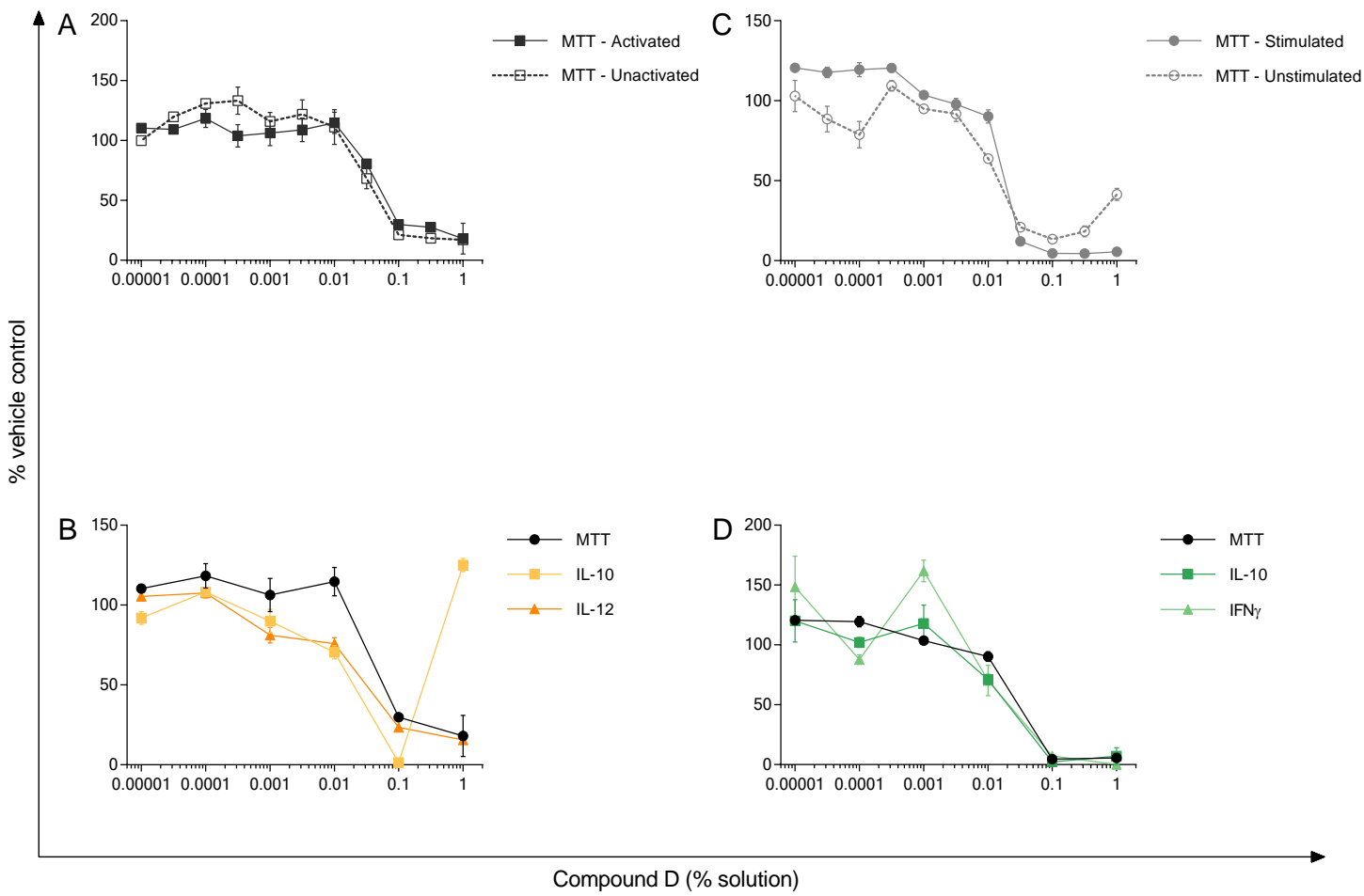

Figure 9.14. Concentration identification for compound $D$ using stimulated macrophages and Th cells Activated macrophages were treated with Compound D and viability (A) and IL-10 and IL-12 (B) was assessed. Similarly, Helper T cells were activated in the presence of Compound D to generate MTT (C) and IL-10 and IFN $\gamma$ data (D). Data were used to select concentrations for further testing as described in (Ch 3, section 5.3.1)

Concentrations of compound D to use through the screening strategy were identified from macrophage activation and helper $\mathrm{T}$ cell activation assays.

Concentrations of compound D were identified by cytotoxicity data for both macrophages and splenocytes. An $\mathrm{IC}_{20}$ value could not be calculated for macrophages, due to the lack of plateau at high concentrations, so 0.001 and $0.01 \%$ solutions were selected as these concentrations were non-toxic. For splenocytes, the $\mathrm{IC}_{20}$ was elicited with a $0.009 \%$ solution. $0.01 \%$ was selected as it is close to this $\mathrm{IC}_{20}$ value but falls on a 10 -fold dilution. 0.001 and $0.0001 \%$ solutions were added for their interesting effect on splenocyte IFN $\gamma$ production and relative toxicity on unstimulated T cells respectively. 


\subsubsection{Screening data for Compound D}

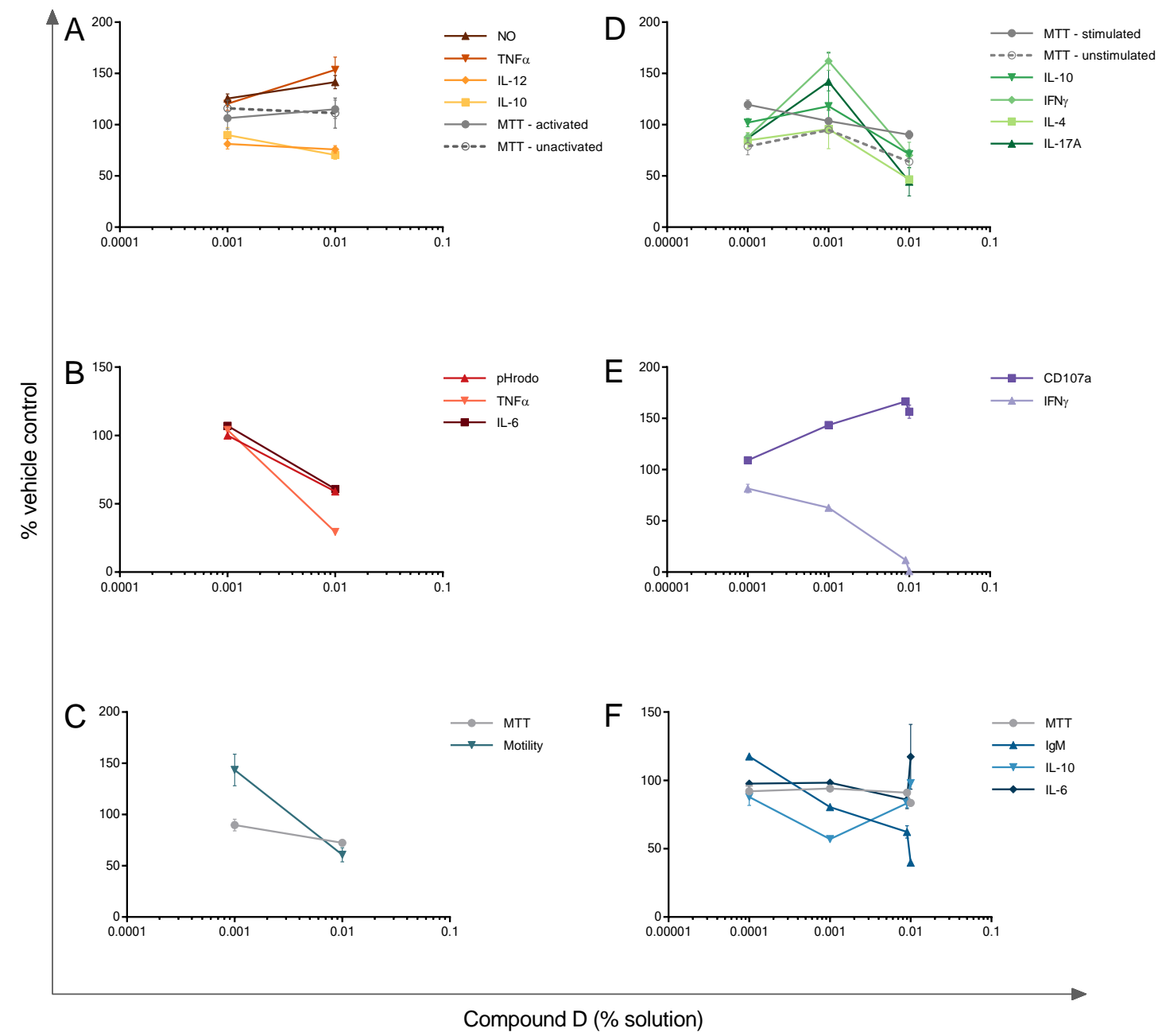

Figure 9.15. Screen outputs for Compound D

Compound D, at indicated concentrations, was run through the six assays. Macrophage activation (A), phagocytosis (B) and motility (C) are macrophage-based assays. In the right column are the splenocyte-based assays: helper T cell activation (D), CTL degranulation (E) and B cell activity (F). Experiments were conducted as explained in thesis methods (Ch 2). Shown are compound-induced changes compared to vehicle controls (i.e. \% vehicle). Closure in vehicle-treated wells during the motility assay averaged $74.0 \%$ (SD = 15.9\%). Data are means and SEM from each experiment which was conducted once in duplicate. 


\subsubsection{Compound E}

\subsubsection{Concentration selection for compound $\mathrm{E}$}

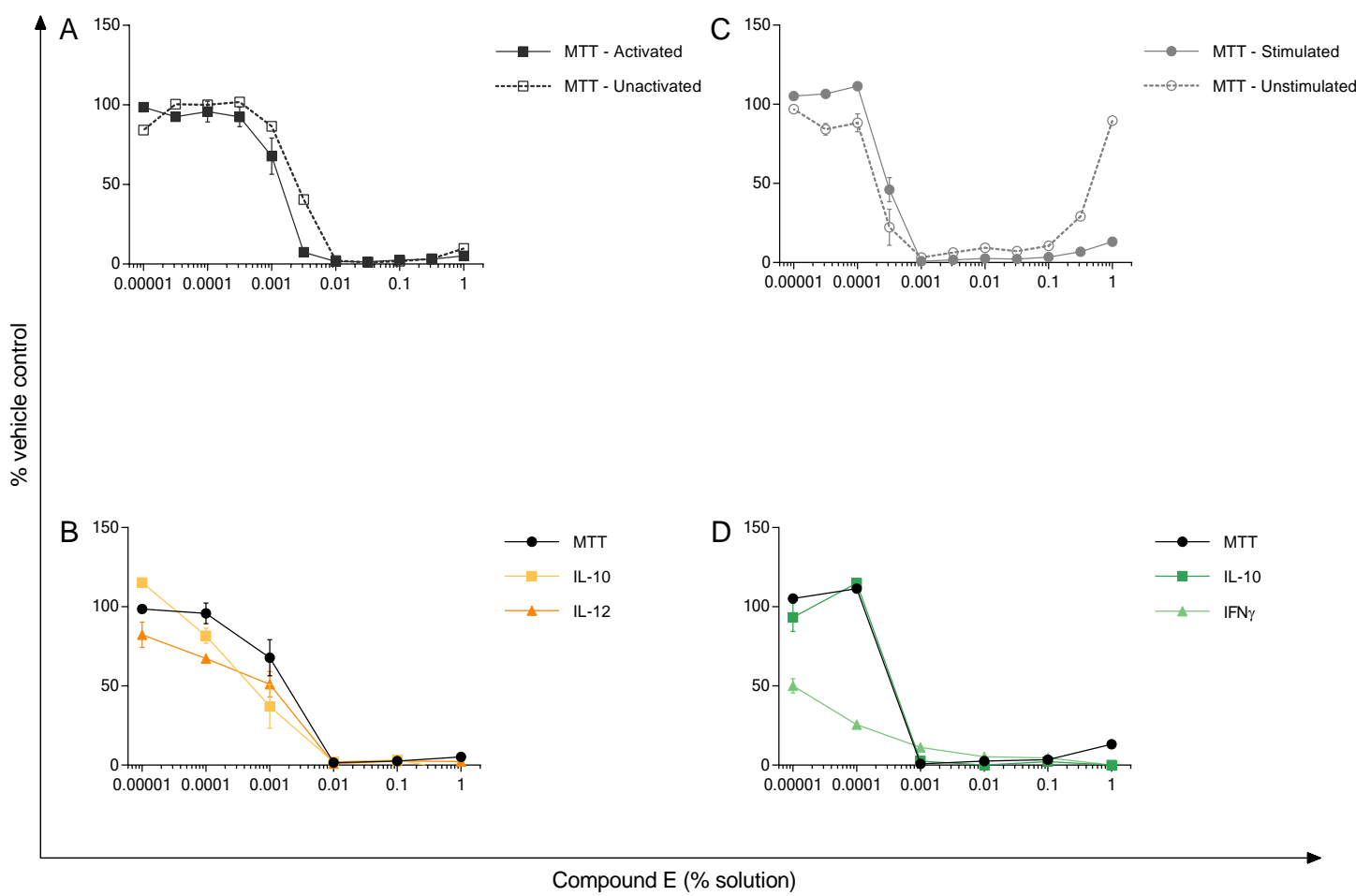

Figure 9.16. Concentration identification for compound $\mathrm{E}$ using stimulated macrophages and Th cells Activated macrophages were treated with Compound E and viability (A) and IL-10 and IL-12 (B) was assessed. Similarly, Helper T cells were activated in the presence of Compound E to generate MTT (C) and IL-10 and IFN $\gamma$ data (D). Data were used to select concentrations for further testing as described in (Ch 3, section 5.3.1)

Concentrations of compound $\mathrm{E}$ to use through the screening strategy were identified from macrophage activation and helper $\mathrm{T}$ cell activation assays.

Compound $\mathrm{E}$ has cytotoxic effects on both cell types, for this reason $\mathrm{IC}_{20}$ values could be calculated. For macrophages, the $\mathrm{IC}_{20}$ is seen with a $0.0016 \%$ solution. 0.001 and $0.0001 \%$ solutions were included as they altered macrophage cytokine production. For splenocytes the $\mathrm{IC}_{20}$ was $0.00016 \%$. 0.0001 and $0.00001 \%$ were included due to the decrease in IFN $\gamma$ production by Th cells caused by these concentrations of compound. 


\subsubsection{Screening data for Compound E}

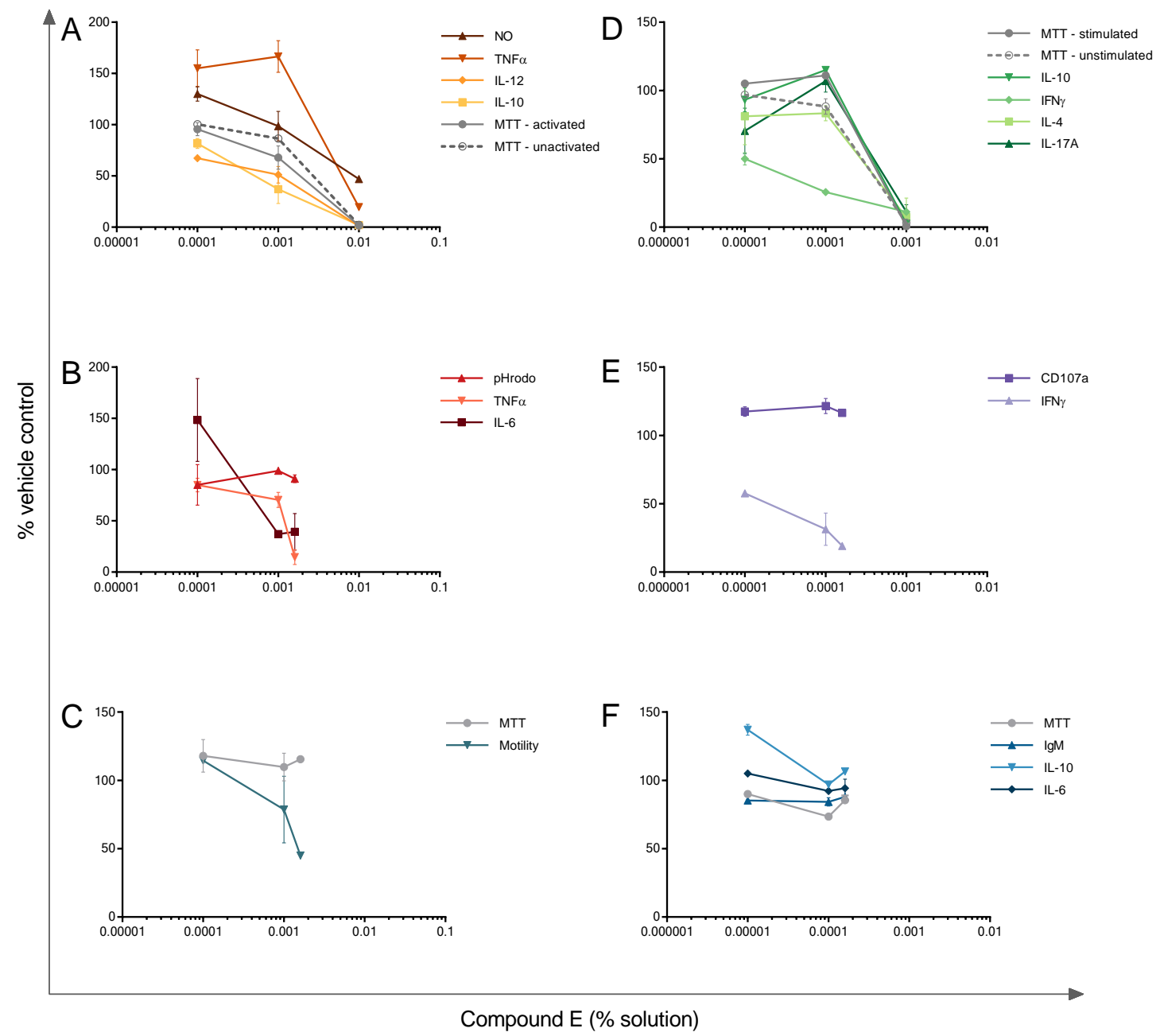

Figure 9.17. Screen outputs for Compound E

Compound E, at indicated concentrations, was run through the six assays. Macrophage activation (A), phagocytosis (B) and motility (C) are macrophage-based assays. In the right column are the splenocyte-based assays: helper T cell activation (D), CTL degranulation (E) and B cell activity (F). Experiments were conducted as explained in thesis methods (Ch 2). Shown are compound-induced changes compared to vehicle controls (i.e. \% vehicle). Closure in vehicle-treated wells during the motility assay averaged $74.0 \%$ (SD = 15.9\%). Data are means and SEM from each experiment which was conducted once in duplicate. 


\subsubsection{Compound F}

\subsubsection{Concentration selection for compound $F$}

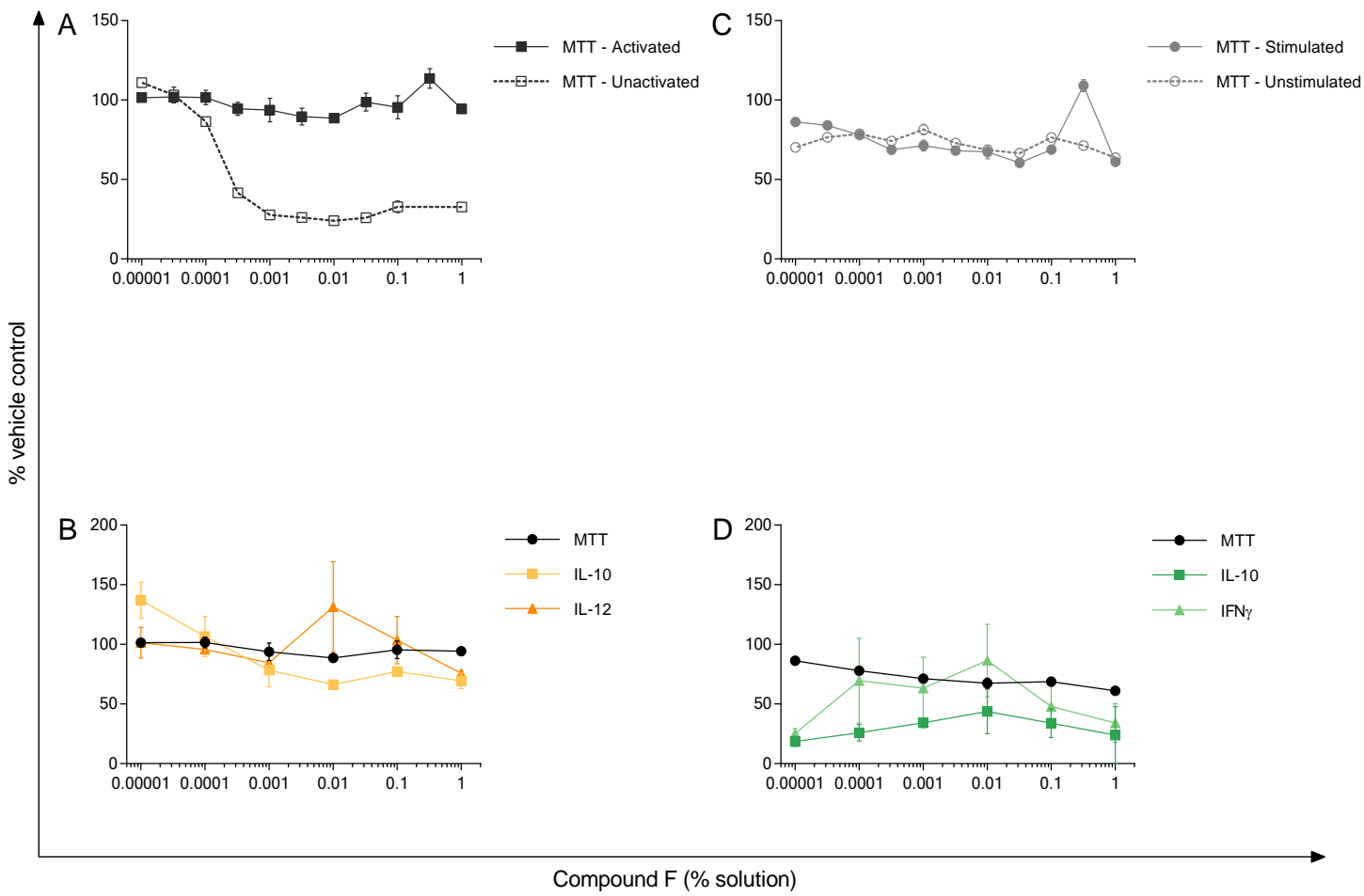

Figure 9.18. Concentration identification for compound $\mathrm{F}$ using stimulated macrophages and Th cells Activated macrophages were treated with Compound F and viability (A) and IL-10 and IL-12 (B) was assessed. Similarly, Helper T cells were activated in the presence of Compound F to generate MTT (C) and IL-10 and IFN $\gamma$ data (D). Data were used to select concentrations for further testing as described in (Ch 3, section 5.3.1)

Concentrations of compound $\mathrm{F}$ to use through the screening strategy were identified from macrophage activation and helper $\mathrm{T}$ cell activation assays.

Compound F had interesting cytotoxic effects on unstimulated macrophages, decreasing their viability. Activated macrophages were not affected. For this reason, 0.001\% and $0.0001 \%$ solutions were selected. The former concentrations cause starkly different effects between the two cell states, whilst the latter sees no difference. For splenocytes, three high concentrations (0.01, 0.1 and 1\%) of compound $\mathrm{F}$ were selected as no effects were seen to viability. Similarly, no concentration-dependant changes were seen to cytokine production: the decreased IFN $\gamma$ is seen with all concentrations. Because $0.316 \%$ solution caused a spike in stimulated Th cell MTT, it was included. 


\subsubsection{Screening data for Compound F}

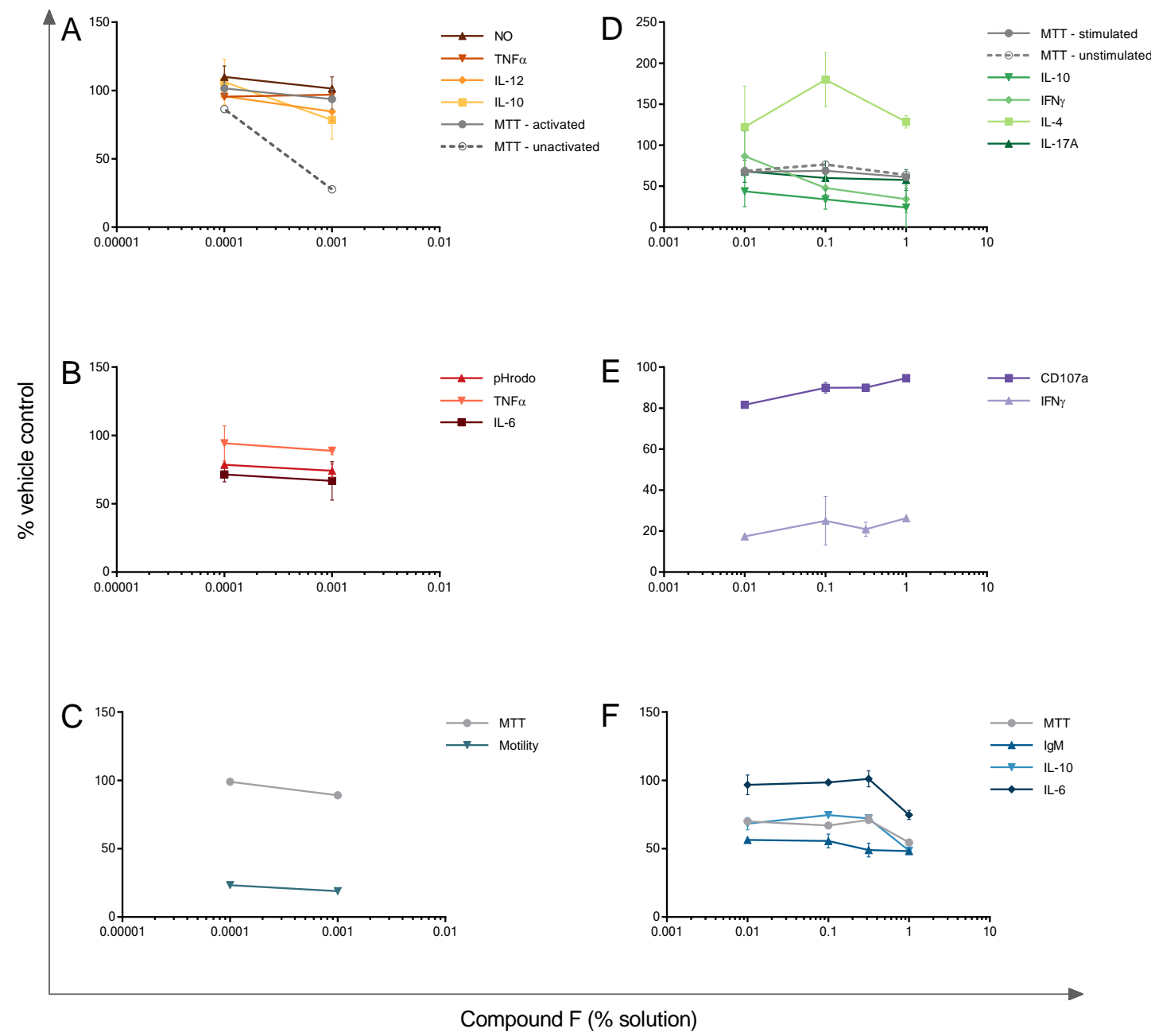

Figure 9.19. Screen outputs for Compound F

Compound F, at indicated concentrations, was run through the six assays. Macrophage activation (A), phagocytosis (B) and motility (C) are macrophage-based assays. In the right column are the splenocyte-based assays: helper T cell activation (D), CTL degranulation (E) and B cell activity (F). Experiments were conducted as explained in thesis methods (Ch 2). Shown are compound-induced changes compared to vehicle controls (i.e. \% vehicle). Closure in vehicle-treated wells during the motility assay averaged $74.0 \%$ (SD = 15.9\%). Data are means and SEM from each experiment which was conducted once in duplicate. 


\subsubsection{Compound G}

\subsubsection{Concentration selection for compound G}

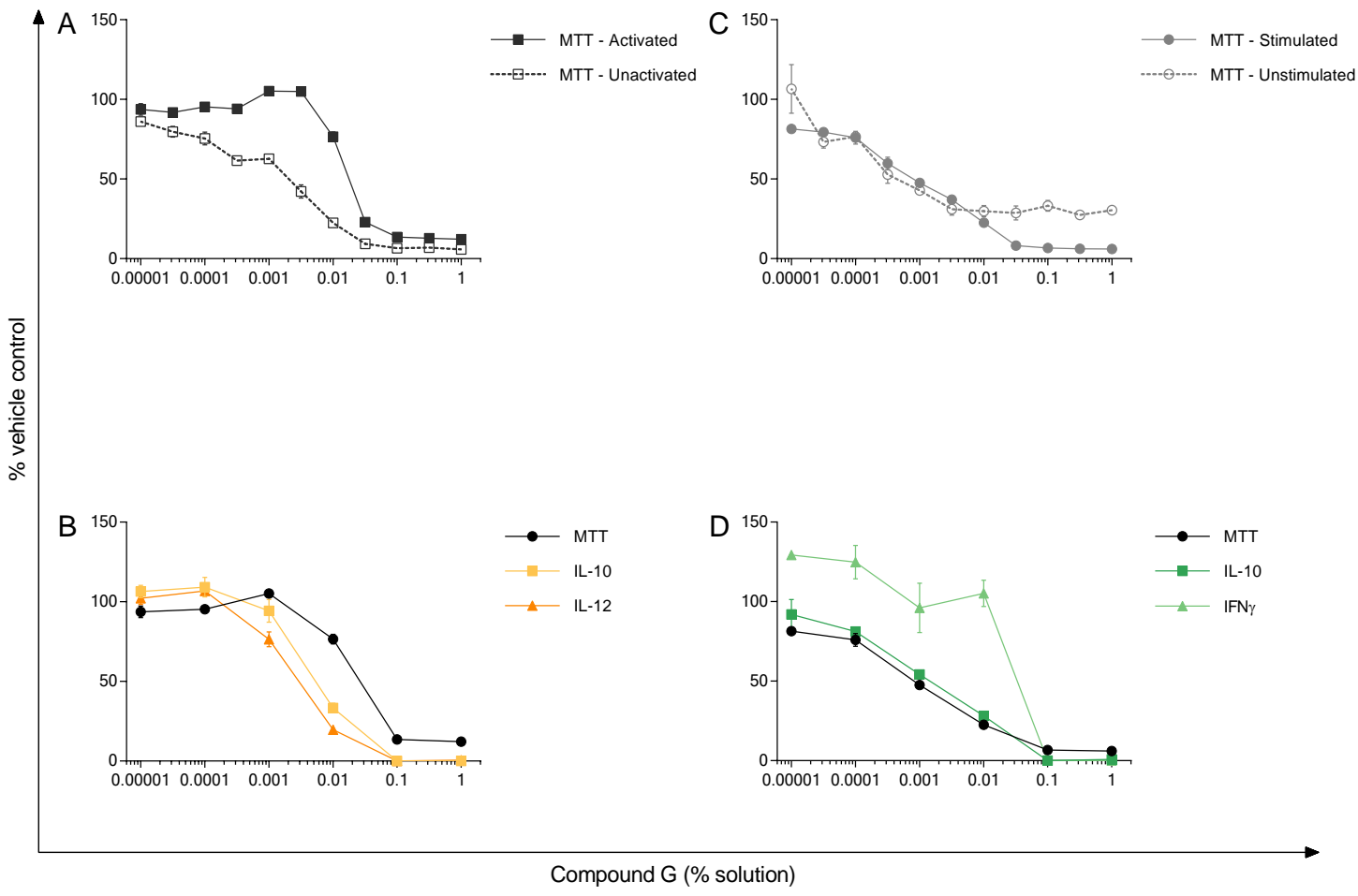

Figure 9.20. Concentration identification for compound G using stimulated macrophages and Th cells Activated macrophages were treated with Compound G and viability (A) and IL-10 and IL-12 (B) was assessed. Similarly, Helper T cells were activated in the presence of Compound G to generate MTT (C) and IL-10 and IFN $\gamma$ data (D). Data were used to select concentrations for further testing as described in (Ch 3, section 5.3.1)

Concentrations of compound $\mathrm{G}$ to use through the screening strategy were identified from macrophage activation and helper $\mathrm{T}$ cell activation assays.

Compound G showed cytotoxic effects on both cell types. The $\mathrm{IC}_{20}$ was seen with $0.009 \%$ solution for macrophages and $0.00017 \%$ for splenocytes. Additionally, $0.01 \%$ solution was included for its lack of toxicity on activated macrophages whilst being a high concentration that decreased cytokine production, and $0.001 \%$ was included for being a log-fold concentration below the $\mathrm{IC}_{20}$. At this concentration, unstimulated macrophages were still more sensitive to compound than activated counterparts, an effect seen at the $\mathrm{IC}_{20}$. For splenocytes, $0.001 \%$ solution was also selected for being a log-fold concentration close to the $\mathrm{IC}_{20}$. At the lower $0.0001 \%$ solution, which was also selected, the compound was nontoxic. 


\subsubsection{Screening data for Compound G}

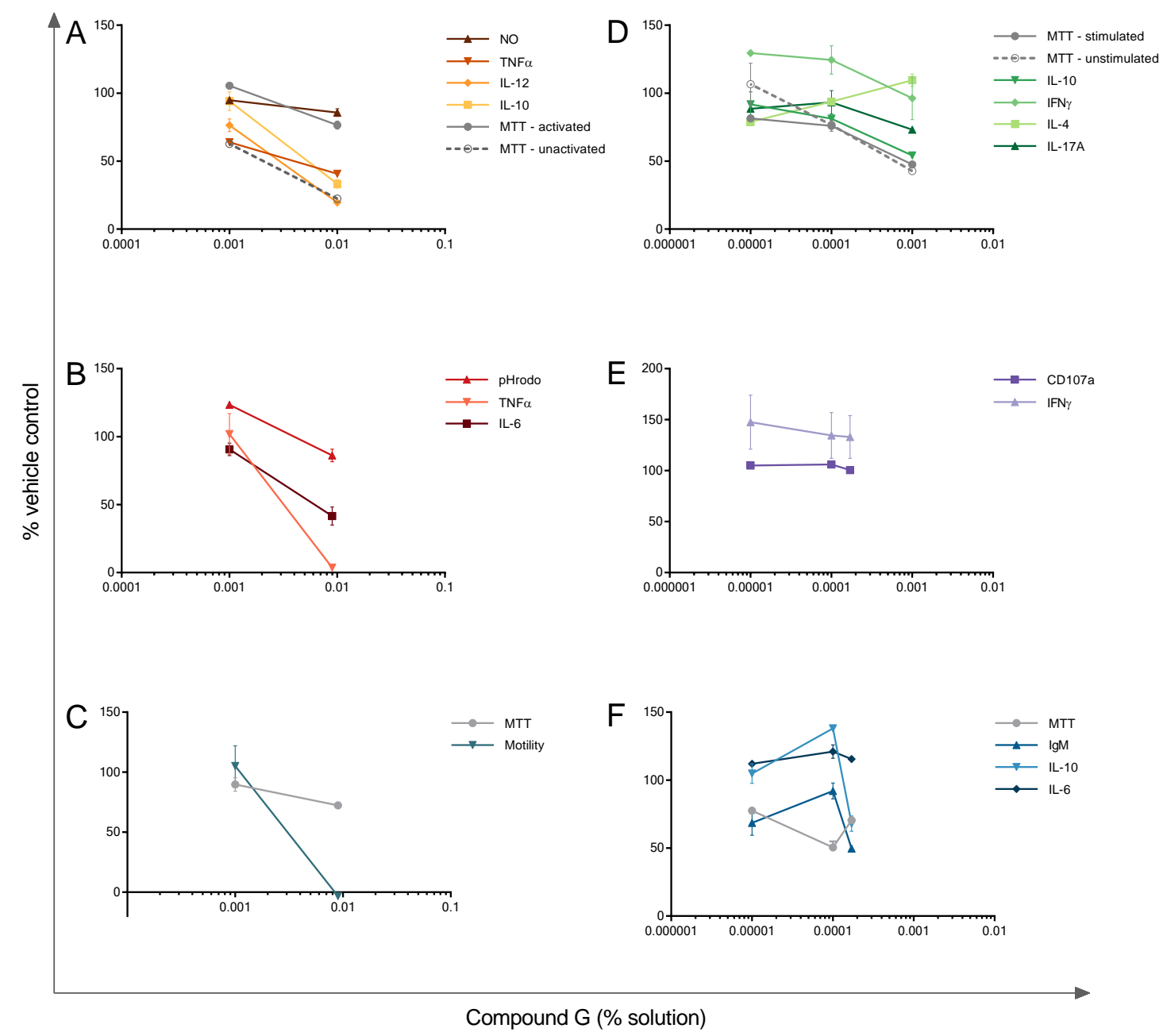

Figure 9.21. Screen outputs for Compound G

Compound G, at indicated concentrations, was run through the six assays. Macrophage activation (A), phagocytosis (B) and motility (C) are macrophage-based assays. In the right column are the splenocyte-based assays: helper T cell activation (D), CTL degranulation (E) and B cell activity (F). Experiments were conducted as explained in thesis methods (Ch 2). Shown are compound-induced changes compared to vehicle controls (i.e. \% vehicle). Closure in vehicle-treated wells during the motility assay averaged $74.0 \%$ (SD = 15.9\%). Data are means and SEM from each experiment which was conducted once in duplicate. 


\subsubsection{Compound $\mathrm{H}$}

\subsubsection{Concentration selection for compound $\mathrm{H}$}

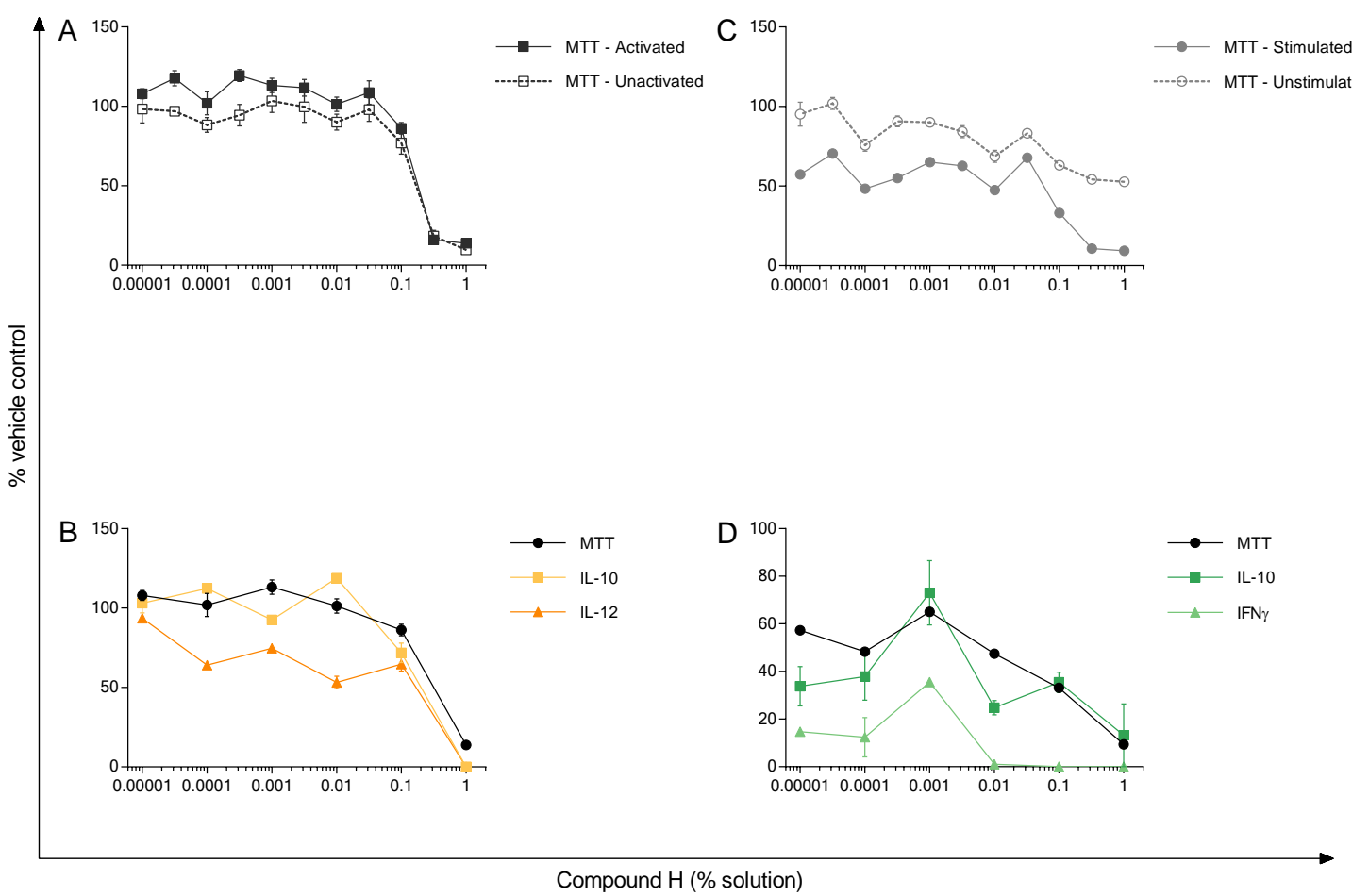

Figure 9.22. Concentration identification for compound $\mathbf{H}$ using stimulated macrophages and Th cells Activated macrophages were treated with Compound $\mathrm{H}$ and viability (A) and IL-10 and IL-12 (B) was assessed. Similarly, Helper T cells were activated in the presence of Compound H to generate MTT (C) and IL-10 and IFN $\gamma$ data (D). Data were used to select concentrations for further testing as described in (Ch 3, section 5.3.1)

Concentrations of compound $\mathrm{H}$ to use through the screening strategy were identified from macrophage activation and helper T cell activation assays.

Compound $\mathrm{H}$ was cytotoxic to macrophages. The $\mathrm{IC}_{20}$ was seen with $0.095 \%$ solution. 0.01\% was included for being non-toxic and exhibiting effects on macrophage cytokine production. The $\mathrm{IC}_{20}$ could not be calculated for splenocytes. Instead, 0.00001, 0.0001, 0.001 and $0.01 \%$ solutions were chosen as a difference in stimulated and unstimulated splenocyte viability is observed, and interesting changes to both IL-10 and IL-12 cytokine production by the stimulated Th cells. These changes fluctuated with different concentrations of compound $\mathrm{H}$. 


\subsubsection{Screening data for Compound $\mathrm{H}$}

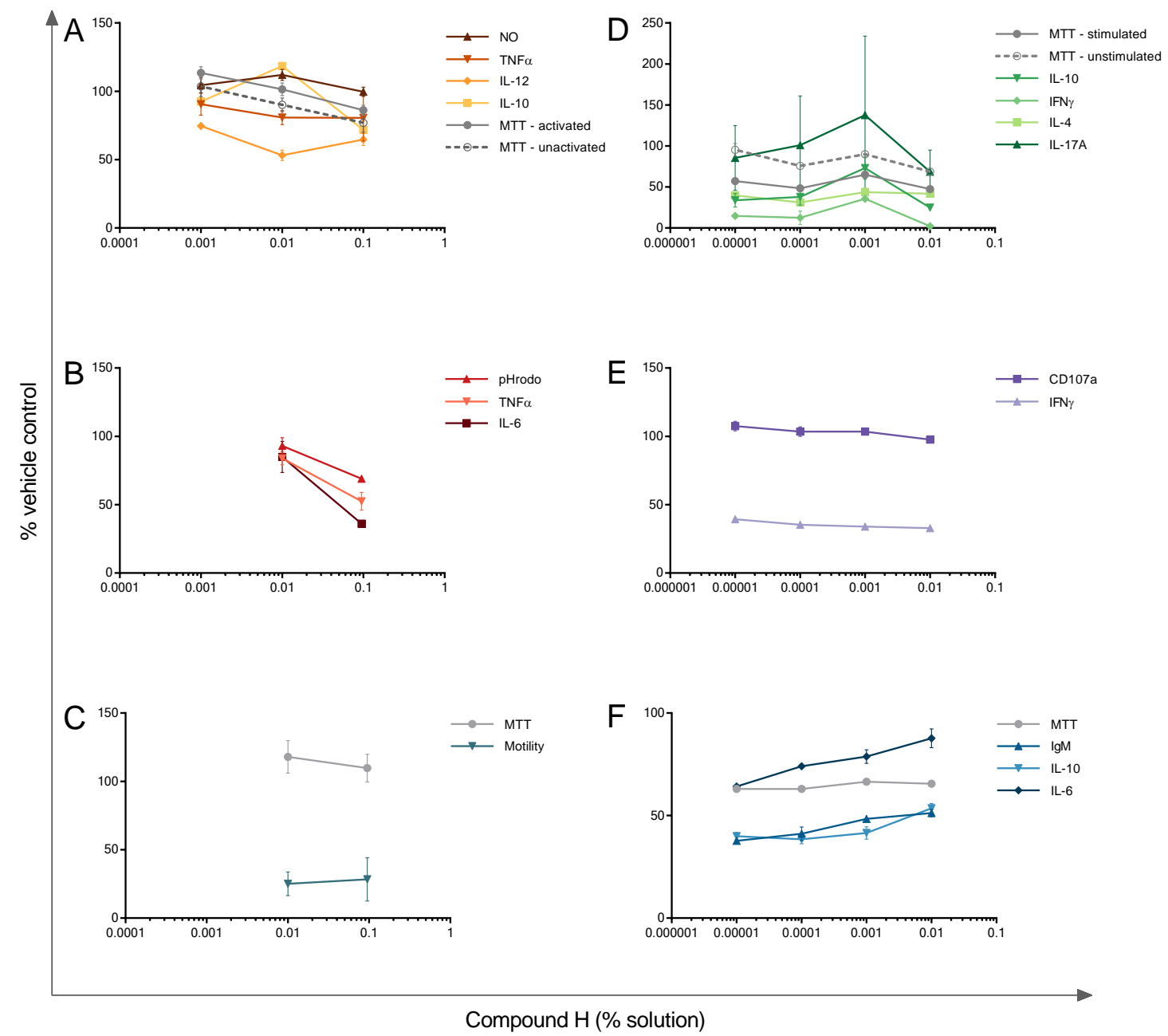

Figure 9.23. Screen outputs for Compound $H$

Compound $\mathrm{H}$, at indicated concentrations, was run through the six assays. Macrophage activation (A), phagocytosis (B) and motility (C) are macrophage-based assays. In the right column are the splenocyte-based assays: helper T cell activation (D), CTL degranulation (E) and B cell activity (F). Experiments were conducted as explained in thesis methods (Ch 2). Shown are compound-induced changes compared to vehicle controls (i.e. \% vehicle). Closure in vehicle-treated wells during the motility assay averaged $74.0 \%$ (SD = 15.9\%). Data are means and SEM from each experiment which was conducted once in duplicate. 


\subsubsection{Compound I}

\subsubsection{Concentration selection for compound I}

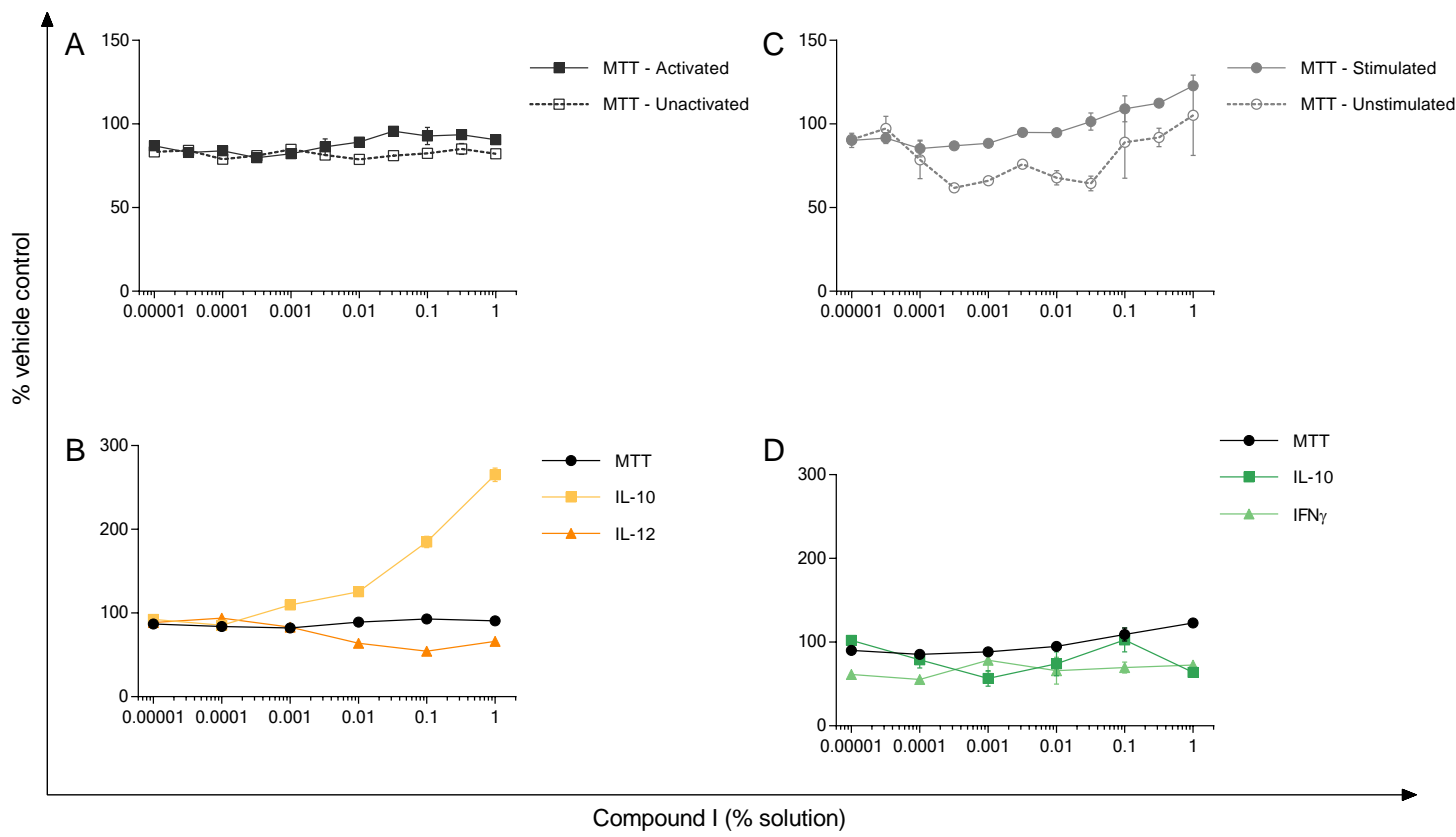

Figure 9.24. Concentration identification for compound I using stimulated macrophages and Th cells Activated macrophages were treated with Compound I and viability (A) and IL-10 and IL-12 (B) was assessed. Similarly, Helper T cells were activated in the presence of Compound I to generate MTT (C) and IL-10 and IFN $\gamma$ data (D). Data were used to select concentrations for further testing as described in (Ch 3, section 5.3.1)

Concentrations of compound I to use through the screening strategy were identified from macrophage activation and helper T cell activation assays.

Compound I was not toxic to either cell type. Macrophage concentrations (0.01, 0.1 and $1 \%$ ) were selected as these concentrations altered cytokine production. Concentrations of $0.01 \%, 0.1 \%$ and $1 \%$ were selected for splenocyte assays as these concentrations altered IL-10 and IFN $\gamma$ production. Though IL-10 exhibited concentration-dependent changes, these were also reflected in the selected concentrations. 


\subsubsection{Screening data for Compound I}

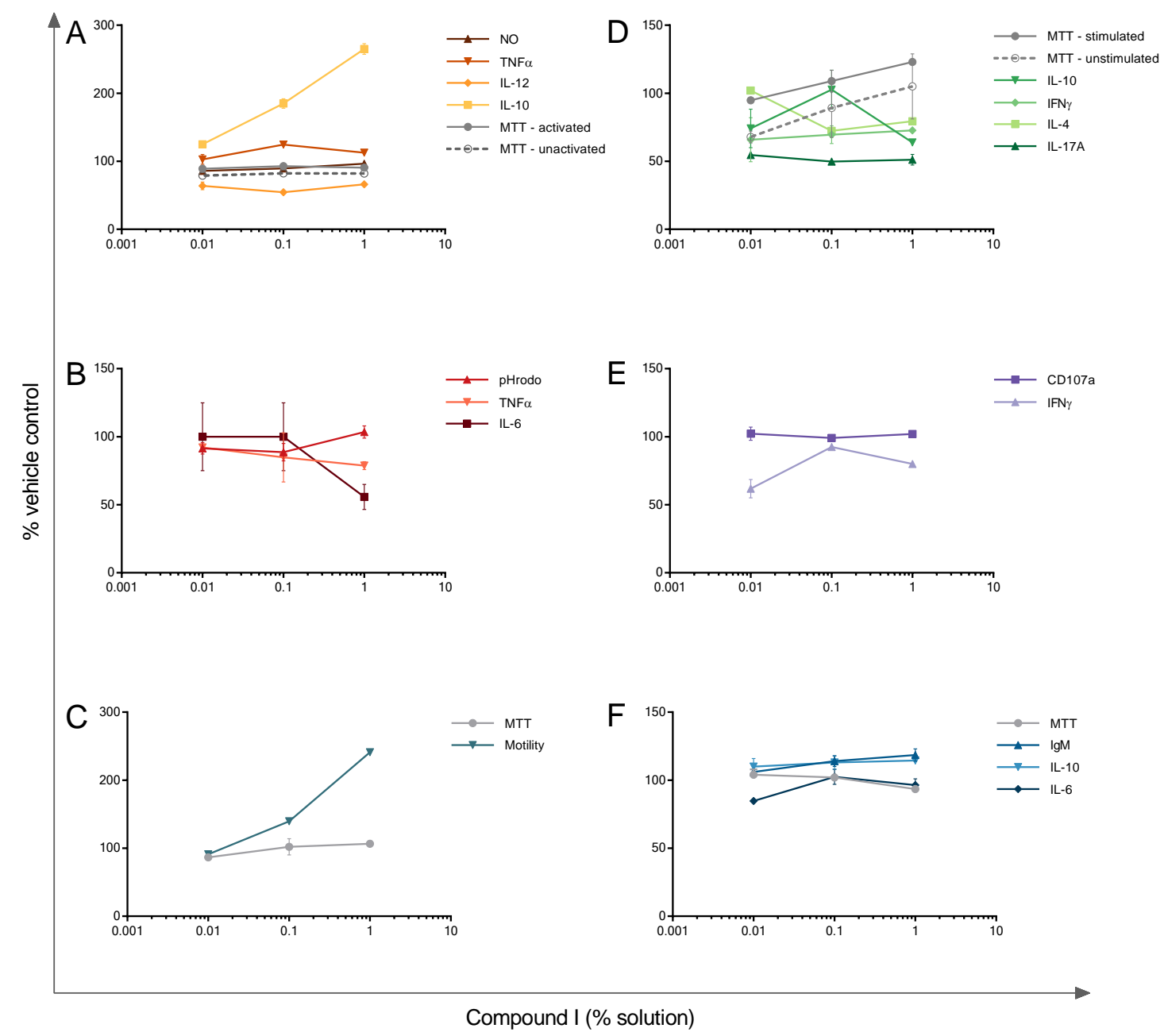

Figure 9.25. Screen outputs for Compound I

Compound I, at indicated concentrations, was run through the six assays. Macrophage activation (A), phagocytosis (B) and motility (C) are macrophage-based assays. In the right column are the splenocyte-based assays: helper T cell activation (D), CTL degranulation (E) and B cell activity (F). Experiments were conducted as explained in thesis methods (Ch 2). Shown are compound-induced changes compared to vehicle controls (i.e. \% vehicle). Closure in vehicle-treated wells during the motility assay averaged $74.0 \%$ (SD = 15.9\%). Data are means and SEM from each experiment which was conducted once in duplicate. 


\subsubsection{Compound J}

\subsubsection{Concentration selection for compound J}

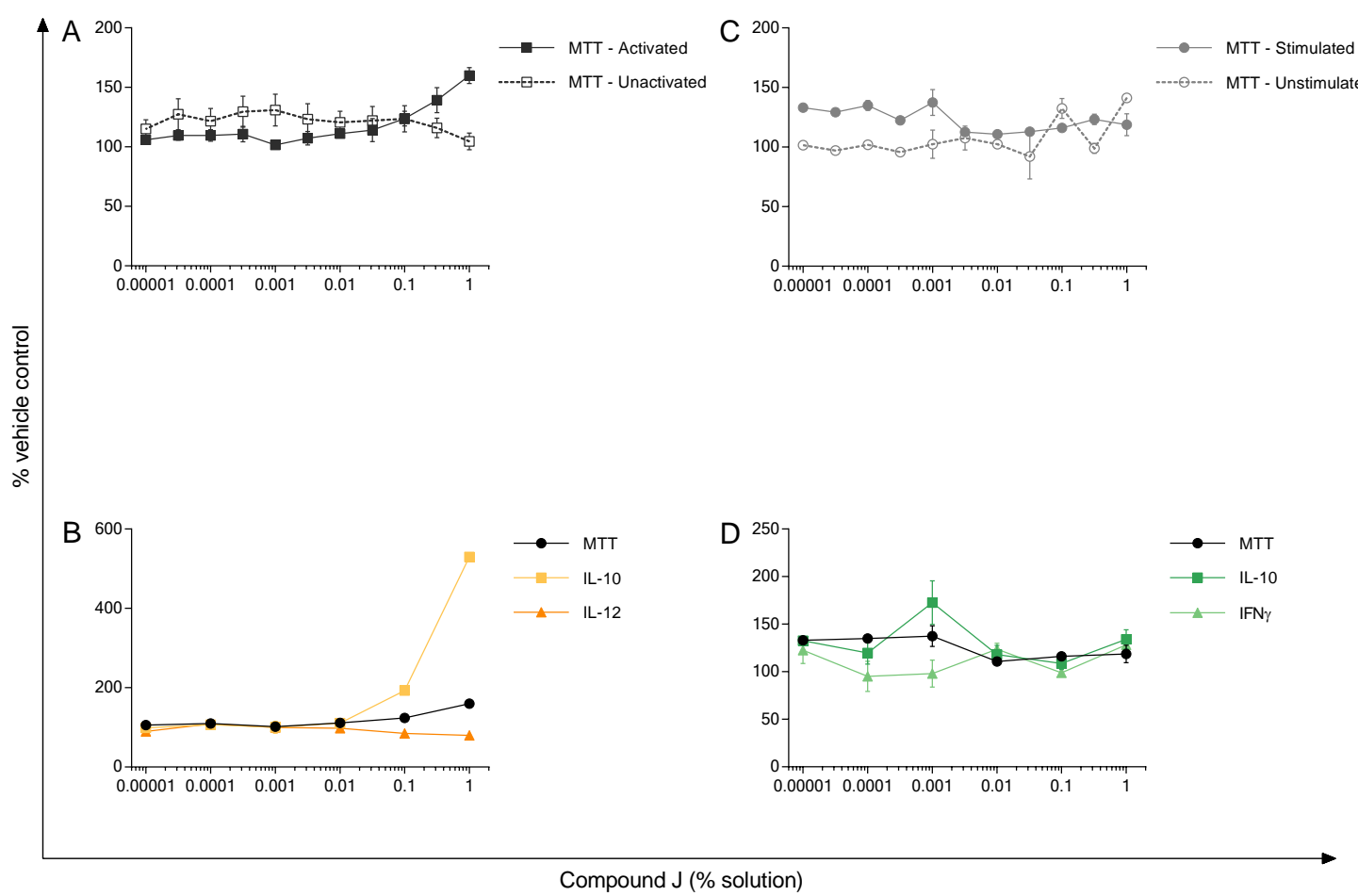

Figure 9.26. Concentration identification for compound $\mathbf{J}$ using stimulated macrophages and Th cells Activated macrophages were treated with Compound J and viability (A) and IL-10 and IL-12 (B) was assessed. Similarly, Helper T cells were activated in the presence of Compound J to generate MTT (C) and IL-10 and IFN $\gamma$ data (D). Data were used to select concentrations for further testing as described in (Ch 3, section 5.3.1)

Concentrations of compound $\mathrm{J}$ to use through the screening strategy were identified from macrophage activation and helper $\mathrm{T}$ cell activation assays.

Compound J was also non-toxic to both cell types. Much like concentration selection for the previous compound, $0.01,0.1$ and $1 \%$ solutions were selected for macrophage assays due to their effects on cytokine production. For splenocytes, 0.001, 0.01, 0.1 and 1\% solutions were selected. The highest three show non-toxicity, and $0.001 \%$ solution caused IFN $\gamma$ production to decrease, and IL-10 production to increase in activated Th cells. 


\subsubsection{Screening data for Compound J}

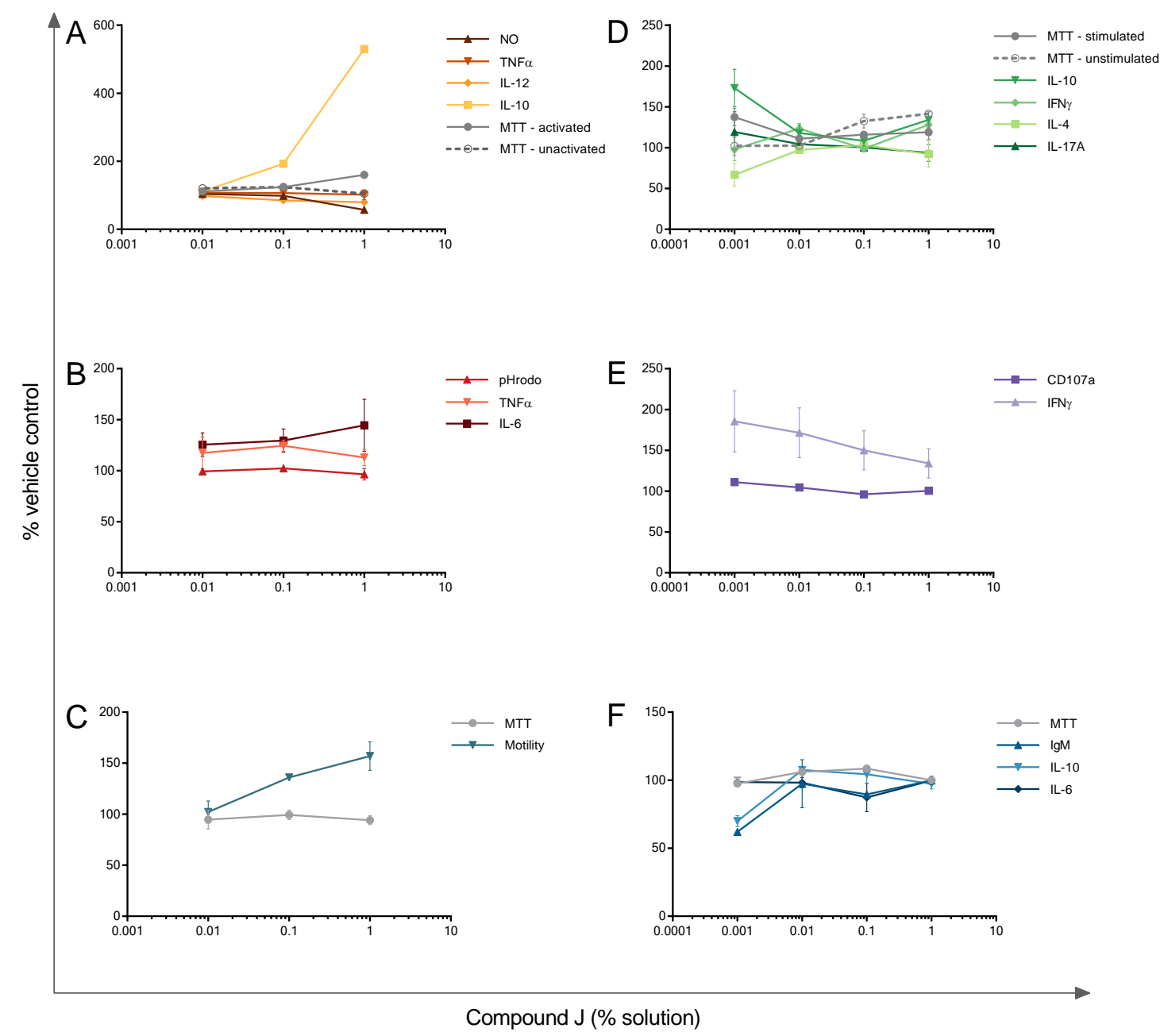

Figure 9.27. Screen outputs for Compound $\mathbf{J}$

Compound $\mathrm{J}$, at indicated concentrations, was run through the six assays. Macrophage activation (A), phagocytosis (B) and motility (C) are macrophage-based assays. In the right column are the splenocyte-based assays: helper T cell activation (D), CTL degranulation (E) and B cell activity (F). Experiments were conducted as explained in thesis methods (Ch 2). Shown are compound-induced changes compared to vehicle controls (i.e. \% vehicle). Closure in vehicle-treated wells during the motility assay averaged $74.0 \%$ (SD = 15.9\%). Data are means and SEM from each experiment which was conducted once in duplicate. 


\subsubsection{Compound $\mathrm{K}$}

\subsubsection{Concentration selection for compound $\mathrm{K}$}

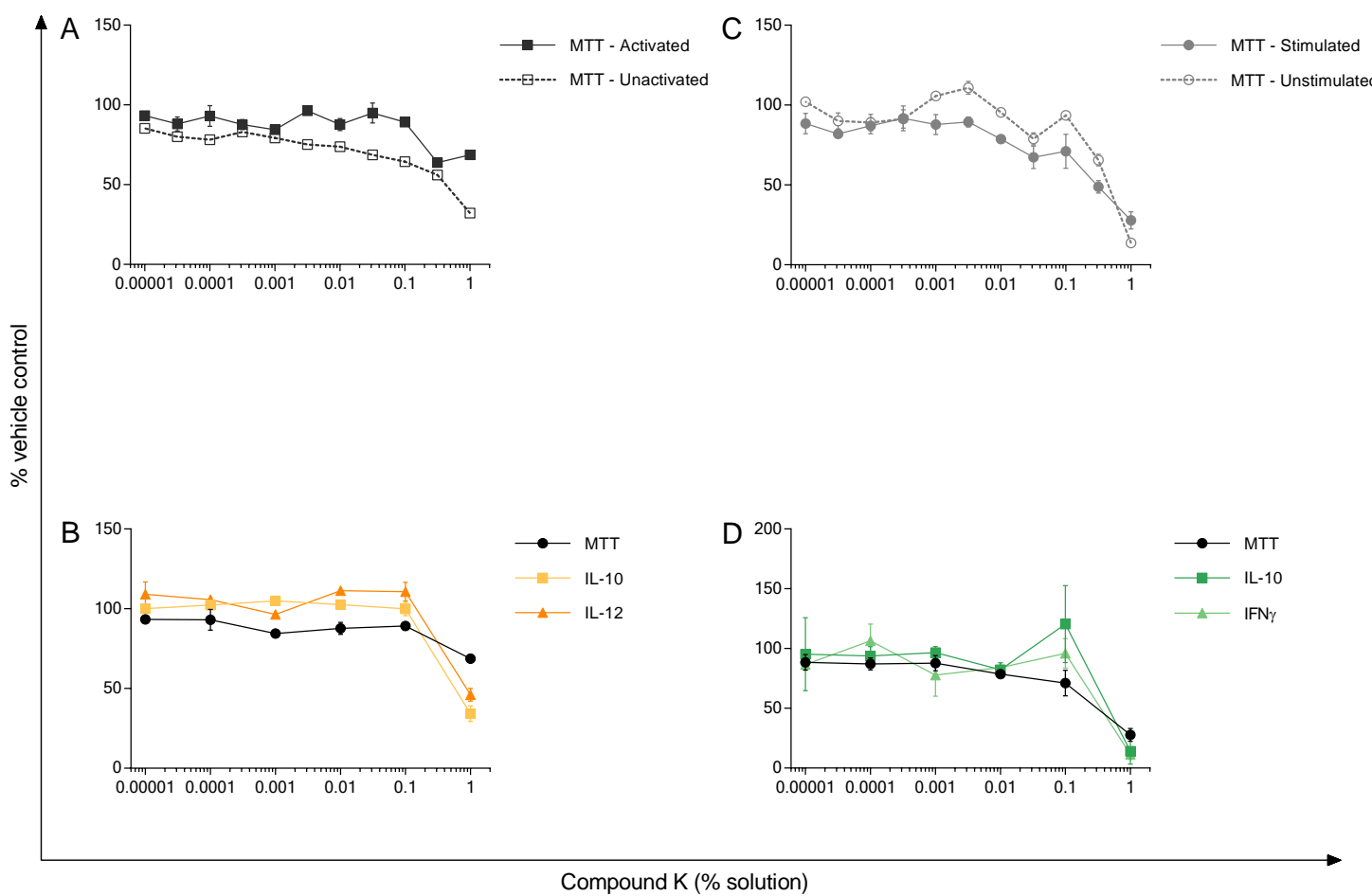

Figure 9.28. Concentration identification for compound $\mathrm{K}$ using stimulated macrophages and Th cells Activated macrophages were treated with Compound K and viability (A) and IL-10 and IL-12 (B) was assessed. Similarly, Helper T cells were activated in the presence of Compound K to generate MTT (C) and IL-10 and IFN $\gamma$ data (D). Data were used to select concentrations for further testing as described in (Ch 3, section 5.3.1)

Concentrations of compound $\mathrm{K}$ to use through the screening strategy were identified from macrophage activation and helper T cell activation assays.

Toxicity identified the highest tolerated concentrations of compound $\mathrm{K}$ for both macrophages and splenocytes. Three high concentrations were selected for macrophages (0.001, 0.01 and $0.1 \%)$ and splenocytes $(0.001,0.01,0.1 \%)$. For both the cell types the concentrations were the same by chance. 


\subsubsection{Screening data for Compound K}

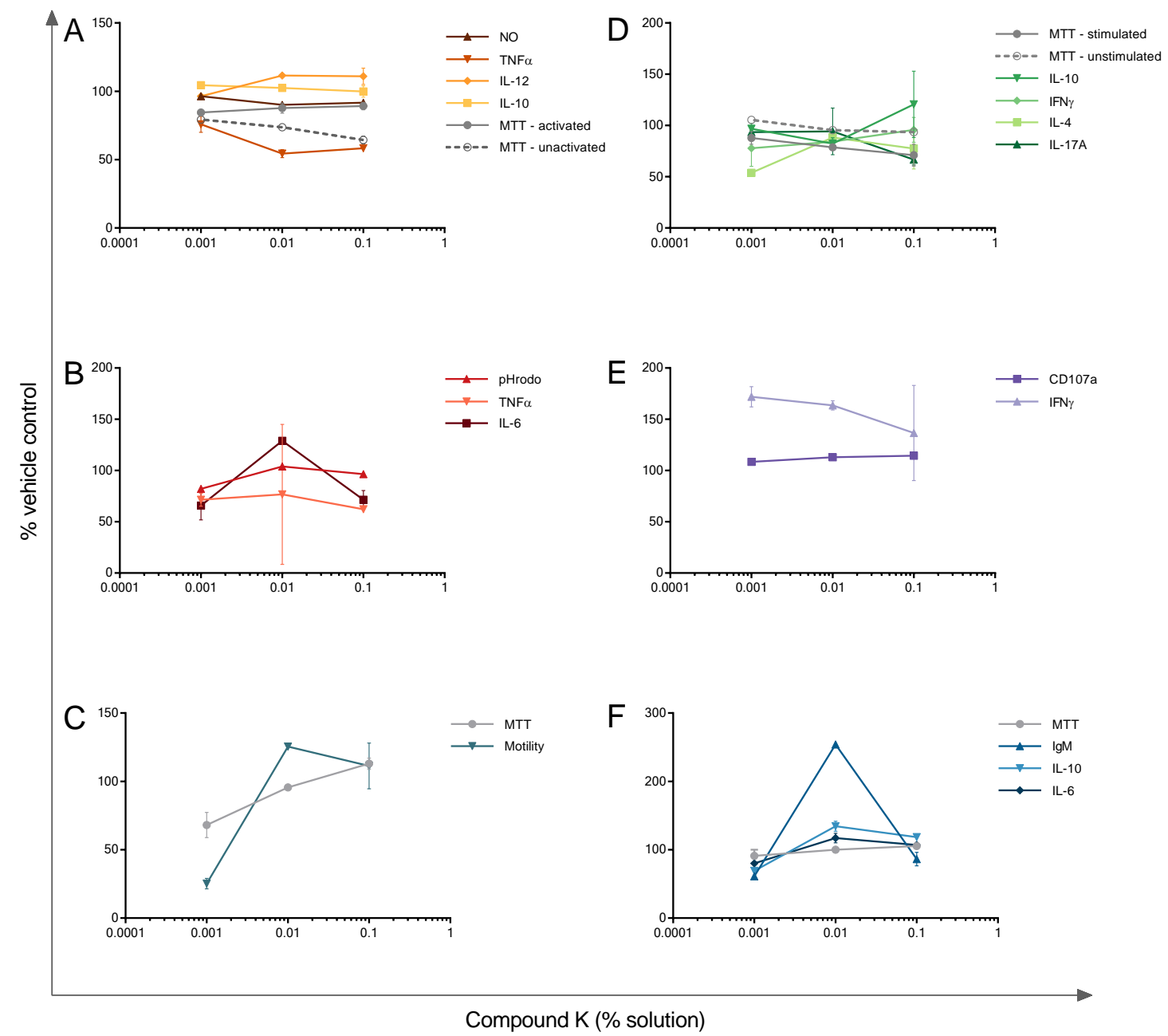

Figure 9.29. Screen outputs for Compound $K$

Compound $\mathrm{K}$, at indicated concentrations, was run through the six assays. Macrophage activation (A), phagocytosis (B) and motility (C) are macrophage-based assays. In the right column are the splenocyte-based assays: helper T cell activation (D), CTL degranulation (E) and B cell activity (F). Experiments were conducted as explained in thesis methods (Ch 2). Shown are compound-induced changes compared to vehicle controls (i.e. \% vehicle). Closure in vehicle-treated wells during the motility assay averaged $74.0 \%$ (SD = 15.9\%). Data are means and SEM from each experiment which was conducted once in duplicate. 


\subsubsection{Compound L}

\subsubsection{Concentration selection for compound $\mathrm{L}$}

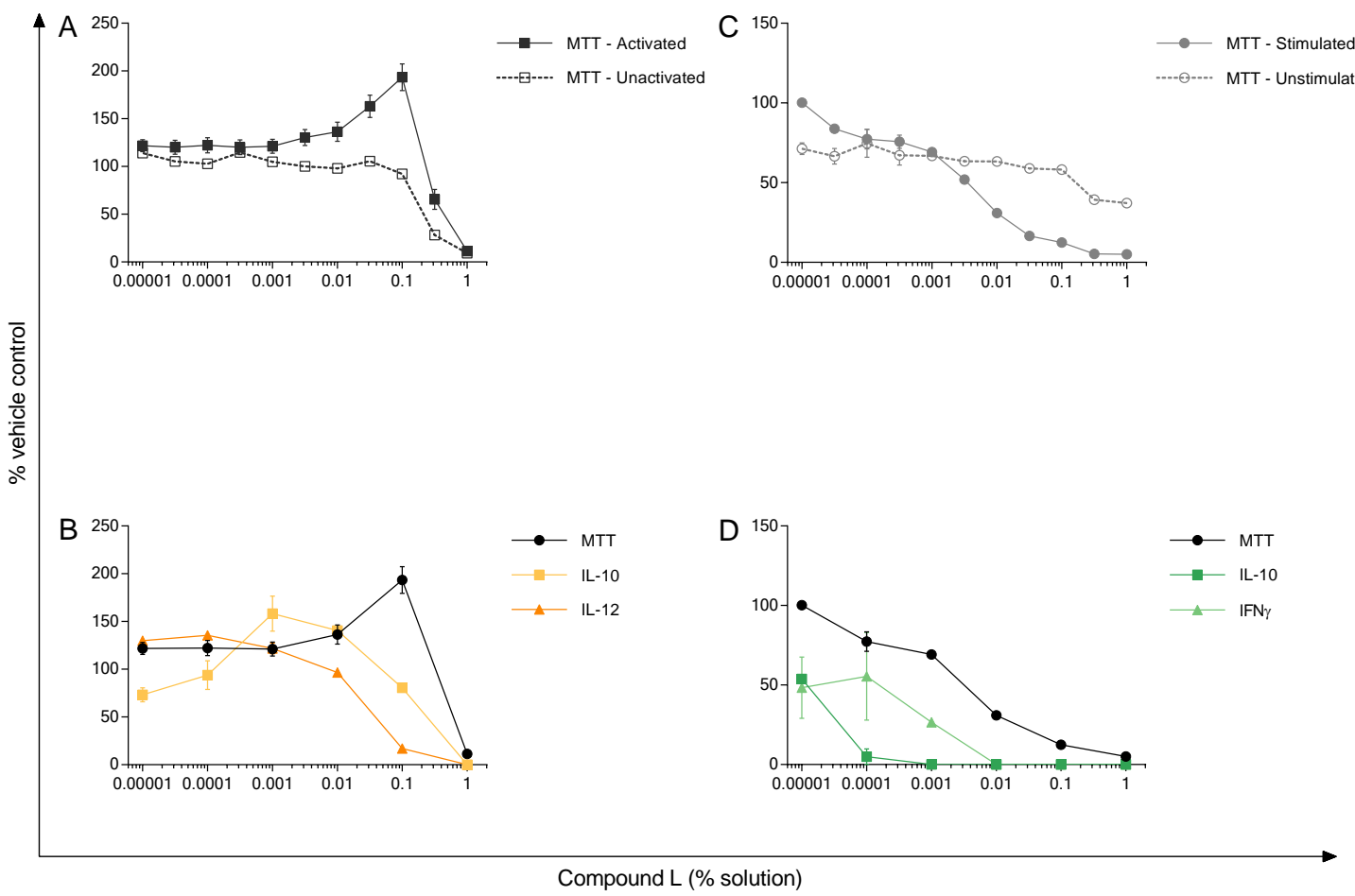

Figure 9.30. Concentration identification for compound $L$ using stimulated macrophages and Th cells Activated macrophages were treated with Compound L and viability (A) and IL-10 and IL-12 (B) was assessed. Similarly, Helper T cells were activated in the presence of Compound L to generate MTT (C) and IL-10 and IFN $\gamma$ data (D). Data were used to select concentrations for further testing as described in (Ch 3, section 5.3.1)

Concentrations of compound $\mathrm{L}$ to use through the screening strategy were identified from macrophage activation and helper $\mathrm{T}$ cell activation assays.

Cytotoxicity also guided compound L concentration selection. For macrophages, high, non-toxic concentrations were $0.001,0.01$ and $0.1 \%$ solutions. Interestingly, at the higher concentration, cytokine production is altered. For splenocytes, the highest non-toxic concentrations were $0.00001,0.0001$ and $0.001 \%$ solutions. These concentrations also caused IFN $\gamma$ and IL-10 production to decrease. 


\subsubsection{Screening data for Compound L}

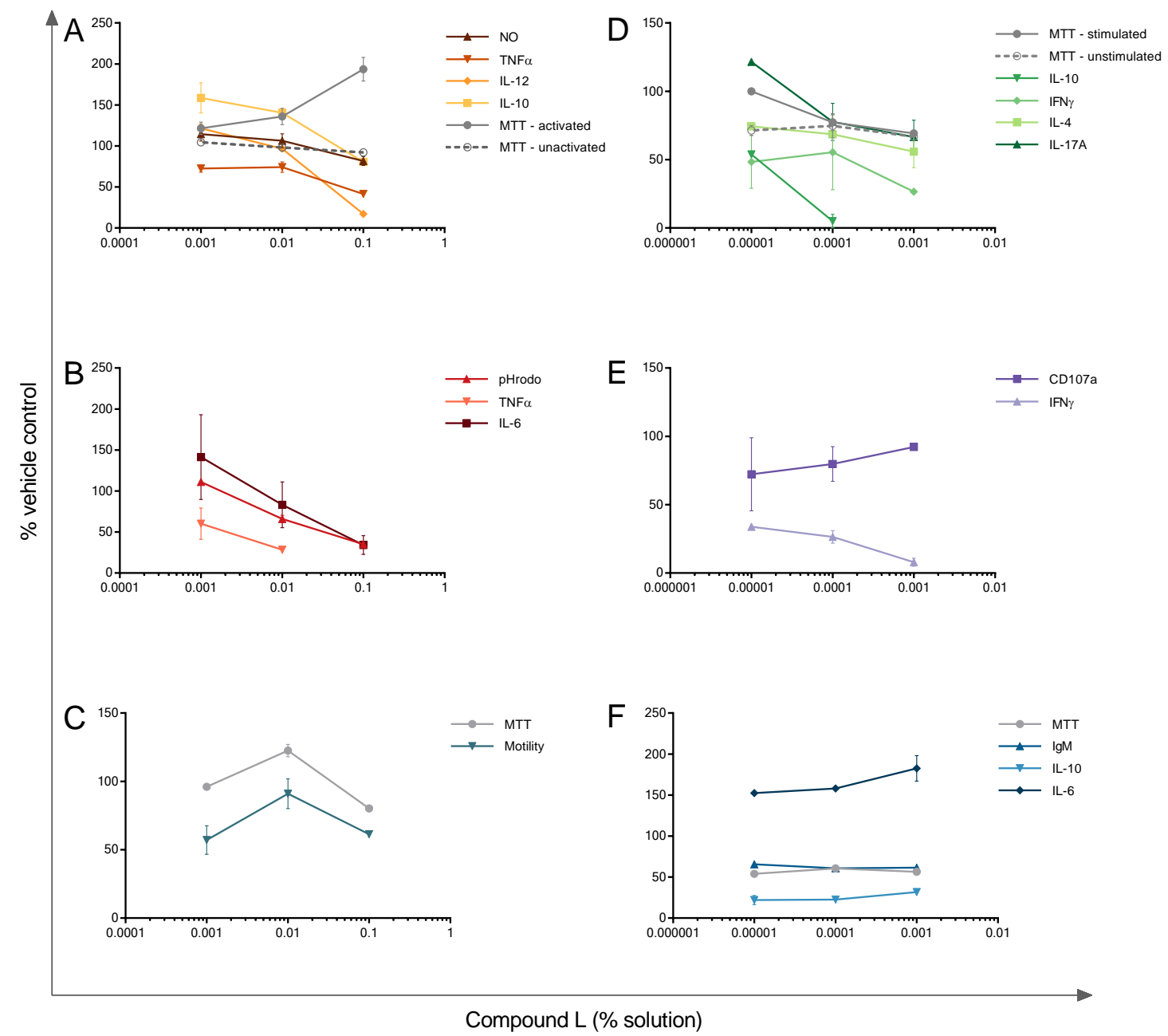

Figure 9.31. Screen outputs for Compound $L$

Compound L, at indicated concentrations, was run through the six assays. Macrophage activation (A), phagocytosis (B) and motility (C) are macrophage-based assays. In the right column are the splenocyte-based assays: helper T cell activation (D), CTL degranulation (E) and B cell activity (F). Experiments were conducted as explained in thesis methods (Ch 2). Shown are compound-induced changes compared to vehicle controls (i.e. \% vehicle). Closure in vehicle-treated wells during the motility assay averaged $74.0 \%$ (SD = 15.9\%). Data are means and SEM from each experiment which was conducted once in duplicate. 


\subsection{Supplementary material for Chapter 6}

\subsubsection{P. insularum (aqueous extract)}

\subsubsection{Screening data for P. insularum (aqueous extract)}

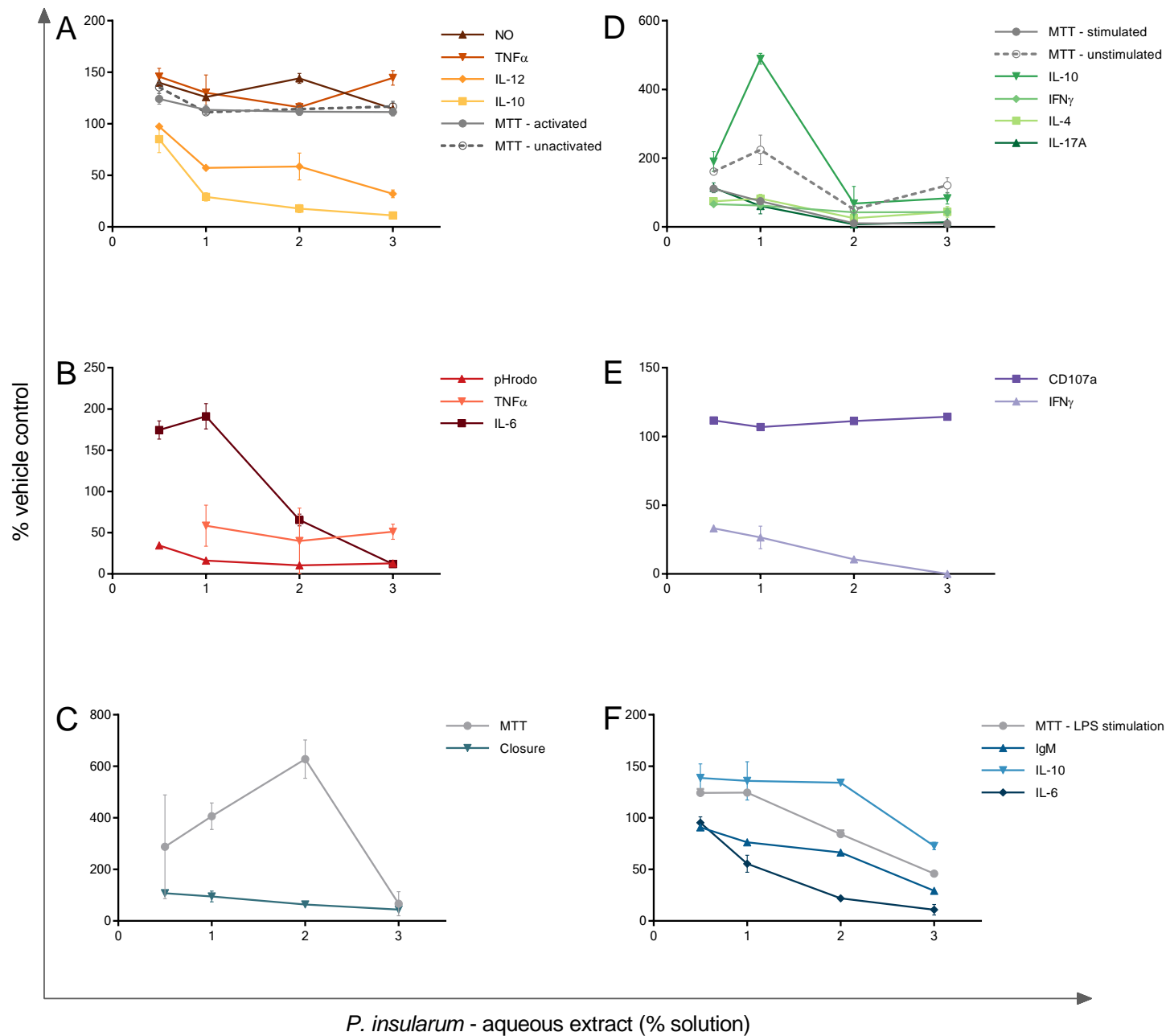

Figure 9.32. Screen outputs for $P$. insularum - aqueous extract

$P$. insularum (aqueous extract) was run through the six assays at the indicated concentrations. Macrophage activation (A), phagocytosis (B) and motility (C) are macrophage-based assays. In the right column are the splenocyte-based assays: helper T cell activation (D), CTL degranulation (E) and B cell activity (F). Experiments were conducted as explained in thesis methods (Ch 2). Shown are compound-induced changes compared to vehicle controls (i.e. \% vehicle). Closure in vehicle-treated wells during the motility assay averaged $74.0 \%(S D=15.9 \%)$. Data are means and SEM from each experiment which was conducted once in duplicate. 


\subsubsection{P. insularum (methanolic extract)}

\subsubsection{Concentration selection for P. insularum (methanolic extract)}

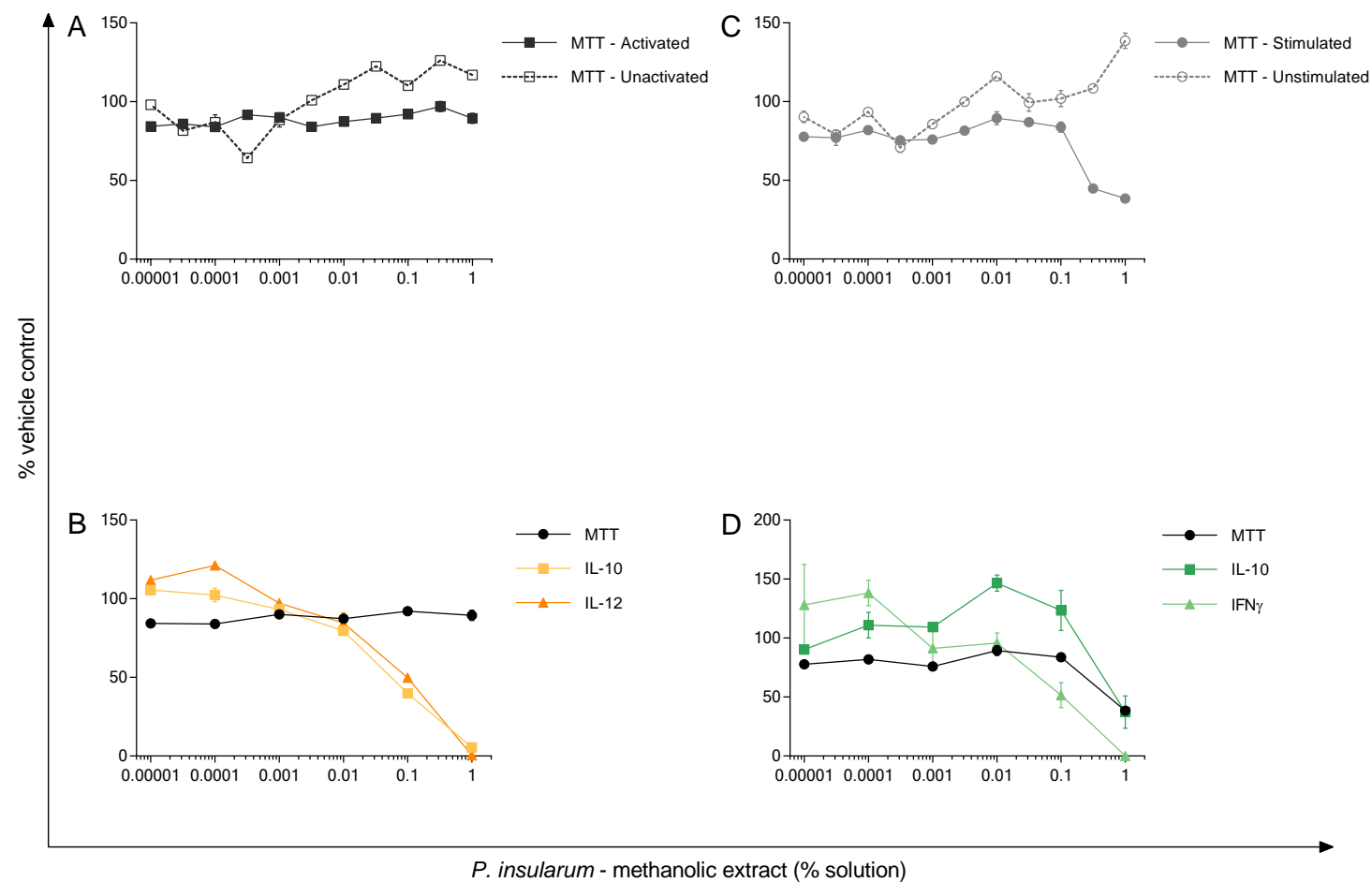

Figure 9.33. Concentration identification for the methanolic extract of $P$. insularum using stimulated macrophages and Th cells

Activated macrophages were treated with the methanolic extract of $P$. insularum and viability (A) and IL-10 and IL-12 (B) was assessed. Similarly, Helper T cells were activated in the presence of the methanolic extract of $P$. insularum to generate MTT (C) and IL-10 and IFN $\gamma$ data (D). Data were used to select concentrations for further testing as described in (Ch 3 , section 5.3.1)

Due to the lack of cytotoxicity of methanolic $P$. insularum on macrophages and unactivated splenocytes, three high concentrations of extract were selected to test $(0.01,0.1$ and $1 \%)$ for both cell types. Interestingly, changes to macrophages cytokine production were observed, as well as decreased IFN $\gamma$ and increased IL-10 in stimulated Th cells. 


\subsubsection{Screening data for P. insularum (methanolic extract)}

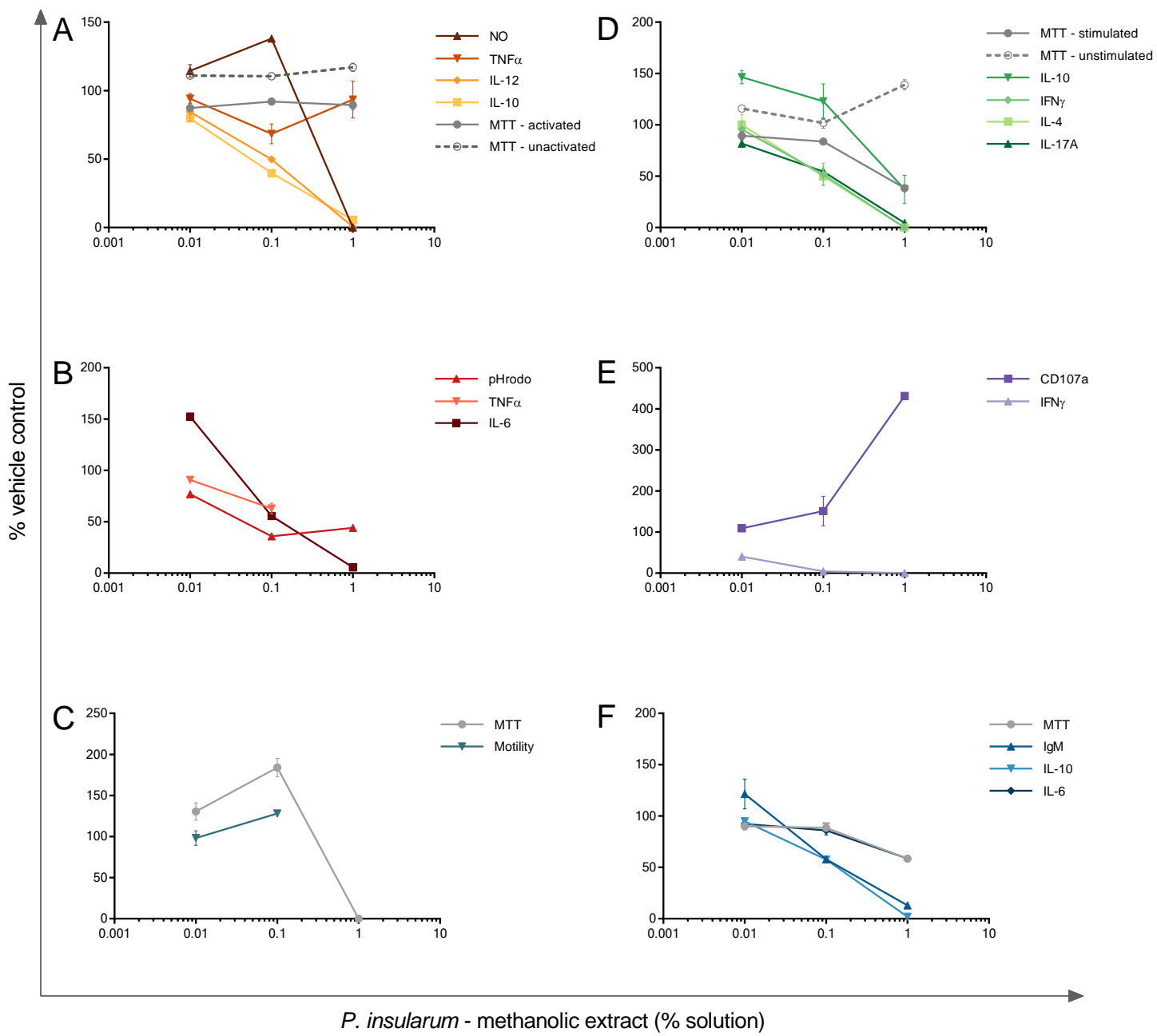

Figure 9.34. Screen outputs for $P$. insularum (methanolic extract)

$P$. insularum (methanolic extract) was run through the six assays at the indicated concentrations. Macrophage activation (A), phagocytosis (B) and motility (C) are macrophage-based assays. In the right column are the splenocyte-based assays: helper T cell activation (D), CTL degranulation (E) and B cell activity (F). Experiments were conducted as explained in thesis methods (Ch 2). Shown are compound-induced changes compared to vehicle controls (i.e. \% vehicle). Closure in vehicle-treated wells during the motility assay averaged 74.0\% (SD = 15.9\%). Data are means and SEM from each experiment which was conducted once in duplicate. 


\subsubsection{MTDIA}

\subsubsection{Concentration selection for MTDIA}

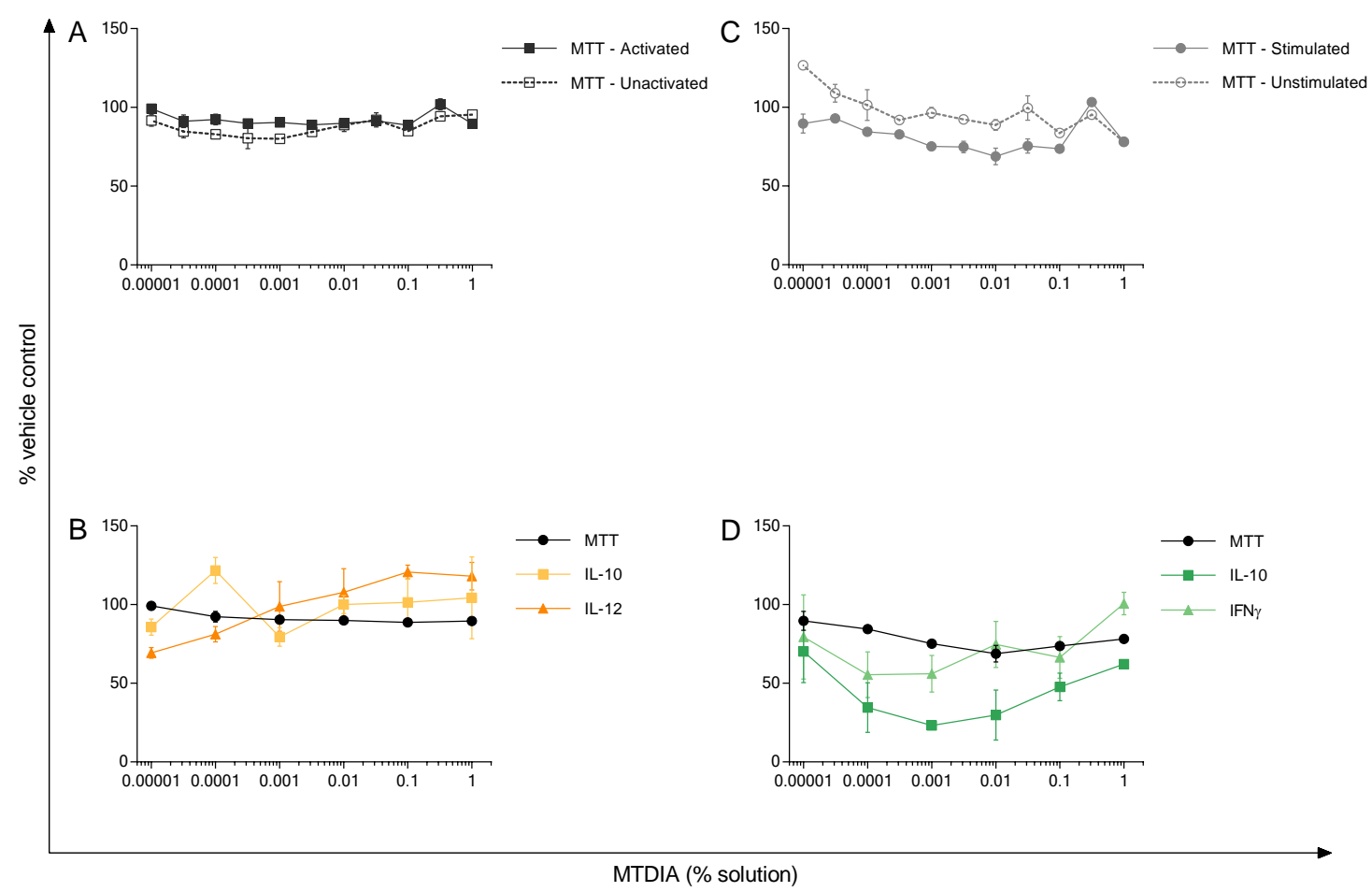

Figure 9.35. Concentration identification for MTDIA using stimulated macrophages and Th cells Activated macrophages were treated with MTDIA and viability (A) and IL-10 and IL-12 (B) was assessed. Similarly, Helper T cells were activated in the presence of MTDIA to generate MTT (C) and IL-10 and IFN $\gamma$ data (D). Data were used to select concentrations for further testing as described in (Ch 3, section 5.3.1)

MTDIA was also not toxic to the two cell types. For macrophages, $0.01,0.1$ and $1 \%$ solutions were selected for their changes to cytokine production (both increased). $0.0001 \%$ was included due to the different changes to cytokine production observed (moved in opposite directions). For splenocytes, four high concentrations were chosen $(0.001,0.01$, 0.1 and 1\%). These concentrations decreased IL-10 production and covered the subtle concentration-dependent changes to IFN $\gamma$ production by stimulated Th cells. 


\subsubsection{Screening data for MTDIA}

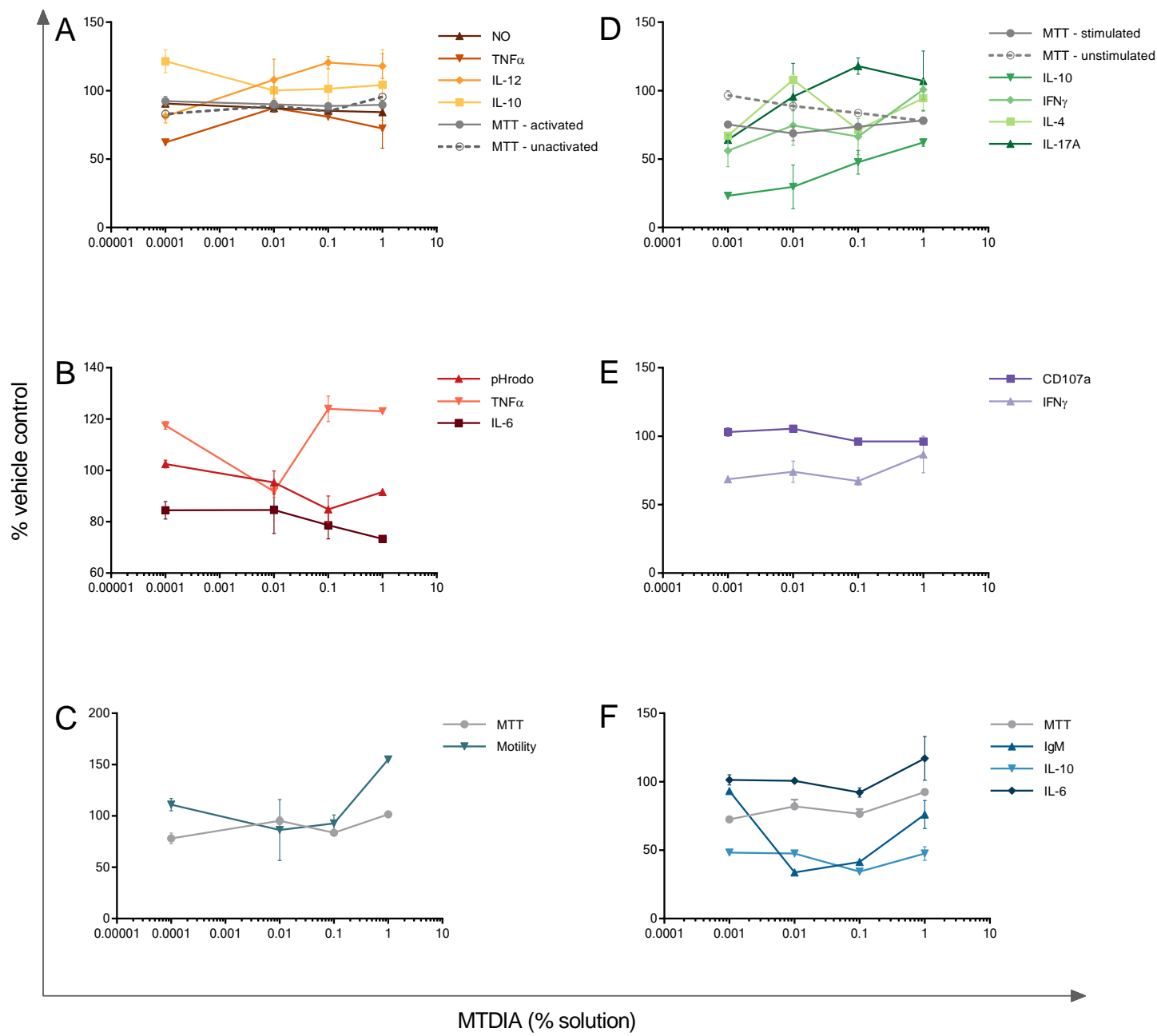

Figure 9.36. Screen outputs for MTDIA

MTDIA, at indicated concentrations, was run through the six assays. Macrophage activation (A), phagocytosis (B) and motility (C) are macrophage-based assays. In the right column are the splenocyte-based assays: helper T cell activation (D), CTL degranulation (E) and B cell activity (F). Experiments were conducted as explained in thesis methods (Ch 2). Shown are compound-induced changes compared to vehicle controls (i.e. \% vehicle). Closure in vehicle-treated wells during the motility assay averaged $74.0 \%$ (SD = 15.9\%). Data are means and SEM from each experiment which was conducted once in duplicate. 


\subsubsection{RAW cell viability with MTA and MTDIA treatment}
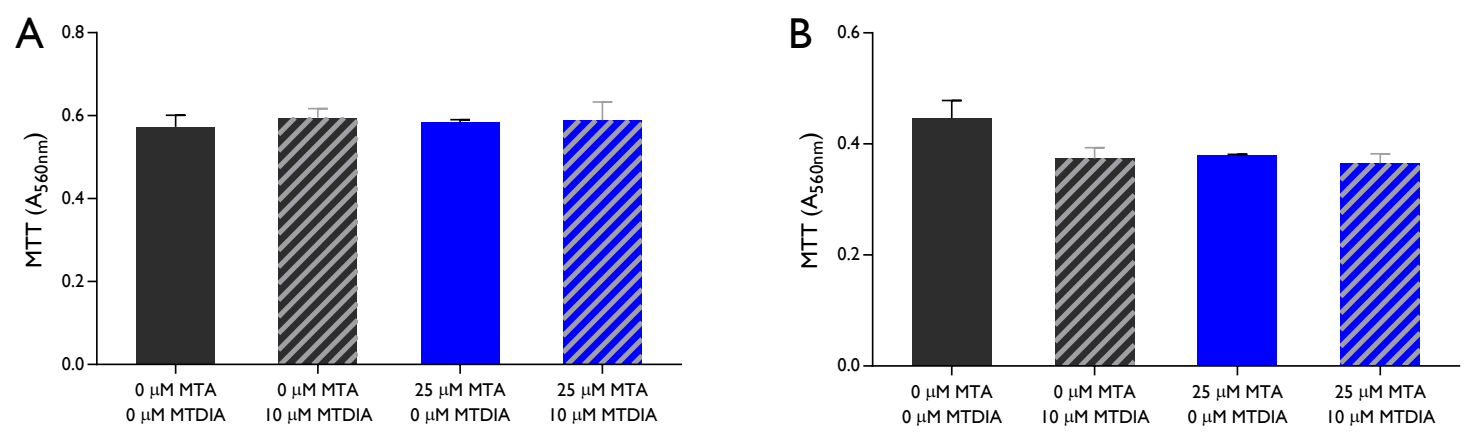

Figure 9.37. MTT of RAW264.7 cells is unaffected by MTA and MTDIA

50,000 RAW 264.7 cells were seeded into wells of a 96-well plate. After $18 \mathrm{~h}$, indicated concentrations of MTA and MTDIA were added to wells and cells were incubated for $24 \mathrm{~h}$; this is an unactivated macrophage condition (A). Alternatively, cells were primed with $20 \mathrm{U} / \mathrm{mL}$ IFN $\gamma$ for $18 \mathrm{~h}$ after plating. Indicated concentrations of MTA and MTDIA were added to wells, alongside $200 \mathrm{ng} / \mathrm{mL}$ LPS to induce activation (B). After $24 \mathrm{~h}$, cell viability was assessed by MTT. Solid black bars indicate untreated (vehicle) controls. Blue indicates the addition of $25 \mu \mathrm{M}$ MTA. Striped bars indicate the addition of $10 \mu \mathrm{M}$ MTDIA.

The addition of MTA and or MTDIA did not alter unactivated RAW 264.7 cell viability (Figure 9.37, A). Compared to untreated (vehicle) controls, cell viability was $104 \%$ with MTDIA treatment, 102\% with MTA treatment and 103\% with both added.

For activated macrophages (Figure 9.37, B), viability reduced slightly with treatments. Compared to untreated (vehicle) control, MTDIA reduced viability to 84\%, MTA reduced viability to $85 \%$ and when both were added, viability was $82 \%$ of vehicle control. 


\subsubsection{HS16-38}

\subsubsection{Concentration selection for compound HS16-38}

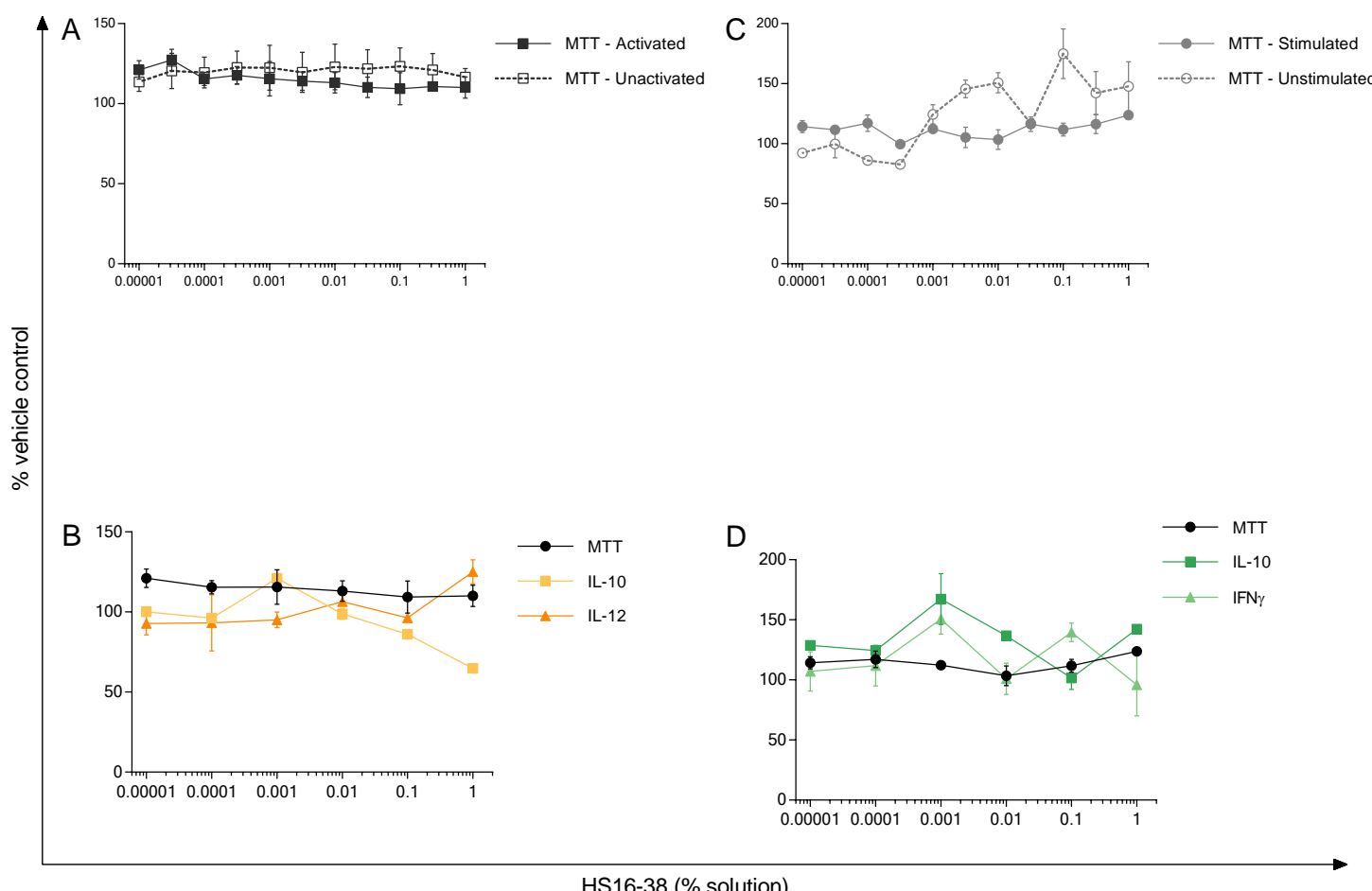

HS16-38 (\% solution)

Figure 9.38. Concentration identification for HS16-38 using stimulated macrophages and Th cells Activated macrophages were treated with HS16-38 and viability (A) and IL-10 and IL-12 (B) was assessed. Similarly, Helper T cells were activated in the presence of HS16-38to generate MTT (C) and IL-10 and IFN $\gamma$ data (D). Data were used to select concentrations for further testing as described in (Ch 3, section 5.3.1)

HS16-38 was non-toxic to macrophages and splenocytes. Three high concentrations for macrophages (0.01, 0.1 and $1 \%$ ) covered the changes to cytokine production that was observed. Similarly, the four highest concentrations (0.001, 0.01, 0.1 and 1\%) of HS16-38 covered the fluctuating changes to cytokine production seen from stimulated Th cells. 


\subsubsection{Screening data for HS16-38}

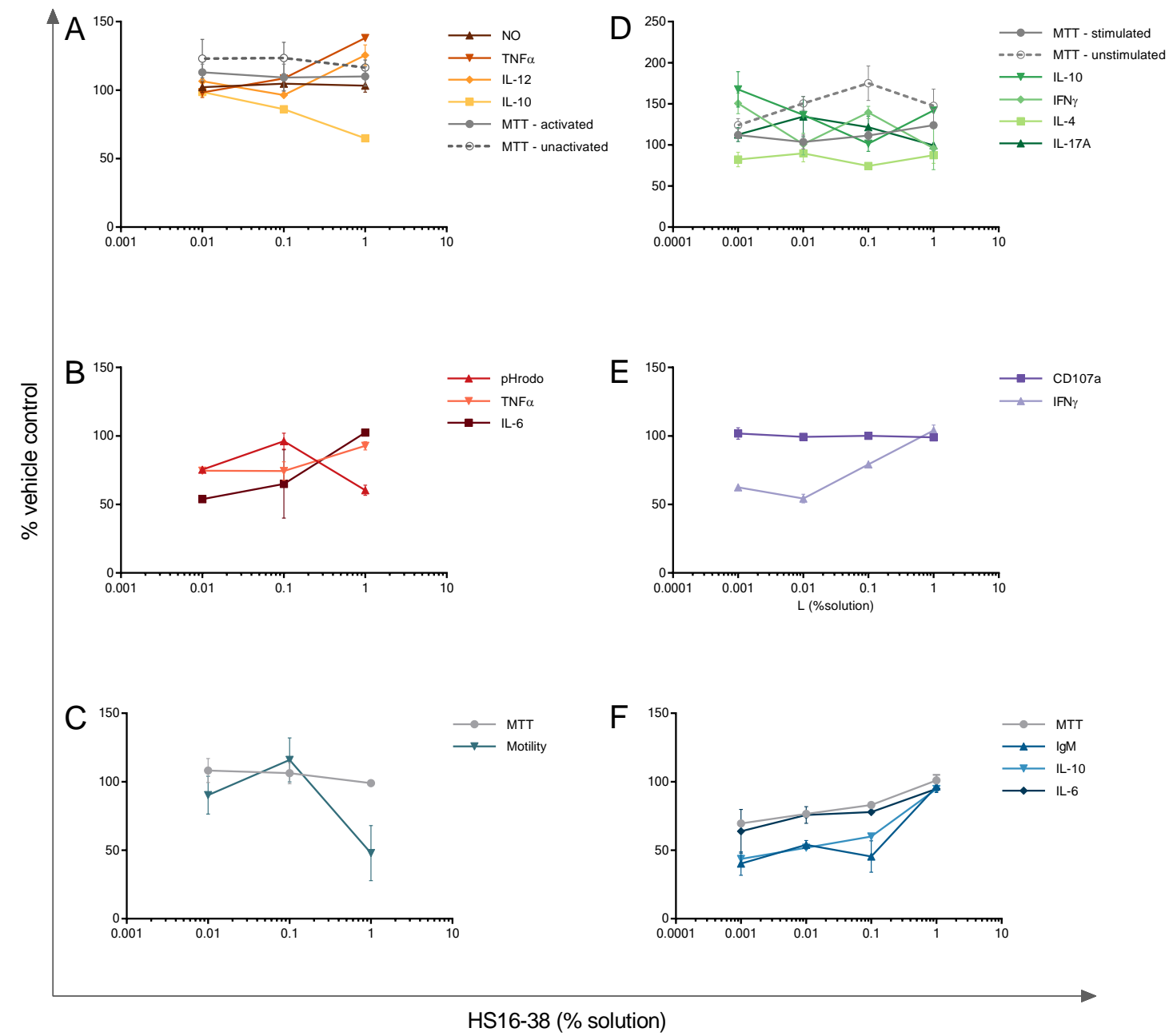

Figure 9.39. Screen outputs for HS16-38

HS16-38, at indicated concentrations, was run through the six assays. Macrophage activation (A), phagocytosis (B) and motility (C) are macrophage-based assays. In the right column are the splenocyte-based assays: helper T cell activation (D), CTL degranulation (E) and B cell activity (F). Experiments were conducted as explained in thesis methods (Ch 2). Shown are compound-induced changes compared to vehicle controls (i.e. \% vehicle). Closure in vehicle-treated wells during the motility assay averaged $74.0 \%$ (SD = 15.9\%). Data are means and SEM from each experiment which was conducted once in duplicate. 


\subsubsection{NTR1}

\subsubsection{Concentration selection for NTR1}

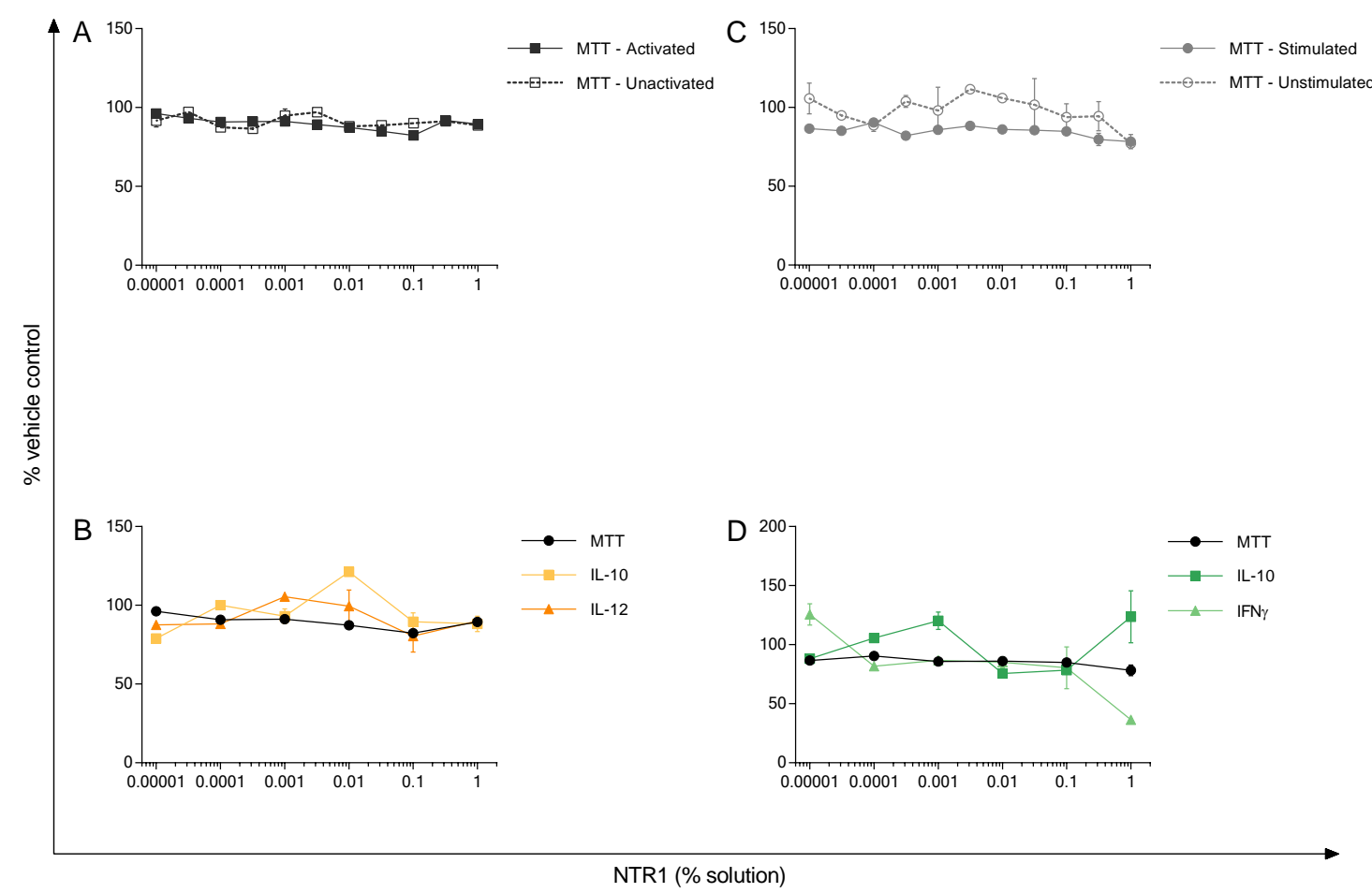

Figure 9.40. Concentration identification for NTR1 using stimulated macrophages and Th cells Activated macrophages were treated with NTR1 and viability (A) and IL-10 and IL-12 (B) was assessed. Similarly, Helper T cells were activated in the presence of NTR1 to generate MTT (C) and IL-10 and IFN $\gamma$ data (D). Data were used to select concentrations for further testing as described in (Ch 3, section 5.3.1)

NTR1 was not toxic to cells. For macrophages, the high $0.01,0.1$ and $1 \%$ solutions were selected. For splenocytes, the high concentrations of $0.001,0.01,0.1$ and $1 \%$ were selected. These concentrations saw changes to cytokine production by activated Th cells. The high concentration, specifically, caused an increase in IL-10 and a decrease in IFN $\gamma$ production. 


\subsubsection{Screening data for NTR1}

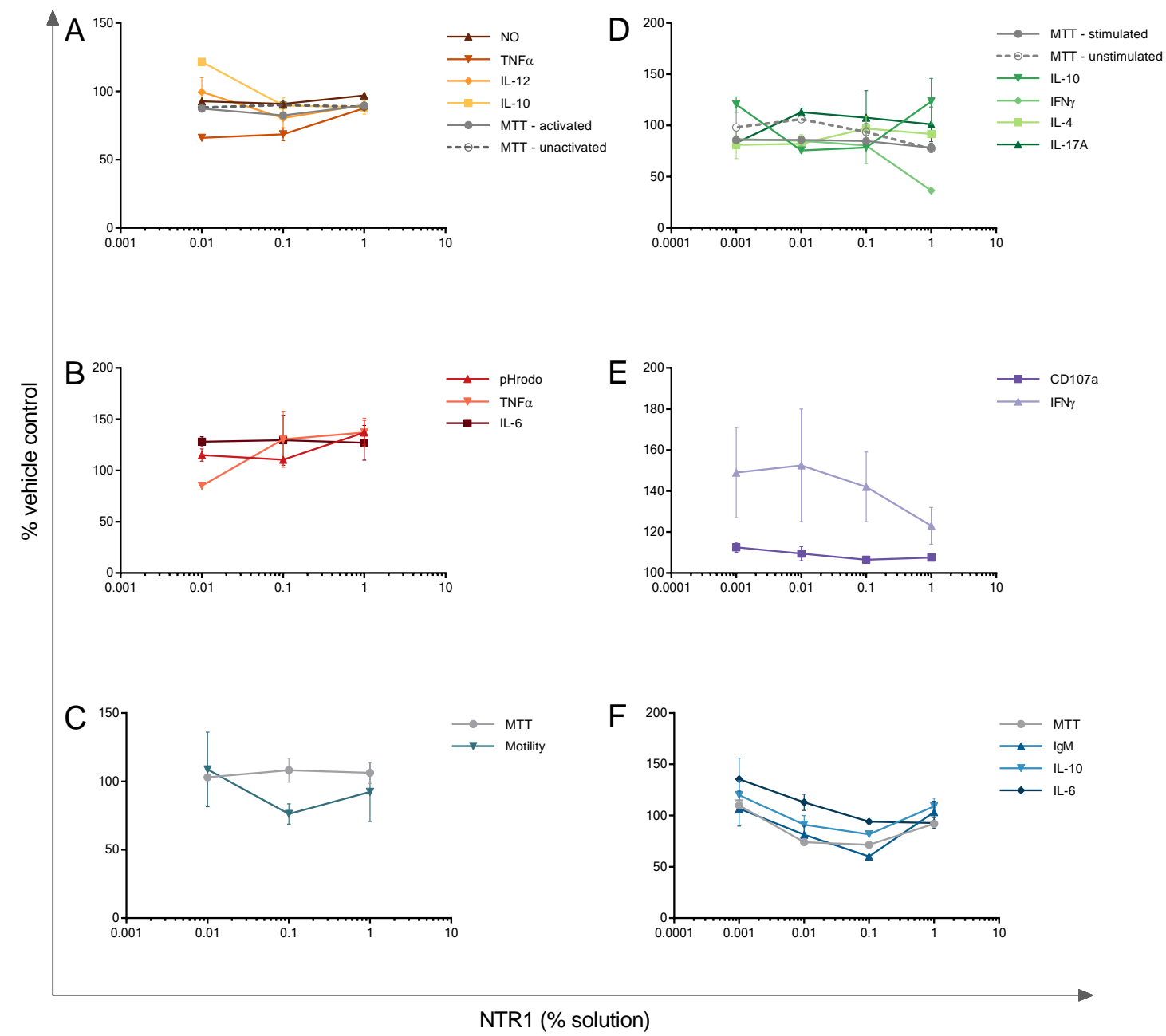

Figure 9.41. Screen outputs for NTR1

NTR1, at indicated concentrations were run through the six assays. Macrophage activation (A), phagocytosis (B) and motility (C) are macrophage-based assays. In the right column are the splenocyte-based assays: helper T cell activation (D), CTL degranulation (E) and B cell activity (F). Experiments were conducted as explained in thesis methods (Ch 2). Shown are compound-induced changes compared to vehicle controls (i.e. \% vehicle). Closure in vehicle-treated wells during the motility assay averaged $74.0 \%(S D=15.9 \%)$. Data are means and SEM from each experiment which was conducted once in duplicate. 


\subsubsection{NTR2}

\subsubsection{Concentration selection for NTR2}

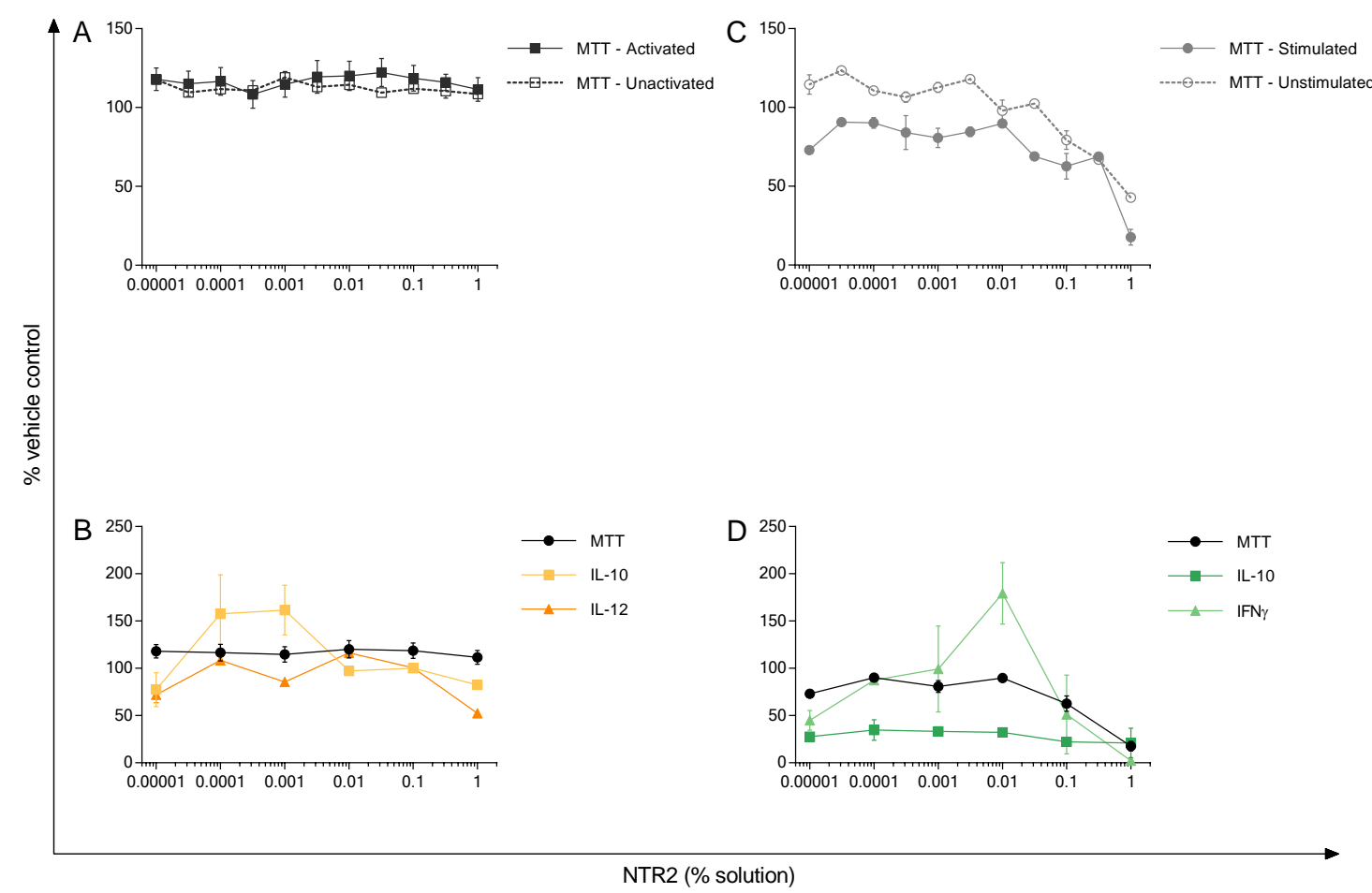

Figure 9.42. Concentration identification for NTR2 using stimulated macrophages and Th cells Activated macrophages were treated with NTR2 and viability (A) and IL-10 and IL-12 (B) was assessed. Similarly, Helper T cells were activated in the presence of NTR1to generate MTT (C) and IL-10 and IFN $\gamma$ data (D). Data were used to select concentrations for further testing as described in (Ch 3, section 5.3.1)

NTR2 was not toxic to macrophages, so four high concentrations were selected $(0.001$, 0.01, 0.1 and 1\%). For splenocytes, the four highest non-toxic concentrations were chosen for testing for NTR2. These included $0.0001,0.001,0.01$ and $0.1 \%$ solutions. These concentrations also decreased IL-10 production, and covered the spike in IFN $\gamma$ production seen with NTR2. 


\subsubsection{Screening data for NTR2}

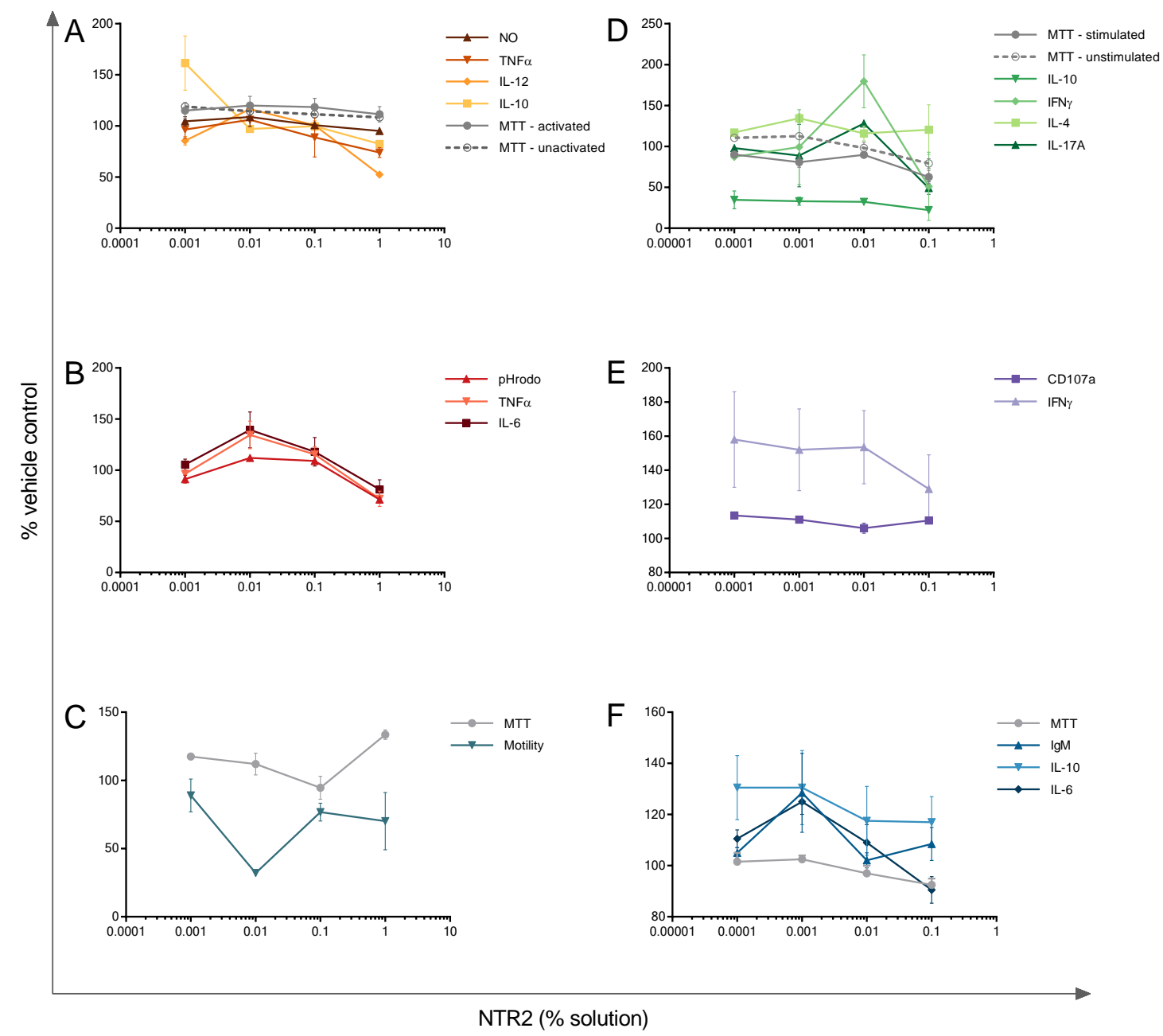

Figure 9.43. Screen outputs for NTR2

NTR2, at indicated concentrations were run through the six assays. Macrophage activation (A), phagocytosis (B) and motility (C) are macrophage-based assays. In the right column are the splenocyte-based assays: helper T cell activation (D), CTL degranulation (E) and B cell activity (F). Experiments were conducted as explained in thesis methods (Ch 2). Shown are compound-induced changes compared to vehicle controls (i.e. \% vehicle). Closure in vehicle-treated wells during the motility assay averaged $74.0 \%(S D=15.9 \%)$. Data are means and SEM from each experiment which was conducted once in duplicate. 


\subsubsection{NTR3}

\subsubsection{Concentration selection for NTR3}

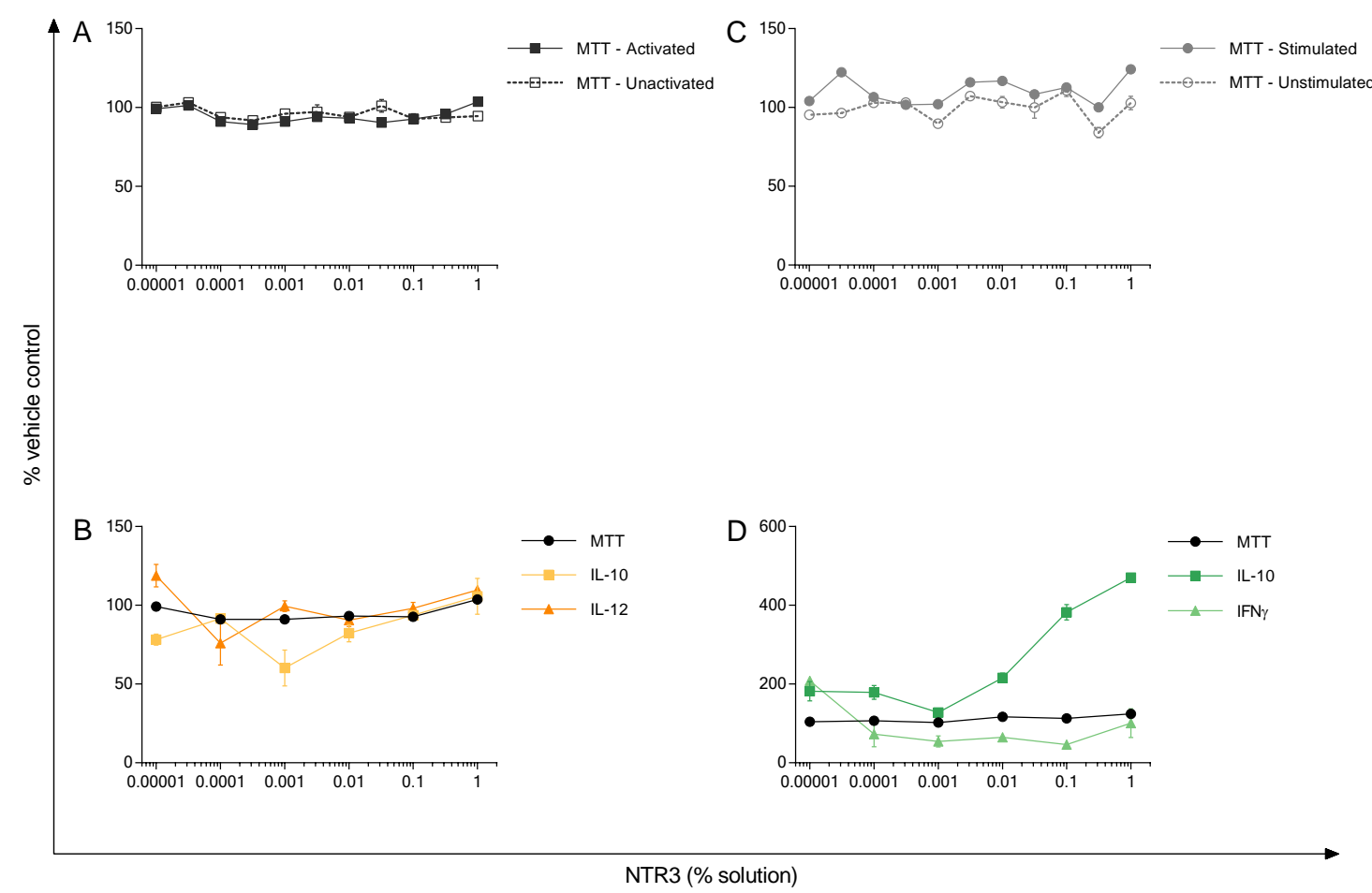

Figure 9.44. Concentration identification for NTR3 using stimulated macrophages and Th cells Activated macrophages were treated with NTR3 and viability (A) and IL-10 and IL-12 (B) was assessed. Similarly, Helper T cells were activated in the presence of NTR3 to generate MTT (C) and IL-10 and IFN $\gamma$ data (D). Data were used to select concentrations for further testing as described in (Ch 3, section 5.3.1)

NTR3 was also non-toxic to macrophages and splenocytes. For both cell types, four high concentrations were selected $(0.001,0.01,0.1$ and $1 \%)$. For both cell types these concentrations covered the interesting effects NTR3 had on cytokine production, including the increased IL-10 production and decreased IFN $\gamma$ production from Th cells. 


\subsubsection{Screening data for NTR3}

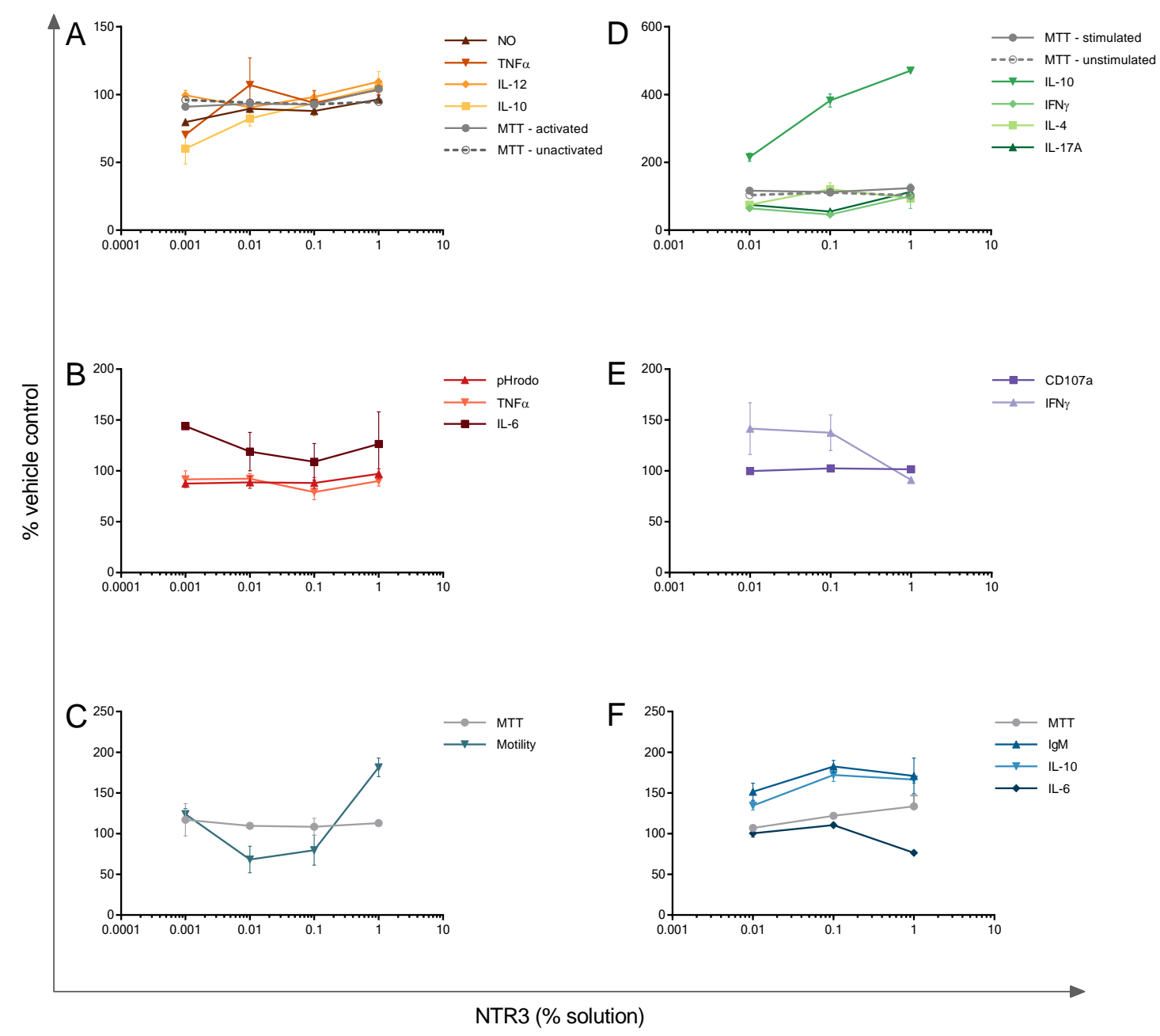

Figure 9.45. Screen outputs for NTR3

NTR3, at indicated concentrations were run through the six assays. Macrophage activation (A), phagocytosis (B) and motility (C) are macrophage-based assays. In the right column are the splenocyte-based assays: helper T cell activation (D), CTL degranulation (E) and B cell activity (F). Experiments were conducted as explained in thesis methods (Ch 2). Shown are compound-induced changes compared to vehicle controls (i.e. \% vehicle). Closure in vehicle-treated wells during the motility assay averaged $74.0 \%(S D=15.9 \%)$. Data are means and SEM from each experiment which was conducted once in duplicate. 